\title{
ON CHIP COMPLEX BREAST TUMOUR MICROENVIRONMENT: APPLICATION TO RESEARCH IN NANOMEDICINE
}

Jean-Baptiste Blondé

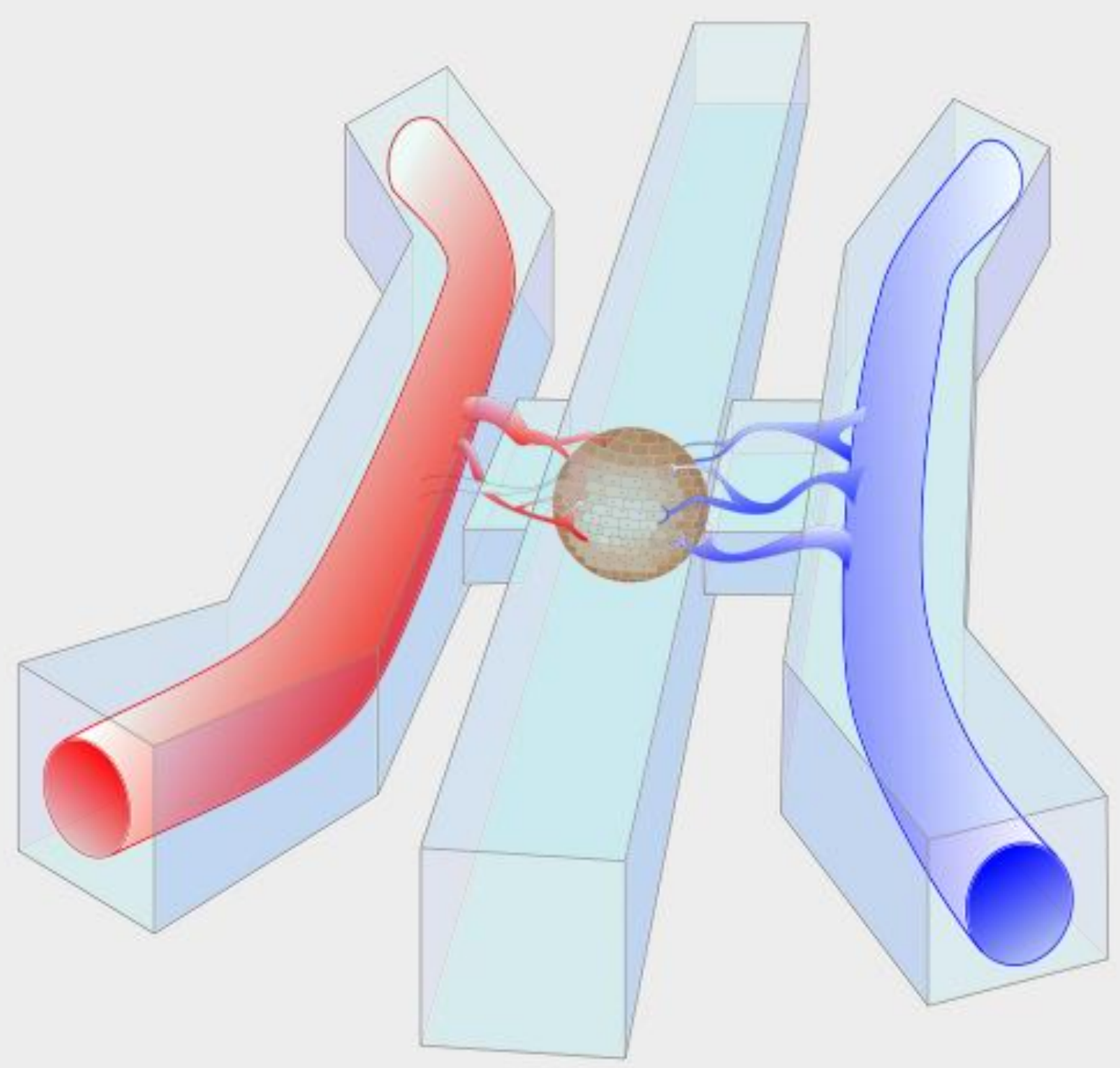



ON CHIP COMPLEX BREAST TUMOUR

MICROENVIRONMENT: APPLICATION TO RESEARCH IN NANOMEDICINE

Jean-Baptiste Blondé 
This dissertation has been approved by:

supervisor

Prof. dr. S. Le Gac

Cover design: Jean-Baptiste Blondé

Printed by: IDENTIC Impresssion, Cesson-Sévigné, France

ISBN: 978-90-365-5059-8

DOI: $10.3990 / 1.9789036550598$

URL : https://doi.org/10.3990/1.9789036550598

(C) 2020 Jean-Baptiste Blondé, The Netherlands. All rights reserved. No parts of this thesis may be reproduced, stored in a retrieval system or transmitted in any form or by any means without permission of the author. Alle rechten voorbehouden. Niets uit deze uitgave mag worden vermenigvuldigd, in enige vorm of op enige wijze, zonder voorafgaande schriftelijke toestemming van de auteur. 


\section{Doctorate board:}

Chairman / secretary:

supervisor:

Committee Members: prof.dr. J.N. Kok

prof.dr.ir. S. Le Gac

prof.dr. L.W.M.M. Terstappen

prof.dr. J. Prakash

dr. I. Lentacker

prof. dr. C. Tokarski

prof.dr.ir. S. le Gac

prof. dr. Z. Brzózka 


\title{
ON CHIP COMPLEX BREAST TUMOUR MICROENVIRONMENT: APPLICATION TO RESEARCH IN NANOMEDICINE
}

\author{
DISSERTATION
}

to obtain

the degree of doctor at the Universiteit Twente,

on the authority of the rector magnificus,

Prof.dr. T.T.M. Palstra,

on account of the decision of the doctorate board

to be publicly defended

on Friday 18 September 2020 at 12.45 uur

by

Jean-Baptiste Blondé

Born December $20^{\text {th }} 1990$ in Rennes, France 


\section{TABLE OF CONTENTS}

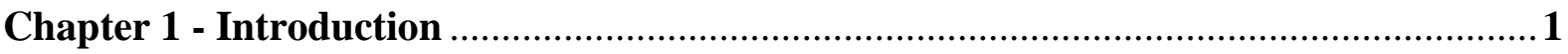

Chapter 2 - Multicellular tumour spheroid proteomic analysis: how size influences protein expression on breast cancer cells. .25

Chapter 3 - Studying the Cellular and Molecular Organization in Multicellular Tumour Spheroids using CLARITY

Chapter 4 - Molecular and Cellular organization of the 3D Tumour Spheroid .91

Chapter 5 - Tumour-on-a-Chip Platform to Evaluate Nanomedicine Penetration in Coculture Tumour Spheroids

Chapter 6 - Toward Vascularized Breast Cancer on Chip: Approaching the Complex Tumour Micro Environment 153

Chapter 7 - Summary and Outlook

Samenvatting.

About the author

Scientific Output 190

Acknowledgment 192 
Chapter 1 - Introduction 


\section{CANCER DISEASE AND TUMOUR MICRO-ENVIRONMENT}

\subsection{DEFINITION AND STATISTICS}

Cancer is one of the most threatening diseases notably in developed countries (Figure 1-1.a), with 18 million new cases reported worldwide in 2018 and a mortality rate of 9.5 million deaths worldwide the same year [1]. Due to the many different types of cancer, affecting virtually any organ, the term "cancer" has been generalized to define the uncontrolled growth of cells, forming masses, or tumours, causing compressions or congestions in the organ they grow in. Moreover, if the cancer progresses into later stages, in most cases, cancer cells dissociate from the original tumour site, to escape into the blood stream. The resulting so-called circulating tumour cells (CTCs) can give rise to the formation of secondary tumours in other organs, in a process called metastasis [3].

The disease is initiated by the alteration of certain genes (e.g., through single-nucleotide polymorphism), named proto-oncogenes [4], involved in cell growth and proliferation. These genes mutate into oncogenes, which can prevent the cell programmed death, known as apoptosis, and up-regulate cell proliferation. The risk for an individual of contracting cancer is depending on the number of copies of the same proto-oncogene he/she possesses. As a result, people with ancestors that have suffered from cancer have a higher predisposition of contracting it [5]. Although spontaneous proto-oncogene mutation is possible, it is most often triggered by environmental factors [6]. Several lifestyle factors (e.g., tobacco, obesity), and repeated exposure to pollutants (e.g., chemicals, radiation) have been identified to trigger mutations, but the exact aetiology of the pro-oncogene activation has not yet been fully elucidated [7].

Cancer is divided in different categories, depending on the cells and/or organs it affects (e.g., leukaemia, carcinoma, lymphoma, or sarcoma), each presenting sub-types (e.g., invasive, benign, malignant, or hormonal), leading to a wide variety of diseases, which can therefore not be cured with a single treatment [8]. In this thesis, we focus on the most affected organ by cancer for women worldwide: breast cancer (Figure 1-1.b). More specifically, we investigate its most common type, the invasive ductal carcinoma ( $80 \%$ of all breast cancer), which affects two thirds of women above the age of 55 [1]. A "carcinoma" refers to a cancer, which develops in an epithelial tissue (e.g., skin or organ lining tissue). "Ductal" specifies that the cancer 
Tumour progression is heavily influenced by the stroma, which is the supportive framework of an organ, and which is composed of connective tissues and blood vessels (Figure 1-2). The cells in the connective tissue include fibroblasts, adipocytes, macrophages and leukocytes. Among these, fibroblasts, which are the most abundant [11-13], are responsible for the production of Extra Cellular Matrix (ECM), and play an essential role in wound healing. In the presence of cancer cells, however, fibroblasts become irreversibly "activated" by cancer cell-secreted growth factors. These activated fibroblasts, also known as cancer-associated fibroblasts (CAFs), play a crucial role in sustaining the tumour by producing abundant amounts of ECM, leading to the formation of a higher density tissue [14, 15]. CAFs also secrete growth factors (e.g., fibroblast growth factor (FGF)) promoting the tumour growth [15], chemokines stimulating the recruitment of other stromal cells, and in some cases pro-inflammatory factors contributing to the recruitment of immune cells [16]. Multiple studies have demonstrated the importance of CAFs in the progression of invasive ductal carcinoma [17-19].

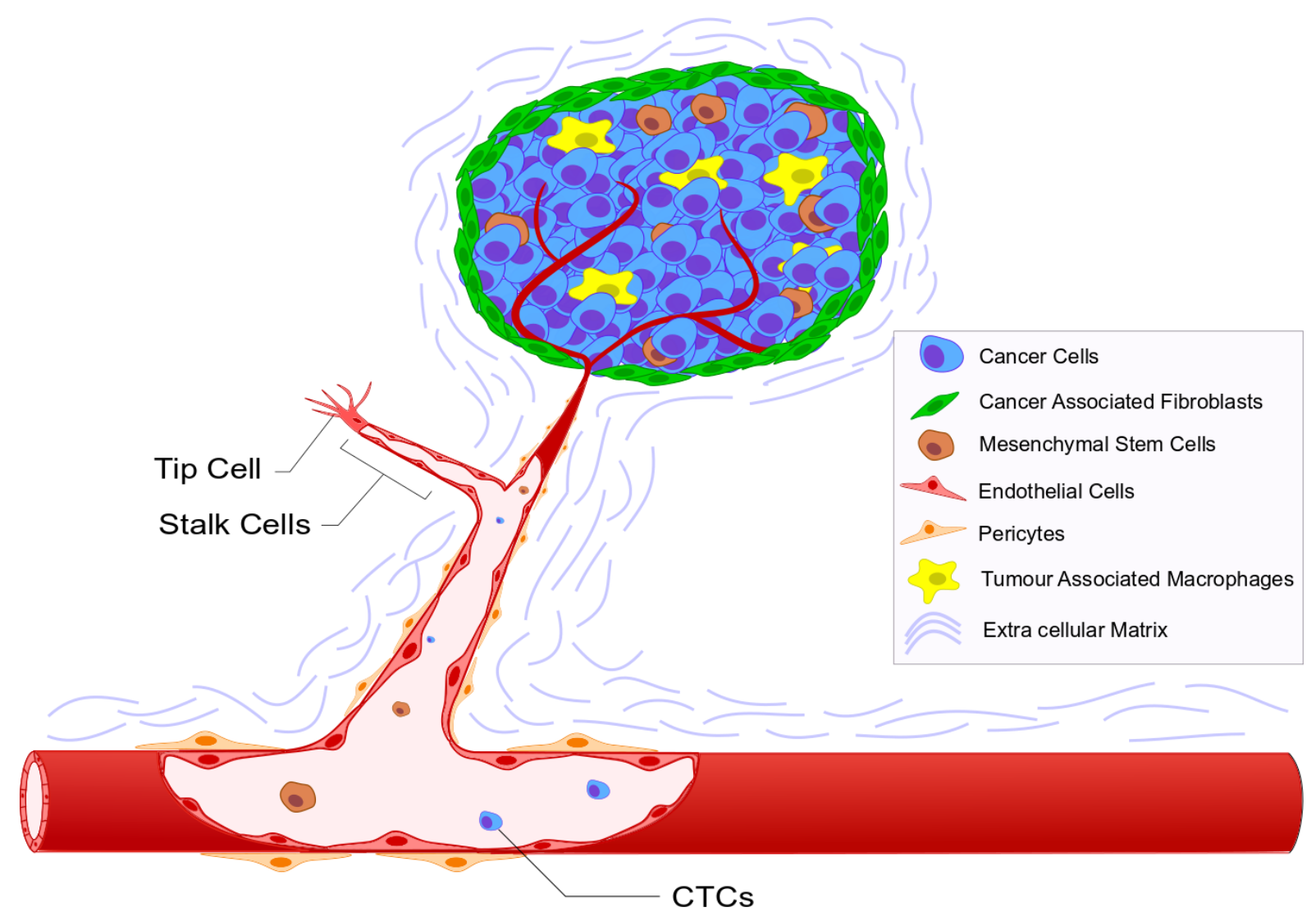

Figure 1-2 The Tumour Microenvironment (TME), a complex structure supported by stroma and vascularization. The tumour secretes growth factors, triggering sprouting of blood vessels via angiogenesis and recruiting cells (e.g., Mesenchymal Stem Cells), to further proliferate. 
As a tumour proliferates rapidly, its needs for nutrients and oxygen are relatively high. This rapid growth and the abnormal density of the tissue leads to the creation of gradients of oxygen and nutrients toward the core of the tumours, resulting in the existence of different cell phenotypes depending on their specific position in the tumour tissue: cells present in close proximity to blood vessels are highly proliferating, but as their distance to the blood vessels increases, they become quiescent (non-proliferative), or even sometimes necrotic [20, 21]. To prevent the formation of hypoxic or necrotic zones, a tumour recruits endothelial cells towards the hypoxic regions, through a process called angiogenesis, by secreting growth factors, such as vascular endothelial growth factors (VEGF) and FGF. In response, the already existing blood vessels generate a network of capillary vessels, or neovasculature, in the tumour, to supply the tumour with the required nutrients and oxygen [22-25]. These new blood vessels, which would normally be surrounded by smooth muscle cells and pericytes for their support and stabilization, have been found to be leakier than in healthy tissues, due to their accelerated growth, which, as a result, can facilitate the extravasation of the tumour cells into the blood stream $[26,27]$.

Other cells of the connective tissue are found in the TME. Lymphocytes are often abundant ( $\sim 10 \%$ of total mass); they promote tumour progression and tumour metastasis [28, 29]. Tumour-associated macrophages (TAMs), which are highly present and mainly in the hypoxic or necrotic regions of the tumour, are also responsible for the production of angiogenic factors [30, 31]. Finally, other types of stromal cells participate in the TME stability: for instance, mesenchymal stem cells (highly present in breast cancer) can be recruited from the bone marrow and differentiate into CAFs, smooth muscle cells or pericytes inside the tumour $[32,33]$.

\subsection{A DISEASE RESISTANT TO TREATMENTS}

A unique property of cancer cells, unlike external pathogens (e.g., virus, bacteria) is their similarity with healthy cells. As a result, it is most difficult to establish a treatment that can specifically target the diseased tissue without affecting the healthy cells. Therefore, the two main currently established treatments focus on the major difference between cancer cells and healthy cells, which is their abnormal growth rate.

The first treatment approach is radioactive therapy or radiotherapy which includes two predominant variants: teletherapy, by which ionizing radiations are directed toward the tumour 
site with an external beam ( $c a .44$ cycles over two months); and brachytherapy, by which a radioactive seed is implanted at the target area. These radiations damage double stranded DNA in the exposed cells. Studies have indicated that in general, cancer cells had much more difficulty to recover from radiation exposure [34]. Radiotherapy has proven to be relatively successful: in prostate cancer (acinar adenocarcinoma) up to $95 \%$ of the tumours are eradicated using external-beam radiation therapy, with a 98.8\% 5-year survival rate [35]. A major limitation of this method is the presence of a gradient of oxygen inside the tumour, since in a hypoxic region cells are 2-3 times more resistant to radiation exposure [36]. Some studies are now focusing on integrating systems or compounds to overcome this hypoxia related resistance, and developing combined therapeutic approaches, to improve the overall outcome of the treatment [37]. Additionally, radiotherapy is very expensive, time-consuming, and limited to tumour accessible to X-ray imaging. Prolonged radiation exposure can cause complications in the exposed areas and surrounding tissues. The most common reported longterm side effects are swelling and stiffening of the exposed tissues, and infertility if reproductive organs are exposed to radiation.

The second approach, chemical therapy or chemotherapy, targets fast-proliferative cells, like the cancer cells, and interrupts their cell-division cycle. A wide-variety of such drugs exist, and they can be classified into different categories. Alkylating antineoplastic agents were the first chemotherapeutic drugs, originally used by the German empire in "mustard gas" during World War I [38]. They bind to DNA strands in two different locations to cause them to break when a cell divides or attempts to repair itself [39]. Since then, other types of chemotherapeutic agents have been developed, targeting other components in the cells; by for instance stopping the topoisomerase enzyme activity (topoisomerase inhibitors) involved in the DNA structural change during replication or transcription, or the cell cycle (cytotoxic antibiotics, such as doxorubicin) [40]. The main challenge of this approach is the very small amount of active ingredients being retained at the target site after their injection, and its low absorption by the targeted cells. The main reason for this limited uptake is the lack of specificity of the drugs. The largest part of the chemotherapeutic agents is either metabolized in the liver or filtered by the kidneys. Therefore, dosages have to be limited to prevent detrimental sideeffects to otherwise healthy organs.

Since these two approaches specifically focus on the fast-proliferative cells which are found on the outside of the tumours, next to the blood vessels, the main noticeable effect after treatment, is a shrinkage of the tissues. If successful, the tumour shrinks until it is completely 
eradicated. In many cases however, this treatment is insufficient and tumours only partially decrease in size. The last option is then to remove the tumour physically, through surgery. Because of the risk imbued with open surgery (e.g., other tissue damage, risk for infections) this option is only chosen last, and in combination with one of the other treatments. Surgery is also not applicable in case the tumour cannot be reached, especially in the brain or spine, where the risks of compromising vital functions (e.g., brain functions, immune system) are extremely high.

For breast cancer, which is the focus of this thesis, the currently most recommended approach is surgery followed by radiotherapy, to destroy any cancer cells that may be left behind. In cases where the tumour is large, chemotherapy can be used to shrink its size. There exist two different types of breast surgery, lumpectomy where only the tumour and its surrounding tissue are removed, and mastectomy where the entire breast is ablated. If lumpectomy is performed, it is followed by radiotherapy. The success rate of this method is very high, even for advanced tumour, but despite estimated not to be life threatening, it can lead to life complications, as well as emotional trauma [41, 42]. Additionally, cancer treatment is sometimes followed by hormone therapy (e.g., Tamoxifen) lowering the production of oestrogen, to decrease the risk of cancer resurgence (40-50\%) [43]. However, recent findings have indicated that hormonal treatment can lead to cognitive impairment [44]. Therefore, significant work is devoted to the development of new chemotherapeutic drugs, to achieve similar success rate, while limiting the need for surgery, as well as the aforementioned side effects.

\subsection{TOWARD NANOTECHNOLOGY-BASED SMART TREATMENTS}

\subsubsection{Nanoparticles as drug delivery systems}

More recently, new and promising approaches have emerged, used for both diagnosis and drug delivery, which are called nanomedicines. These nanomedicines can be prepared from a variety of organic and inorganic materials, and used to either attached the drug to them, or to encapsulate it [45]. In cancer, the drug-conjugated nanomedicines (e.g., antibody-drug, polymer-drug) are currently the most established nanomedicine-based therapeutics, thanks to their small size $(5-20 \mathrm{~nm})$ facilitating their penetration and uptake in the tumour. An approved drug conjugate by regulatory authorities is the antibody-drug conjugate trastuzumabemtansine, targeting the protein HER-2, which is overexpressed in some breast tumours, and which has shown great success in slowing down the tumour progression [46]. Another type of 
drug conjugates currently in development consists of radiopeptides, which are drug conjugated to an isotope (e.g., Tc-99 or Y-90) through a peptide linker, exploiting the previously mentioned radio sensitivity of cancer cells $[47,48]$. While these drug conjugate have shown promises, they are currently limited by their carrying capacity, the complexity of the coupling process, and the low level of target specificity.

a)

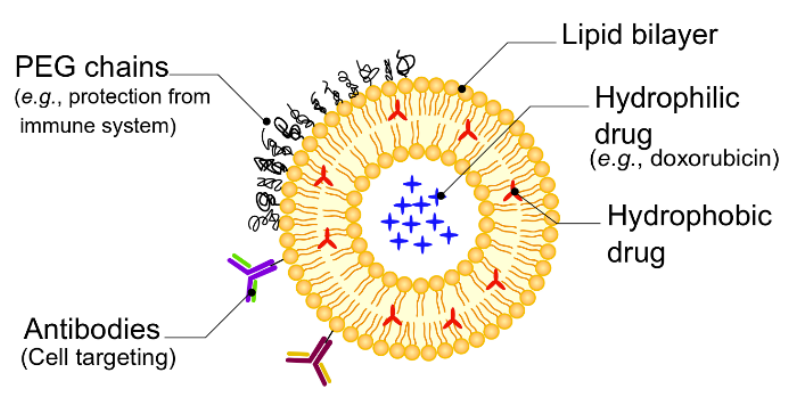

b)

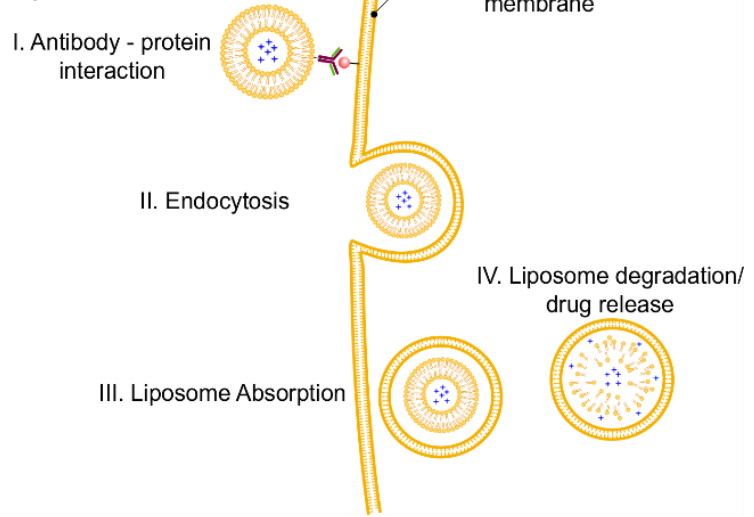

Figure 1-3 Liposome: a versatile example of nanomedicines. a) Schematic representation of a liposome ( 100 $\mathrm{nm}$ in size), decorated with added features attached to its lipidic shell (e.g., PEG chains, antibodies), to increase the uptake of the targeted drug while limiting its toxicity. b) Endocytosis process, orchestrated by a targeting antibody present on the liposome and a cell membrane protein.

The second category of nanomedicines comprises the encapsulating nanocarriers. These nanocarriers are larger in size $(30-200 \mathrm{~nm})$, and can encapsulate a greater amount of therapeutic agents. As a result, it is estimated that, up to four times more therapeutic agents can be administered using nanocarriers instead of free drugs, while limiting drug-induced toxicity. These nanocarriers can be divided into two categories based on the materials they consist of: organic and inorganic carriers. The most common organic nanocarriers are lipid-based, especially micelles and liposomes, which can both transport hydrophobic and hydrophilic therapeutic agents (Figure 1-3.a). For certain cancer types (e.g., breast cancer, lymphoma), some lipid-based nanomedicines are already on the market. For instance, DOXIL ${ }^{\circledR}$ is a liposome encapsulating doxorubicin. Other organic nanocarriers include natural or synthetic polymers (e.g., dendrons) [49]. The first established polymeric nanoparticle, BIND-014, which is currently in Phase 2 trial, is a poly(lactic-co-glycolic acid) (PLGA) and polyethylene glycol (PEG) conjugate [50]. This nanomedicine can deliver ten times more of the therapeutic agent docetaxel to targeted prostate cancers, compared to the freely injected drug. Finally, nanocarriers can also be prepared from inorganic materials (e.g., silica, gold, quantum dots crystals) [51]. These nanoparticles are mostly used for diagnostic purposes, by taking 
advantage of some unique properties of the material. Currently there are no inorganic nanomedicine available on the market, but some of them have reached the first stage of human trials, such as CYT-6091, a gold nanoparticle coated with PEG onto which Tumour Necrosis Factor alpha (TNF- $\alpha$ ) is covalently attached, which is currently tested against melanoma and sarcoma cancers [52].

\subsubsection{Targeting methods to improve efficacy}

Since the nanocarriers are much larger than the soluble drugs, it can become challenging for them to reach the area of interest; Therefore, targeting approaches have been developed. The first type of targeting is passive and relies on the leakiness of the blood vessels neighbouring the tumour as previously discussed. This passive approach has however proven to be insufficient. The second and most established approach is the active targeting, where the nanomedicine is covalently attached to specific antibodies, which can recognize the cells of interest by interacting with specific membrane receptor proteins. The nanomedicines can then accumulate at the target site, and increase their uptake through processes like endocytosis, where they are "ingested" by the cell and the therapeutic agents are released (Figure 1-3.b). Additionally, the nanomedicines are often coated with PEG, a hydrophilic polyether which has demonstrated to protect the nanomedicines from fouling, the accumulation of particles present in the blood on the surface. It also helps protect the nanomedicines by hiding them the from immune system [53].

Another method of active targeting consists of locally releasing the therapeutic agents from the nanocarriers, which can be achieved using either internal (e.g., $\mathrm{pH}$ change, like in cancer tissues as a result of anaerobic glycolysis) [54], or external actuation (e.g., laser, ultrasound) [55]. Thereby, higher concentrations of free drugs can be released locally in the tissue directly while limiting the toxicity.

Studies are no longer limited to targeting the cancer cells, but also other actors of the TME involved in the tumour progression, such as the CAFs, TAMs, or the tumour-induced neovasculature [56-58]. Nanomedicines may represent the next generation of drug treatment, but they first need to be tested in laboratory for validation before going on to animal and human trials. However, due to the structural and functional complexity of these nanomedicines, current FDA approved laboratory models, known as 2D in vitro models, are insufficient to test their full potential (e.g., transport and targeting). 


\section{THE IN VITRO MODEL, THE FIRST PATIENT FOR THE (NANO)MEDICINES}

\subsection{ON THE IMPORTANCE OF IN VITRO MODELS}

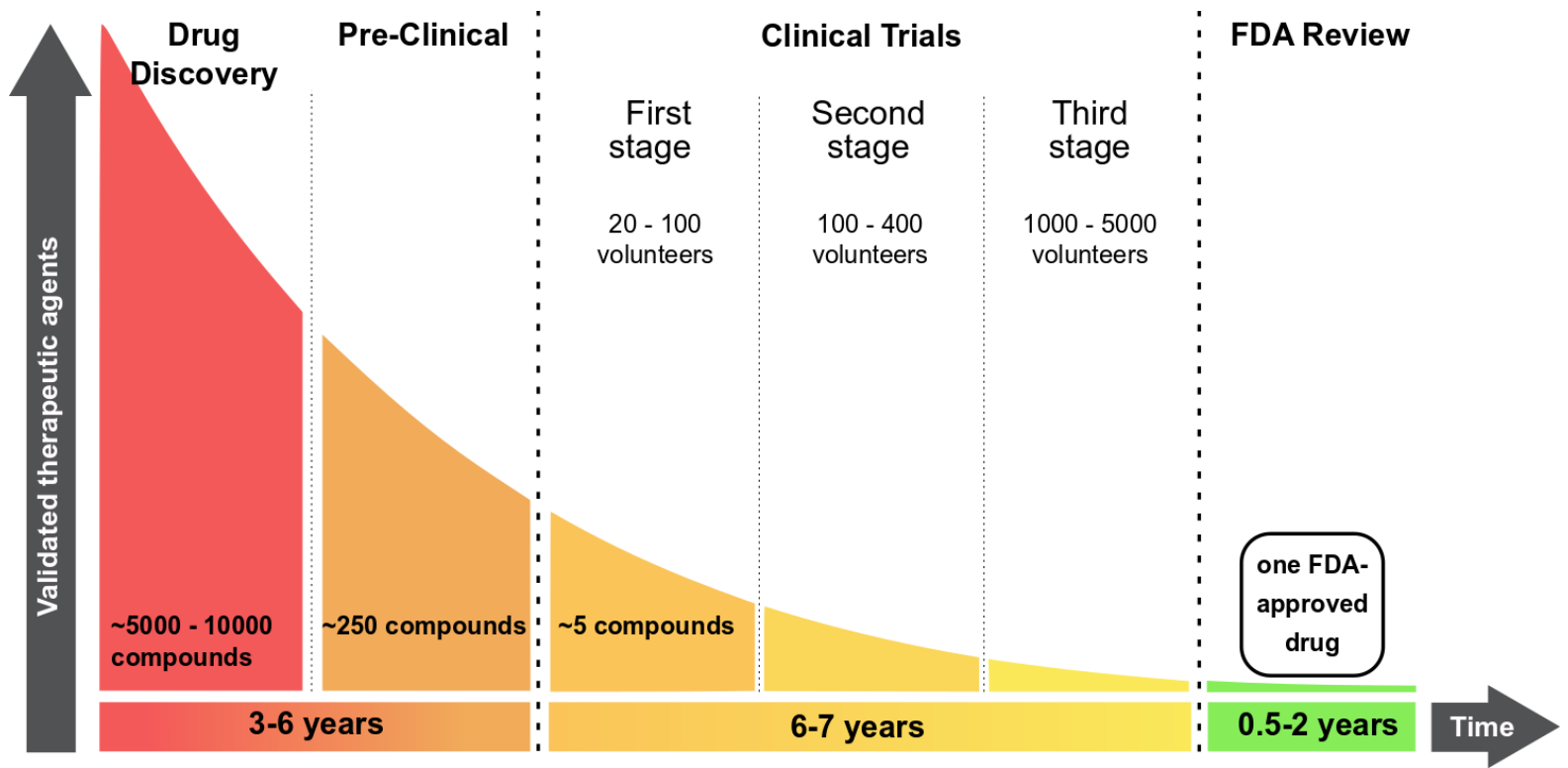

Figure 1-4 Graph of a therapeutic agent development timeline. From 5000-10000 compounds initially synthesized against a molecular target and tested in vitro, only 250 reach the pre-clinical trials (on animals), and only 5 the clinical trials (on humans). On average, out of the 5000-10000 initial compounds, only 1 goes successfully through all the different stages, to maybe get a FDA approval, after a minimum of 10-15 years of research [2].

Before they can be approved (e.g., by the Food and Drug Administration (FDA, USA) or the European Medicine Agency (EMA, Europe)) and become available on the market, nanomedicines, like any other new drugs, must go through different steps of development and evaluation as illustrated on Figure 1-4. The process starts in the lab with in vitro models, which are usually 2D cell cultures (or monolayer) or tissues (see below), followed by animal testing, and finally by different stages of human clinical trials [59]. Each year, hundreds of new drugs are developed, but very few actually reach the market, which can be due to their toxicity (sideeffects), or simply their overall inefficacy. Too often however, such negative results are noticed at a late stage of the animal testing or even human clinical trials, after years of development and considerable amounts of money have been spent $[2,60]$. 
One of the identified factors responsible for this failure is the lack of adequate models to test the drugs, which do not directly translate to the human physiology. Indeed, the most widely established in vitro model consists of a 2D monolayer of cells, extracted from a donor, and grown on a flat and rigid plastic dishware. The cells are even sometimes genetically modified to become immortalized. These cells, also known as cell lines, are commercially available, and present significant differences with cells found in the human body. Furthermore, these 2D monolayers are also mostly limited to one single cell type (e.g., cancer cell line), whose morphology and physiology do not emulate the in vivo conditions. Moreover, these simplistic in vitro models contain none of the previously mentioned features, such as gradients of oxygen and nutrients, vascular networks, or an immune system. As a consequence, their phenotype and gene and protein expression profiles, as well as their response to drugs, do not correspond to those found in the human body (see chapter 2)[61].

Using in vitro models of the in vivo human situation is essential in drug evaluation process, as they are the simplest to establish, they allow for a fast parallelisation of experimental conditions, and they can very rapidly provide essential information like the efficacy or toxicity of drugs $[62,63]$. Because of the limitations presented by these models, drugs are next tested on animals, usually rodents, who can provide the missing physiological and systemic features. However, the animal physiology does not compare to the human one. Additionally, due to ethical concerns, animal testing is frowned upon and a series of guidelines known as the three R's (Replacement, Reduction, Refinement) was proposed in 1959 by W. M. S. Russell and R. L. Burch, to improve animal welfare and encourage the pursuit of alternative approaches of drug testing. As a result, In the last years, efforts have been made to develop more accurate and physiologically relevant in vitro models by artificially recreating some of these missing features without the need for live animals [61].

\subsection{FROM 2D TO 3D IN VITRO MODELS}

Recreating organs in the lab is one of the major current challenges of notably the field of tissue engineering, due to their structural complexity, and the amount of different cell types involved. At the micrometre scale however, 3D in vitro models can be developed, which can still provide valuable information. They differ from standard 2D models by the complexity they can offer; combining multiple cell types, organized structure, and most important, a 3D morphology of the cells. Two main techniques have been developed to produce "simple" yet 
faithful 3D in vitro models. The first approach uses solid scaffold from biocompatible materials (e.g., hydrogels) in which cells grow following the scaffold's shape [64]. The second category relies on the spontaneous aggregation of cells by eliminating their interaction with the substrate they are on, either by encapsulating them (e.g., flow focusing) [65, 66] or using ultra-low attachment plates [67], as reviewed by S. Sant and P.A Johnston [68]. The ultra-low attachment plates method especially allows generating high throughputs of spheroids, with simple protocols, through three main different techniques: the well array, the hanging drop, and spinning disk. First, the spinner flask method continuously stirs cells in suspension, preventing them from aggregating to any surface, favouring their aggregation but limiting control over the size of the spheroids [69]. The well array approach introduces the cells in arrays of microwells whose surface properties have been modified to become cell repellent, and the size of the spheroid will be directly proportionate to the microwell size. The hanging drop relies on the surface tension properties of cell loaded droplets which hang from a hard surface, causing the cells to sediment and aggregate. More advanced version of this system uses perforated substrates to increase throughput and better tune the size and load of each droplets [70].

In cancer research especially it has been demonstrated that the progression of the tumour involves complex mechanisms, but the abnormal cellular proliferation rate results in an amorphous structure, which therefore can be reproduced in vitro without too much regards for the cellular organisation. Such artificial tumours are called "Multi-Cellular Tumour Spheroids" (MCTS). First established in the early 70s [71], MCTS have been widely employed as models to study a great variety of phenomena, such as cell growth and proliferation, and cell response or resistance to drugs [72-74]. Compared to traditional 2D models, these MCTS can include multiple cell types, incorporate high cell-cell interactions, and possess a hypoxic/necrotic region depending on their size [75-77]. In this thesis, we use an ultra-low attachment microwell assay developed in our research group [78], as detailed in chapter 5, to generate MCTS.

\subsection{MICROFLUIDICS, A MINIATURIZED LABORATORY WITH LARGE POTENTIAL}

Microfluidics is the science of manipulating fluids in the range of the nanolitre $\left(10^{-9} \mathrm{~L}\right)$ down to the picolitre $\left(10^{-12} \mathrm{~L}\right)$ [79]. At those scales viscous forces are no longer negligible compared to inertial forces, and fluid behaviour changes, switching from a "chaotic" behaviour or "turbulent flow", towards an organized behaviour or "laminar flow" (Figure 1-5). In this laminar regime, the motion of particles in solution becomes predictable, allowing for the fine 
control of liquid manipulation, as well as particle sorting [80]. Additionally, at this scale, capillary forces become no longer negligible compared to gravity forces, which brings new opportunities for passive fluid control.

Because of the unique properties of flow at that scale, microfluidics can reproduce or even improve standard laboratory procedures (e.g., sorting, measuring), while using smaller volumes of samples, and providing faster readouts. To achieve this, fluids are transported in channels of a few hundreds of micrometres down to a few hundreds of nanometres, which are also known as micro- and nanofluidic channels. These channels can be fabricated in different materials (e.g., glass, plastic, silicon) [81, 82], which are often transparent for in situ optical readout. Additionally, these channels can comprise actuators (e.g., electro/magnetic field or piezoelectric generators) [83, 84] or sensors (e.g., oxygen, $\mathrm{pH}$, temperature) [85], and they can be connected to external systems for flow control (e.g., pressure pump, or syringe pump) and measurements (e.g., microscope, laser) [86].
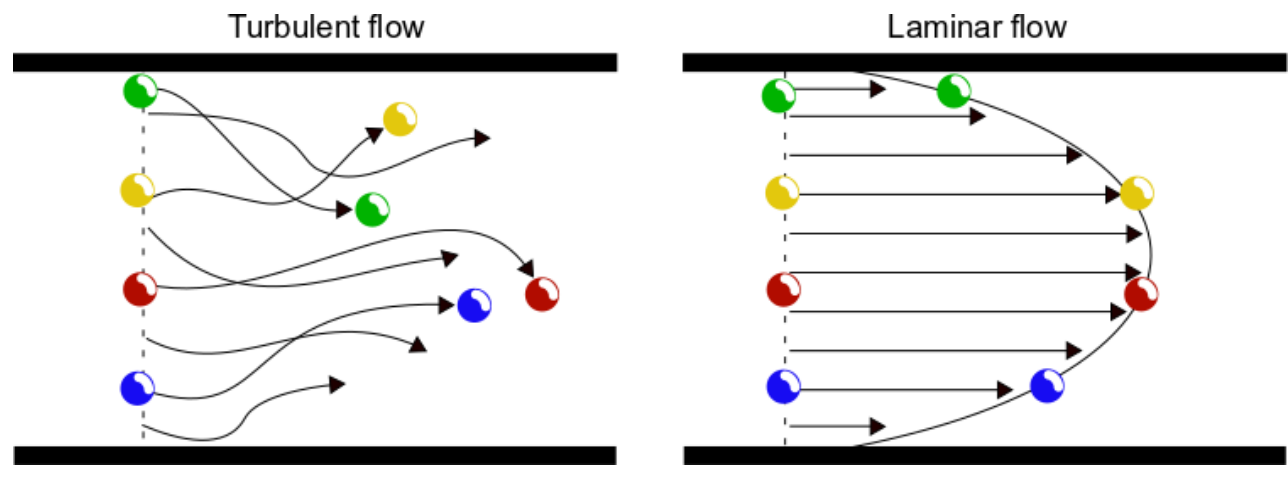

Figure 1-5 Particle motion in a turbulent flow and a laminar flow

Since microfluidics only became popular in the last thirty years in the scientific community, only few applications of microfluidics have successfully reached the market. The first commercialized applications of microfluidics are inkjet printers, developed in the 80s, allowing for high precision printing by generating highly tuned ink droplets $\left(\sim 10^{5}\right.$ droplets of 1-10 $\mathrm{pl}$ in size per second), a method still in use today [87]. A more recent achievement is the development of innovative diagnostic systems integrating microfluidic technology, and in which multiple analyses can be performed using very small amounts of bodily samples (e.g., urine, blood). As such, microfluidics has revolutionized the development of "point-of-care" (POC) devices, which allow doctors to run rapid diagnoses, without the need of expensive and often inaccessible equipment, and the necessary presence of a technical expert. It also allows patients to perform their own medical test, reducing the need to visit hospitals, and associated healthcare. Several companies (e.g., Philips, Abbott, Alere) have developed microfluidics- 
based POC devices, for whole blood analysis, or for certain diseases (e.g., diabetes, HIV/AIDS, Malaria) [88], performing analysis in record time. For instance, the Pima analyser by Abbott can, in only $20 \mathrm{~min}$, and using a single drop of blood $(\sim 5 \mu \mathrm{L})$, establish a baseline immunity of patient infected with HIV and determine the treatment required [89].

In academia, many different applications to microfluidics have been proposed, ranging from fundamental research (e.g., fluid behaviour/particle motion), to very applied studies. Microfluidics has especially become a powerful tool in molecular biology [90]. Among other applications, microfluidics has greatly improved the process of DNA replication, known as polymerase chain reaction (PCR), for the analysis of gene expression, and provided accurate and accelerated readouts on bacterial response to antibiotics [91, 92].

In cell biology, microfluidics is also used to generate in vitro models on chip. Unlike the standard 2D in vitro platforms, these monolayers on-chip are confined in a closed system, in which parameters like temperature or $\mathrm{O}_{2} / \mathrm{CO}_{2}$ levels, can be precisely tuned. Additionally, the confinement allows for a controlled continuous perfusion of media, limiting the need of daily refreshment [93]. Moreover the perfusion can be optimized to reproduce shear stress levels found in in vivo tissues (e.g., blood vessel shear stress) [94], on the cells present in the microfluidic device. Indeed, cells exposed to certain stresses have shown to change their morphology and behaviour [95, 96]. Finally, using parallelized systems (e.g., multiple chambers), microfluidics allows for high throughput drug screening, using the smallest amount of reagents (a few pl per chambers) [93, 97].

\subsection{ORGAN-ON-CHIP, MIMICKING IN VIVO ORGAN FUNCTIONS}

Another application of microfluidics in the field of biology, is the ability to reproduce biological functions found in vivo, by cultivating cells in a device able to mimic the architecture and/or function of targeted organs [98-100]. One of the very first advanced examples is the "lung-on-chip" platform, developed by the Wyss Institute [101]. This device comprised a stretchable porous membrane onto which epithelial cells were grown, mimicking the blood alveolar barrier. Breathing motion was implemented by periodically stretching the elastomeric membrane. These organ-model systems represent a potential alternative to the established Transwell as a platform for testing particle toxicity or medicines (Figure 1-6) [102]. 
These devices, called organ-on-chip systems, have now been developed for a wide range of organs, for various applications as reviewed by J. Sosa-Hernández et al. [100]. For instance, several blood brain barrier (BBB) on-chip models have been proposed, similarly to the lung on-chip, using a porous membrane and different cell types (i.e., endothelial cells, pericytes and astrocytes) [103-105]. In vivo, the blood vessels in the brain differ from the other blood vessels in the body, due to the combination of pericytes and astrocytes, which reinforce the endothelial barrier, and drastically limit the risk of infection but also the uptake of medicine [106]. These platforms could potentially support the development of drugs able to cross the $\mathrm{BBB}$, to treat some of the most challenging diseases of modern medicine (e.g., Glioblastoma, Alzheimer's, Parkinson's).

Several other organ functions have been reproduced on chip, following the lung-onchip model, such as a gut-on-chip containing villi structures, which increases the surface of absorption of the small intestine [107], a brain-on-chip recapitulating the neural network [108], or a heart-on-chip to study diseases like cardiac myopathy [109]. Additionally, work is being performed to bring together different organ-on-chip platforms together, to form complex biological functions (e.g., digestive system, circulatory system), and eventually a complex human-on-chip model. Such models could improve our knowledge on both pharmacokinetics and pharmacodynamics of medicines, and therefore help identify possible side-effects before even starting in vivo experiments.
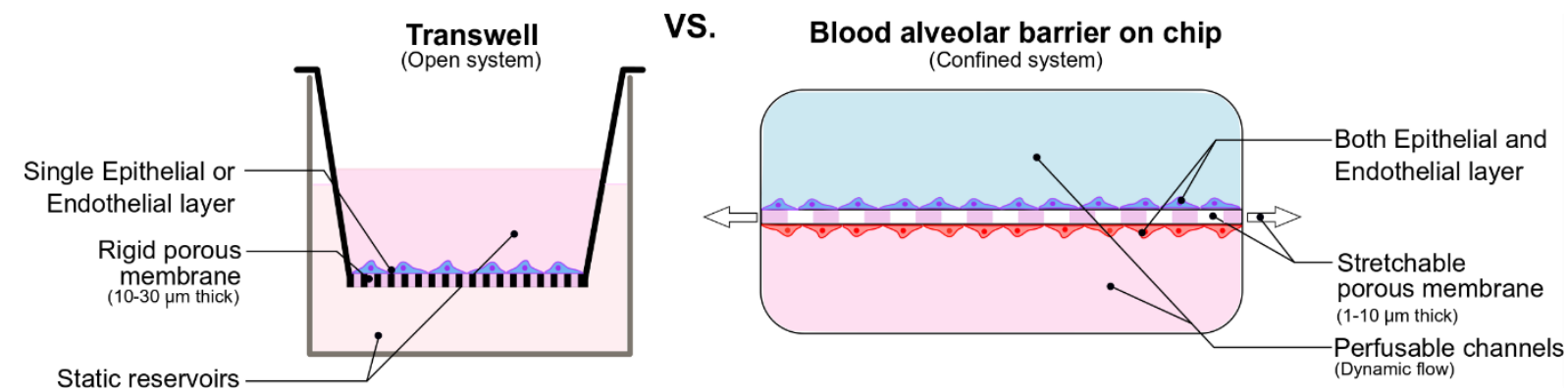

Figure 1-6 Comparison between the in vitro barrier systems Transwell and Organ-on-Chip. The Transwell system is a simple model easy-to-use, but lacks several elements present in the "On-Chip" barrier system: A perfusion system for the nutrient supply and generating shear stress on the cells, a stretchable membrane mimicking the breathing motion or blood vessel dilation, and more importantly the combination of an endothelial and epithelial layers, better approaching the in vivo tissue interface. 


\subsection{THE TUMOUR-ON-CHIP}

Microfluidics and organ-on chip technology have also been used to investigate tumour proliferation and response to medicines. The first and main method used to engineer this socalled "Tumour-on-Chip" model consisted in preparing or introducing tumour spheroids inside a microfluidic device with a structure adapted to ensure their retention inside the device [110113]. Such systems are on average easy to implement, high-throughput, and highly parallelized. For instance, Patra et al. developed a microfluidic device containing a straight channel containing a well array in which the cells could aggregate into harvestable spheroids [114]. Thanks to the confinement they offer, these microfluidic devices can be used to perform drug or toxicity assays in situ, while minimizing the amount of reagent necessary and maximizing interactions with the target tissue. Y. Chen et al. developed a tumour-on-chip platform in which spheroids were prepared (Figure 1-7.a), and on which photodynamic therapy (non-toxic reactive agents activated by light) was applied [115], revealing a better response to the treatment compared to traditional chemotherapy. Although simplified, these tumour-on-chip platforms have proven to be promising to recapitulate avascular cancerous (malignant) tumours, and as high throughput drug screening systems $[68,116,117]$.

a)

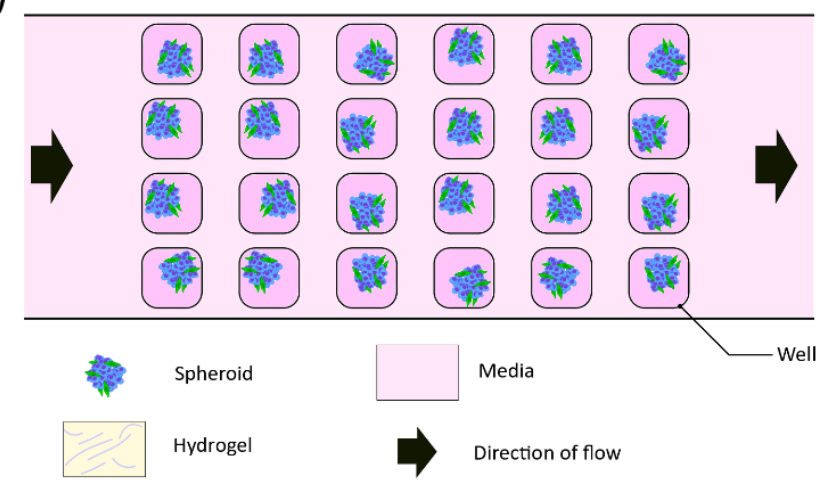

b)

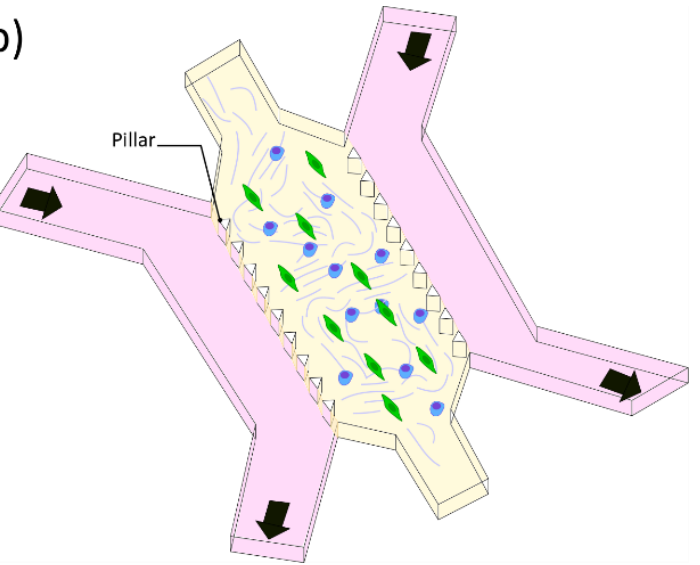

Figure 1-7 Examples of tumour-on-chip platforms. a) Schematic of a tumour-on-chip platform to generate and analyse tumour spheroid, as described by Chen et al. [112]. b) Example of tumour-onchip platform containing a cell-laden hydrogel separated from two perfusion channels by arrays of pillars [114].

Alternatively to the tumour spheroid array approach, microfluidics has been used to produce a more complex tumour microenvironment, by placing different cell types in a hydrogel material. The most established model is presented as a three channels system, separated by pillar arrays, in which cells in hydrogel are placed in the central channel, and 
nutrients and reagents are perfused in the lateral channels (Figure 1-7.b) [118]. Not unlike the tumour spheroid, this system presents some of the aforementioned key-features of a relevant TME model: it contains ECM, multiple cell types, and can emulate the gradients of oxygen and nutrients found in a tumour tissue in vivo. As such, it has been implemented in many different cancer studies $[108,119]$.

Finally, tumour-on-chip systems have been developed to integrate ex vivo tissues [114, 120]. Unlike in vitro 3D models like the tumour spheroid, these tissues contain an organized microarchitecture, which can play an important role on their response to exposure to drugs or infections.

\subsection{THE NEXT STEP OF THE ORGAN-ON-CHIP MODEL}

The tumour-on-chip models described above have already provided important information on cancer research, and can serve as powerful alternatives to the traditional 2D models. However, to this day, there has not been any standardization on the development of these models and most of them use materials not meeting the industry standard (e.g., polydimethylsiloxane). Moreover, several key features mentioned previously are still missing, which could further improve the tumour microenvironment model: the vascular network and the immune system. If the latter has not been considered yet in microfluidics, the former is currently being investigated and early models of vascularized tumour-on-chip have been published in the last years $[121,122]$. By providing the system with an accurate administration route, and the endothelial wall known to be a barrier to the penetration of the drugs, these models represent the next generation of tumour in vitro models.

\section{GOALS OF THE THESIS}

This thesis reports a series of experiments designed to better understand the in vitro 3D multicellular tumour spheroid, and with the help of microfluidics, improve on the model in order to present a novel platform for drug assays. Chapter $\mathbf{2}$ starts with a comparison between the 2D monolayer and different sizes of 3D MCTS models of mouse breast cancer cells, by analysing their total proteomic contents, and characterizing the expression of their signalling 
pathways and cancer-associated proteins. Chapter 3, increases the complexity of the in vitro model by integrating fibroblasts in the MCTS, and changes the characterization approach by focusing on the spatial location of a few chosen proteins in the spheroid, through the use of high-resolution imaging and tissue clearing techniques. Chapter 4 improves on the method developed in Chapter 3, and presents as a proof of concept a characterization of the variation in oxygen concentration inside the MCTS, as well as the secretion of ECM. Chapter 5 investigates the impact of fibroblasts in the MCTS on the barrier to nanomedicine penetration, and presents a tumour-on-chip platform designed to trap multiple MCTS and investigate the penetration of nanoparticles of different sizes and compositions. Finally, Chapter 6 aims to further improve the in vitro model, by translating to human cells and integrating a vascular system to the previously established MCTS, better recapitulating the in vivo environment. Chapter 7 presents a summary of the results obtained, as well as recommendations for future investigators.

\section{REFERENCES}

1. Bray, F., et al., Global cancer statistics 2018: GLOBOCAN estimates of incidence and mortality worldwide for 36 cancers in 185 countries. CA Cancer J Clin, 2018. 68(6): p. 394-424.

2. Matthews, H., J. Hanison, and N. Nirmalan, "Omics"-Informed Drug and Biomarker Discovery: Opportunities, Challenges and Future Perspectives. Proteomes, 2016. 4(3).

3. Cristofanilli, M., et al., Circulating tumor cells, disease progression, and survival in metastatic breast cancer. N Engl J Med, 2004. 351(8): p. 781-91.

4. $\quad$ Croce, C.M., Oncogenes and cancer. N Engl J Med, 2008. 358(5): p. 502-11.

5. Hodgson, S., Mechanisms of inherited cancer susceptibility. J Zhejiang Univ Sci B, 2008. 9(1): p. 1-4.

6. Sun, Y.S., et al., Risk Factors and Preventions of Breast Cancer. Int J Biol Sci, 2017. 13(11): p. 1387-1397.

7. Parsa, N., Environmental factors inducing human cancers. Iran J Public Health, 2012. 41(11): p. 1-9.

8. Kourou, K., et al., Machine learning applications in cancer prognosis and prediction. Comput Struct Biotechnol J, 2015. 13: p. 8-17.

9. Hanahan, D. and R.A. Weinberg, Hallmarks of cancer: the next generation. Cell, 2011. 144(5): p. 646-74.

10. Balkwill, F.R., M. Capasso, and T. Hagemann, The tumor microenvironment at a glance. Journal of Cell Science, 2012. 125(23): p. 5591-5596.

11. Kalluri, R. and M. Zeisberg, Fibroblasts in cancer. Nat Rev Cancer, 2006. 6(5): p. 392401. 
12. Kalluri, R., The biology and function of fibroblasts in cancer. Nat Rev Cancer, 2016. 16(9): p. 582-98.

13. Shimoda, M., K.T. Mellody, and A. Orimo, Carcinoma-associated fibroblasts are a ratelimiting determinant for tumour progression. Semin Cell Dev Biol, 2010. 21(1): p. 1925.

14. Alkasalias, T., et al., Fibroblasts in the Tumor Microenvironment: Shield or Spear? Int J Mol Sci, 2018. 19(5).

15. Maiullari, F., et al., A multi-cellular 3D bioprinting approach for vascularized heart tissue engineering based on HUVECs and iPSC-derived cardiomyocytes. Sci Rep, 2018. 8(1): p. 13532.

16. Orimo, A., et al., Stromal fibroblasts present in invasive human breast carcinomas promote tumor growth and angiogenesis through elevated SDF-1/CXCL12 secretion. Cell, 2005. 121(3): p. 335-348.

17. Hasebe, T., et al., Atypical tumor-stromal fibroblasts in invasive ductal carcinoma of the breast. Am J Surg Pathol, 2011. 35(3): p. 325-36.

18. Lee, J.H., H.M. Kim, and J.S. Koo, Differential Expression of Cancer-Associated Fibroblast-Related Proteins in Ductal Carcinoma in situ According to Molecular Subtype and Stromal Histology. Pathobiology, 2018. 85(5-6): p. 311-321.

19. Aboussekhra, A., Role of cancer-associated fibroblasts in breast cancer development and prognosis. Int J Dev Biol, 2011. 55(7-9): p. 841-9.

20. Eales, K.L., K.E. Hollinshead, and D.A. Tennant, Hypoxia and metabolic adaptation of cancer cells. Oncogenesis, 2016. 5: p. e190.

21. Al Tameemi, W., et al., Hypoxia-Modified Cancer Cell Metabolism. Front Cell Dev Biol, 2019. 7: p. 4.

22. Hicklin, D.J. and L.M. Ellis, Role of the vascular endothelial growth factor pathway in tumor growth and angiogenesis. J Clin Oncol, 2005. 23(5): p. 1011-27.

23. Carmeliet, P. and R.K. Jain, Angiogenesis in cancer and other diseases. Nature, 2000. 407(6801): p. 249-57.

24. Brzozowa, M., et al., The role of tumour microenvironment in gastric cancer angiogenesis. Prz Gastroenterol, 2014. 9(6): p. 325-8.

25. Hillen, F. and A.W. Griffioen, Tumour vascularization: sprouting angiogenesis and beyond. Cancer Metastasis Rev, 2007. 26(3-4): p. 489-502.

26. Nagy, J.A. and H.F. Dvorak, Heterogeneity of the tumor vasculature: the need for new tumor blood vessel type-specific targets. Clinical \& Experimental Metastasis, 2012. 29(7): p. 657-662.

27. Nagy, J.A., et al., Why are tumour blood vessels abnormal and why is it important to know? British Journal of Cancer, 2009. 100(6): p. 865-869.

28. Ha, T.Y., The role of regulatory T cells in cancer. Immune Netw, 2009. 9(6): p. 209-35.

29. Ruffell, B., et al., Lymphocytes in cancer development: polarization towards pro-tumor immunity. Cytokine Growth Factor Rev, 2010. 21(1): p. 3-10.

30. Obeid, E., et al., The role of tumor-associated macrophages in breast cancer progression. International Journal of Oncology, 2013. 43(1): p. 5-12.

31. Siveen, K.S. and G. Kuttan, Role of macrophages in tumour progression. Immunology Letters, 2009. 123(2): p. 97-102.

32. Dittmer, A., et al., Mesenchymal stem cells and carcinoma-associated fibroblasts sensitize breast cancer cells in 3D cultures to kinase inhibitors. International Journal of Oncology, 2011. 39(3): p. 689-696. 
33. Spaeth, E.L., et al., Mesenchymal Stem Cell Transition to Tumor-Associated Fibroblasts Contributes to Fibrovascular Network Expansion and Tumor Progression. Plos One, 2009. 4(4).

34. Harrison, L.B., et al., Impact of tumor hypoxia and anemia on radiation therapy outcomes. Oncologist, 2002. 7(6): p. 492-508.

35. Radiation Therapy: Effective for Prostate Cancer. 2015; Available from: https://www.targetingcancer.com.au/2015/02/radiation-therapy-95-effectiveprostate-cancer/.

36. Horsman, M.R. and J. Overgaard, The impact of hypoxia and its modification of the outcome of radiotherapy. J Radiat Res, 2016. 57 Suppl 1: p. i90-i98.

37. Sheehan, J.P., et al., Improving the radiosensitivity of radioresistant and hypoxic glioblastoma. Future Oncol, 2010. 6(10): p. 1591-601.

38. Scott, R.B., Cancer chemotherapy--the first twenty-five years. Br Med J, 1970. 4(5730): p. 259-65.

39. Saffhill, R., G.P. Margison, and P.J. Oconnor, Mechanisms of Carcinogenesis Induced by Alkylating-Agents. Biochimica Et Biophysica Acta, 1985. 823(2): p. 111-145.

40. Hickman, J.A., Apoptosis Induced by Anticancer Drugs. Cancer and Metastasis Reviews, 1992. 11(2): p. 121-139.

41. Vitug, A.F. and L.A. Newman, Complications in breast surgery. Surgical Clinics of North America, 2007. 87(2): p. 431-+.

42. Chatterjee, A., et al., Early postoperative outcomes in lumpectomy versus simple mastectomy. Journal of Surgical Research, 2015. 198(1): p. 143-148.

43. Quirke, V.M., Tamoxifen from Failed Contraceptive Pill to Best-Selling Breast Cancer Medicine: A Case-Study in Pharmaceutical Innovation. Frontiers in Pharmacology, 2017. 8.

44. Wu, L.M. and A. Amidi, Cognitive impairment following hormone therapy: current opinion of research in breast and prostate cancer patients. Curr Opin Support Palliat Care, 2017. 11(1): p. 38-45.

45. Wicki, A., et al., Nanomedicine in cancer therapy: challenges, opportunities, and clinical applications. J Control Release, 2015. 200: p. 138-57.

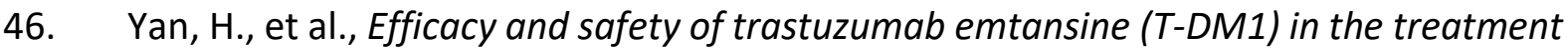
of HER2-positive metastatic breast cancer (MBC): a meta-analysis of randomized controlled trial. Oncotarget, 2017. 8(60): p. 102458-102467.

47. Kramer-Marek, G. and J. Capala, The role of nuclear medicine in modern therapy of cancer. Tumour Biol, 2012. 33(3): p. 629-40.

48. Biersack, H.J., et al., The role of nuclear medicine in oncology. Ann Nucl Med, 1992. 6(3): p. 131-6.

49. Calderon, M., et al., Functional dendritic polymer architectures as stimuli-responsive nanocarriers. Biochimie, 2010. 92(9): p. 1242-1251.

50. Sanna, V., N. Pala, and M. Sechi, Targeted therapy using nanotechnology: focus on cancer. International Journal of Nanomedicine, 2014. 9: p. 467-483.

51. Sekhon, B.S. and S.R. Kamboj, Inorganic nanomedicine-Part 1. NanomedicineNanotechnology Biology and Medicine, 2010. 6(4): p. 516-522.

52. Libutti, S.K., et al., Phase I and pharmacokinetic studies of CYT-6091, a novel PEGylated colloidal gold-rhTNF nanomedicine. Clin Cancer Res, 2010. 16(24): p. 6139-49.

53. Parveen, S. and S.K. Sahoo, Nanomedicine: clinical applications of polyethylene glycol conjugated proteins and drugs. Clin Pharmacokinet, 2006. 45(10): p. 965-88. 
54. Tang, H., et al., Recent Development of pH-Responsive Polymers for Cancer Nanomedicine. Molecules, 2018. 24(1).

55. Zhang, Y., et al., Temperature-dependent cell death patterns induced by functionalized gold nanoparticle photothermal therapy in melanoma cells. Sci Rep, 2018. 8(1): p. 8720.

56. Maeda, H., The enhanced permeability and retention (EPR) effect in tumor vasculature: the key role of tumor-selective macromolecular drug targeting. Adv Enzyme Regul, 2001. 41: p. 189-207.

57. Jain, R.K. and T. Stylianopoulos, Delivering nanomedicine to solid tumors. Nature Reviews Clinical Oncology, 2010. 7: p. 653.

58. Fang, J., H. Nakamura, and H. Maeda, The EPR effect: Unique features of tumor blood vessels for drug delivery, factors involved, and limitations and augmentation of the effect. Adv Drug Deliv Rev, 2011. 63(3): p. 136-51.

59. Mohs, R.C. and N.H. Greig, Drug discovery and development: Role of basic biological research. Alzheimers Dement (N Y), 2017. 3(4): p. 651-657.

60. Fogel, D.B., Factors associated with clinical trials that fail and opportunities for improving the likelihood of success: A review. Contemporary Clinical Trials Communications, 2018. 11: p. 156-164.

61. Duval, K., et al., Modeling Physiological Events in 2D vs. 3D Cell Culture. Physiology (Bethesda), 2017. 32(4): p. 266-277.

62. Mather, J.P., In vitro models. Stem Cells, 2012. 30(2): p. 95-9.

63. Katt, M.E., et al., In Vitro Tumor Models: Advantages, Disadvantages, Variables, and Selecting the Right Platform. Front Bioeng Biotechnol, 2016. 4: p. 12.

64. Srikanth, L., et al., Gel based in vitro 3D model exploring the osteocytic potentiality of human CD34(+) stem cells. Molecular Biology Reports, 2016. 43(11): p. 1233-1242.

65. Langer, K. and H.N. Joensson, Rapid Production and Recovery of Cell Spheroids by Automated Droplet Microfluidics. SLAS Technol, 2019: p. 2472630319877376.

66. Del Duca, D., T. Werbowetski, and R.F. Del Maestro, Spheroid preparation from hanging drops: characterization of a model of brain tumor invasion. J Neurooncol, 2004. 67(3): p. 295-303.

67. Vinci, M., et al., Advances in establishment and analysis of three-dimensional tumor spheroid-based functional assays for target validation and drug evaluation. BMC Biol, 2012. 10: p. 29.

68. Sant, S. and P.A. Johnston, The production of 3D tumor spheroids for cancer drug discovery. Drug Discov Today Technol, 2017. 23: p. 27-36.

69. Castaneda, F. and R.K. Kinne, Short exposure to millimolar concentrations of ethanol induces apoptotic cell death in multicellular HepG2 spheroids. J Cancer Res Clin Oncol, 2000. 126(6): p. 305-10.

70. Tung, Y.C., et al., High-throughput 3D spheroid culture and drug testing using a 384 hanging drop array. Analyst, 2011. 136(3): p. 473-8.

71. Sutherland, R.M., Cell and Environment Interactions in Tumor Microregions - the Multicell Spheroid Model. Science, 1988. 240(4849): p. 177-184.

72. Costa, E.C., et al., 3D tumor spheroids: an overview on the tools and techniques used for their analysis. Biotechnology Advances, 2016. 34(8): p. 1427-1441.

73. Inch, W.R., J.A. McCredie, and R.M. Sutherland, Growth of nodular carcinomas in rodents compared with multi-cell spheroids in tissue culture. Growth, 1970. 34(3): p. 271-82. 
74. Weiswald, L.B., D. Bellet, and V. Dangles-Marie, Spherical cancer models in tumor biology. Neoplasia, 2015. 17(1): p. 1-15.

75. Ishiguro, T., et al., Tumor-derived spheroids: Relevance to cancer stem cells and clinical applications. Cancer Sci, 2017. 108(3): p. 283-289.

76. Tsai, H.F., et al., Tumour-on-a-chip: microfluidic models of tumour morphology, growth and microenvironment. J R Soc Interface, 2017. 14(131).

77. Zanoni, M., et al., 3D tumor spheroid models for in vitro therapeutic screening: a systematic approach to enhance the biological relevance of data obtained. Scientific Reports, 2016. 6.

78. Sridhar, A., et al., Microstamped Petri dishes for scanning electrochemical microscopy analysis of arrays of microtissues. PLoS One, 2014. 9(4): p. e93618.

79. Kirby, B.J., Micro- and Nanoscale Fluid Mechanics: Transport in Microfluidic Devices. Cambridge University Press, 2010.

80. Guha, A., Transport and deposition of particles in turbulent and laminar flow. Annual Review of Fluid Mechanics, 2008. 40: p. 311-341.

81. Tsao, C.W. and D.L. DeVoe, Bonding of thermoplastic polymer microfluidics. Microfluidics and Nanofluidics, 2009. 6(1): p. 1-16.

82. McDonald, J.C., et al., Fabrication of microfluidic systems in poly(dimethylsiloxane). Electrophoresis, 2000. 21(1): p. 27-40.

83. Yeo, L.Y. and J.R. Friend, Surface Acoustic Wave Microfluidics. Annual Review of Fluid Mechanics, Vol 46, 2014. 46: p. 379-406.

84. Wen, C.Y., et al., Rapid magnetic microfluidic mixer utilizing AC electromagnetic field. Electrophoresis, 2009. 30(24): p. 4179-4186.

85. Gruber, P., et al., Integration and application of optical chemical sensors in microbioreactors. Lab Chip, 2017. 17(16): p. 2693-2712.

86. Whitesides, G.M., The origins and the future of microfluidics. Nature, 2006. 442(7101): p. 368-73.

87. Singh, M., et al., Inkjet Printing-Process and Its Applications. Advanced Materials, 2010. 22(6): p. 673-685.

88. Chin, C.D., V. Linder, and S.K. Sia, Commercialization of microfluidic point-of-care diagnostic devices. Lab Chip, 2012. 12(12): p. 2118-34.

89. St John, A. and C.P. Price, Existing and Emerging Technologies for Point-of-Care Testing. Clin Biochem Rev, 2014. 35(3): p. 155-67.

90. Velve-Casquillas, G., et al., Microfluidic tools for cell biological research. Nano Today, 2010. 5(1): p. 28-47.

91. Ahrberg, C.D., A. Manz, and B.G. Chung, Polymerase chain reaction in microfluidic devices. Lab Chip, 2016. 16(20): p. 3866-3884.

92. Dai, J., M. Hamon, and S. Jambovane, Microfluidics for Antibiotic Susceptibility and Toxicity Testing. Bioengineering (Basel), 2016. 3(4).

93. Beebe, D.J., G.A. Mensing, and G.M. Walker, Physics and applications of microfluidics in biology. Annu Rev Biomed Eng, 2002. 4: p. 261-86.

94. Egginton, S., In vivo shear stress response. Biochem Soc Trans, 2011. 39(6): p. 1633-8.

95. Armistead, F.J., et al., Cells Under Stress: An Inertial-Shear Microfluidic Determination of Cell Behavior. Biophysical Journal, 2019. 116(6): p. 1127-1135.

96. Potter, C.M.F., et al., Role of Shear Stress in Endothelial Cell Morphology and Expression of Cyclooxygenase Isoforms. Arteriosclerosis Thrombosis and Vascular Biology, 2011. 31(2): p. 384-U314. 
97. Regnault, C., D.S. Dheeman, and A. Hochstetter, Microfluidic Devices for Drug Assays. High Throughput, 2018. 7(2).

98. Ronaldson-Bouchard, K. and G. Vunjak-Novakovic, Organs-on-a-Chip: A Fast Track for Engineered Human Tissues in Drug Development. Cell Stem Cell, 2018. 22(3): p. 310324.

99. Esch, E.W., A. Bahinski, and D. Huh, Organs-on-chips at the frontiers of drug discovery. Nature Reviews Drug Discovery, 2015. 14(4): p. 248-260.

100. Sosa-Hernandez, J.E., et al., Organs-on-a-Chip Module: A Review from the Development and Applications Perspective. Micromachines, 2018. 9(10).

101. Huh, D., et al., Reconstituting Organ-Level Lung Functions on a Chip. Science, 2010. 328(5986): p. 1662-1668.

102. Mondrinos, M.J., et al., Smoking Lung-on-a-chip: A Tissue-engineered Microphysiological Model of Cigarette Smoke-induced Airway Disease. Tissue Engineering Part A, 2015. 21: p. S40-S40.

103. Prabhakarpandian, B., et al., SyM-BBB: a microfluidic blood brain barrier model. Lab on a Chip, 2013. 13(6): p. 1093-1101.

104. Wevers, N.R., et al., A perfused human blood-brain barrier on-a-chip for highthroughput assessment of barrier function and antibody transport. Fluids and Barriers of the Cns, 2018. 15.

105. Griep, L.M., et al., BBB ON CHIP: microfluidic platform to mechanically and biochemically modulate blood-brain barrier function. Biomedical Microdevices, 2013. 15(1): p. 145-150.

106. Daneman, R. and A. Prat, The Blood-Brain Barrier. Cold Spring Harbor Perspectives in Biology, 2015. 7(1).

107. Shim, K.Y., et al., Microfluidic gut-on-a-chip with three-dimensional villi structure. Biomedical Microdevices, 2017. 19(2).

108. Osaki, T., V. Sivathanu, and R.D. Kamm, Engineered 3D vascular and neuronal networks in a microfluidic platform. Scientific Reports, 2018. 8.

109. Kitsara, M., et al., Heart on a chip: Micro-nanofabrication and microfluidics steering the future of cardiac tissue engineering. Microelectronic Engineering, 2019. 203: p. 4462.

110. Sun, D.P., et al., A novel three-dimensional microfluidic platform for on chip multicellular tumor spheroid formation and culture. Microfluidics and Nanofluidics, 2014. 17(5): p. 831-842.

111. Kim, T., I. Doh, and Y.H. Cho, On-chip three-dimensional tumor spheroid formation and pump-less perfusion culture using gravity-driven cell aggregation and balanced droplet dispensing. Biomicrofluidics, 2012.6(3).

112. Ziolkowska, K., et al., Long-term three-dimensional cell culture and anticancer drug activity evaluation in a microfluidic chip. Biosens Bioelectron, 2013. 40(1): p. 68-74.

113. Kwapiszewska, K., et al., A microfluidic-based platform for tumour spheroid culture, monitoring and drug screening. Lab Chip, 2014. 14(12): p. 2096-104.

114. Xiao, Y., et al., Ex vivo Dynamics of Human Glioblastoma Cells in a Microvasculatureon-a-Chip System Correlates with Tumor Heterogeneity and Subtypes. Adv Sci (Weinh), 2019. 6(8): p. 1801531.

115. Chen, Y.C., et al., High-Throughput Cancer Cell Sphere Formation for Characterizing the Efficacy of Photo Dynamic Therapy in 3D Cell Cultures. Sci Rep, 2015. 5: p. 12175. 
116. Wlodkowic, D. and J.M. Cooper, Tumors on chips: oncology meets microfluidics. Current Opinion in Chemical Biology, 2010. 14(5): p. 556-567.

117. Dhiman, N., et al., On-chip anticancer drug screening - Recent progress in microfluidic platforms to address challenges in chemotherapy. Biosens Bioelectron, 2019. 137: p. 236-254.

118. Ayuso, J.M., et al., Development and characterization of a microfluidic model of the tumour microenvironment. Sci Rep, 2016. 6: p. 36086.

119. Sontheimer-Phelps, A., B.A. Hassell, and D.E. Ingber, Modelling cancer in microfluidic human organs-on-chips. Nat Rev Cancer, 2019. 19(2): p. 65-81.

120. Nguyen, M., et al., Dissecting Effects of Anti-cancer Drugs and Cancer-Associated Fibroblasts by On-Chip Reconstitution of Immunocompetent Tumor Microenvironments. Cell Rep, 2018. 25(13): p. 3884-3893 e3.

121. Wang, X., Q. Sun, and J. Pei, Microfluidic-Based 3D Engineered Microvascular Networks and Their Applications in Vascularized Microtumor Models. Micromachines (Basel), 2018. 9(10).

122. Oh, S., et al., "Open-top" microfluidic device for in vitro three-dimensional capillary beds. Lab Chip, 2017. 17(20): p. 3405-3414. 


\section{Chapter 2 - Multicellular tumour spheroid proteomic analysis: how size influences protein expression on breast cancer cells}

\section{Abstract}

In this chapter we investigated how size influences the physiology of monoculture (4T1) multicellular tumour spheroids (MCTS) models. This analysis was performed by comparing their protein expression profiles, and against the established 2D monolayer model, using the nanoLC-MS/MS label-free quantification approach to identify relative significant variations. When comparing the 2D monolayers and 3D MCTS, results indicated a significant increase in protein expression linked to signaling pathways found activated in tumours in vivo (glycolysis, metabolic pathways, biosynthesis of amino acids), but also in pathways involved in the cytoskeleton reorganization (Tes, Tmsb4x, Crip1). When focusing on the most up-regulated proteins in the MCTS, several have been previously identified as promotors of the tumour progression in mouse or human breast cancer, and as potential targets for therapeutic treatments (Acs14, Tmsb4x, Spp1, Casp3, Tgm2, Hmga2, Cstb, Crip1). We also studied whether the size of the tumour spheroids would affect this protein expression profile. When increasing the spheroid size from $\mathrm{ca}$. 200 to $350 \mu \mathrm{m}$ in diameter, an activation of hypoxic pathways and a decrease in cell motility pathways were observed. Certain proteins involved in cancer progression (Timm44, Aldh18a1, Aldh3a1, Tpp2) were overexpressed but Epithelial cell adhesion molecule (EpCAM), a key biomarker identified as overexpressed in breast cancer metastasis, became down regulated. These results not only confirmed the relevance of the 3D in vitro model (e.g., for drug assays), but also underlined how small variations in size of the MCTS can affect the activation of certain signaling pathways. Finally, while the MCTS represent an improvement to the $2 \mathrm{D}$ monolayer as a platform for biological assays, the disparities still observed with established breast cancer studies calls for refined 3D tumour models. 


\section{INTRODUCTION}

The still most commonly used in vitro model for drug or toxicity testing or to conduct basic research consists of a monolayer of cells grown in a plastic dishware [1]. This oversimplified cellular model is however not representative of the in vivo situation, in which cells exhibit very different shapes and structures compared to this $2 \mathrm{D}$ configuration and experience different cell-cell and cell-ECM (extracellular matrix) interactions. Furthermore, in a $3 \mathrm{D}$ configuration, there exist gradients of nutrients, oxygen and waste products, as a result of limitations in mass transport across the tissues, and these gradients impact the cell phenotype, behaviour and response to therapeutic treatment. Altogether, cell monolayers are no proper models for drug testing, and as a severe consequence for the pharmaceutical industry, drugs that show promising results when tested on cell monolayers fail at the pre-clinical and clinical phases when being evaluated on animal models and/or patients [2, 3]. Alternatively, different types of 3D cellular models have been proposed: either by growing cells in a 3D matrix (e.g., agarose, PEG (Polyethylene glycol), Matrigel ${ }^{\circ}$, collagen, etc.) [4], or by forcing cell aggregation into multicellular spheroids (MCTS) [5]. The latter spheroid models are particular attractive to represent avascular tumours that also present these essential gradients in nutrients and oxygen and, in turn, different cellular phenotypes, as found in tumour tissues in vivo [6].

Still, while it is now widely acknowledged that MCTS are more physiologically relevant in vitro models, one can wonder which characteristics a good 3D in vitro model should exhibit for drug testing on tumour tissues. Arguably, size matters: a size of several 100's of microns is required to emulate the different "layers" of proliferating, quiescent, and hypoxic/necrotic cells found in tumour tissues in vivo [7], and which all present very different responses to therapeutic treatment $[8,9]$. Notably, cells in a hypoxic environment are known to be resistant to chemotherapy [10] and radiotherapy [11], and, as such, they must be included to yield faithful 3D models. As a direct consequence, spheroids should present a narrow size distribution not to introduce any bias in the drug screening assay, a criterion that most of the traditional approaches forming spheroids (e.g., forced floating, spinning flask, rotating vessel) do not meet [12]. In contrast, microfabricated and microfluidic platforms allow controlling and tuning the spheroid size, and large-scale production [13]; these approaches including for instance microwell arrays [14, 15], hanging-drop culture [9], micropatterning [16], microchannels [17], droplet microfluidics [18, 19], anchored droplet arrays [20, 21], or, 
alternatively, the use of active means to promote cellular aggregation such as magnetic [22] or acoustic forces [23], or dielectrophoresis [24]. A second important parameter is the "age" of the spheroids as it also affects the cell phenotype: typically, cells require few days of culture to switch from the exponential growth behavior they exhibit in $2 \mathrm{D}$, to a more metabolic equilibrium phase, which characterizes a 3D culture [25-27]. Finally, for drug testing on tumour tissues, ideally different cell types must be incorporated to fully emulate the tumour microenvironment that also comprises immune and stromal cells (e.g., cancer-associated fibroblasts), next to the tumour cell [28]. Importantly, the presence of all these cell types not only influence the tumour response to the drug, but also the properties of the tumour tissue, and as such the penetration of the drugs and/or nanomedicines into the tissue [29, 30]. None of these essential parameters can be recapitulated in a $2 \mathrm{D}$ model, which further emphasizes the importance of 3D models for drug testing.

A variety of studies have characterized in vitro cellular models and examined differences, mostly between 2D and 3D models, at the molecular and phenotypic levels. Initially, mRNA gene expression profiles were analyzed [31,32], taking advantage of nucleic acid amplification techniques. However, mRNA expression levels do not fully mirror protein expression levels, the latter being the molecular key-players in a cell, tissue or organism. Furthermore, there exist additional levels of regulation at the protein level, such as the addition of PTMs (post-translational modifications), protein cleavage and/or activation, as well as the existence of different protein isoforms [33-35]. A handful of reports have focused on comparing protein expression between monolayers and spheroids [36-45]. Traditionally, comparative studies have been conducted using 2D SDS-PAGE (sodium dodecyl sulfatepolyacrylamide gel electrophoresis) followed by in-gel protein digestion and identification of one or few proteins of interest by MALDI-MS [36-38]. In a more refined version, DIGE has been employed for protein staining in the gel $[39,40]$. More recently, advances in the fields of proteomics and MS analysis have allowed more comprehensive studies using a bottom-up proteomic approach and differential labeling such as SILAC [41, 42], iTRAQ [43], isotopic or dimethyl labeling [44]. In a last very elegant approach, McMahon et al. characterized the structure between different layers of $850-\mu \mathrm{m}$ sized multicellular spheroids using progressive trypsin digestion and iTRAQ staining for differential analysis [45]. Because of the aforementioned potential of tumour spheroidd as novel in vitro models, several proteomics studies have also focused on answering biological questions previously unattainable with 2D models [46-48]. Feist et al. analysed using proteomics the decrease in abundance of H3K27me3 
enzyme, in colon cancer tumour spheroid exposed to FDA approved autophagy inducer UNC1999, and observed a correlation between the protein knockdown and a shrinkage of the spheroids, varying with the exposure conditions, in accordance with the in vivo situation [48]. However, so far, no study has systematically examined differences in protein expression profiles in spheroids of different sizes, since actually, in each aforementioned study, the size of the 3D cellular models was not accurately controlled.

Here, in an effort to further characterize the MCTS in vitro model as a platform for drug or toxicity assay, a study of the influence of the spheroid size was performed comparing of MCTS prepared from 4T1 mouse breast cancer cells, against the same cells cultured in 2D. First, homogeneously sized MCTS were prepared using an in-house developed microwell array and different microwell dimensions (width $\mathrm{x}$ height: $200 \mu \mathrm{m} \times 100 \mu \mathrm{m} ; 400 \mu \mathrm{m} \times 200 \mu \mathrm{m}$; and $800 \mu \mathrm{m} \times 500 \mu \mathrm{m})$, and characterized in terms of size and shape. Next, the protein content of MCTS of three different sizes (herein referred to as small, medium and large MCTS) and of conventional monolayer cultures were analyzed using nanoLC-MS/MS and a label-free quantification approach. Following this, the differences in protein expression profiles were studied using MaxQuant, Perseus and Morpheus software, on one hand, between 2D and 3D cultures and, on the other hand, between MCTS of different sizes. Changes in protein expression were quantified by identifying the different signaling pathways activated (gene ontology and KEGG), as well as by focusing on specific proteins whose expression levels have previously been established to be cancer-dependent. Characteristics of the different 2D and 3D biological conditions were compared based on the similarity of their protein expression profiles with previously reported profiles in in vitro and in vivo studies. 


\section{MATerials AND MethodS}

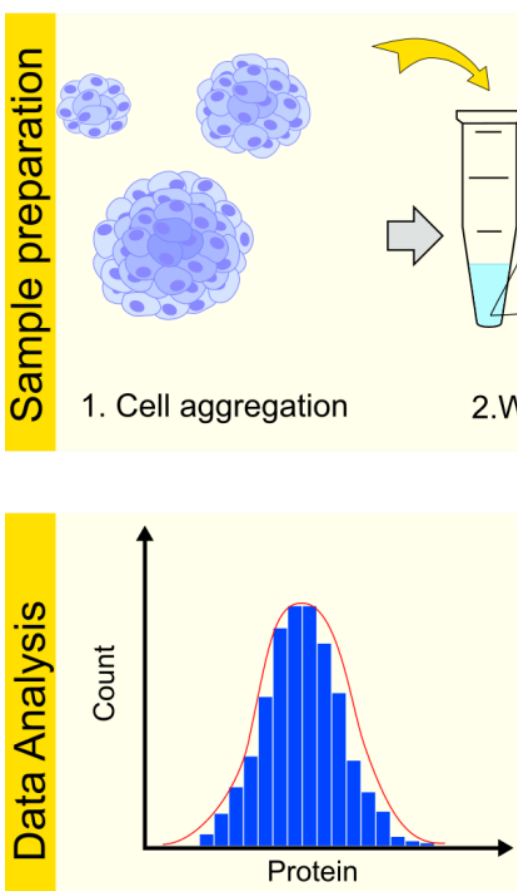

4. Label Free Quantification

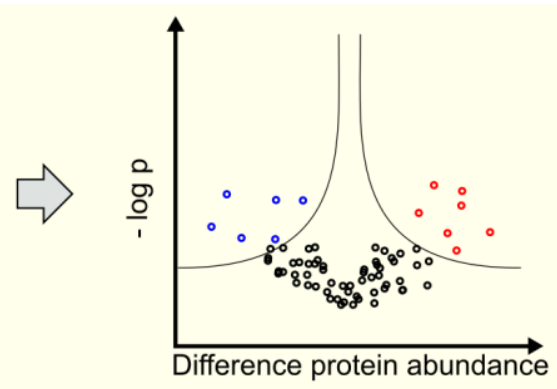

5. Stastical analysis

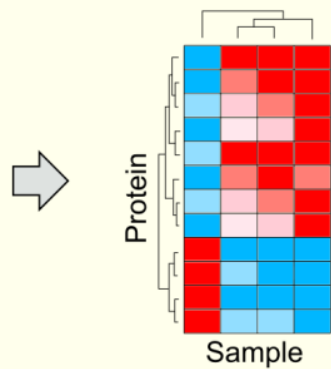

6. Biological analysis

Figure 2-8 Workflow for the analysis of MCTS protein expression profiles. (1) Cells are aggregated in microwell arrays, to yield three different sizes of MCTS. (2) MCTS and monolayer are digested to extract proteins (3) Proteins common to all samples are identified from the peptide sequences analyzed using LC MS/MS. (4) Label-free quantification identifies protein with significant variance (5). A threshold value focuses the analysis on the proteins and signaling pathways presenting the highest variations between conditions. (6) Comparison of the protein expression profiles is performed between $2 D$ and $3 D$ models, then between MCTS of three different sizes.

\subsection{Cell Culture}

4T1 mouse breast cancer cells were purchased from the America Type Culture Collection (ATCC, Rockville, MD, USA), and cultured in Roswell Park Memorial Institute (RPMI) 1640 medium, supplemented with $2 \mathrm{mM}$ L-glutamine, 10\% fetal bovine serum (FBS) and antibiotics (50 U.mL ${ }^{-1}$ penicillin and $50 \mathrm{ng} \cdot \mathrm{mL}^{-1}$ streptomycin). Cells were expanded in 75- $\mathrm{cm}^{2}$ culture flasks. (CELLSTAR® Filter Cap 75 $\mathrm{cm}^{2}$, GREINER BIO-ONE, Kremsmünster, Austria) in an incubator under standard humidified conditions $\left(37^{\circ} \mathrm{C}, 5 \% \mathrm{CO}_{2}\right)$. Medium was refreshed every 2 days. To retrieve the cells from the flask, medium was removed, and cells washed with sterile 1X Phosphate Buffer Saline (PBS) buffer (Lonza, Basel, Switzerland) to remove dead (floating) cells. $2 \mathrm{~mL}$ of trypsin-EDTA $(0.25 \%)$ (ThermoFisher Scientific, 
Waltham, MA, USA) was added for 5 min to detach the cells from the surface. The cells were next re-suspended in $8 \mathrm{~mL}$ of fresh DMEM medium to block the action of the trypsin, and collected in $10 \mathrm{~mL}$ tubes. After centrifugation at $300 \mathrm{~g}$ for $3 \mathrm{~min}$, medium was removed and 2 $\mathrm{mL}$ of fresh DMEM medium was added. Cells were counted, and ca. 2 million cells were seeded in a new culture flask. The remaining cells were kept for further experiments.

\subsection{Biological Sample Preparation}

\subsubsection{Monolayer}

Monolayer samples were prepared by seeding 2 million $4 \mathrm{~T} 1$ cells in $75-\mathrm{cm} 2$ culture flasks. After 5 days of culture or at $80 \%$ confluency, while refreshing medium every 2 days, cells were collected in a $10 \mathrm{~mL}$ tube, and centrifuged (300 g, 3min). The supernatant was discarded, and cells washed with $1 \mathrm{~mL}$ of $1 \mathrm{X}$ PBS to remove the excess of FBS and centrifuged again. Cells were pipetted in a $1.5 \mathrm{~mL}$ Eppendorf tube, centrifuged, and the supernatant was removed again. Cultures were frozen at $-80^{\circ} \mathrm{C}$ until sample preparation. Two biological replicates of the monolayer sample were prepared, containing each the cell content of one 75$\mathrm{cm}^{2}$ culture flask.

\subsubsection{Spheroid Preparation}

Multicellular tumour spheroids were prepared as previously reported by us, and briefly recalled hereafter [15]. Array of cylindrical microwells, with varying dimensions $(200 \mu \mathrm{m}$ diameter $\times 100 \mu \mathrm{m}$ depth, $400 \mu \mathrm{m}$ diameter $\times 200 \mu \mathrm{m}$ depth, and $800 \mu \mathrm{m}$ diameter $\times 500 \mu \mathrm{m}$ depth) were soft-embossed in $35 \mathrm{~mm}$ diameter Petri dishes (Greiner Bio-One, Kremsmünster, Austria), using a polydimethylsiloxane (PDMS) mold and a home-made set-up. The resulting Petri dishes were sterilized with a $70 \%$ ethanol aqueous solution and incubated overnight with a $1 \%$ w/w aqueous Pluronic F-127 solution (Sigma-Aldrich, Zwijndrecht, The Netherlands). Pluronic was replaced with $2 \mathrm{~mL}$ of fresh RPMI medium. About 2.5 million 4T1 cells were added in each Petri dish and forced into the wells by centrifugation (1800 g, $5 \mathrm{~min})$. The excess of cells was removed with a washing step with PBS, before $2 \mathrm{~mL}$ of fresh RPMI medium was added per dish. After $c a .24 \mathrm{~h}$, uniformly sized spheroids formed in every microwell (Figure 2-1.1). The spheroids were kept in culture in the devices for up to 5 days, while refreshing medium every 2 days. The resulting MCTS were washed twice with $1 \mathrm{X}$ PBS to remove the excess of FBS, since the latter would interfere with the protein analysis, and collected in a 1.5 $\mathrm{mL}$ Eppendorf microtubes (Eppendorf $\mathrm{GmbH}$, Hamburg, Germany). The spheroids were 
frozen at $-80^{\circ} \mathrm{C}$ until further sample preparation. For each spheroid size condition, two biological replicates were prepared.

\subsubsection{Size and shape characterization}

To characterize the size and shape of the MCTS, spheroids of each condition were first fixed by incubating them in a $4 \%$ formaldehyde solution for $1 \mathrm{~h}$. After which they were encased in cubes of agarose gel and imaged from different angles. To do so, a 3\% w/w agarose solution was prepared, by dissolving agarose powder (Ultra-Pure Agarose, Thermofisher Scientific) in distilled water at $70^{\circ} \mathrm{C}$. Fixed spheroids were mixed with the agarose before its gelation and poured in a 6-well plate (Corning). After complete agarose gelation $\left(30 \mathrm{~min}\right.$ at $20^{\circ} \mathrm{C}$ ), cubes of agarose containing a single spheroid each were cut. Images of the different faces of the cubes were taken (one in each dimension), using an EVOS-FL microscope (Thermofisher Scientific), and the diameter and shape of the spheroids in each dimension were measured using ImageJ software (NIH, Bethesda, MA, USA).

\subsection{Protein SAMPle PREPARATION}

\subsubsection{Extraction of proteins}

All solutions were prepared using ultrapure water purified with a MilliQ ${ }^{\mathrm{TM}}$ Academic system (Merck Millipore, Burlington, MA, USA). $300 \mu \mathrm{L}$ of cell lysis buffer (6 M urea, 50 mM Tris $\mathrm{HCl}, 150 \mathrm{mM} \mathrm{NaCl}, 1 \%$ IGEPAL CA-630 (octylphenoxypolyethoxyethanol), $1 \%$ sodium deoxycholate, $\mathrm{pH}$ 8.0) with complete protease inhibitor was added to the different cultures after thawing during $1 \mathrm{~h}$ at $4{ }^{\circ} \mathrm{C}$, and were subsequently frozen at $-80^{\circ} \mathrm{C}$ for $1 \mathrm{~h}$. The samples were next sonicated at $20 \mathrm{kHz}$ on ice with a Sonifier (BANDELIN SONOPULS HD2070, Berlin, Germany), four times for $30 \mathrm{~s}$ with a $10 \mathrm{~s}$ of break, until complete cell lysis was achieved (Figure 2-1.2). The lysates were incubated on ice for $30 \mathrm{~min}$, to ensure proper protein solubilization, and next centrifuged at $10,000 \mathrm{~g}$ for $30 \mathrm{~min}$ in a benchtop centrifuge (Beckman Coulter Allegra ${ }^{\mathrm{TM}}$ 64R Centrifuge, Brea, CA, USA) at $4^{\circ} \mathrm{C}$ to spheroid the cell debris. The supernatant was used for protein quantification. Protein quantification was performed according to the manufacturer's instructions for microplates with a BCAssay (ThermoFisher Scientific, Pierce ${ }^{\mathrm{TM}}$ BCA Protein Assay Kit). The absorbance values were read on an ELISA absorbance reader at $595 \mathrm{~nm}$ (Berthold APOLLO 11 LB 913, Calmbacher, Germany). $100 \mu \mathrm{L}$ of lysis buffer ( $8 \mathrm{M}$ urea, 4\% SDS (sodium dodecyl sulfate), $0.2 \%$ DCA (deoxycholic acid), $50 \mathrm{mM}$ DTT (dithiothreitol), and $100 \mathrm{mM}$ ammonium bicarbonate $\mathrm{pH} 8.8$ ) was added to a solution of $100 \mu \mathrm{g}$ of proteins, and the resulting mixture was incubated at $4^{\circ} \mathrm{C}$ overnight. 


\subsubsection{Digestion of samples}

Samples were next prepared using the eFASP (enhanced Filter Aided Sample Preparation) method [49]. Before their use, $0.5 \mathrm{~mL}$ Amicon ${ }^{\circledR}$ ultra centrifugal filters with a cutoff of $10 \mathrm{kDa}$ (EMD Millipore, Darmstadt, Germany) were incubated overnight with a passivation solution containing 5\% (v/v) Tween ${ }^{\circledR}-20$, and next rinsed with ultrapure water. 100 $\mu \mathrm{g}$ of cell lysate was transferred to an Amicon ${ }^{\circledR}$ filter, followed by $100 \mu \mathrm{L}$ of exchange buffer (8 $\mathrm{M}$ urea, $0.2 \%$ dichloroacetic acid (DCA), $100 \mathrm{mM}$ ammonium bicarbonate $\mathrm{pH} 8.8$ ). After a centrifugation step for $30 \mathrm{~min}$ at $10,000 \mathrm{~g}$, the filtrate was removed. $200 \mu \mathrm{L}$ of exchange buffer was next added to the Amicon ${ }^{\circledR}$ filter, which was again centrifuged. This operation was repeated twice in total. The proteins were alkylated for $1 \mathrm{~h}$ at room temperature in the dark using $100 \mu \mathrm{l}$ of alkylation buffer ( $8 \mathrm{M}$ urea, $50 \mathrm{mM}$ iodoacetamide, and $100 \mathrm{mM}$ ammonium bicarbonate, $\mathrm{pH} 8.8$ ). The Amicon ${ }^{\circledR}$ filter was centrifuged again for $30 \mathrm{~min}$ at $10,000 \mathrm{~g}$ and the filtrate discarded. After this alkylation step, $200 \mu \mathrm{L}$ of exchange buffer was added to the Amicon ${ }^{\circledR}$ filter, which was again centrifuged for $30 \mathrm{~min}$ at $10,000 \mathrm{~g}$, and the filtrate discarded. $200 \mu \mathrm{L}$ of digestion buffer ( $0.2 \%$ DCA, $50 \mathrm{mM}$ ammonium bicarbonate $\mathrm{pH} 8.8)$ was added to the Amicon ${ }^{\circledR}$ filter, before another centrifugation step (30 min at 10,000 g). This operation was repeated twice, the filtrate being removed and discarded. The Amicon ${ }^{\circledR}$ filter was transferred to a new $2 \mathrm{~mL}$ concentrator collection tube. $100 \mu \mathrm{L}$ of digestion buffer with $40 \mu \mathrm{L}$ of trypsin / LysC (Promega, Madison, WI, USA) was added and incubated in the Amicon ${ }^{\circledR}$ filter while shaking in a heating block tube (MHR23, Hettich, The Netherlands) overnight at $37^{\circ} \mathrm{C}$. Thereafter, the peptides present in the Amicon $^{\circledR}$ filter were recovered in the tube by centrifugation for $15 \mathrm{~min}$ at $10,000 \mathrm{~g}$. To maximize the peptide recovery, two washing steps were implemented with $50 \mu \mathrm{L}$ of ammonium bicarbonate solution $(50 \mathrm{mM} \mathrm{pH} 8.8)$. The filtrate containing all peptides was next transferred to a $1.5 \mathrm{~mL}$ Eppendorf ${ }^{\circledR}$ microtube (Eppendorf). $200 \mu \mathrm{L}$ of ethyl acetate with $2.5 \mu \mathrm{L}$ of trifluoroacetic acid were added, causing the peptide to precipitate (white color). At once, $800 \mu \mathrm{L}$ of ethyl acetate was added again, the resulting solution centrifuged for $10 \mathrm{~min}$ at $10,000 \mathrm{~g}$ and the organic phase eliminated. This operation was repeated twice. The Eppendorf ${ }^{\circledR}$ microtube was placed for $5 \mathrm{~min}$ at $60^{\circ} \mathrm{C}$ in a heating block (SBH130, Stuart, Staffordshire, UK) to let the remaining ethyl acetate evaporate. The samples were dried at room temperature in a SpeedVac ${ }^{\mathrm{TM}}$ Concentrator (Eppendorf ${ }^{\mathrm{TM}}$ Concentrator Plus, Eppendorf). Next, $100 \mu \mathrm{L}$ of a methanol / water (50/50) mixture was added to the resulting solid phase and let to evaporate. For MS analysis, the samples were dissolved in 10 $\mu \mathrm{L}$ of ultrapure water supplemented with $0.1 \%$ of formic acid. The sample concentration was 
estimated by measuring the OD (optical density) at $215 \mathrm{~nm}$ of $1 \mu \mathrm{L}$ of the solution using a droplet UV spectrometer (DS-11+, Denovix, Wilmington, DE, USA). Finally, the concentration of the sample was adjusted to $1 \mu \mathrm{g} / \mu \mathrm{L}$ by dilution with ultrapure water containing $0.1 \%$ formic acid (FA) before analysis.

\subsection{Protein Analysis}

\subsubsection{NanoLC-MS/MS analysis.}

NanoLC-MS/MS protein analysis was performed on an Orbitrap Q Exactive plus Mass Spectrometer hyphenated to a U3000 RSLC Microfluidic HPLC System (ThermoFisher Scientific) (Figure 2-1.3). $1 \mu \mathrm{L}$ of the peptide mixture at a concentration of $1 \mu \mathrm{g} . \mu \mathrm{L}^{-1}$ was injected with a solution A (5\% v/v acetonitrile and $0.1 \%$ formic acid) for $3 \mathrm{~min}$ at a flow rate of $10 \mu \mathrm{L} \cdot \mathrm{min}^{-1}$ on an Acclaim PepMap100 C18 pre-column $(5 \mu \mathrm{m}, 300 \mu \mathrm{m}$ i.d. $\times 5 \mathrm{~mm})$ (ThermoFisher Scientific). The peptides were next separated on a C18 Acclaim PepMap100 C18 reverse-phase column $(3 \mu \mathrm{m}$, dimensions $75 \mu \mathrm{m}$ i.d. $\times 500 \mathrm{~mm}$ long) (ThermoFisher Scientific), using a linear gradient (5-40\%) of solution B (75\% acetonitrile and $0.1 \%$ formic acid) using a flow-rate of $250 \mathrm{~nL}$.min-1 in 160 min followed by $100 \%$ solution B for 5 min. The column was regenerated by washing it for $5 \mathrm{~min}$ with solution $\mathrm{B}$ and then re-equilibrated with solution A. The column and the pre-column were placed in an oven at a temperature of $45^{\circ} \mathrm{C}$, reducing the eluent viscosity and improving the separation, for the total duration of the analysis (180 $\mathrm{min}$ ). The LC (liquid chromatography) runs were acquired in positive ion mode with MS scans from $\mathrm{m} / \mathrm{z} 350$ to 1,500 in the Orbitrap mass analyzer with a 70,000 resolution. The automatic gain control was set at $10^{6}$. MS/MS scans were sequentially acquired in the high-energy collision dissociation cell for the 15 most-intense ions detected in the full MS survey scan. Automatic gain control was set at $5 \times 10^{5}$, and the normalized collision energy was set to $28 \mathrm{eV}$. Dynamic exclusion was set at $90 \mathrm{~s}$ and ions with 1 and more than 8 charges were excluded. For each biological sample, three independent analytical run replicates were performed.

\subsubsection{Bioinformatics quantification}

Analysis of the raw LC-MS/MS data was performed using MaxQuant (version 1.5.3.30) [50], and Andromeda search engine [51] was used for database searching against the UniProtKB/Swiss-Prot mouse database containing forward and reversed sequences (Mus musculus, Last modified: January 2018, Sequences: 74 228). MaxQuant also contains common contaminates proteins identified in proteomics analysis. MaxQuant analysis included an initial 
search with a precursor mass tolerance of $20 \mathrm{ppm}$, a main search precursor mass tolerance of 6 ppm and a fragment mass tolerance of $0.5 \mathrm{Da}$, respectively. Trypsin was selected as an enzyme, together with variable modifications such as methionine and proline oxidation, lysine acetylation, deamidation on asparagine or glutamine (NQ), serine, threonine phosphorylation and with the fixed modification carbamidomethyl cysteine. The minimal peptide length was set to six amino acids and the maximum number of missed cleavages to three. The matchbetween-runs function was used (match time window $=2 \mathrm{~min}$, alignment time window $=20$ min). The False Discovery Rate (FDR) was set to 0.01 for both peptide and protein identifications. The proteins identified by the completely same sets of peptides were grouped and reported as one protein group. Statistical analysis was performed with Perseus (1.6.0.2) [52]. The data were filtered to remove proteins "only identified by site", "reverse" sequence and "contaminant". The intensities of label free quantification (LFQ) values were transformed to $\log (2)$, then the data were filtered based on three valid values in at least one group (Figure $2-1.4)$. Missing values were imputed using random values derived from a distribution of $\log (2)-$ transformed protein values from samples. The distribution for values was created from the default settings (mean down shift $=0.3$, standard deviation down shift $=1.8$ ). Series of volcano plots were produced with $t$-test between two consecutive samples to illustrate significantly differentially abundant proteins (Figure 2-1.5). Principal Component Analyses (PCA) from ANOVA data were made with Perseus software. Both $t$-tests and one-way ANOVA tests were performed on $\log (2)$ data using a p-value of 0.01 and significance was determined using permutation-based FDR correction with standard parameters (FDR 0.01). The values obtained were then analyzed using Morpheus using the LFQ data (z-score) and Pearson hierarchical structuring (Figure 2-1.6), and STRING software (with mouse Mus Musculus database). Characterization of single protein expression was performed on the most up- and downregulated proteins, by introducing a variation threshold (fold change $|\mathrm{Fc}|>2$ ). 


\section{ReSUltS}

\subsection{BIOLOGICAL SAMPLE CHARACTERIZATION}

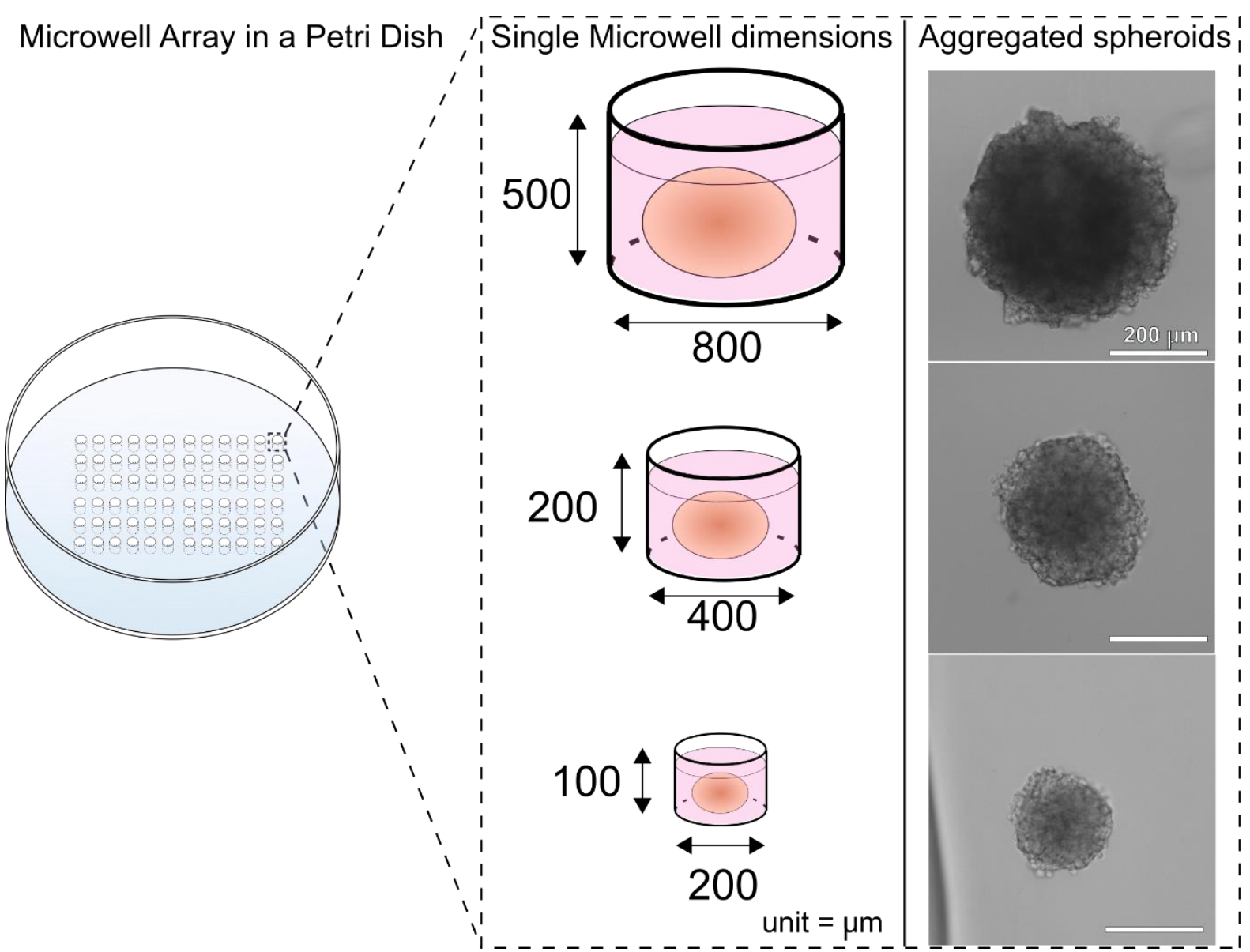

Figure 2-9 Multicellular tumor spheroid preparation. The spheroids were prepared from 4 T1 mouse breast cancer cells, in microwell arrays. Three sizes of microwells were employed: $800 \mu \mathrm{m}$ in diameter and $500 \mu \mathrm{m}$ in depth, $400 \mu \mathrm{m}$ and $200 \mu \mathrm{m}$ in depth, $200 \mu \mathrm{m}$ in diameter and $100 \mathrm{in}$ depth, $200 \mu \mathrm{m}$ in diameter and $100 \mu \mathrm{m}$ in depth. The cells inside the microwells aggregated into spheroids of "small", "medium", and "large" dimensions, and were collected after 5 days of incubation $\left(37^{\circ} \mathrm{C}, 5 \% \mathrm{CO}_{2}\right)$.

To prepare spheroids of three distinct sizes, microwell arrays of 200, 400 and $800 \mu \mathrm{m}$ in diameter were prepared, in which cells could aggregate (Figure 2-2). The reproducibility in size of resulting MCTS using this approach was assessed using standard microscopy. When first observing the MCTS in the microwell array, the spheroids top 2D projections appeared circular in shape, with an average apparent diameter of $205 \pm 8 \mu \mathrm{m}$ for the small MCTS, 274 $\pm 28 \mu \mathrm{m}$ for the medium MCTS, and $343 \pm 33 \mu \mathrm{m}$ for the large MCTS (Number of spheroids considered $n=6$ ). When imaging the MCTS trapped in agarose cubes, the spheroids side 2D projections presented an elliptical shape, indicating a small flattening of the spheroid, resulting 
in a height of $187 \pm 19$ (or 9\% flattening) for the small MCTS, $229 \pm 20 \mu \mathrm{m}$ (or $16 \%$ flattening) for the medium spheroid and $310 \pm 26 \mu \mathrm{m}$ (or 9\% flattening) for the large MCTS. This small flattening resulted in prolate-shaped spheroids, which potentially led to anisotropic gradients of nutrients and oxygen.

A wide variety of methods have been developed to generate arrays of MCTS of different sizes, shapes, and cellular compositions [53], and interestingly, depending on the cell origin, the aggregates reach different stable sizes, with varying final densities. Here, while the small MCTS presented sizes comparable to the microwells they aggregated in, the large MCTS reached diameters considerably smaller than the targeted size of $800 \mu \mathrm{m}$. Instead, small aggregates were found in suspension in the microwell array, as a result of cell shedding from the large MCTS. In a previous study, Gunther et al. characterized the evolution in size of mouse 4T1 MCTS over time, demonstrating that the aggregates reached a critical diameter size of $c a$. $450 \mu \mathrm{m}$ after one week in culture, accompanied by intense cell shedding, and subsequent smaller aggregate formation [54]. The observations in this study suggest that the microwell array method used here to generate MCTS was not responsible for the limitation in spheroid size formation, and that it would be impossible to achieve MCTS with diameter significantly larger than the ones presented here using this specific 4T1 breast mouse cell line.

\subsection{PRoteIn AND PEPTIDE QUANTIFICATION}

To investigate the relevance of the 3D MCTS as a model for cancer research, we analyzed the proteomic content from the different biological samples previously obtained, by first identifying their commonly expressed proteins, to then identify which among them varied between conditions.

\subsubsection{Proteome identification}

In this study, we chose to use the label-free quantification method to compare the different samples, as it is allows covering a wider range of the sample proteome compared to label approaches, while ignoring the need for cumbersome labeling workflow. Using nanoLCMS/MS, ca. 9000 peptides were identified in each condition (monolayer, spheroids small, medium, large), out of which $c a .7800$ were each unique to a single protein (Table 1). From these peptides, $c a$. 1632 proteins were identified in each condition, and after Perseus filtering to remove false positive measurements, 1245 (roughly 80\%) were present in all four conditions. 
The quality of the proteomic analysis was verified using the Pearson correlation of the quantified protein intensities among the replicates for each condition. The two biological samples and their three technical replicates were highly reproducible with coefficient of determination $R^{2}>0.90$, indicating a high correlation (Figure 2-S1). Furthermore, the protein intensity histograms presented an approximate log-normal distribution in each condition (Figure 2-S2), as required to apply null hypothesis for statistical analysis (i.e., student's $t$-test and ANOVA). Among the 1245 common proteins, 5 were identified with 1 peptide, 50 with 2 peptides and 1190 with more than 2 peptides, indicating high confidence in protein identification.

Table 2-1 Number of peptides, unique peptides and associated proteins found between all replicates for the different sample conditions (Monolayer and MCTS small, medium, large). The "number of common proteins in replicates" presents the number of proteins found in both biological replicates for each condition.

\begin{tabular}{|l|cccc|}
\cline { 2 - 5 } \multicolumn{1}{c|}{} & Monolayer & \multicolumn{3}{c|}{ MCTS } \\
\hline Number of peptides & 9466 & 9070 & 8716 & 9705 \\
$\begin{array}{l}\text { Number of unique } \\
\text { peptides }\end{array}$ & 7992 & 7680 & 7316 & 8257 \\
Number of proteins & 1621 & 1630 & 1602 & 1675 \\
$\begin{array}{l}\text { Number of common } \\
\text { proteins in replicates }\end{array}$ & 1351 & 1344 & 1318 & 1425 \\
\hline
\end{tabular}

The expression level of the 1245 common proteins was plotted as a heatmap to assess the differences between biological and analytical triplicates (Figure 2-S3). Due to the high reproducibility of results between analytical replicates, further analysis was performed on the average expression of these replicates in each biological condition, plotted as a heatmap in Figure 2-3B. Already, the hierarchical clustering of this average heat map presented, as expected, a closer similarity between all MCTS sizes against the monolayer.

Next, an ANOVA analysis of the 1245 common proteins identified 491 proteins whose expression levels varied between all conditions. All of the quantified 491 proteins were used for principal component analysis (PCA) using Perseus (Figure 2-3A). MCTS and monolayers 
clustered distinctly in Component 1 (45.2\%) and Component 2 (19.9\%) of the principal component space. PCA analysis resulted in the strict separation of spheroid samples and 2D monolayer cultures. However, between the MCTS of different sizes, the protein expression profiles of the small and medium spheroids presented great similarities, and were both significantly different from the large spheroids, possibly indicating a threshold in size above which the properties of the MCTS fundamentally changes.

\subsubsection{Protein up- and down-regulation in the MCTS}

To identify the proteins that changed significantly between the different conditions, one-way $t$-tests (FDR value $<0.01$ ) were performed on the 1245 proteins found common to all samples (Figure 2-S4). When comparing the different MCTS sizes to the monolayer, 304 proteins showed a different expression for the small spheroid models, 315 for the medium spheroid models, and 296 proteins for the large spheroid models. Among all these proteins, 84 were found common to all up-regulated in all MCTS sizes, up-regulated between all spheroid conditions as compared to the monolayer, and 53 were found down-regulated.

Due to the high amount of over- and under- expressed proteins, and since the label-free quantification only provides relative comparison of protein expression level between samples, the analysis of specific up-regulated proteins was focused on the proteins presenting the highest variation, by selecting a 2-fold change threshold [41]. Table 2-S1,S2 and S3 present the complete list of proteins over- and under- expressed between the different conditions (2-fold change). Using this threshold, 65 proteins were found up-regulated between all conditions, among which $18(27.7 \%)$ coincided between all three spheroid conditions, whereas only 4 (7.4\%) out of 54 down-regulated proteins were common to all conditions. Interestingly, the number of up- and down-regulated proteins between spheroid conditions compared to the monoculture differed, suggesting a variability given by the size of the environment, and a possible structural re-arrangements and physiological changes which influenced the cells through different level of cell-cell communication. Also, as previously mentioned, an increase in MCTS size is expected to be accompanied with an increase in nutrient and oxygen depletion, affecting the cell metabolism [55]. According to literature, this gradient becomes apparent in MCTS with diameters of $200 \mu \mathrm{m}$ or above [56]. The MCTS samples investigated in this article 
presented sizes of $c a$. 200, 275 and $350 \mu \mathrm{m}$ in diameter, and therefore it could be expected to observe a variation in expression of hypoxia-related pathways.
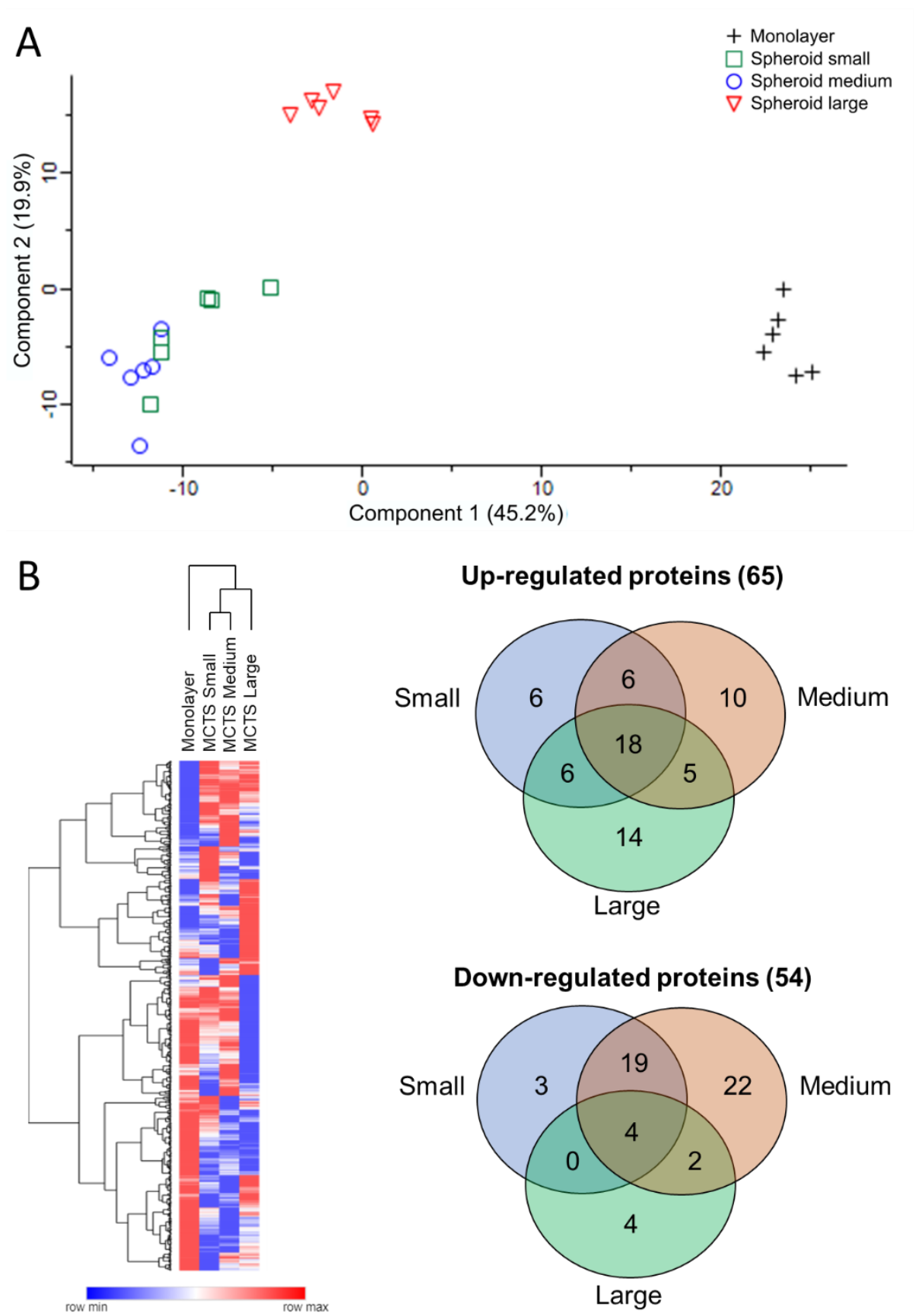

Figure 2-3 Proteomic analysis between the monolayer and the MCTS samples. A) Principal component analysis (PCA) of monolayers and spheroids proteomics data (z-score normalization). The PCA plot represent 491 proteins with significative difference after ANOVA test $(F D R=0.01)$ with biological and technical replicate that indicated clear proteomics profile difference between conditions. B) On the left, Morpheus generated heat map of the (average) protein expression profiles comparing the cancer monolayer and the three different sizes of spheroids. On the right, Venn diagram of the up-regulated and downregulated (2-fold change treshold) proteins for the 3D spheroid models compared to the $2 D$ model. 
As discussed in the introduction, several studies have previously been performed to characterize the impact of transitioning from $2 \mathrm{D}$ to $3 \mathrm{D}$ in vitro models on the protein expression profile and the resulting biological changes [36-45]. Mainly, they indicated an increase in metabolic activity in the MCTS. The cells in 3D in hypoxic region required more energy to maintain cellular functions, which as a result possibly decreased their ability to reproduce [57]. Additionally, regulation of stress was triggered as a result of 3D cellular reorganization. Depending on the cancer investigated, these studies also indicated the activation of specific cancer-associated pathways (e.g., immune escape, hypoxia) not found in monolayers, bridging between the gap between $2 \mathrm{D}$ in vitro models and the in vivo tumour formation. However, while in 2D monolayer the number of cell-cell interactions, and the access to nutrients and oxygen is uniform in the sample, cells in the 3D MCTS are potential exposed to a wide variety of stresses throughout the spheroid, suggesting that the size of the MCTS could impact the results on protein expression profiles. Here, beyond analyzing the comparison in protein expression profiles between $2 \mathrm{D}$ and $3 \mathrm{D}$ in vitro breast tumour models, a characterization of the impact of the MCTS size was therefore performed.

\subsection{FROM 2D TO 3D IN VITRO MODELS}

As a next step, to observe the variations of protein expression profiles between the $2 \mathrm{D}$ and 3D models, the 84 up-regulated and 53 down-regulated proteins commonly present in all MCTS models, as compared to the monolayer, were subjected to Gene Ontology (GO) and KEGG pathway analysis, and grouped by cellular component, biological process and molecular function, using STRING software.

\subsubsection{Up-regulated proteins in the MCTS vs. the monolayer}

When analyzing the 84 up-regulated proteins in all MCTS, several identified signaling pathways commonly established to be predominantly up-regulated in mouse and human breast cancer 3D in vitro MCTS models. Mainly, these pathways were: glycolysis, metabolic pathways, and biosynthesis of amino acids (Figure 2-S5) [58-60]. Additionally, HIF-1 $\alpha$ signaling pathway was also found up-regulated in the MCTS, indicating, as expected, the formation of hypoxic regions in all MCTS, even in the smallest spheroids.

When looking at the interaction of the 18 most up-regulated proteins (2-fold change, Figure 2-4), 3 are involved in the cytoskeleton reorganization (Tes, Tmsb4x, Crip1), indicating 
first that the aggregation of the cells into spheroids led to a structural modification of the cells as already reported in literature [61-63]; and second that, after 5 days in culture, the microtissues were still experiencing architectural modifications. Indeed, studies have previously shown gene and protein variations as a result of continuous adaptations of the cells to the environment [64]. As previously mentioned, the results presented here only describe the protein expression profiles at a single time-point (5th day of culture). It would be of a great interest to consider multiple time points of acquisition, to ascertain whether the protein expression profiles of the different spheroid conditions converge over time.

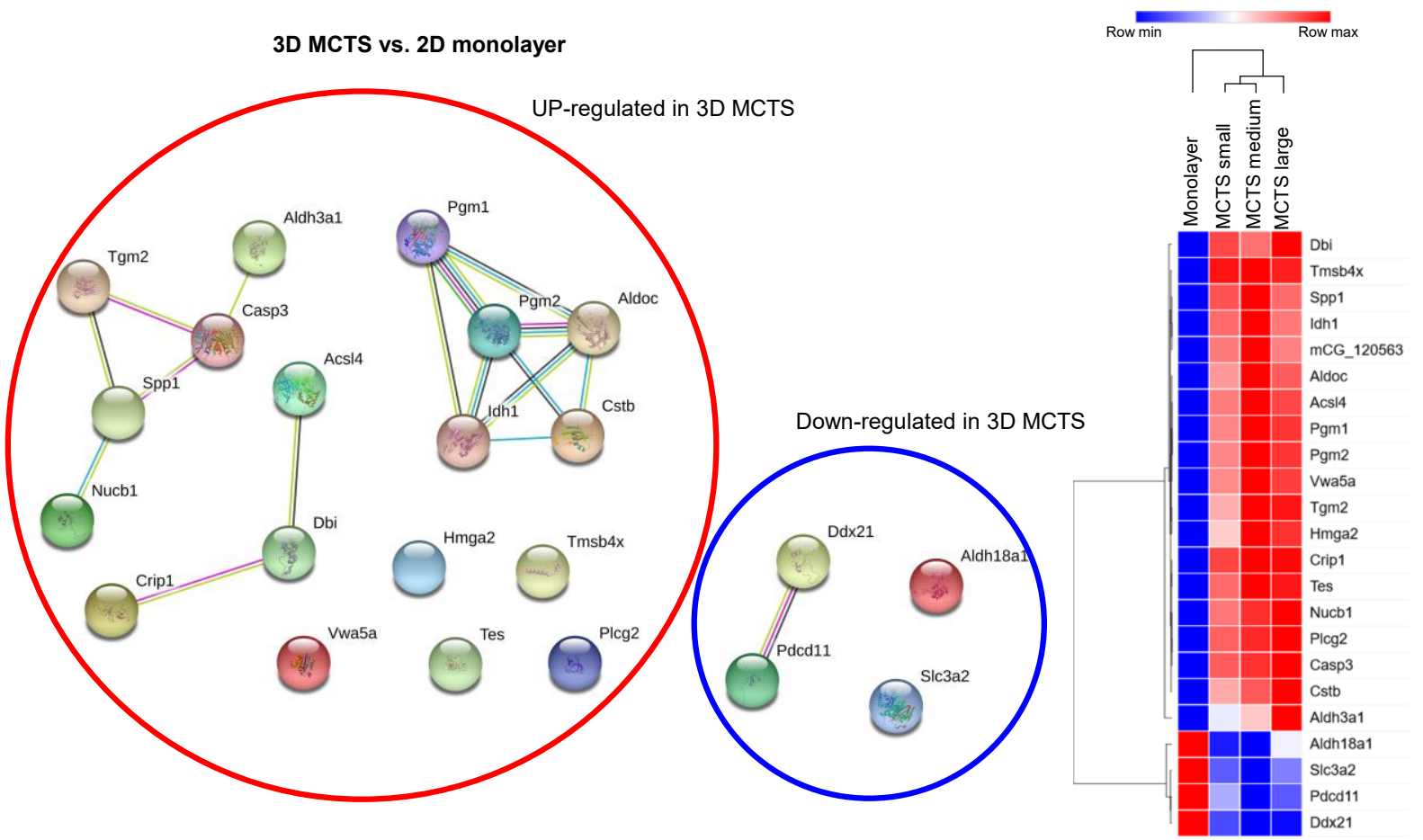

Figure 2-4 Up-and down-regulated proteins after 2-fold change threshold: $2 D$ vs 3D. On the left, proteins and their associations, found over-expressed (red circle) or under-expressed (blue circle) when comparing all three 3D tumour spheroids sizes expression against the monolayers, using STRING. On the right, Morpheus-generated heat map of the over and under-expressed proteins between all conditions.

A literature research was performed to assess the involvement of these proteins in cancer progression. Among the 18 up-regulated proteins, 11 (Crip1, Nucb1, Acs14, (Pgm1; Pgm2), Tmsb4x, Spp1, Aldh3a1, Casp3, Tgm2, Hmga2, Cstb) were all proteins found to be highly expressed in cancers [65-75]. (Table 2-S2). Among those, 7 (Acs14, Tmsb4x, Spp1, Casp3, Tgm2, Hmga2, Crip1) showed to be up-regulated in breast cancer studies [69-75]. This already concurs with the established notion that 3D models are more representative models than monolayers. 
Interestingly, Caspase-3, one of the key-executioners of apoptosis was up-regulated in all three spheroid conditions. This would suggest a strong occurrence of cell death due to the formation of an apoptotic core within the spheroids. However, the maximum size of the spheroids obtained here $(\mathrm{ca} .345 \mu \mathrm{m})$ is in theory insufficient to present a nutrient-deprived core and other proteins related to this cell death pathway were not found up-regulated [56]. During the caspase cascade, each caspase undergoes a cleavage which triggers the activation of the following caspase molecule and consequently leads to cell debris formation and death. This would suggest that the Caspase-3 measured here is not cleaved. Moreover, Caspase-3 has shown a strong contribution in cell growth in breast cancer tumours, and several studies have been focused on activating or de-activating this pathway to enhance tumour cell death activation $[71,76]$.

Among the 18 up-regulated proteins, Testin LIM domain protein (Tes), normally involved in cell motility and cell adhesion, has been reported down-regulated in in vivo human cancer studies $[62,77,78]$ and has been targeted as a potential tumour suppressor in breast cancer [62, 78], [79]. The disparity of expression levels could be explained here by the involvement of Tes protein in the cytoskeleton reorganization. Unlike tumours slowly growing from a single cancer cell, the rapid aggregation of cells into spheroids may have resulted in the overexpression of proteins involved in the process of cell-cell interaction. This hypothesis could be verified by testing the expression level of Tes in the MCTS after longer periods of incubation, where for instance the small MCTS have grown to match in size the large MCTS.

\subsubsection{Down-regulated proteins in the MCTS vs. the monolayer}

Among the proteins down-regulated in the spheroids against the monolayers, 53 were identified in all spheroid conditions. The GO enrichment of these proteins indicated in great majority the down-regulation of pathways involved in RNA processing and cellular component organization, phenomena also commonly observed in cancer [80]. However, when focusing on the most under-expressed proteins (2-fold change), 3 have shown to be overexpressed in human breast cancer studies in vivo (Slc3a2, Ddx21, Aldh18a1) [81-83].

The 4F2 cell-surface antigen heavy chain (Slc3a2) is part of a solute carrier family which has been shown to play a role in human breast cancer growth [83, 84]. This protein enhances the integrin signals increasing the remodeling and promotes cancer proliferation [83]. This discrepancy in protein expression level with our results could be related to the difference in size of the spheroid models. Our study makes use of microtissues of up to $350 \mu \mathrm{m}$ in diameter 
whereas in the study of Rokaya El Ansari [83] the samples are tumour ex vivo sections of 0.6 $\mathrm{cm}$ in size minimum. Moreover, our study uses a Mus musculus model whereas El Ansari et al. use patient samples.

DEAD-box RNA 21 (DDx21) is part of the 59 DEAD/H-box helicase family. This cluster of proteins is multifunctional and participate to a range of biological processes involved in micro-RNA manipulation [85]. These DEAD/H-box proteins have shown to have regulatory effects on several cellular signaling pathways involved in tumour progression, and are investigated as potential biomarkers for cancer detection [85]. Especially, the up-regulation of DDx21 has shown to enhance tumorigenesis in human ex vivo breast tissues by intervening in the cellular differentiation of the proto-oncogene c-Jun [86]. Since the cells rapidly aggregate into MCTS inside the microwell array, it would be of interest to determine whether the downregulation in expression of DDx21 is time- or structure-regulated, by measuring its activity in MCTS of different sizes during the first days of spheroid formation.

Aldh18a1 belongs to the family of aldehyde dehydrogenases (ALDH), which are critical enzymes for the detoxification of aldehyde substrates. These proteins, especially Aldh1a1 and Aldh3a1, have shown to enhance cell survival against cytotoxic drugs [87]. However, little is known about Aldh18a1 role in the tumour progression. Interestingly, the expression level of Aldh18a1 here increases with the MCTS sizes, suggesting a correlation with the tumour size. Therefore, limiting the proteomic analysis to $2 \mathrm{D}$ against $3 \mathrm{D}$ models is insufficient to characterize the relevance of the MCTS as a cancer in vitro model.

\subsection{FROM SMALL TO LARGE SPHEROIDS}

In a final step, after having established the variations in protein expression profiles between the monolayers and the spheroids, the impact of the MCTS size on the activation of specific signaling pathways, as well as the variations of protein expression profiles between the three different MCTS samples (small, medium, large), were analyzed using STRING

software. When comparing the proteomic expression profiles between the different spheroid sizes, 4 proteins were found statistically different between the medium and small spheroids, 125 between the large and small spheroids, and 144 between the large and medium spheroids.

First, a consistent increase in metabolic processes was detected in correlation with the increase in size of the MCTS. Then, when comparing the large MCTS to the small and medium 
ones, an increase in oxidoreductase activity (Aldh3a1, Aldh18a1, Cbr2) was observed in in the large MCTS, translating to an increase in oxygen consumption of the MCTS [88]. However, studies have shown a decrease in oxygen consumption as a result of tumour increase in size and density leading to hypoxic regions where the glycolysis process switch from aerobic to anaerobic, and only cells in proximity of an oxygen source (i.e., blood vessels) could undergo the oxydo-reduction process [89]. The increase in oxygen consumption here could be resulting from a difference in the energy required to perform structural modifications (i.e., cytoskeleton reorganization, oxidative stress) when generating the MCTS.

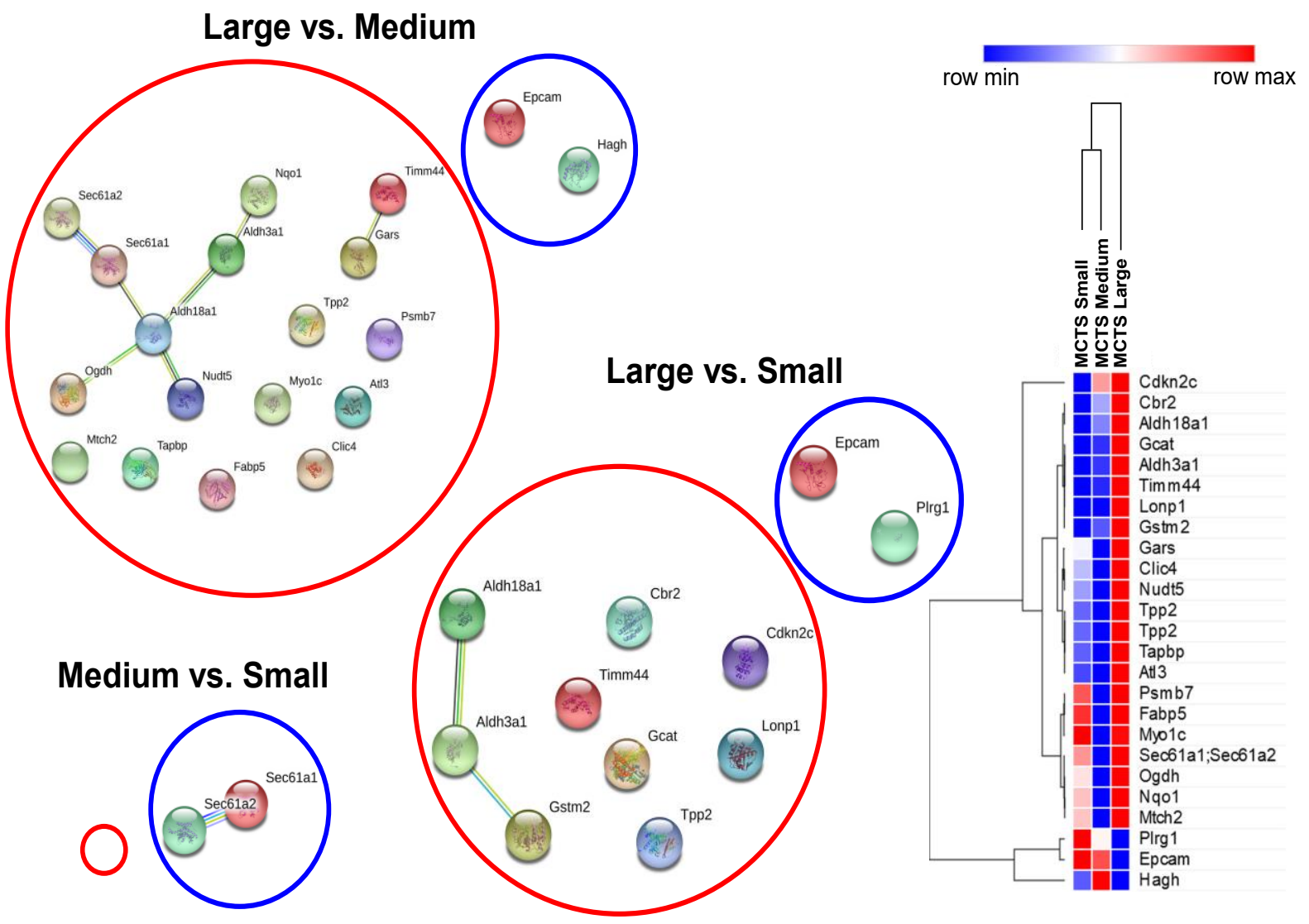

Figure 2-5: Comparison of protein expression profiles between spheroids of different sizes. On the left, lists of genes and their interactions, associated with the proteins found over-expressed (red circles) or under-expressed (blue circles) when comparing the spheroids of different sizes (small, medium, large) (2-fold change threshold). On the right, Morpheus generated heat map of the over-and under-expressed proteins when comparing the different spheroid sizes.

On the other hand, when increasing the size of the MCTS, a de-activation of the cell motility pathways was observed. Cell motility in cancer cells has shown to contribute to tumour metastasis [90], which could indicate a separation of the metastatic elements (circulating tumour cells) present in the tumour spheroid. 
After applying the 2-fold change threshold, the most significant variations were detected by comparing the large and medium spheroids, where 17 proteins were found upregulated in the large spheroids and only 2 were found down-regulated (Figure 2-5). This contrast was reduced when comparing the large to the small spheroid ( 9 up-regulated \& 2 down-regulated proteins in the large MCTS) and was approximately inexistent between medium and small spheroids (no upregulated proteins and only 2 down-regulated ones in the medium). This result suggests, as previously observed in the principal component analysis (Figure 2-3.a), the existence of a threshold between the medium and large MCTS, even though the MCTS diameter difference between both conditions is of $c a .75 \mu \mathrm{m}$.

A literature analysis, performed on the 24 proteins presenting significant variations between the different spheroid sizes, identified 16 proteins labeled as key components in the regulation of different cancers (e.g., colon, lung, prostate, pancreas). Additionally, 5 of these proteins were found to be over-expressed in human 2D in vitro (Nudt5, Tpp2) or in vivo (EpCAM, Fabp5, Nqo1) breast cancer studies [91-95]. Furthermore, Nqo1 is considered as a good biomarker for the prognostic of breast cancer [93].

When comparing the large spheroids to both the medium and small spheroids, 4 proteins (Timm44, Aldh18a1, Aldh3a1, Tpp2) were up-regulated and one (EpCAM) was down-regulated in the large MCTS. Timm44 is a mitochondrial inner translocase protein, and although no study has determined its involvement in breast cancer progression, it has been found up-regulated in thyroid both in in vitro and ex vivo in human samples [96]. Aldh18a1 and Aldh3a1, part of the aldehyde dehydrogenase (ALDH) family, have, as previously mentioned, shown enhanced survival potential for tumour cells [87]. Finally, Tripeptidylpeptidase 2 (Tpp2) is an enzyme involved in a wide variety of molecular functions, including DNA repair and cell apoptosis. Tpp2 has been reported to participate in the mitotic exit of malignant cells, promoting the cell division and therefore tumour proliferation [94]. Altogether, these four up-regulated proteins have shown involvement in cancer (e.g., thyroid, heart, kidney), but further investigation is required to assess their involvement in breast cancer.

EpCAM, a transmembrane protein normally involved in the mediation cell-cell adhesion and identified as a breast cancer epithelial marker [97], was found down-regulated in the large spheroids compared to both the small and medium ones. The down-regulation of EpCAM in the large spheroids could be explained by the increase in MCTS size leading to a proportional increase of cells inside the spheroid which undergo an epithelial-mesenchymal 
transition (EMT). Through this process the cells lose their polarity and cell-cell adhesion, promoting the metastatic process [98]. Previous studies have shown that this EMT was promoted in cancer by the presence of hypoxia and tumour induced growth factors [99].

The variations in protein expression profiles of the three conditions (small, medium and large MCTS) suggests a clear influence of the size of the MCTS on their behaviors. This would imply that not only patient variability could affect drug response [100], but also, as already known that depending on the size of the tumour, the cancer cells could present a different response to treatments.

The proteomic analyses performed in this study showed that already on tumour spheroids composed of a single cell type (i.e., cancer cells), the smallest variation in size affected the protein expression profile. In addition, previous study have shown that between donors, the protein expression profile as well as the response to treatment of cancer cells varied [100]. This implies that to reliably compare the results between samples and studies, the methods of preparation and culture of the MCTS needs to be standardized, as previously investigated by Froehlich et al. [101].

\section{CONClusion}

As also previously presented in the literature, this study demonstrates how, in cancer research, transitioning from a standard 2D monolayer model to a more in vivo like 3D model significantly affects the protein expression profile. Especially a significant number of proteins has previously been found to be affected in in vivo breast tumour development, indicating that the model presented here is better suited to perform breast cancer biological assays. Also, structure plays an important role in cell protein synthesis. As a result, the 3D MCTS is a better model to study the presence of up- or down-regulated proteins, the activation of specific signaling pathways, and to investigate the response to drug treatment. The MCTS presented in this study however showed structural changes after 5 days, indicating that the MCTS still hadn't reach their critical size, which fuels the need for a characterization study of the protein expression profiles over time. 
Then, despite presenting MCTS with only small incrementation in size, an increase in activated hypoxia-related pathways was observed, accompanied with a decrease in cell motility pathways related to metastasis processes. Because the MCTS in this study could not grow beyond a certain size, the further evolution of this mechanism could not be investigated. Moreover, a significant number of proteins involved in the cancer progression were up- or down- regulated between the three different MCTS sizes, revealing the need for proper MCTS characterization, for instance prior to targeted therapeutic assays.

Finally, as discussed in the introduction and throughout this thesis, the actual tumour environment possesses a higher degree in complexity, having both vascularization and presenting multiple types of cells in the tissue. To further improve the 3D MCTS model as an alternative to the current monolayer platform, it would be of great interest to integrate other cells present in the tumour microenvironment (e.g., fibroblasts, macrophages) in the MCTS, and perform the same proteomic analysis.

\section{ACKNOWLEDGMENT}

This work was performed in collaboration with Prof. Christian Rolando and Dr. Fabrice Bray from the University of Lille, France. It was also strongly supported by the active participation of PhD candidate Carlo Alberto Paggi from the Developmental BioEngineering group at the University of Twente.

\section{REFERENCES}

1. Karunaratne, D.N., Silverstein, P. S., Vasandani, V., Young, A. M., Rytting, E., Yops, B., \& Audus, K. L., Cell Culture Models for Drug Transport Studies, in Drug Delivery: Principles and Applications. 2005, John Wiley \& Sons, Inc. p. 103-124.

2. Breslin, S. and L. O'Driscoll, Three-dimensional cell culture: the missing link in drug discovery. Drug Discov Today, 2013. 18(5-6): p. 240-9.

3. Fitzgerald, K.A., et al., Life in $3 D$ is never flat: $3 D$ models to optimise drug delivery. J Control Release, 2015. 215: p. 39-54.

4. Monteiro, M.V., et al., Hydrogel 3D in vitro tumor models for screening cell aggregation mediated drug response. Biomater Sci, 2020. 8(7): p. 1855-1864.

5. Sutherland, R.M., Cell and environment interactions in tumor microregions: the multicell spheroid model. Science, 1988. 240(4849): p. 177-84.

6. Mehta, G., et al., Opportunities and challenges for use of tumor spheroids as models to test drug delivery and efficacy. J Control Release, 2012. 164(2): p. 192-204. 
7. Nath, S. and G.R. Devi, Three-dimensional culture systems in cancer research: Focus on tumor spheroid model. Pharmacol Ther, 2016. 163: p. 94-108.

8. Daster, S., et al., Induction of hypoxia and necrosis in multicellular tumor spheroids is associated with resistance to chemotherapy treatment. Oncotarget, 2017. 8(1): p. 1725-1736.

9. Tung, Y.C., et al., High-throughput 3D spheroid culture and drug testing using a 384 hanging drop array. Analyst, 2011. 136(3): p. 473-8.

10. Tredan, O., et al., Drug resistance and the solid tumor microenvironment. J Natl Cancer Inst, 2007. 99(19): p. 1441-54.

11. Horsman, M.R. and J. Overgaard, The impact of hypoxia and its modification of the outcome of radiotherapy. J Radiat Res, 2016. 57 Suppl 1: p. i90-i98.

12. Cui, X., Y. Hartanto, and H. Zhang, Advances in multicellular spheroids formation. J R Soc Interface, 2017. 14(127).

13. Picollet-D'hahan, N., et al., A 3D Toolbox to Enhance Physiological Relevance of Human Tissue Models. Trends Biotechnol, 2016. 34(9): p. 757-769.

14. Fennema, E., et al., Spheroid culture as a tool for creating 3D complex tissues. Trends Biotechnol, 2013. 31(2): p. 108-15.

15. Sridhar, A., et al., Microstamped Petri dishes for scanning electrochemical microscopy analysis of arrays of microtissues. PLoS One, 2014. 9(4): p. e93618.

16. Hardelauf, H., et al., Microarrays for the scalable production of metabolically relevant tumour spheroids: a tool for modulating chemosensitivity traits. Lab Chip, 2011. 11(3): p. 419-28.

17. Torisawa, Y.S., et al., Efficient formation of uniform-sized embryoid bodies using a compartmentalized microchannel device. Lab Chip, 2007. 7(6): p. 770-6.

18. Chan, H.F., et al., Rapid formation of multicellular spheroids in double-emulsion droplets with controllable microenvironment. Sci Rep, 2013. 3: p. 3462.

19. Sabhachandani, P., et al., Generation and functional assessment of 3D multicellular spheroids in droplet based microfluidics platform. Lab Chip, 2016. 16(3): p. 497-505.

20. Sart, S., et al., Mapping the structure and biological functions within mesenchymal bodies using microfluidics. Sci Adv, 2020. 6(10): p. eaaw7853.

21. Tomasi, R.F., et al., Individual Control and Quantification of 3D Spheroids in a HighDensity Microfluidic Droplet Array. Cell Rep, 2020. 31(8): p. 107670.

22. Yaman, S., et al., Magnetic Force-Based Microfluidic Techniques for Cellular and Tissue Bioengineering. Front Bioeng Biotechnol, 2018. 6: p. 192.

23. Chen, K., et al., Rapid formation of size-controllable multicellular spheroids via 3D acoustic tweezers. Lab Chip, 2016. 16(14): p. 2636-43.

24. Sebastian, A., A.M. Buckle, and G.H. Markx, Tissue engineering with electric fields: immobilization of mammalian cells in multilayer aggregates using dielectrophoresis. Biotechnol Bioeng, 2007. 98(3): p. 694-700.

25. Wrzesinski, K. and S.J. Fey, Metabolic Reprogramming and the Recovery of Physiological Functionality in 3D Cultures in Micro-Bioreactors. Bioengineering (Basel), 2018. 5(1).

26. Wrzesinski, K. and S.J. Fey, From 2D to 3D--a New Dimension for Modelling the Effect of Natural Products on Human Tissue. Curr Pharm Des, 2015. 21(38): p. 5605-16.

27. Wrzesinski, K., et al., The cultural divide: exponential growth in classical $2 D$ and metabolic equilibrium in 3D environments. PLoS One, 2014. 9(9): p. e106973. 
28. Junttila, M.R. and F.J. de Sauvage, Influence of tumour micro-environment heterogeneity on therapeutic response. Nature, 2013. 501(7467): p. 346-54.

29. Netti, P.A., et al., Role of extracellular matrix assembly in interstitial transport in solid tumors. Cancer Res, 2000. 60(9): p. 2497-503.

30. Priwitaningrum, D.L., et al., Tumor stroma-containing 3D spheroid arrays: $A$ tool to study nanoparticle penetration. J Control Release, 2016. 244(Pt B): p. 257-268.

31. Birgersdotter, A., R. Sandberg, and I. Ernberg, Gene expression perturbation in vitro-a growing case for three-dimensional (3D) culture systems. Semin Cancer Biol, 2005. 15(5): p. 405-12.

32. Reynolds, D.S., et al., Breast Cancer Spheroids Reveal a Differential Cancer Stem Cell Response to Chemotherapeutic Treatment. Sci Rep, 2017. 7(1): p. 10382.

33. Isakov, N., Protein kinase C (PKC) isoforms in cancer, tumor promotion and tumor suppression. Semin Cancer Biol, 2018. 48: p. 36-52.

34. Krueger, K.E. and S. Srivastava, Posttranslational protein modifications: current implications for cancer detection, prevention, and therapeutics. Mol Cell Proteomics, 2006. 5(10): p. 1799-810.

35. Macfarlane, S.R., et al., Proteinase-activated receptors. Pharmacol Rev, 2001. 53(2): p. 245-82.

36. Poland, J., et al., Comparison of protein expression profiles between monolayer and spheroid cell culture of HT-29 cells revealed fragmentation of CK18 in threedimensional cell culture. Electrophoresis, 2002. 23(7-8): p. 1174-84.

37. Kumar, H.R., et al., Three-dimensional neuroblastoma cell culture: proteomic analysis between monolayer and multicellular tumor spheroids. Pediatr Surg Int, 2008. 24(11): p. 1229-34.

38. Gaedtke, L., et al., Proteomic analysis reveals differences in protein expression in spheroid versus monolayer cultures of low-passage colon carcinoma cells. J Proteome Res, 2007. 6(11): p. 4111-8.

39. Gebhard, C., et al., Comparative proteome analysis of monolayer and spheroid culture of canine osteosarcoma cells. J Proteomics, 2018. 177: p. 124-136.

40. Machida, M., et al., Proteomic comparison of spherical aggregates and adherent cells of cardiac stem cells. Int J Cardiol, 2011. 153(3): p. 296-305.

41. Yue, X., et al., Quantitative Proteomic and Phosphoproteomic Comparison of 2D and 3D Colon Cancer Cell Culture Models. J Proteome Res, 2016. 15(12): p. 4265-4276.

42. Tolle, R.C., C. Gaggioli, and J. Dengjel, Three-Dimensional Cell Culture Conditions Affect the Proteome of Cancer-Associated Fibroblasts. J Proteome Res, 2018. 17(8): p. 27802789.

43. Lee, S.Y., et al., iTRAQ-Based Quantitative Proteomic Comparison of $2 D$ and $3 D$ Adipocyte Cell Models Co-cultured with Macrophages Using Online 2D-nanoLC-ESIMS/MS. Sci Rep, 2019. 9(1): p. 16746.

44. He, W., et al., Proteomic comparison of 3D and 2D glioma models reveals increased HLA-E expression in 3D models is associated with resistance to NK cell-mediated cytotoxicity. J Proteome Res, 2014. 13(5): p. 2272-81.

45. McMahon, K.M., et al., Characterization of changes in the proteome in different regions of 3D multicell tumor spheroids. J Proteome Res, 2012. 11(5): p. 2863-75.

46. Ravindran Menon, D., et al., CDK1 Interacts with Sox2 and Promotes Tumor Initiation in Human Melanoma. Cancer Res, 2018. 78(23): p. 6561-6574. 
47. Lu, T., et al., Multi-omics profiling reveals key signaling pathways in ovarian cancer controlled by STAT3. Theranostics, 2019. 9(19): p. 5478-5496.

48. Feist, P.E., et al., Multicellular Tumor Spheroids Combined with Mass Spectrometric Histone Analysis To Evaluate Epigenetic Drugs. Anal Chem, 2017. 89(5): p. 2773-2781.

49. Erde, J., R.R. Loo, and J.A. Loo, Enhanced FASP (eFASP) to increase proteome coverage and sample recovery for quantitative proteomic experiments. J Proteome Res, 2014. 13(4): p. 1885-95.

50. Cox, J. and M. Mann, MaxQuant enables high peptide identification rates, individualized p.p.b.-range mass accuracies and proteome-wide protein quantification. Nat Biotechnol, 2008. 26(12): p. 1367-72.

51. Cox, J., et al., Andromeda: a peptide search engine integrated into the MaxQuant environment. J Proteome Res, 2011. 10(4): p. 1794-805.

52. Tyanova, S., et al., The Perseus computational platform for comprehensive analysis of (prote)omics data. Nat Methods, 2016. 13(9): p. 731-40.

53. Friedrich, J., R. Ebner, and L.A. Kunz-Schughart, Experimental anti-tumor therapy in 3D: spheroids--old hat or new challenge? Int J Radiat Biol, 2007. 83(11-12): p. 849-71.

54. Gunther, S., et al., Polyphenols prevent cell shedding from mouse mammary cancer spheroids and inhibit cancer cell invasion in confrontation cultures derived from embryonic stem cells. Cancer Lett, 2007. 250(1): p. 25-35.

55. Hirschhaeuser, F., U.G. Sattler, and W. Mueller-Klieser, Lactate: a metabolic key player in cancer. Cancer Res, 2011. 71(22): p. 6921-5.

56. Riffle, S., et al., Linking hypoxia, DNA damage and proliferation in multicellular tumor spheroids. BMC Cancer, 2017. 17(1): p. 338.

57. Hubbi, M.E. and G.L. Semenza, Regulation of cell proliferation by hypoxia-inducible factors. Am J Physiol Cell Physiol, 2015. 309(12): p. C775-82.

58. Vander Heiden, M.G., L.C. Cantley, and C.B. Thompson, Understanding the Warburg effect: the metabolic requirements of cell proliferation. Science, 2009. 324(5930): p. 1029-33.

59. Hunnewell, M.G. and N.S. Forbes, Active and inactive metabolic pathways in tumor spheroids: determination by GC-MS. Biotechnol Prog, 2010. 26(3): p. 789-96.

60. Rodriguez-Enriquez, S., et al., Energy metabolism transition in multi-cellular human tumor spheroids. J Cell Physiol, 2008. 216(1): p. 189-97.

61. Morita, T. and K. Hayashi, Tumor Progression Is Mediated by Thymosin-beta4 through a TGFbeta/MRTF Signaling Axis. Mol Cancer Res, 2018. 16(5): p. 880-893.

62. Wang, D.D., et al., TES functions as a Mena-dependent tumor suppressor in gastric cancer carcinogenesis and metastasis. Cancer Commun (Lond), 2019. 39(1): p. 3.

63. Zhang, L.Z., et al., CRIP1 promotes cell migration, invasion and epithelial-mesenchymal transition of cervical cancer by activating the Wnt/betacatenin signaling pathway. Life Sci, 2018. 207: p. 420-427.

64. Edmondson, R., et al., Three-dimensional cell culture systems and their applications in drug discovery and cell-based biosensors. Assay Drug Dev Technol, 2014. 12(4): p. 20718.

65. Bae, E., et al., Phosphoglucomutase1 is necessary for sustained cell growth under repetitive glucose depletion. FEBS Lett, 2014. 588(17): p. 3074-80.

66. Chen, Y., et al., Autoantibodies to $\mathrm{Ca} 2+$ binding protein Calnuc is a potential marker in colon cancer detection. Int J Oncol, 2007. 30(5): p. 1137-44. 
67. Lee, J.S., et al., Gastric cancer depends on aldehyde dehydrogenase $3 A 1$ for fatty acid oxidation. Sci Rep, 2019. 9(1): p. 16313.

68. Wang, X., et al., Cystatin B is a progression marker of human epithelial ovarian tumors mediated by the TGF-beta signaling pathway. Int J Oncol, 2014. 44(4): p. 1099-106.

69. Wu, J., et al., Elevated HMGA2 expression is associated with cancer aggressiveness and predicts poor outcome in breast cancer. Cancer Lett, 2016. 376(2): p. 284-92.

70. Agnihotri, N., S. Kumar, and K. Mehta, Tissue transglutaminase as a central mediator in inflammation-induced progression of breast cancer. Breast Cancer Res, 2013. 15(1): p. 202.

71. Olsson, M. and B. Zhivotovsky, Caspases and cancer. Cell Death Differ, 2011. 18(9): p. 1441-9.

72. Zhang, X., et al., Thymosin beta 10 is a key regulator of tumorigenesis and metastasis and a novel serum marker in breast cancer. Breast Cancer Res, 2017. 19(1): p. 15.

73. Sharon, Y., et al., Tumor-derived osteopontin reprograms normal mammary fibroblasts to promote inflammation and tumor growth in breast cancer. Cancer Res, 2015. 75(6): p. 963-73.

74. Ludyga, N., et al., The impact of cysteine-rich intestinal protein 1 (CRIP1) in human breast cancer. Mol Cancer, 2013. 12: p. 28.

75. Wu, X., et al., Long chain fatty Acyl-CoA synthetase 4 is a biomarker for and mediator of hormone resistance in human breast cancer. PLoS One, 2013. 8(10): p. e77060.

76. Huang, Q., et al., Caspase 3-mediated stimulation of tumor cell repopulation during cancer radiotherapy. Nat Med, 2011. 17(7): p. 860-6.

77. Garvalov, B.K., et al., The conformational state of Tes regulates its zyxin-dependent recruitment to focal adhesions. J Cell Biol, 2003. 161(1): p. 33-9.

78. Chene, L., et al., Extensive analysis of the $7 q 31$ region in human prostate tumors supports TES as the best candidate tumor suppressor gene. Int J Cancer, 2004. 111(5): p. 798-804.

79. Zhu, J., et al., Testin is a tumor suppressor and prognostic marker in breast cancer. Cancer Sci, 2012. 103(12): p. 2092-101.

80. Williams, M., et al., Exploring Mechanisms of MicroRNA Downregulation in Cancer. Microrna, 2017. 6(1): p. 2-16.

81. Zhang, $\mathrm{H}$., et al., A double-negative feedback loop between DEAD-box protein DDX21 and Snail regulates epithelial-mesenchymal transition and metastasis in breast cancer. Cancer Lett, 2018. 437: p. 67-78.

82. Grinde, M.T., et al., Glutamine to proline conversion is associated with response to glutaminase inhibition in breast cancer. Breast Cancer Res, 2019. 21(1): p. 61.

83. El Ansari, R., et al., The multifunctional solute carrier 3A2 (SLC3A2) confers a poor prognosis in the highly proliferative breast cancer subtypes. Br J Cancer, 2018. 118(8): p. 1115-1122.

84. Furuya, M., et al., Correlation of L-type amino acid transporter 1 and CD98 expression with triple negative breast cancer prognosis. Cancer Sci, 2012. 103(2): p. 382-9.

85. Cai, W., et al., Wanted DEAD/H or Alive: Helicases Winding Up in Cancers. J Natl Cancer Inst, 2017. 109(6).

86. Zhang, Y., et al., Elevated DDX21 regulates c-Jun activity and $r R N A$ processing in human breast cancers. Breast Cancer Res, 2014. 16(5): p. 449.

87. Tomita, H., et al., Aldehyde dehydrogenase $1 A 1$ in stem cells and cancer. Oncotarget, 2016. 7(10): p. 11018-32. 
88. Herst, P.M. and M.V. Berridge, Cell surface oxygen consumption: a major contributor to cellular oxygen consumption in glycolytic cancer cell lines. Biochim Biophys Acta, 2007. 1767(2): p. 170-7.

89. Vaupel, P., et al., Blood flow, oxygen consumption, and tissue oxygenation of human breast cancer xenografts in nude rats. Cancer Res, 1987. 47(13): p. 3496-503.

90. Stuelten, C.H., C.A. Parent, and D.J. Montell, Cell motility in cancer invasion and metastasis: insights from simple model organisms. Nat Rev Cancer, 2018. 18(5): p. 296-312.

91. Liu, R.Z., et al., Association of FABP5 expression with poor survival in triple-negative breast cancer: implication for retinoic acid therapy. Am J Pathol, 2011. 178(3): p. 9971008.

92. Osta, W.A., et al., EpCAM is overexpressed in breast cancer and is a potential target for breast cancer gene therapy. Cancer Res, 2004. 64(16): p. 5818-24.

93. Yang, Y., et al., Clinical implications of high NQO1 expression in breast cancers. J Exp Clin Cancer Res, 2014. 33: p. 14.

94. Ziegler, Y.S., et al., Proteomic analysis identifies highly expressed plasma membrane proteins for detection and therapeutic targeting of specific breast cancer subtypes. Clin Proteomics, 2018. 15: p. 30.

95. Page, B.D.G., et al., Targeted NUDT5 inhibitors block hormone signaling in breast cancer cells. Nat Commun, 2018. 9(1): p. 250.

96. Wang, M.H., et al., Epithelial cell adhesion molecule overexpression regulates epithelial-mesenchymal transition, stemness and metastasis of nasopharyngeal carcinoma cells via the PTEN/AKT/mTOR pathway. Cell Death Dis, 2018. 9(1): p. 2.

97. Gostner, J.M., et al., Effects of EpCAM overexpression on human breast cancer cell lines. BMC Cancer, 2011. 11: p. 45.

98. Mittal, V., Epithelial Mesenchymal Transition in Tumor Metastasis. Annu Rev Pathol, 2018. 13: p. 395-412.

99. Roche, J., The Epithelial-to-Mesenchymal Transition in Cancer. Cancers (Basel), 2018. 10(2).

100. Yang, R., et al., Dissecting variability in responses to cancer chemotherapy through systems pharmacology. Clin Pharmacol Ther, 2010. 88(1): p. 34-8.

101. Froehlich, K., et al., Generation of Multicellular Breast Cancer Tumor Spheroids: Comparison of Different Protocols. J Mammary Gland Biol Neoplasia, 2016. 21(3-4): p. 89-98. 


\section{APPENDIX}
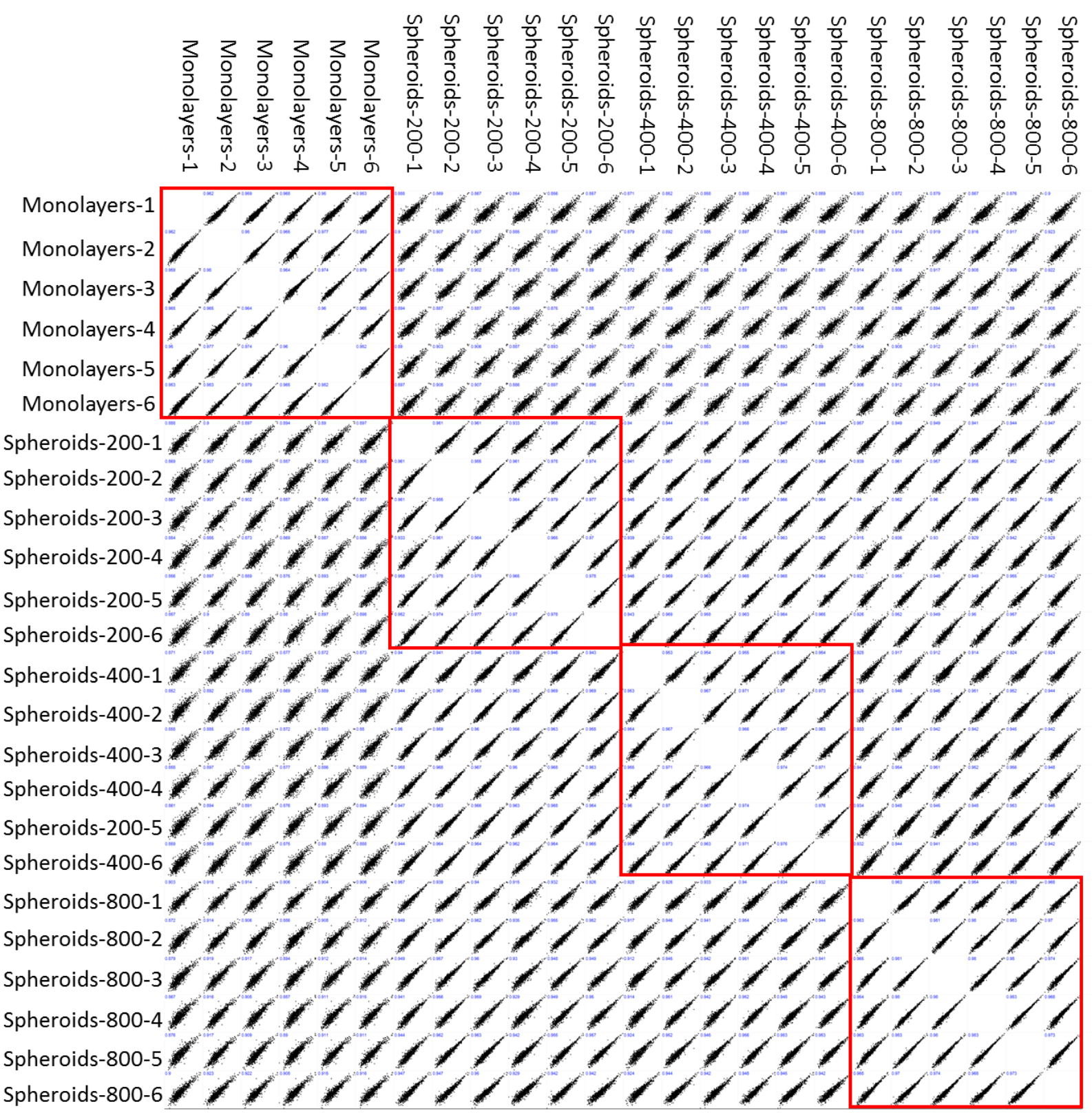

Figure 2-S1 Person correlation of log2 data from proteomics analysis. Technical reproducibility was confirmed with high Pearson correlation $(\geq 0.90)$ between the replicate, red square 

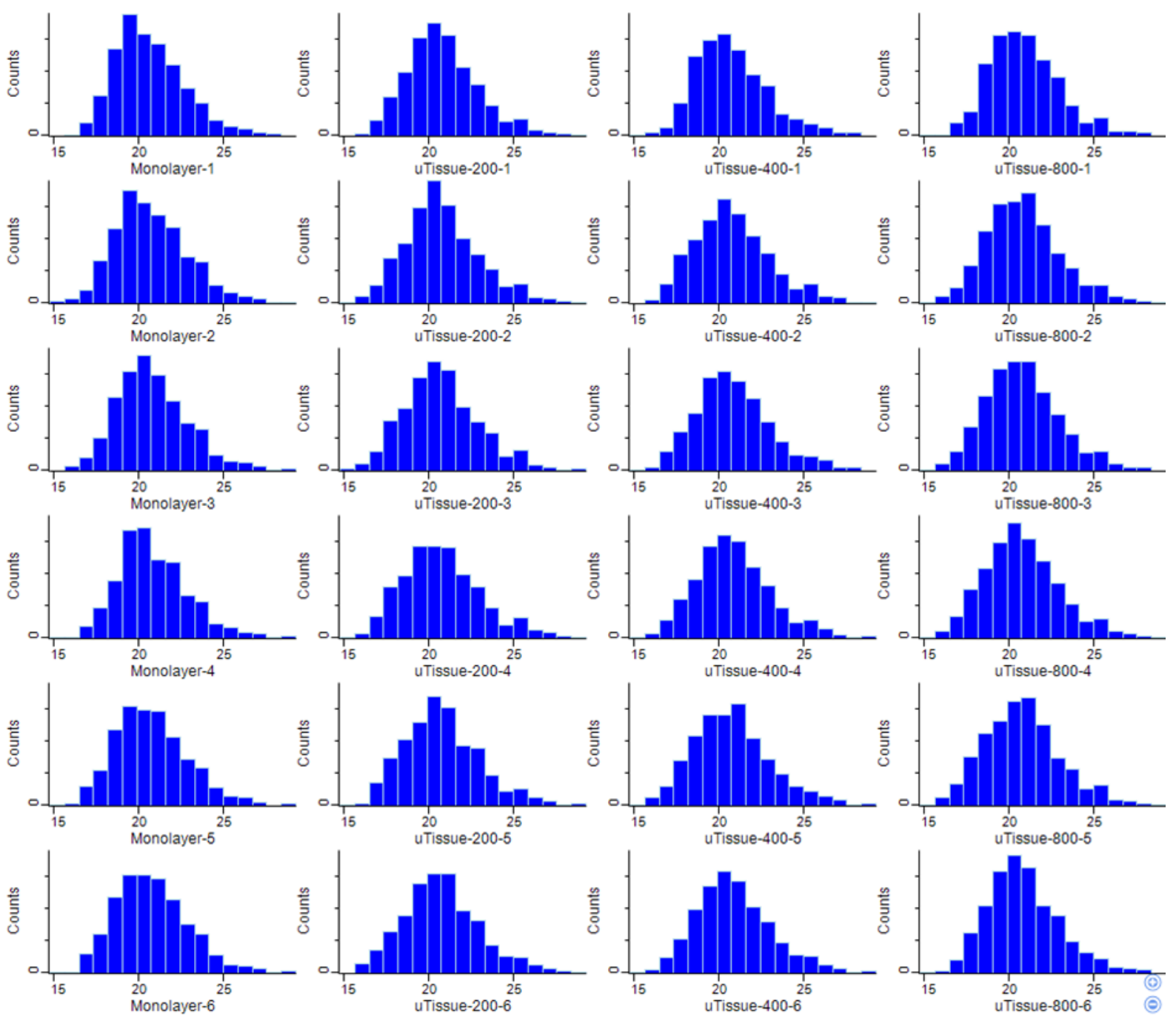

Figure 2-S2 Histogram of log2 protein intensity for each sample. Histogram plots of the proteomics data representing normal distribution in monolayers, and small (uTissue-200), medium(uTissue-400), and large (uTissue-800) MCTS, for each analytical and biological replicates. 

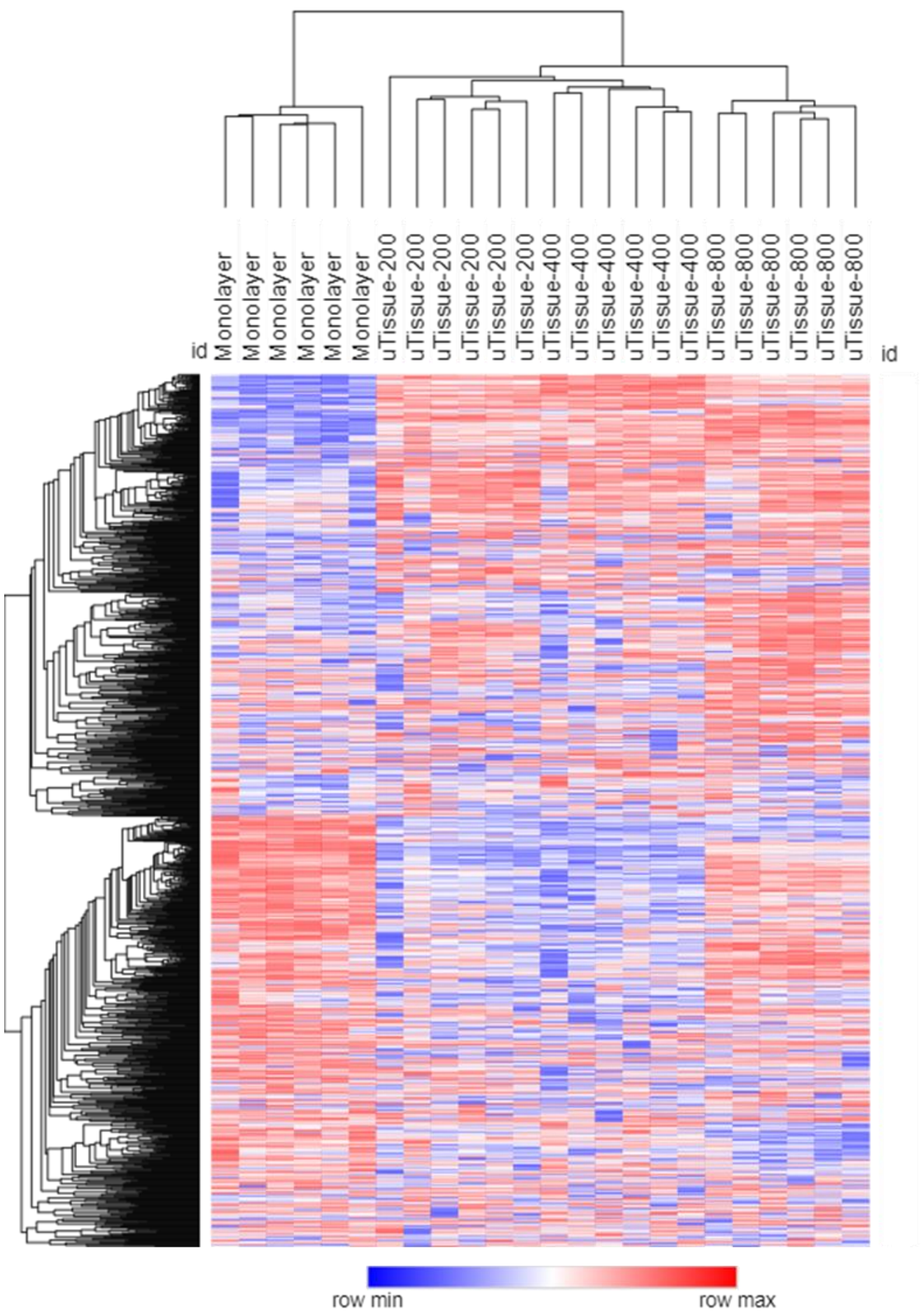

Figure 2-S3 Complete heat map of the protein expression profile of the 1244 proteins found significant when comparing monolayers and small (uTissue-200), medium(uTissue-400), and large (uTissue-800) MCTS. Each condition was prepared $2 x$ experimentally and analyzed $3 x$ per experiment. (LFQ data). 

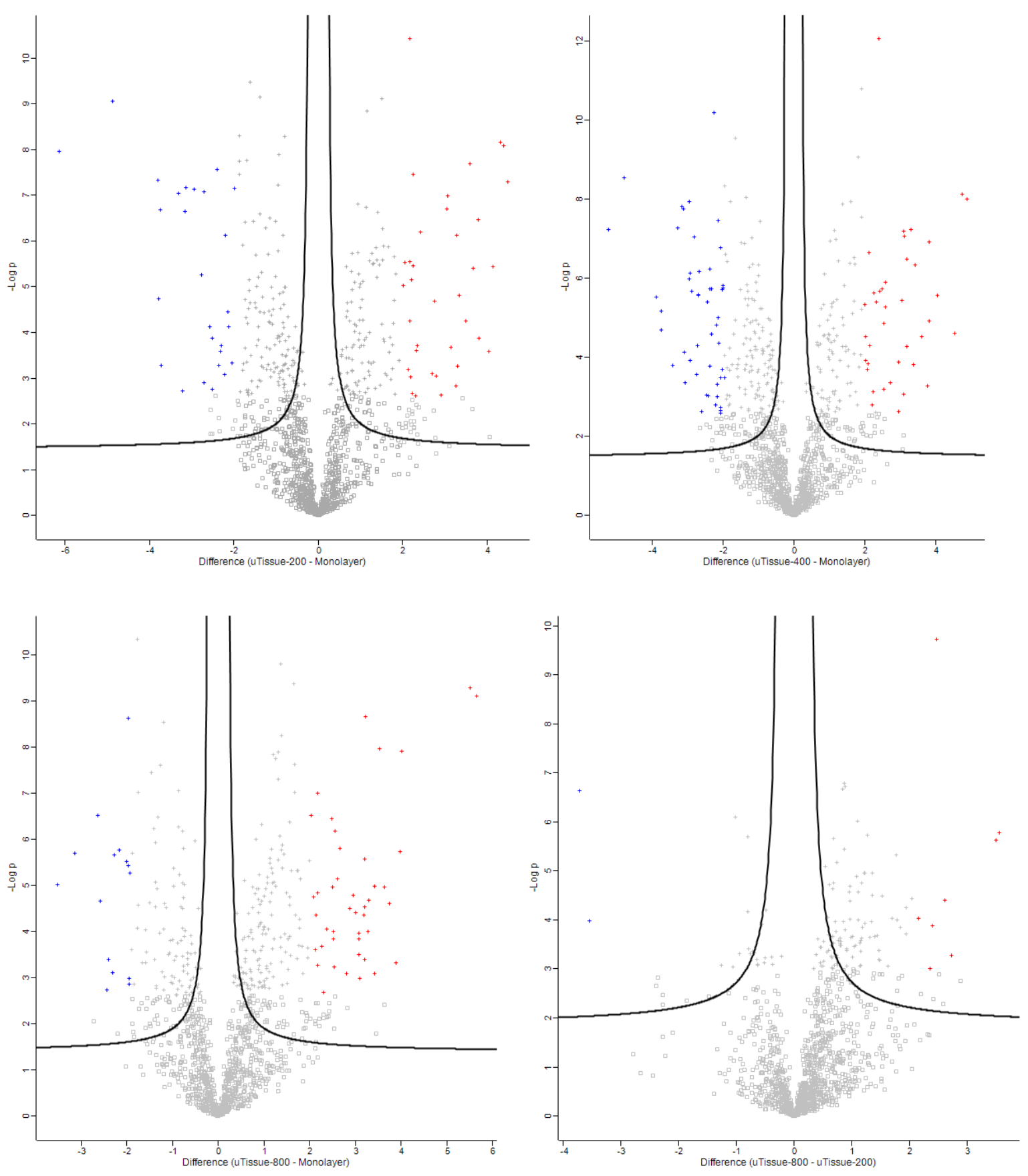

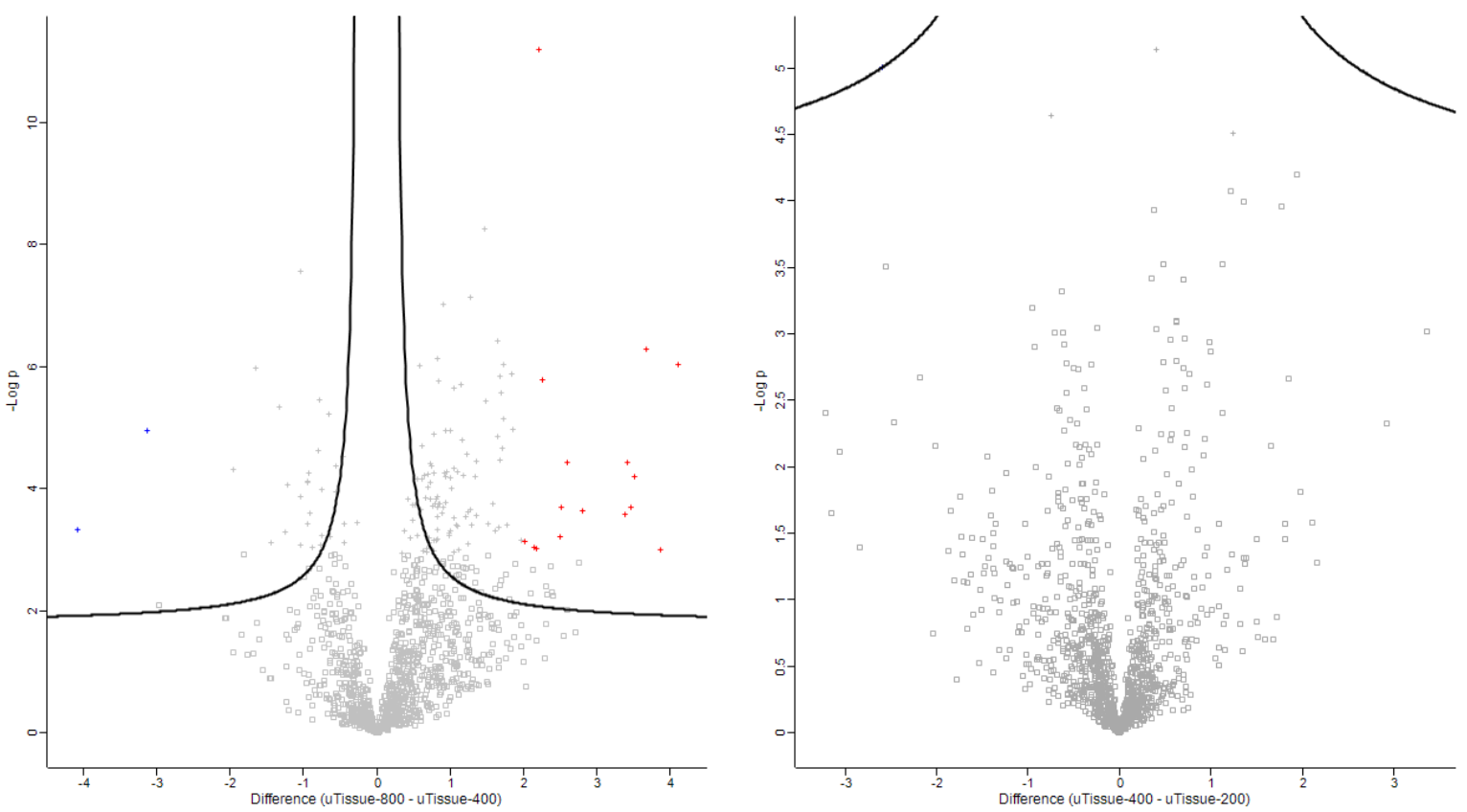

Figure 2-S4 Volcano plot reflecting the results from the statistical analysis of the 1244 proteins quantified. Statistical analysis was performed by Student t-test and statistical significance was considered for $F D R=0.01$ and $S O=0.1$. The Volcano plots corresponding to the - Log $p$ values vs. the $\log 2$ protein abundance differences between conditions (2-fold change): monolayers, small (uTissue200), medium(uTissue-400), and large (uTissue-800) MCTS. The cross corresponding of proteins which are identified with significative difference after t-test and Benjamini-Hoechberg correction. Red cross corresponding to the up-regulated proteins with fold change $\geq 2$ and blue cross down-regulated proteins with fold change $\leq-2$. 
Table 2-S1 List of the genes and their associated proteins, up-regulated $\left(F_{c}>2\right)$ or down regulated $\left(F_{c}<-2\right)$ when comparing the different tumour spheroid sizes (small 200, medium 400, large 800) with the monolayer.

\section{Up-regulated}

\begin{tabular}{|c|c|c|c|}
\hline Names & Total & Genes & Proteins \\
\hline 200400800 & 18 & $\begin{array}{l}\text { Acsl4, Tmsb4x, Spp1, Dbi, Crip1, Aldoc, } \\
\text { Pgm2;Pgm1, Tes, Idh1, Plcg2, Aldh3a1, } \\
\text { mCG_120563, Nucb1, Casp3, Vwa5a, Tgm2, } \\
\text { Hmga2, Cstb }\end{array}$ & $\begin{array}{l}\text { Q9QUJ7, P20065, P10923, Q9JI99, P31786, P63254, P05063, Q7TSV4, } \\
\text { Q9D0F9, P47226, O88844, Q8ClH5 ,P47739, Q02819, P70677, Q99KC8 } \\
\text { „P21981,P52927,Q62426 }\end{array}$ \\
\hline 200400 & 5 & Clu, Tmsb10, Ddah2, Fn1, Atp6v0d1,Marc2 & Q06890, Q6ZWY8, Q99LD8, P11276, P51863,Q922Q1 \\
\hline 400800 & 5 & Fth1, Lcn2, Stom, Arhgap1, Pafah1b3 & P09528, P11672, P54116, P54116, Q61205 \\
\hline 200 & 6 & $\begin{array}{l}\text { Hdac1;Gm10093; Hdac2, Csde1, Tor1aip1, } \\
\text { S100a4, Ado, Rbm3 }\end{array}$ & O09106, P70288, Q91W50,Q921T2, P07091, Q6PDY2, 089086, \\
\hline
\end{tabular}

\section{Down-regulated}

\begin{tabular}{|c|c|c|c|}
\hline Names & Total & Genes & Proteins \\
\hline 200400800 & 4 & Pdcd11, Ddx21, Slc3a2, Aldh18a1 & Q6NS46, Q9JIK5, P10852, Q9Z110 \\
\hline 200400 & 19 & $\begin{array}{c}\text { Timm13, Asns, Rcc1, Rrp9, Pgam5, Pycr2, Timm8a1, Nop2, } \\
\text { Rsl1d1, Pnn, Psat1, Mrpl12, Shmt2, Tpm1, Trap1, Lyar, Lonp1, } \\
\text { Pck2, Ghitm }\end{array}$ & $\begin{array}{c}\text { P62075, Q61024, Q8VE37, Q91WM3, Q8BX10, Q922Q4, Q9WVA2, } \\
\text { Q922K7, Q8BVY0, O35691, Q99K85, Q9DB15, Q9CZN7, P58771, } \\
\text { Q9CQN1, Q08288, Q8CGK3, Q8BH04, Q91VC9 }\end{array}$ \\
\hline 200800 & 0 & & \\
\hline 400800 & 2 & $\mathrm{Mcm} 4$, Acot2 & P49717, Q9QYR9 \\
\hline 200 & 3 & Samhd1, Ptrh2, Tjp1 & Q60710, Q8R2Y8, P39447 \\
\hline 800 & 4 & Ssrp1, FInb, Tcea1, Wdr43 & Q08943, Q80X90, P10711, Q6ZQL4 \\
\hline
\end{tabular}


A

Up-regulated

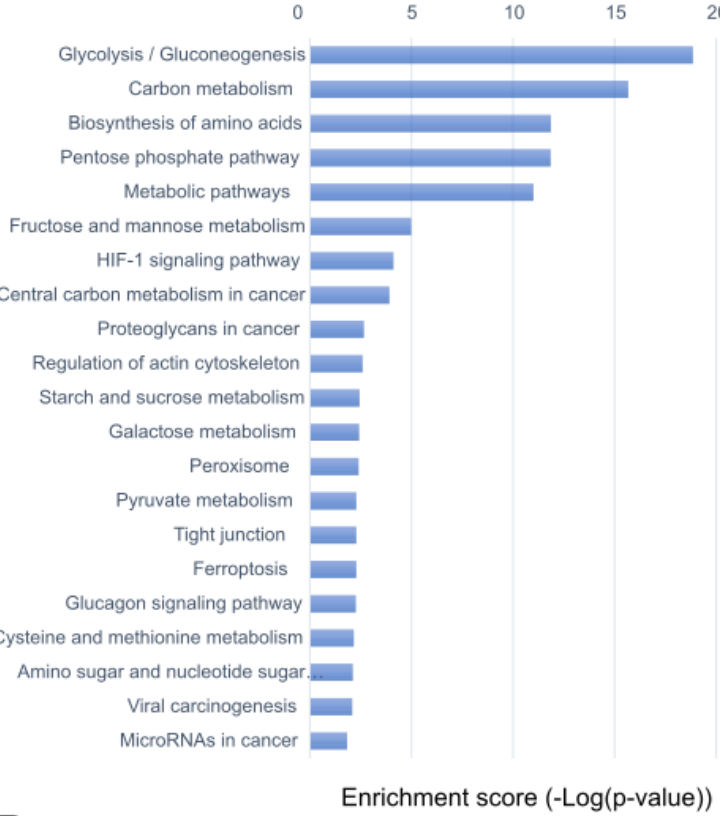

B

Molecular Functions (MF)

Up-regulated

phosphoglucomutase activity

AT DNA binding

DNA binding, bending

magnesium ion binding

binding

\section{Down-regulated}

double-stranded RNA binding RNA binding organic cyclic compound binding heterocyclic compound binding
Down-regulated

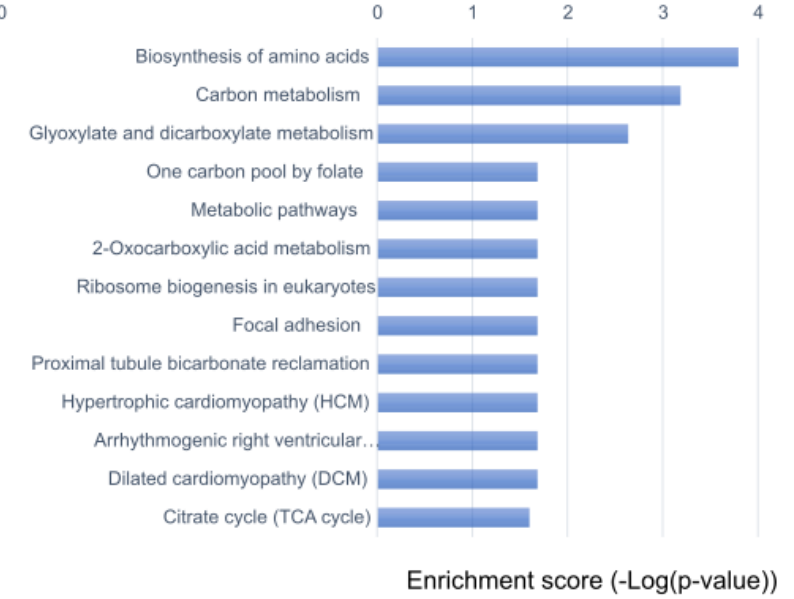

Response to nutrient

intracellular part

cytosol

cytoplasm

cytoplasmic part response to exogenous dsRNA

rRNA processing

Figure 2-S5 KEGG (A) and GO (B) pathways in the MCTS against the monolayer. A) KEGG enrichment score was determined using the log(p-value), including all pathway with a p-value $<0.01$, and indicated an increase in number of activated pathways in the MCTS. 
Table 2-S2: List of the genes associated with the proteins found up- or down regulated in all MCTS sizes, as compared to the monolayer $(|F c|>2)$.

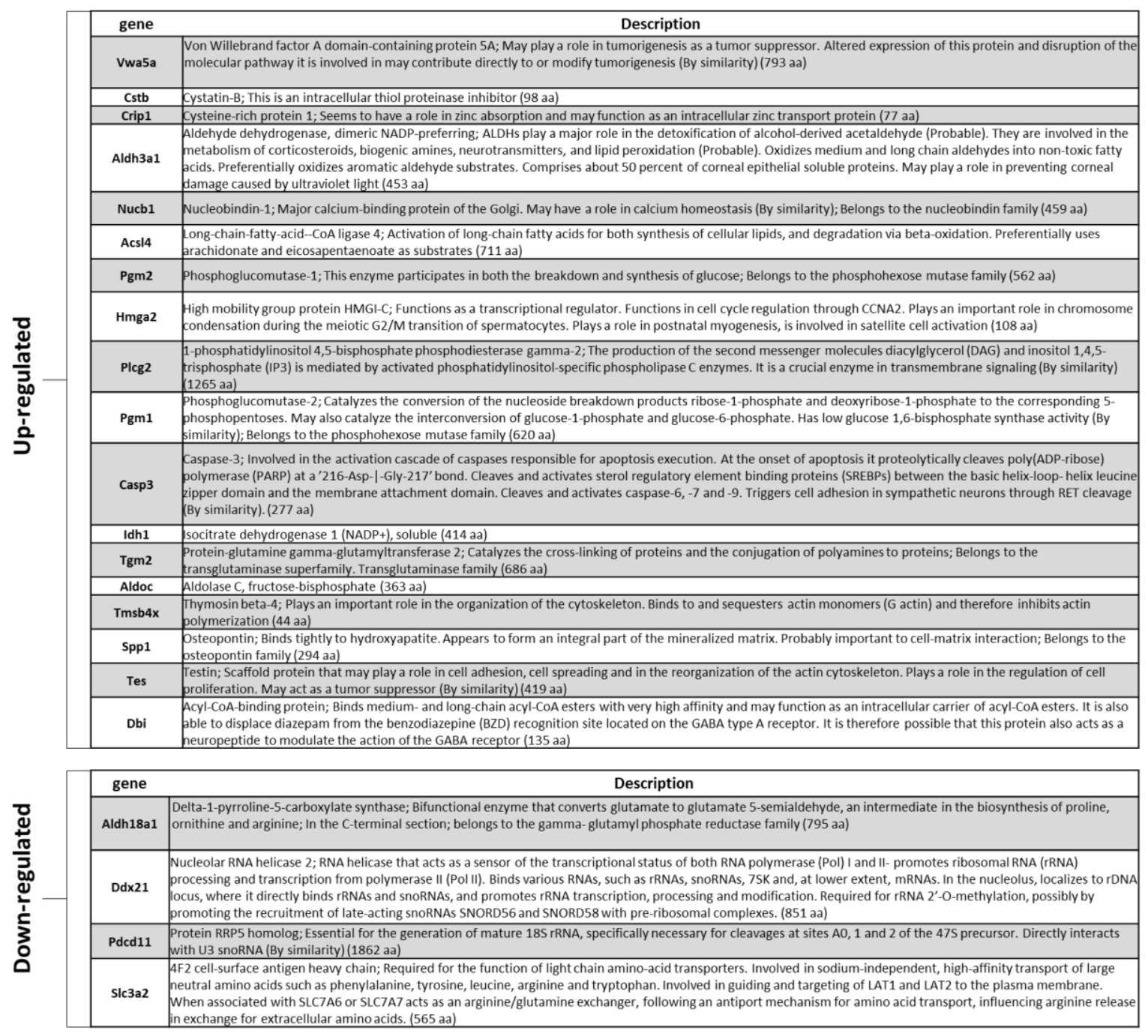


Table 2-S3 List of protein up-regulated (>) and down-regulated (<) between the different MCTS sizes (small, medium and large MCTS) after 2-fold change threshold, with the type of cancer in which they have previously been identified to play a role in tumor progression. The unidentified functions (N/A) results from the current missing studies on the specific interaction of the protein with cancer. No protein was found up-regulated in the medium MCTS when comparing the medium and small MCTS (medium MCTS > small MCTS).

\begin{tabular}{|c|c|c|}
\hline Protein & Name & Presence in tumor \\
\hline \multicolumn{1}{|l|}{ large MCTS $>$ small MCTS } & N/A \\
\hline Cbr2 & Carbonyl reducatse [NADPH] 2 & prostate, lung \\
\hline Aldh18a1 & $\begin{array}{c}\text { Aldehyde dehydrogenase 18 } \\
\text { Familiy Member A1 }\end{array}$ & N/A \\
\hline Gcat & Glycine C-acetyltransferase & oral, breast \\
\hline Tpp2 & tripeptidyl peptidase 2 & prostate, lung \\
\hline Aldh3a1 & $\begin{array}{c}\text { Aldehyde dehydrogenase 3 } \\
\text { Familiy Member A1 }\end{array}$ & melanoma \\
\hline Cdkn2c & Cyclin-dependent kinase 4 inhibitor C & Thyroid \\
\hline Timm44 & $\begin{array}{c}\text { Mitochondrial import inner membrane } \\
\text { translocase subunit TIM44 }\end{array}$ & colon \\
\hline Lonp1 & Lon protease homolog, mitochondrial & colon, cervical, pancreas \\
\hline Gstm2 & Glutathione S-transferase Mu 2 & N/A \\
\hline large MCTS $<$ small MCTS & Pleiotropic regulator 1 & various tumour, breast \\
\hline Plrg1 & Epithelial cell adhesion molecule &
\end{tabular}




\begin{tabular}{|c|c|c|}
\hline \multicolumn{3}{|c|}{ large MCTS > medium MCTS } \\
\hline Clic4 & Chloride intracellular channel protein 4 & various tumours \\
\hline Psmb7 & Proteasome subunit beta type- 7 & squamous \\
\hline Sec61a1/2 & $\begin{array}{c}\text { Protein transport protein Sec61 } \\
\text { subunit alpha isoform } 1 / 2\end{array}$ & $\mathrm{~N} / \mathrm{A}$ \\
\hline Nqo1 & NAD(P)H Dehydrogenase, Quinone 1 & various tumours, breast \\
\hline Gars & Glycyl-TRNA Synthetase & $\mathrm{N} / \mathrm{A}$ \\
\hline Fabp5 & Fatty Acid Binding protein 5 & prostate, lung \\
\hline Atl3 & Atlastin GTPase 3 & $\mathrm{~N} / \mathrm{A}$ \\
\hline Aldh18a1 & $\begin{array}{c}\text { Aldehyde dehydrogenase } 18 \\
\text { Familiy Member A1 }\end{array}$ & prostate, lung \\
\hline Ogdh & Oxoglutarate Dehydrogenase & colon, fibrosarcoma \\
\hline Nudt5 & Nudix Hydrolase 5 & $\mathrm{~N} / \mathrm{A}$ \\
\hline Myo1c & Myosin-Ic & prostate \\
\hline Tpp2 & tripeptidyl peptidase 2 & oral, breast \\
\hline Tapbp & TAP-associated glycoprotein & breast \\
\hline Aldh3a1 & $\begin{array}{c}\text { Aldehyde dehydrogenase } 3 \\
\text { Familiy Member A1 }\end{array}$ & prostate, lung \\
\hline Timm44 & $\begin{array}{c}\text { Translocase of Inner } \\
\text { Mitochnodrial Membrane } 44\end{array}$ & thyroid \\
\hline Mtch2 & Mitochondrial carrier homolog 2 & $\mathrm{~N} / \mathrm{A}$ \\
\hline \multicolumn{3}{|c|}{ large MCTS < medium MCTS } \\
\hline Epcam & Epithelial cell adhesion molecule & various tumour, breast \\
\hline Hagh & Hydroxyacylglutathione hydrolase & $\mathrm{N} / \mathrm{A}$ \\
\hline \multicolumn{3}{|c|}{ medium MCTS < small MCTS } \\
\hline Sec61a1/2 & $\begin{array}{l}\text { Protein transport protein Sec61 } \\
\text { subunit alpha isoform } 1 / 2\end{array}$ & $\mathrm{~N} / \mathrm{A}$ \\
\hline
\end{tabular}




\section{Chapter 3 - Studying the Cellular and Molecular Organization in Multicellular Tumour Spheroids using CLARITY}

\section{Abstract}

After having investigated the proteome of our multicellular tumour spheroid models, we aimed here at examining the specific location of the expression of a few selected proteins of interest in 3D. To do so, we developed a fast protocol to physically clear 3D tissues, to eliminate light scattering, based on the CLARITY (Clear Lipid-exchanged Anatomically Rigid Imaging/ immunostaining-compatible Tissue hYdrogel) and PACT (Passive CLARITY Technique) protocols established by Treweek et al.. Using microfluidics, to confine the samples, we replaced the passive incubation of the different clearing steps, proposed in PACT, with dynamic flow $(800 \mu \mathrm{L} / \mathrm{h}$ and $2820 \mu \mathrm{L} / \mathrm{h})$. We successfully cleared multicellular tumour spheroids (MCTS) of $c a .300 \mu \mathrm{m}$ in diameter, and effectively reduced the clearing time from several days to several hours, when compared to static incubation. We next applied this protocol to characterize the MCTS cellular and molecular organization by performing immunostaining on the cleared tissue. However, as a result of the use of a strong detergent (SDS) for clearing the tissue, proteins present inside the spheroids were denaturated, which precluded the antibody recognition in the immunostaining step. 


\section{INTRODUCTION}

The main challenge when studying 3D tumour models is to obtain precise cellular and molecular information deep inside the tissues. In a first conventional approach to characterize 3D tissues and (tumour) spheroids (i.e., hypoxia levels, protein expression, drug response), the tissue is fixed, embedded in paraffin followed by sectioning or cryosectioning. This method has already provided extensive knowledge on the tumour composition [1-4], but it is limited as the samples are damaged in the process, and a complete mapping of targeted proteins in the spheroids is often impossible. Alternatively, the tissue can be directly imaged, in its intact form, using confocal microscopy to access 3D information. However, antibodies normally used to stain specific targets do not penetrate efficiently the dense tissue via diffusive processes. Additionally, microscopic examination of the tissues, e.g., using confocal microscopy is hindered by the presence of the phospholipids forming the cell membranes that diffract the light.

To address these issues, tissue clearing strategies have been proposed. Notably, a novel approach named CLARITY, which stands for Clear Lipid-exchanged Anatomically Rigid Imaging/immunostaining-compatible Tissue hYdrogel has been introduced to render large tissues transparent, solving the issue of light scattering, while promoting the penetration of the staining agents [5, 6]. CLARITY consists of first creating a hydrogel polymer around and in the tissue, to which all proteins present in the tissue are crosslinked. Next, this hydrogel is placed in a solution of a strong detergent that solubilizes the (phospho)lipids into micelles. The lipids are subsequently removed either passively (diffusion only) or actively with electrophoresis to promote their migration out of the tissue. Initially developed for clearing complete organs [7], CLARITY has quickly been adapted for smaller systems, such as spheroids or 3D cellular aggregates [8, 9]. Among those, PACT (Passive CLARITY Technique) uses passive conditions (diffusion-based only) for the tissue clearing to avoid the use of an electric field, which can damage the tissues. Furthermore, the hydrogel pore size in PACT is increased to promote diffusion of the reagents [5,9]. The main limitation of this CLARITY approach remains the time required to perform the entire protocol, mainly due to the slow diffusion of all reagents, which can reach time durations longer than one week. 
In this chapter, we evaluate a protocol based on CLARITY and its PACT variant to clear multicellular tumour spheroids and next image the samples in situ, to eventually achieve a complete mapping in 3D of the expression of proteins of interest. Using microfluidic technology, we successfully decreased both the required experimental time duration (from days to hours) and the amount of chemicals needed for the different steps. As a proof-of-concept, we applied this protocol to investigate, on one hand, the cellular reorganization of cancer cells and fibroblasts in co-culture spheroids and, on the other hand, the spatial layer-by-layer structure of the spheroids, using specific proliferation, quiescence and hypoxic markers. Finally, we examined how the size and cell composition of the spheroids would affect the clearing processes and the expression levels of the proteins considered in this study.

\section{MATERIAL AND METHODS}

\subsection{Cell Culture}

4T1 mouse breast cancer cells and 3T3 mouse fibroblasts were purchased from the American Type Culture Collection (ATCC, Rockville, MD). 4T1 and 3T3 were respectively cultured in Roswell Park Memorial Institute (RPMI) 1640 medium and Dulbecco's Modified Eagle's Medium (DMEM), both supplemented with $2 \mathrm{mM} \mathrm{L-glutamine,} 10 \%$ fetal bovine serum (FBS) and antibiotics (50 U.mL $\mathrm{mL}^{-1}$ Penicillin and $50 \mathrm{ng} \cdot \mathrm{mL}^{-1}$ streptomycin). Both cell types were expanded in $75-\mathrm{cm}^{2}$ culture flasks (CELLSTAR® Filter Cap $75 \mathrm{~cm}^{2}$, GREINER BIO-ONE, Kremsmünster, Austria) in an incubator under standard humidified conditions $\left(37^{\circ} \mathrm{C}, 5 \% \mathrm{CO}_{2}\right)$. Medium was refreshed every 2 days, as described thereafter. Briefly, to retrieve the cells from the flask, medium was removed, and cells washed with sterile PBS 1X buffer (Lonza, Basel, Switzerland) to remove dead (floating) cells. $2 \mathrm{~mL}$ of trypsin-EDTA $(0.25 \%)$ (Thermofisher, Netherlands) was added for $5 \mathrm{~min}$ to detach the cells from the surface. The cells were next resuspended in $8 \mathrm{~mL}$ of fresh DMEM medium to block the action of the trypsin, and collected in $10 \mathrm{~mL}$ tubes. After centrifugation at $300 \mathrm{~g}$ for $3 \mathrm{~min}$, medium was removed and $2 \mathrm{~mL}$ of fresh DMEM medium was added. Cells were counted and $c a .2$ million cells were seeded in a new culture flask. The remaining cells were kept for further experiments. 


\subsection{SPHEROID PREPARATION}

Tumour microtissues were prepared as previously reported by us [10], and presented in the previous chapters. Arrays of microwells (either $400 \mu \mathrm{m}$ diameter x $200 \mu \mathrm{m}$ depth, or 800 $\mu \mathrm{m}$ diameter $\mathrm{x} 500 \mu \mathrm{m}$ depth) were prepared in a $35-\mathrm{mm}$ diameter Petri dish using softembossing and a PDMS mold. After embossing, the petri dish was sterilized with a $70 \%$ ethanol solution and incubated overnight with a $1 \% \mathrm{w} / \mathrm{w}$ Pluronic F-127 solution. Pluronic was then removed and replaced with culture medium. About 2.5 million cells were injected per Petri dish and forced into the microwells by centrifugation (1800 g, $5 \mathrm{~min}$ ). The excess of cells was removed with PBS, and the devices were incubated $\left(37^{\circ} \mathrm{C}, 5 \% \mathrm{CO}_{2}\right)$ in medium for $42 \mathrm{~h}$, until cells fully aggregated as spheroids. The resulting spheroids were pipetted out of the Petri dishes and placed in an Eppendorf microtube, where they were washed with PBS and, when needed, prefixed with a $4 \%$ formaldehyde solution for $1 \mathrm{~h}$ at room temperature.

\subsection{TISSUE CLEARING}

Otherwise stated, reagents were purchased from SIGMA-ALDRICH (Zwijndrecht, The Netherlands). CLARITY protocols were inspired by the method described by R. Tomer et al. [11].

\subsubsection{Hydrogel embedding solution}

The hydrogel monomer solution was prepared on ice by mixing acrylamide, formaldehyde, bis-acrylamide, and the azo initiator 2,2'-Azobis<2-(2-imidazolin-2yl)propane> dihydrochloride (VA-044, Wako Chemicals GmbH, Neuss, Germany). In the original protocol, the embedding solution was prepared with $4 \%$ acrylamide, $4 \%$ formaldehyde, and $0.05 \%$ bis-acrylamide, which is abbreviated as A4F4B0.05. In this work, the concentrations of the different solutions were varied to evaluate the impact of the different chemicals on both the spheroid clearing and immunostaining. Using A4F4B0.05 as a reference, the influence of the bis-acrylamide concentration on prefixed tissues was investigated. Since here pre-fixed tissues were considered, formaldehyde was omitted from the hydrogel formulation. Therefore, the following mixtures were considered: A4F0B0.025, A4F0B0.0125 and A4F0B0. To evaluate the influence of the formaldehyde incubation time on the polymer 
formation, the same experiment was performed on unfixed tissues using the same hydrogel formulations, yet supplemented with 4\% formaldehyde: A4F4B0.025, A4F4B0.0125 and A4F4B0. In all experiments, the azo initiator was added to the mixture, at a final concentration of $0.25 \% \mathrm{w} / \mathrm{v}$, just before adding the solution to the spheroids, to avoid pre-polymerization of the hydrogel.

\subsubsection{Clearing solution}

The clearing buffer was prepared by mixing together boric acid with the detergent sodium dodecyl sulphate (SDS). Boric acid was first dissolved in water to reach a $1 \mathrm{M}$ concentration and the $\mathrm{pH}$ adjusted with sodium hydroxide $(\mathrm{NaOH}, 1 \mathrm{M})$ to 8.5 , as evaluated with a $\mathrm{pH}$ meter. SDS was next dissolved in the boric acid solution. The concentration of SDS was varied from $1 \%$ to $8 \% \mathrm{w} / \mathrm{v}$ to investigate its impact on the lipid removal efficiency.

\subsubsection{Static clearing}

After their introduction in the embedding solution (on ice), the spheroids were placed in the fridge for $24 \mathrm{~h}$ or $48 \mathrm{~h}$ to allow the monomers to diffuse inside the tissue. Next, since oxygen inhibits the polymerization reaction, samples were degassed in a desiccator, and purged with nitrogen gas. Polymerization was initiated by placing the sealed oxygen-free samples in a water bath $\left(37^{\circ} \mathrm{C}\right)$ with gentle agitation for $3 \mathrm{~h}$.

Following this, the spheroids were pipetted from the hydrogel embedding solution, washed with $0.1 \%$ PBS Triton-X100 (PBST) and incubated in the clearing solution for $24 \mathrm{~h}$ (refreshed every few hours). The impact of the incubation conditions in the clearing solution was investigated and the following temperatures were considered: $20^{\circ} \mathrm{C}$ (room temperature), $37^{\circ} \mathrm{C}$, and $55^{\circ} \mathrm{C}$. Once the clearing was completed, the samples were washed with $0.1 \%$ PBST.

\subsubsection{Transparency measurement}

To evaluate the tissue spheroid transparency in bright light, a pattern of grid lines was printed on a transparent sheet, cut, and deposited at the bottom of a well plate. Spheroids were then placed on top of the grid and their transparency was evaluated. 


\subsubsection{Dynamic clearing}

To accelerate the different steps of the previously described protocol and to promote the penetration of the chemicals into the samples, a perfusion-assisted approach was considered. After static prefixation in a 4\% formaldehyde solution, 3-5 spheroids were placed in a microfluidic device designed with a chamber and a trapping structure to maintain the spheroids in the chamber upon perfusion, and subsequently expose them to various flows of chemicals.

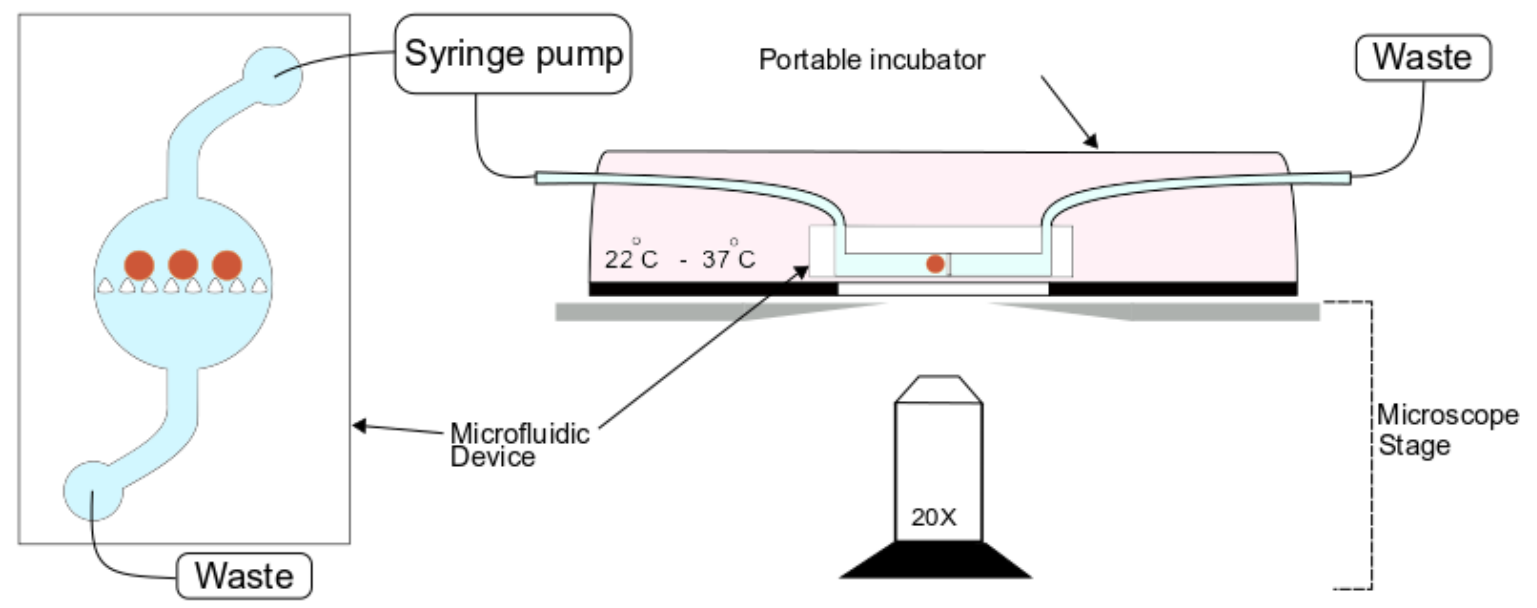

Figure 3-1 Microfluidic setup. On the left, top-view schematic representation of the microfluidic device loaded with 3 spheroids, connected to the syringe pump via its inlet, and a wasts disposal system via its outlet. On the right, side-view representation of microfluidic device placed in a portable incubator to control the temperatire and gas tension, installed on a microscope stage.

A PDMS (polydimethylsiloxane) pre-polymer was mixed with a curing agent, in a 10:1 weight ratio solution. This solution was poured on a silica-SU8 wafer mold, degassed, and cured at $60^{\circ} \mathrm{C}$ for $3 \mathrm{~h}$. Detailed protocols for preparing molds and devices are described in chapter 5. The PDMS device was next peeled off, cut to size, and inlets and outlets were punched using a 1-mm in diameter puncher (Integra Miltex, York, USA). Finally, the PDMS structure was plasma bonded to a glass coverslip (\#1.5, VWR, Radnor, USA). Up to four microfluidic devices were placed in a portable incubator (stage top incubator, Ibidi, Gräfelfing, Germany), connected with Microbore Tubing (Cole-Parmer, Vernon Hills, USA) in series together and to a 1-mL glass Hamilton syringe placed on a syringe-pump (Harvard Apparatus, Pump 11 Elite) via the inlets and to a waste disposal system via the outlets (Figure 3-1).

The embedding solution was kept cold in the syringe using ice pack and perfused for $20 \mathrm{~min}$ at $60 \mu \mathrm{L} / \mathrm{h}$ in the devices. The portable incubator was turned on $\left(37^{\circ} \mathrm{C}\right)$ and the devices 
incubated statically (no flow) for $2 \mathrm{~h}$. The spheroids were next washed with a $0.1 \%$ PBST solution for $20 \mathrm{~min}$ at $60 \mu \mathrm{L} / \mathrm{h}$. The clearing solution was perfused at $37^{\circ} \mathrm{C}$, at either $800 \mu \mathrm{L} / \mathrm{h}$ of $2820 \mu \mathrm{l} / \mathrm{h}$, to investigate the influence of the flow rate on the clearing. The tissues and devices were finally washed with a $0.1 \%$ PBST solution at $60 \mu \mathrm{l} / \mathrm{h}$ for $20 \mathrm{~min}$. The choice for these different flow-rates is explained in the Result section.

\subsubsection{Tissue staining}

To investigate the cellular organization, Pierce rabbit E-cadherin/CDH1 polyclonal primary antibody (\#PA5-32178, Thermo Fischer Scientific, Waltham, MA, USA) was used to stain the membranes all cells, and $\alpha$-smooth muscle actin antibody ( $\alpha$ SMA, \#PA5-16697, Thermo Fischer Scientific) to stain the fibroblasts only. To evaluate the spatial layer-by-layer structure of the spheroids, different markers specific for these different layers were considered: Ki-67 (antibody \#ab15580, abcam, UK) to stain the proliferating layer; p27 Kip-1 (polyclonal antibody \#PA5-27188, Thermo Fischer Scientific) for the quiescent layer, and Carbonic Anhydrase IX (polyclonal antibody \#PA1-16592, Thermo Fischer Scientific) for the hypoxic/necrotic core. As secondary antibodies, Goat anti-Rabbit IgG $(\mathrm{H}+\mathrm{L})$ Highly CrossAdsorbed Secondary Antibody, Alexa Fluor 488 (\#A-11034, Thermo Fischer Scientific) or Alexa Fluor 568 (\# A-11036, Thermo Fischer Scientific) were employed after incubation of the primary antibodies and subsequent washing. Both the primary and secondary antibodies were prepared at a dilution of 1:100. As a control, small molecules were also tested: Propidium Iodide (PI, \#P3566, Thermo Fischer Scientific) and Hoechst 33342 (NucBlue, \#R37605, Thermo Fischer Scientific) as nuclear counterstain, and phalloidin (Actin Green, \#R37110, Thermo Fischer Scientific) as a standard staining reference.

Under static conditions (no flow), the small molecules - e.g. phalloidin (788 Da), Hoechst 33342 (452 Da) and Propidium Iodide (668 Da) - were added to the spheroids and incubated overnight in a 3\% w/w Bovine Serum Albumin (BSA) solution in an Eppendorf microtube. For immunostaining, the primary and secondary antibodies were successively incubated each for at least $24 \mathrm{~h}$ in the same $3 \% \mathrm{w} / \mathrm{w}$ BSA solution, each followed by a washing step with $0.1 \%$ w/w PBST for $24 \mathrm{~h}$.

Under dynamic conditions the small molecules were perfused separately for 20 min up to $4 \mathrm{~h}$. For immunostaining and for both the primary and secondary antibodies, combinations of different perfusion times and static incubation times were considered to optimize the 
penetration and staining of the tissues. Each staining step was performed at either $800 \mu \mathrm{L} / \mathrm{h}$ or $2820 \mu \mathrm{L} / \mathrm{h}$, separated by a washing step at either $60 \mu \mathrm{L} / \mathrm{h}$ or $800 \mu \mathrm{L} / \mathrm{h}$ with $0.1 \%$ PBST for 15 $\min$.

After staining, the spheroids were immersed in FocusClear ${ }^{\mathrm{TM}}$ (Cedarlane Corporation, Burlington, USA), a Refractive Index matching solution, to enhance the imaging resolution. Under static conditions, the spheroids were incubated for $1 \mathrm{~h}$ in this solution prior to imaging, while in the dynamic approach, the FocusClear ${ }^{\mathrm{TM}}$ solution was pipetted in the devices and incubated statically for $15 \mathrm{~min}$, prior to imaging.

\subsubsection{Imaging}

Samples obtained from the passive clearing method (no flow) were imaged using a Zeiss confocal microscope and a 20x objective and $488 \mathrm{~nm}$ and $561 \mathrm{~nm}$ lasers, for the green and red stains, respectively. Samples obtained from the dynamic clearing were imaged using an A1 Nikon confocal microscope and 10x and 20x objectives, using three different lasers at $405 \mathrm{~nm}, 488 \mathrm{~nm}$ and $561 \mathrm{~nm}$. For each image $<20-\mu \mathrm{m}$ thick slices were acquired at mid-height of the spheroid, to determine the penetration depth of the staining agents from the edge of the spheroids.

\section{RESUlts \& DisCUSSION}

\subsection{SPHEROID FORMATION}

As presented in chapter 2, after $48 \mathrm{~h}$ incubation in the petri dish equipped with a microwell array at $37^{\circ} \mathrm{C}$, the cells aggregated into homogeneously-sized spheroids. Here, we evaluated two different sizes of microwells (400 $\mu \mathrm{m}$ and diameter $200 \mu \mathrm{m}$ in depth, and 800 $\mu \mathrm{m}$ diameter and $500 \mu \mathrm{m}$ in depth), with the motivation to study the impact of the spheroid size on its molecular and cellular organization: specifically, by increasing the spheroid size, we aimed at better mimicking certain functionalities found in vivo, such as the presence of gradients of nutrients and oxygen, leading to the presence of a hypoxic/necrotic core. According to the literature, it was estimated that a spheroid of at least $500 \mu \mathrm{m}$ in diameter would present necrosis in its core [12]. By using larger microwells we successfully produced tissues of $520 \pm 20 \mu \mathrm{m}$ in diameter with the co-culture of 3T3 murine fibroblasts and 4T1 mouse 
breast cancer cells, introduced in an initial 5:1 ratio. For the cancer cells only tissues however, the spheroids only reached a diameter of $320 \pm 20 \mu \mathrm{m}$, with either microwell array. Any cell proliferation would not result in a spheroid growth but rather in the formation of smaller aggregates that were expelled out of the microwells. Gunther et al. investigated the growth and shedding of 4T1 tumour spheroids, and showed a slow increased of the spheroid diameter, reaching ca. $500 \mu \mathrm{m}$ after 12 days of culture, often breaking down into sub-spheroids of smaller sizes [13].

\subsection{TISSUE CLEARING}

\subsubsection{Static incubation}

\subsubsection{Protein polymerization}

In order to protect the integrity of the spheroid structure and their protein content during the clearing process, the spheroids were first embedded with a monomer solution, which was later polymerized as a hydrogel to which cross-linked with the proteins. Cross-linking was achieved thanks to the presence of formaldehyde that acted as a linking agent between the proteins and the monomers, as depicted on Figure 3-2. Here, by varying the amount of acrylamide, formaldehyde, and bis-acrylamide in the embedding solution, we aimed at optimizing the polymer hydrogel formulation to retain the overall spheroid structure, while promoting the diffusion of the detergents and the antibodies, which are typically $100 \mathrm{kDa}$ in size [9]. As a reference, we first followed the protocol reported by Tomer et al., which corresponds to a A4F4B0.05 hydrogel formulation. In our hands, such a composition led to a complete polymerization of the monomers inside the tissue, but also of the surrounding solution. The spheroids being fragile, extracting them from the external hydrogel without damaging them was nearly impossible.

By twice decreasing the concentration of bis-acrylamide in the embedding solution (A4F4B0.025), polymerization still occurred inside the tumour spheroid, but the surrounding solution remained liquid, and became highly viscous. By further decreasing the bis-acrylamide concentration by a factor of 2 (A4F4B0.0125), the supernatant was sufficiently liquid to allow extracting the spheroids. It was also suggested in the literature that the polymer could be generated without any bis-acrylamide [12], and so, as a control, the embedding solution was also prepared with acrylamide and formaldehyde only (A4F4B0). 
In the initial protocol, formaldehyde was added in the polymerization solution, to simultaneously fixate and embed the spheroids in the hydrogel matrix. Fixation being the first step of any staining protocol, we decided to also test a two-step protocol consisting in a prefixation of the spheroids in a $4 \%$ formaldehyde solution, followed by the spheroid embedding in the hydrogel with cross-linking of all proteins in absence of formaldehyde (A4F0B0.125 and A4F0B0). Prefixation of the tissue did not seem to alter the integrity of the generated hydrogel, and in presence of bis-acrylamide, prefixed tissues (A4F0B0.125) were easier to handle than their unfixed counterparts (A4F4B0.125).

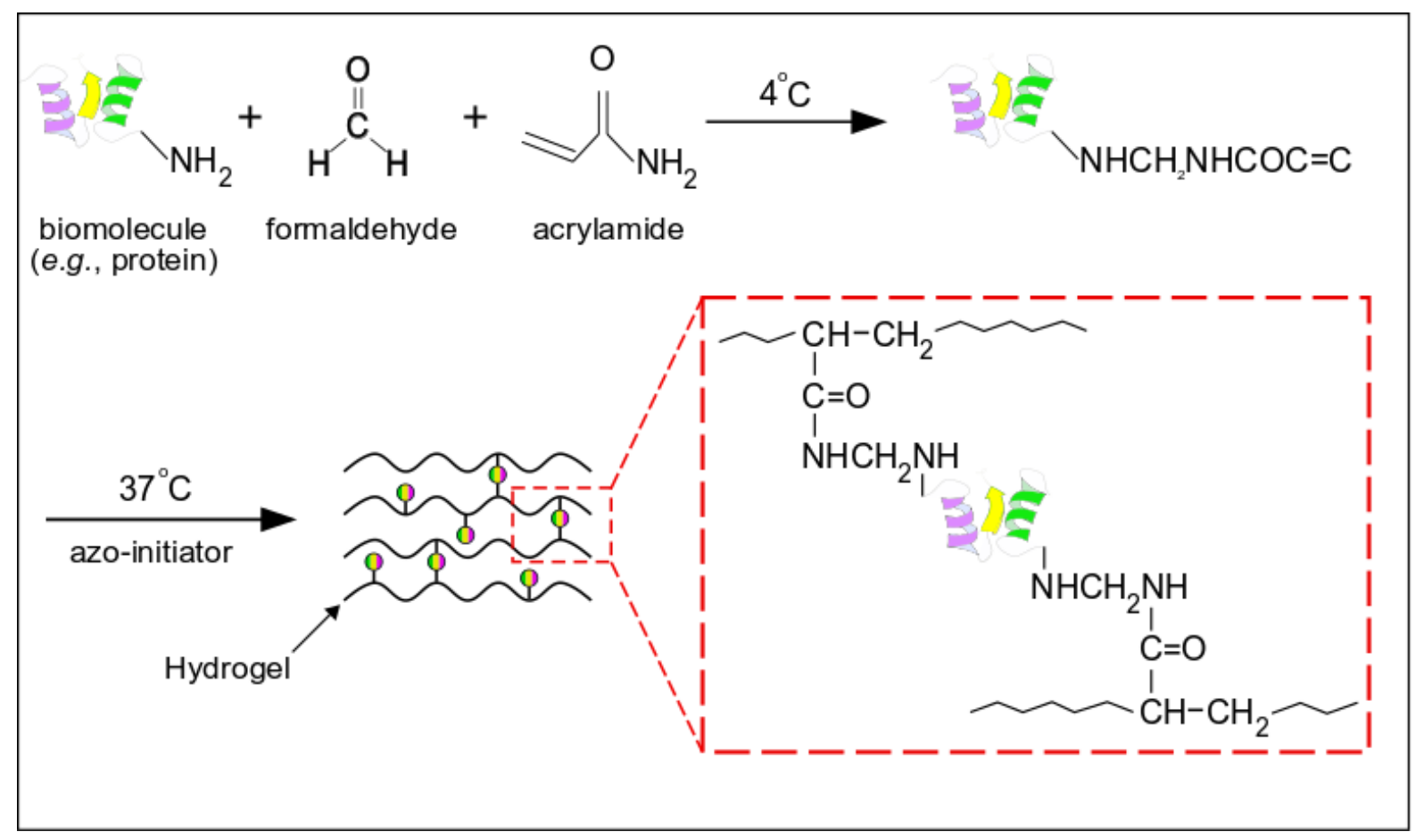

Figure 3-2 Chemical processes involved in the embedding and polymerization of the proteins with acrylamide monomers. Formaldehyde acts as a linking agent between the monomer and free amine groups found lysine residues on the protein. Upon heating, the azo-initiator present in the solution decomposes into carbon radicals, starting a radical chain reaction polymerization between the proteinmonomer complexes.

From the different embedding solution compositions available, four were retained (A4F4B0, A4F4B0.125, A4F0B0 and A4F0B0.125) based on their suitability to retrieve the spheroids after hydrogel embedding. To ascertain which conditions were better suited, all four compositions were prepared and compared after clearing. 


\subsubsection{Tissue clearing}

Once the proteins were cross-linked to the hydrogel polymer, the unbound phospholipids - which are responsible for the scattering of the lights - were removed from the tissues by immersing the spheroids in an SDS solution, which is called the clearing step. In the initial CLARITY protocol, a $4 \% \mathrm{w} / \mathrm{v}$ SDS solution was used at $50^{\circ} \mathrm{C}$ for lipid removal. In the alternative PACT approach, clearing was performed with an $8 \%$ SDS solution at $37^{\circ} \mathrm{C}$. Using either concentration (4\% and $8 \% \mathrm{w} / \mathrm{v}$ SDS) with a A4F4B0 composition for $24 \mathrm{~h}$, comparable levels of transparency was achieved (Figure 3-S1.a).

The clearing efficiency was next tested for the different hydrogel compositions. Results indicated that prefixed tissues cleared faster than the concomitantly fixed and hydrogelembedded ones (Figure 3-3.d). Also, the presence of bis-acrylamide in the hydrogel composition (A4F0B0.125 and A4F4B0.125) indicated a slower clearing and less swelling of the spheroid (Figure 3-S1.b).

Noteworthy, the spheroid size greatly affected the clearing time, to reach maximum transparency. The small mono-culture spheroids (300-400 $\mu \mathrm{m}$ in diameter) cleared much faster, ca. $32 \mathrm{~h}$, than the large co-culture ones (800-900 $\mu \mathrm{m}$ in diameter), which needed up to $156 \mathrm{~h}$ (Figure 3-S1.b.c), as expected due to the increased penetration required for the SDS to extract the lipids. Moreover, after $24 \mathrm{~h}$ clearing, the small (mono-culture) spheroids became almost completely transparent (70-80\%), while the large ones (co-culture) only achieved 50\% transparency, as indicated by the clear outer layer of the spheroid, and their dark center (Figure 3-3.g), after $33 \mathrm{~h}$ of incubation. Increasing the incubation time further did not improve the transparency level. We hypothesized that the time required to achieve maximum transparency in the large spheroids was not only due to the spheroid size, but also to the cellular composition. The presence of stroma, which includes a high amount of extracellular matrix (rich in protein content), most likely resulted in the formation of a very dense polymer network, which 
altogether significantly slowed down the penetration of SDS inside the spheroid tissues (Figure 3-S1.b.c).

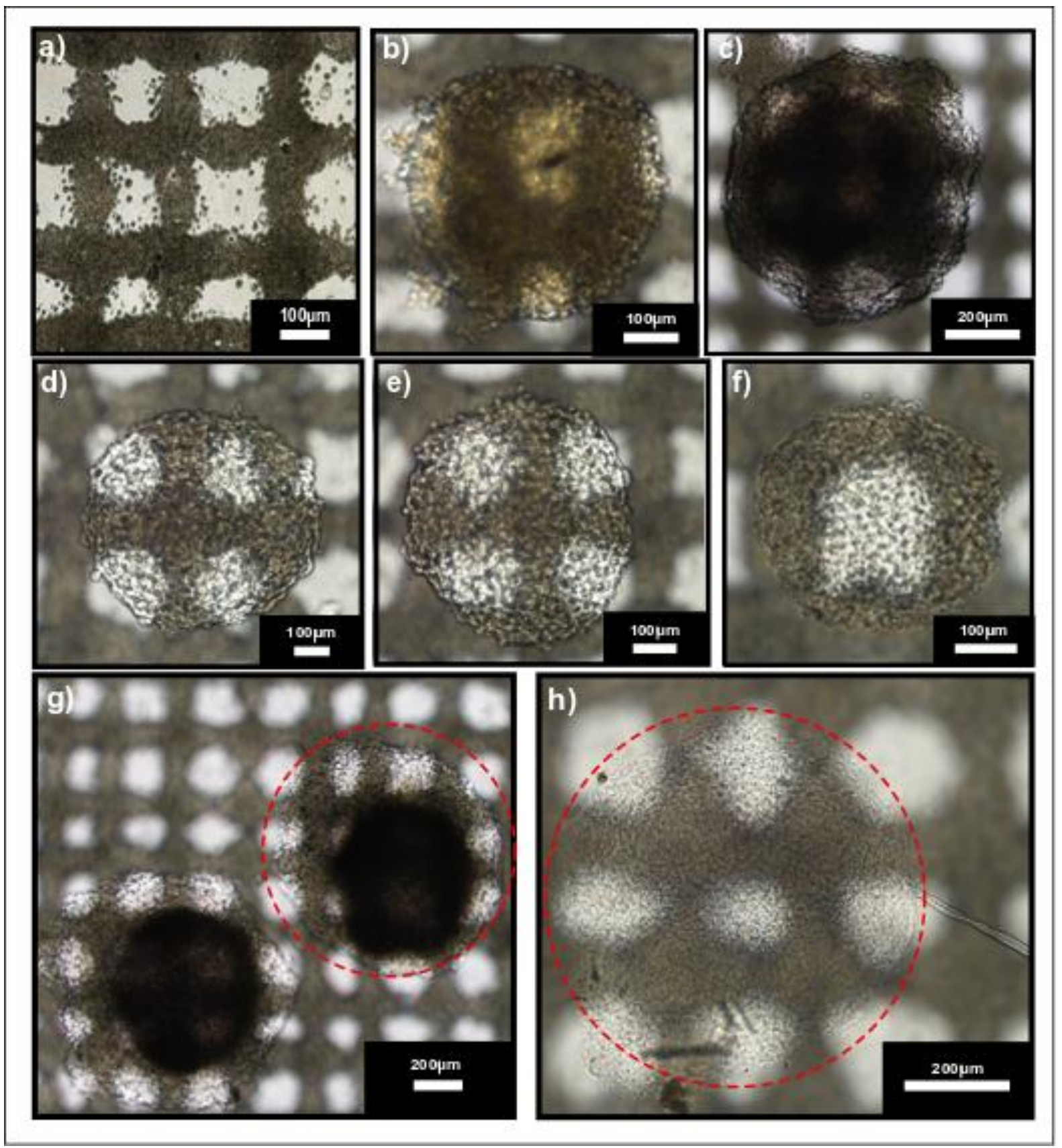

Figure 3-3 a) Grid printed on a transparent polystyrene sheet to quantify the transparency of the tissues. b) Uncleared cancer mono-culture spheroid. c) Uncleared co-culture spheroid. d) Prefixed cancer mono-culture spheroid embedded in acrylamide only (A4FOBO) after 24 h clearing. e) Prefixed cancer mono-culture spheroid, embedded in both acrylamide and bis-acrylamide (A4F0B0.125) after $24 \mathrm{~h}$ clearing. f) Prefixed cancer mono-culture spheroid cleared and immersed in Focus Clear. g) Prefixed co-culture spheroid (A4FOBO) after 33 h clearing. h) Prefixed co-culture spheroid (A4FOBO) after $33 \mathrm{~h}$ clearing and immersed in Focus Clear. 
The incomplete transparency $(<100 \%)$ of the tissues is due to the mismatching refractive index between the tissues and the supernatant, which became non negligible for the large co-culture spheroids. By adding Focus Clear, the refractive index matching solution, the mismatching was corrected to yield higher transparency level, reaching up to $100 \%$ transparency for both small and large spheroids (Figure 3-3.h).

Interestingly, throughout the clearing steps, the increase in transparency was accompanied by an increase in size of the spheroids, which were swelling up to almost $70 \%$ of their initial size (Figure 3-S1.b.c). This increase in size could be explained by the removal of lipids, leading to an osmotic pressure difference between the inside and the outside the tissues. When the clearing solution was replaced by the Focus Clear solution, the tissues shrunk back and reverted to or close to their initial size (Figure 3-S1.b.c).

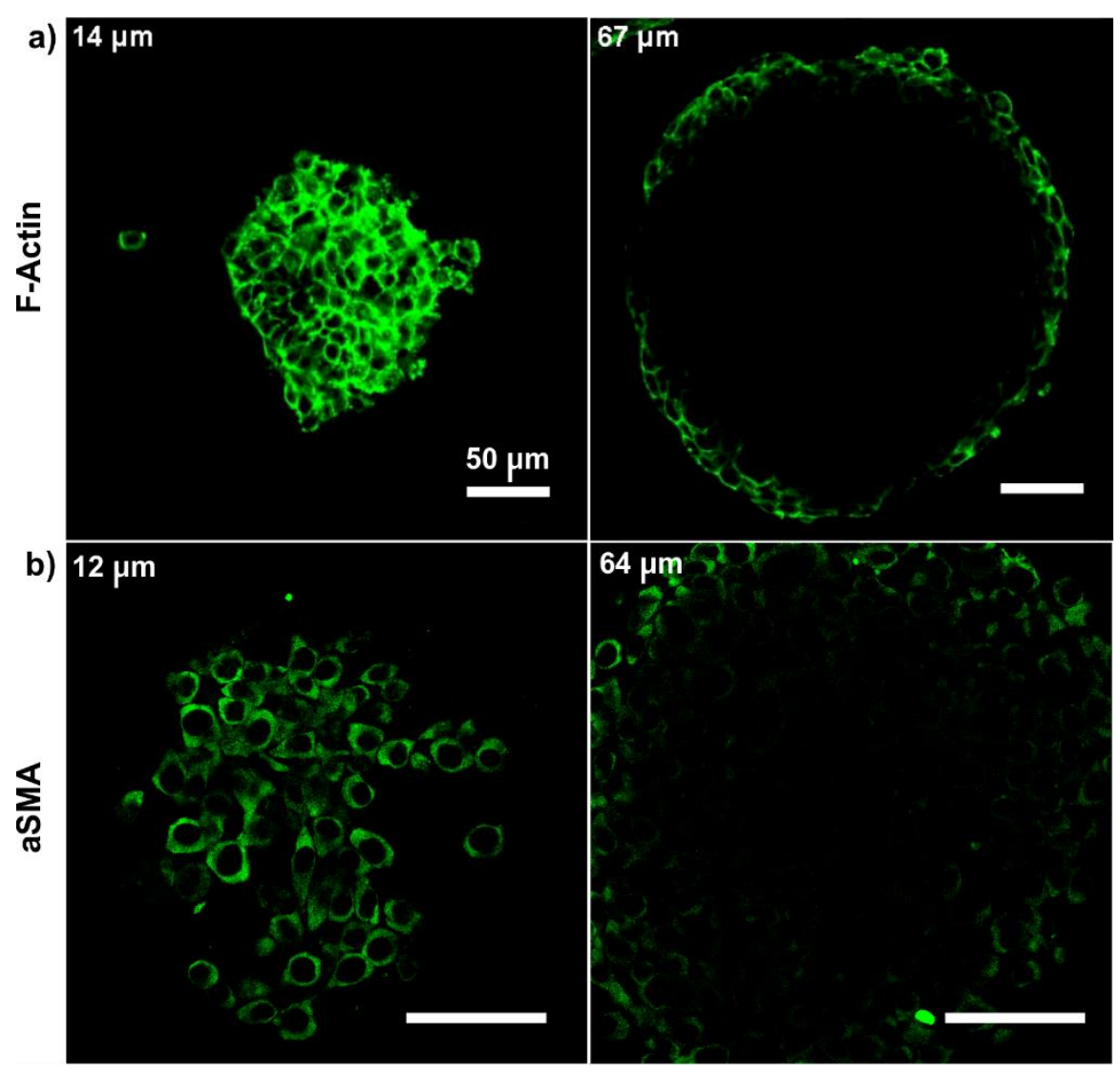

Figure 3-4 Uncleared monoculture spheroids, stained with (a) F-Actin or (b) aSMA, and imaged using confocal microscopy, at the bottom and middle section of the spheroid.

Based on the results of these experiments, it was decided to clear prefixed spheroids using the A4F0B0 monomer solution followed by the $8 \%$ SDS solution (at $37^{\circ} \mathrm{C}$ ), as these 
parameters yielded complete transparency of the tissues, and the polymerized spheroids were "easy" to handle.

\subsubsection{Fluorescent staining}

Phalloidin is a highly specific marker to F-actin, a protein found in all eukaryotic cells and involved in many cellular processes such as muscle contraction, cell motility, and cell division, which also serves as a reference marker of the cell morphology [14]. Therefore, a first assessment of the effect of tissue clearing on the spheroid staining was performed with phalloidin, in combination with Propidium Iodide (PI), a nuclear counterstain. Imaging with confocal microscopy of the uncleared tissues indicated a highly specific binding of phalloidin, clearly visible at the edge of the spheroids (Figure 3-4a). The imaging depth was however limited by the scattering of the light, and the core of the spheroid appeared as dark.

After tissue clearing, the fluorescent signal could be observed throughout the entire tissue (Figure 3-5). The sharpness of actin staining however decreased significantly, suggesting unspecific staining (Figure 3-S2). Nuclear staining remained visible in the whole tissue, but the sharpness and brightness in the core slightly decreased with the depth, suggesting mild scattering of the light from the tissue by the polymer itself.

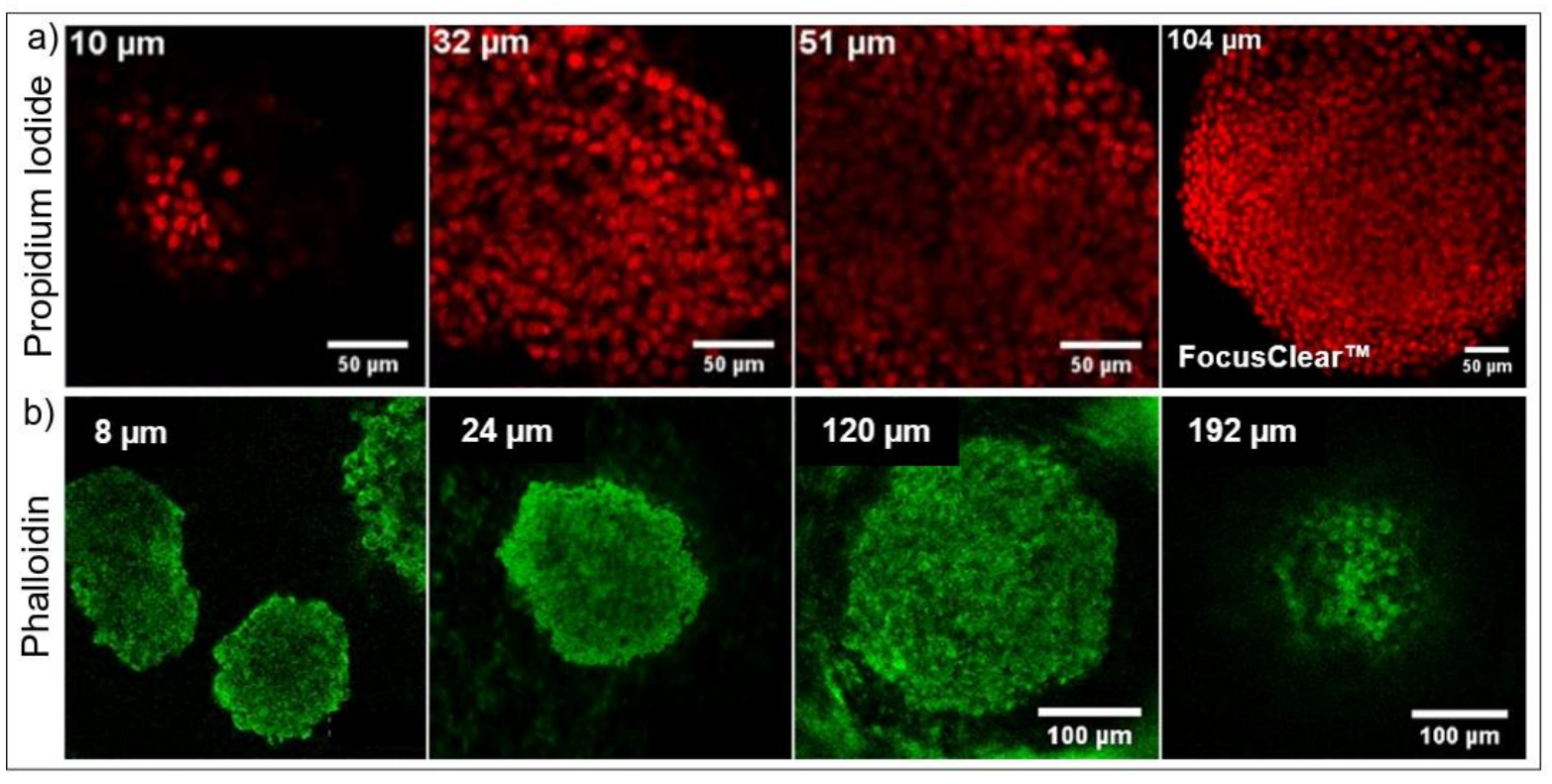

Figure 3-5 a) Cancer mono-culture spheroid stained with Propidium Iodide (nuclear stain). Image taken at different heights, as indicated on the different frames. In the last frame, spheroid in Focus Clear reveals a clear image of the nuclei in the center. b) Cancer mono-culture spheroid stained with phalloidin (F-actin staining) in Focus Clear. Images taken at different sections of the spheroid, 


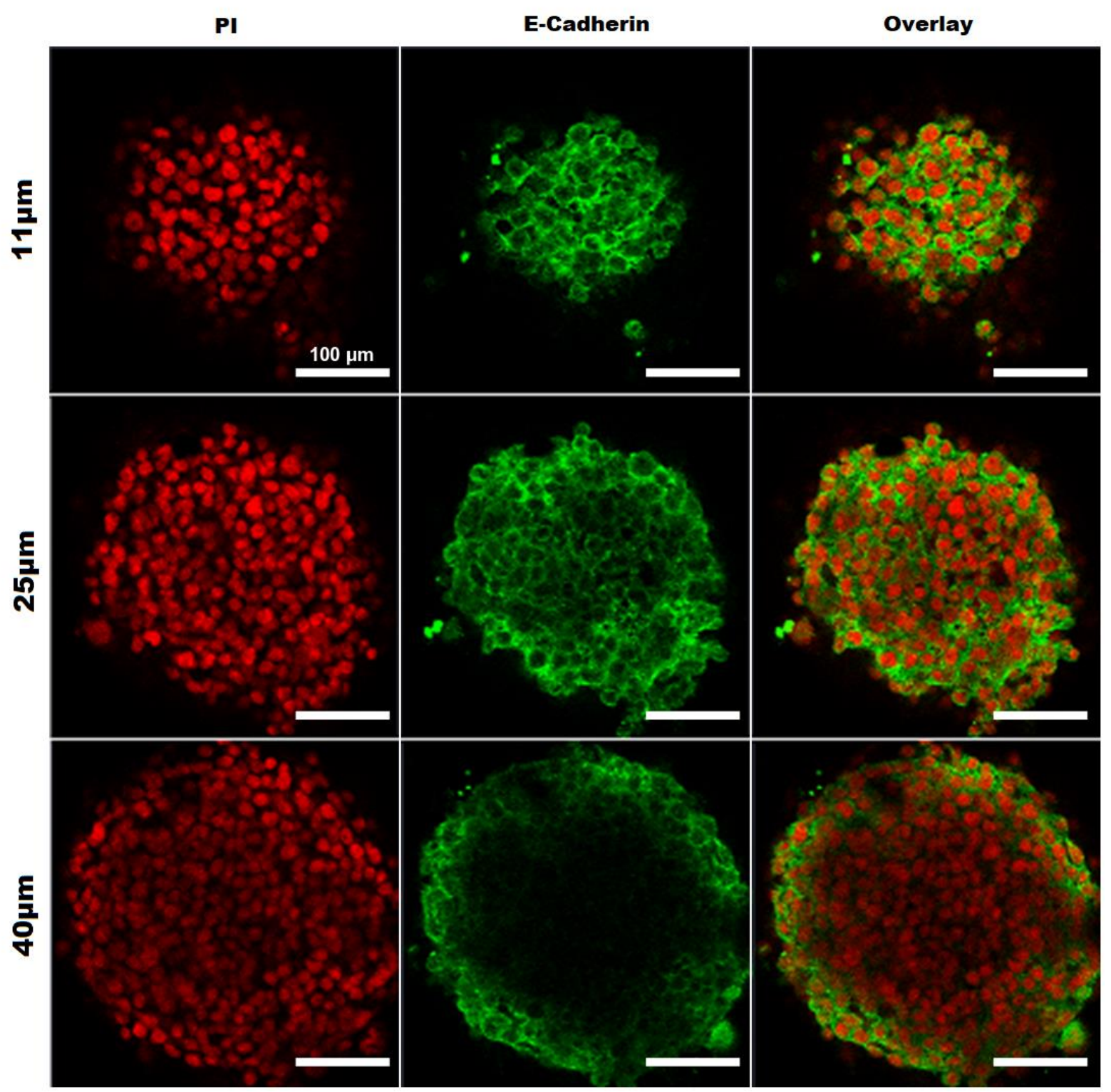

Figure 3-6 Cleared 4 T1 cancer mono-culture spheroid stained with E-cadherin (E-cad) and propidium iodide (PI), in Focus Clear. Images taken at different heights revealing a rapid decrease in intensity of the E-Cadherin signal already at 40 $\mu \mathrm{m}$, and indicating a limited antibody penetration.

In a second experiment, phalloidin was replaced with antibodies, specifically against E-cadherin and $\alpha$-smooth muscle actin ( $\alpha$ SMA), to evaluate antibody staining after clearing. E-cadherin mediates cell-cell adhesion, and after staining, the fluorescent signal should only be observed at the cellular junctions. $\alpha \mathrm{SMA}$ is a cytoplasmic protein found primarily in fibroblasts and smooth muscle cells. By using these two antibodies in tumour spheroids composed of cancer cells and fibroblasts, we aimed to distinguish both cell types in the tissue. In the uncleared tissues, both E-cadherin and $\alpha$ SMA were specifically stained, but as previously 
observed, no staining could be seen in the core (Figure 3-4.b). After clearing, E-cadherin staining was no longer limited to the cell membrane, suggesting a decrease in the specificity of the antibody binding. Moreover, both antibody staining agents only stayed at the periphery of the tissue while the PI counterstaining penetrated throughout the entire tissue (Figure 3-6). This difference in penetration could be explained by the difference in size between the antibodies (molecular weight of anti-E-cadherin $97 \mathrm{kDa}$, anti- $\alpha \mathrm{SMA} 42 \mathrm{kDa}$, secondary antibody 110 $\mathrm{kDa}$ ) and the small molecules (molecular weight of PI $0.67 \mathrm{kDa}$, Phalloidin $0.79 \mathrm{kDa}$ ), which significantly affects their diffusion rate. This effect is reinforced compared to standard immunostaining protocol as the polymer generated from the acrylamide and bis-acrylamide monomers has a pore size between 3 and $30 \mu \mathrm{m}$, depending on the hydrogel composition, as reported by Yang et al. [9]. An attempt to increase the penetration depth of the antibodies was performed by doubling the incubation time, yet only with a very small improvement (data not shown).

\subsubsection{Dynamic perfusion}

\subsubsection{Clearing}

To promote the penetration of the antibodies in the tissue, it was decided to adapt a different incubation strategy by implementing active perfusion instead of passive diffusion, which would also allow decreasing the protocol time. Recently, Chen et al. [6] notably reported that using active perfusion would enhance the efficiency of the clearing process (Figure 3-7.a).

a)

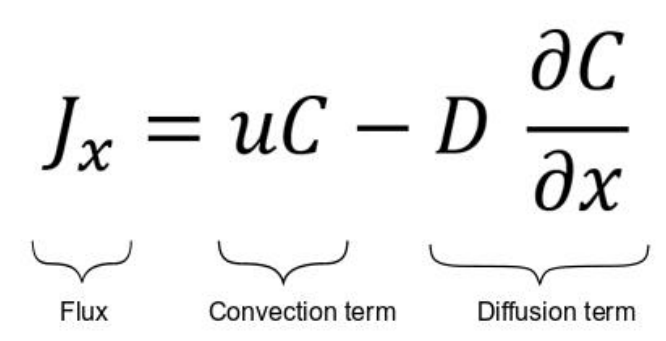

b)

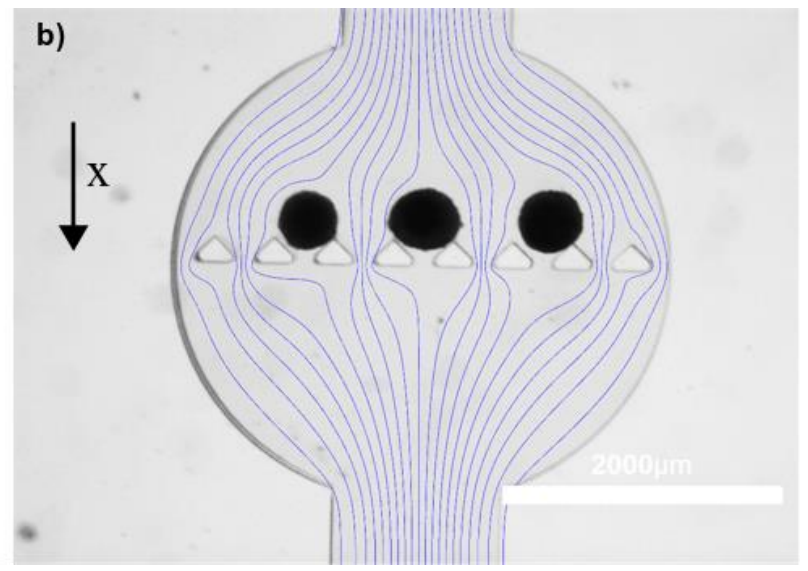

Figure 3-7 a) $1 D$ Equation of Convection-Diffusion in the direction of the flow $(x)$, with $C$ the concentration of the considered species, $u$ the flow velocity, and $D$ the diffusion coefficient of the same molecular species b) Three spheroids (here fibroblast-cancer cell co-culture) trapped in a microfluidic device; streamlines are drawn in blue (as derived from COMSOL simulations). 
Here, after fixation with formaldehyde as before, the spheroids were pipetted into a microfluidic device ( $3-5$ spheroids per device) with a chamber containing an array of pillars to trap them, and therein exposed directly to flow (Figure 3-7.b).

To test whether the flow could damage the spheroids, a preliminary experiment was performed in the device by gradually increasing the flow-rate, starting at $10 \mu \mathrm{L} / \mathrm{h}$, while observing the fate of the spheroids. The spheroids shrank gradually under the pressure induced by the flow, until a flow of $5 \mathrm{~mL} / \mathrm{h}$, at which most of the spheroids would have shrunk to such an extent that they escaped the trapping structures, the distance between the pillars being 100 $\mu \mathrm{m}$. To test to which extent using a flow could influence the efficiency of each step of the clearing protocols, different flow-rates were selected, based on previous works by Chen et al and Grist et al. $[6,15]$ that used perfusion-based clearing conditions in microfluidic devices. Three values of flow-rate were retained, based on the dimensions of the devices in their work and ours, and the mean velocity employed in previous work. The selected flow-rate values were $60 \mu \mathrm{L} / \mathrm{h}$ for the embedding and polymerization steps, and $800 \mu \mathrm{L} / \mathrm{h}$ and/or $2820 \mu \mathrm{L} / \mathrm{h}$ for perfusing the clearing solution.

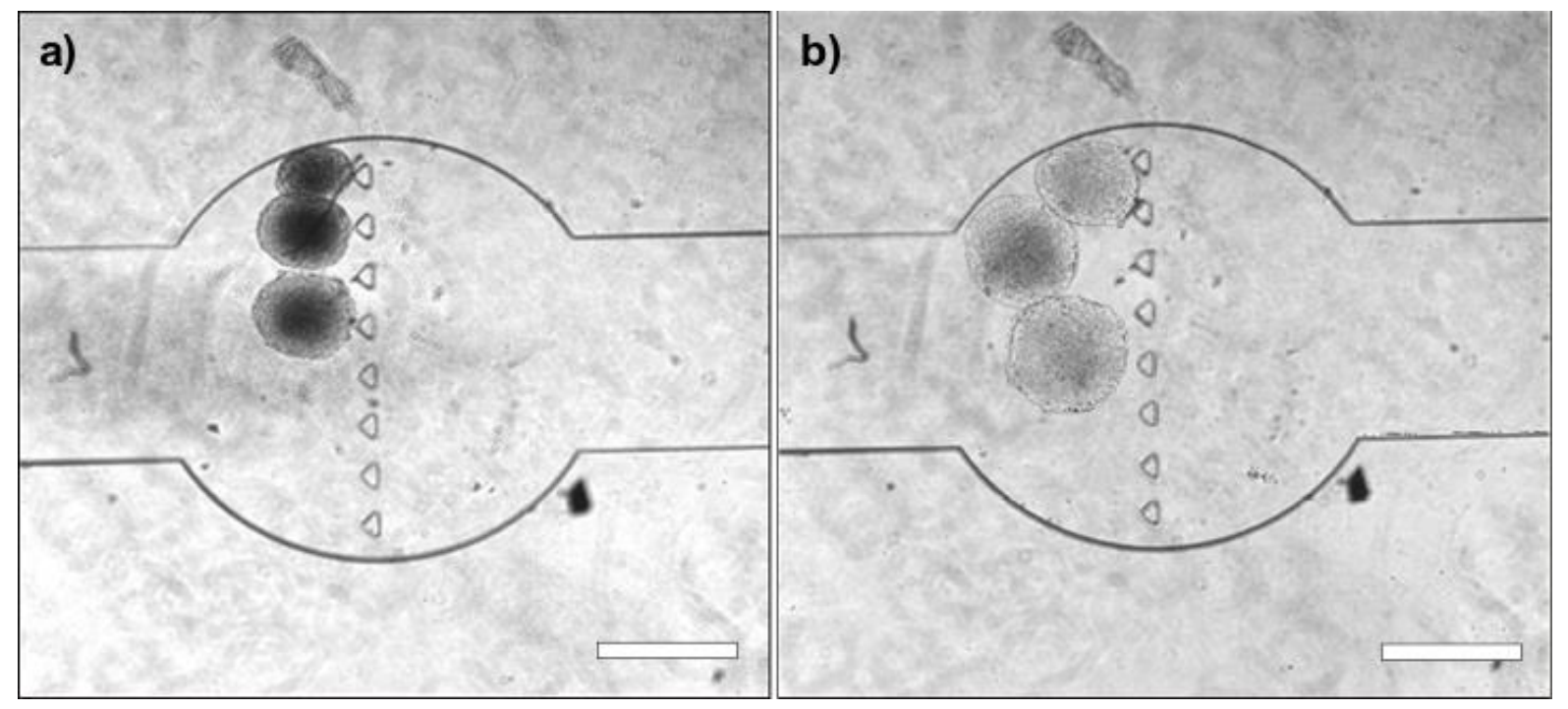

Figure 3-8 3T3:4T1 5:1 co-culture spheroids in a microfluidic device a) before and b) after $20 \mathrm{~min}$ clearing with $8 \%$ SDS at $800 \mu \mathrm{L} / \mathrm{h}$. The spheroids became transparent, and swelled as a result of the osmotic pressure difference. Scale bar $=1 \mathrm{~mm}$

Embedding and polymerization of the hydrogel were performed in the device. The embedding solution penetrated completely in the spheroids within $30 \mathrm{~min}$ at $60 \mu \mathrm{L} / \mathrm{h}$, and the acrylamide hydrogel polymerization was typically completed within $2 \mathrm{~h}$ (at $37^{\circ} \mathrm{C}$ ) in the device. The clearing solution $\left(8 \% \mathrm{SDS}\right.$ at $37^{\circ} \mathrm{C}$, as previously described) was then perfused at 800 
$\mu \mathrm{L} / \mathrm{h}$ and a time-lapse of the tissue clearing process was recorded (Figure 3-8). This time-lapse indicated, as previously, a rapid swelling of the spheroids, almost immediately after the start of experiment, continuing for 15-20 min, to reach in average a total swelling of the spheroids of about 25\%, depending on the cellular composition (Figure 3-S1.c). This swelling was accompanied by a rapid clearing of the tissue, which can be explained by a decrease in the tissue density. After swelling, the tissue transparency slowly increased for approximately 40 min, after which no difference in the brightness inside in the tissues could be noticed. The spheroids still did not appear completely transparent as their core would remain opaque, but this could be explained by a mismatch in refractive index as discussed before, which is addressed at a later stage by adding the refractive index matching solution Focus Clear.

When the same experiment was repeated at a flow rate of $2820 \mu \mathrm{L} / \mathrm{min}$, a comparable clearing speed was found, but the spheroids did not swell as much, most likely because the use of a high flow-rate introduces a counterbalancing effect by inducing tissue shrinking, as discussed previously.

\subsubsection{Hoechst Staining}

To assess the influence of the flow on the penetration of the fluorescent probes, the small probe Hoechst was first injected at $800 \mu \mathrm{L} / \mathrm{min}$ and/or $2820 \mu \mathrm{L} / \mathrm{min}$, and pictures acquired using confocal microscopy at different time points.

After $20 \mathrm{~min}$ perfusion at $800 \mu \mathrm{L} / \mathrm{h}$, the small $4 \mathrm{~T} 1$ mono-culture spheroids were completely stained, but the 3T3:4T1 5:1 co-culture spheroids still presented a dark core (Figure 3-8.a). After $40 \mathrm{~min}$ incubation, both spheroid types were completely stained. For larger spheroids (diameter $>500 \mu \mathrm{m}$ ) however, prolonged exposure to the staining agent was necessary. After adding $2 \mathrm{~h}$ of passive incubation (no flow), Hoechst penetrated at $90 \%$ in the spheroids, and after $4 \mathrm{~h}$ spheroids were completely stained (Figure 3-8.c). These results indicate that the use of perfusion actively promoted the penetration in the outer layer of the spheroids, but remained ineffective to stain their core. This phenomenon could be explained by the permeability and porosity properties of the hydrogel, which would govern the penetration rate of Hoechst as it reached deeper into the tissues. For this reason, only short times of perfusion were used, followed by long passive incubation periods. After pre-fixation, the total time needed to achieve complete embedding, clearing and staining was still significantly faster than previously described for passive clearing approach (4 h 20 min instead of $24 \mathrm{~h}$ ). Chen et al., 
completed their own complete protocol of fixation, embedding, clearing and staining in under $5 \mathrm{~h}$, on spheroid ranging from 250 to $450 \mu \mathrm{m}$ [6].

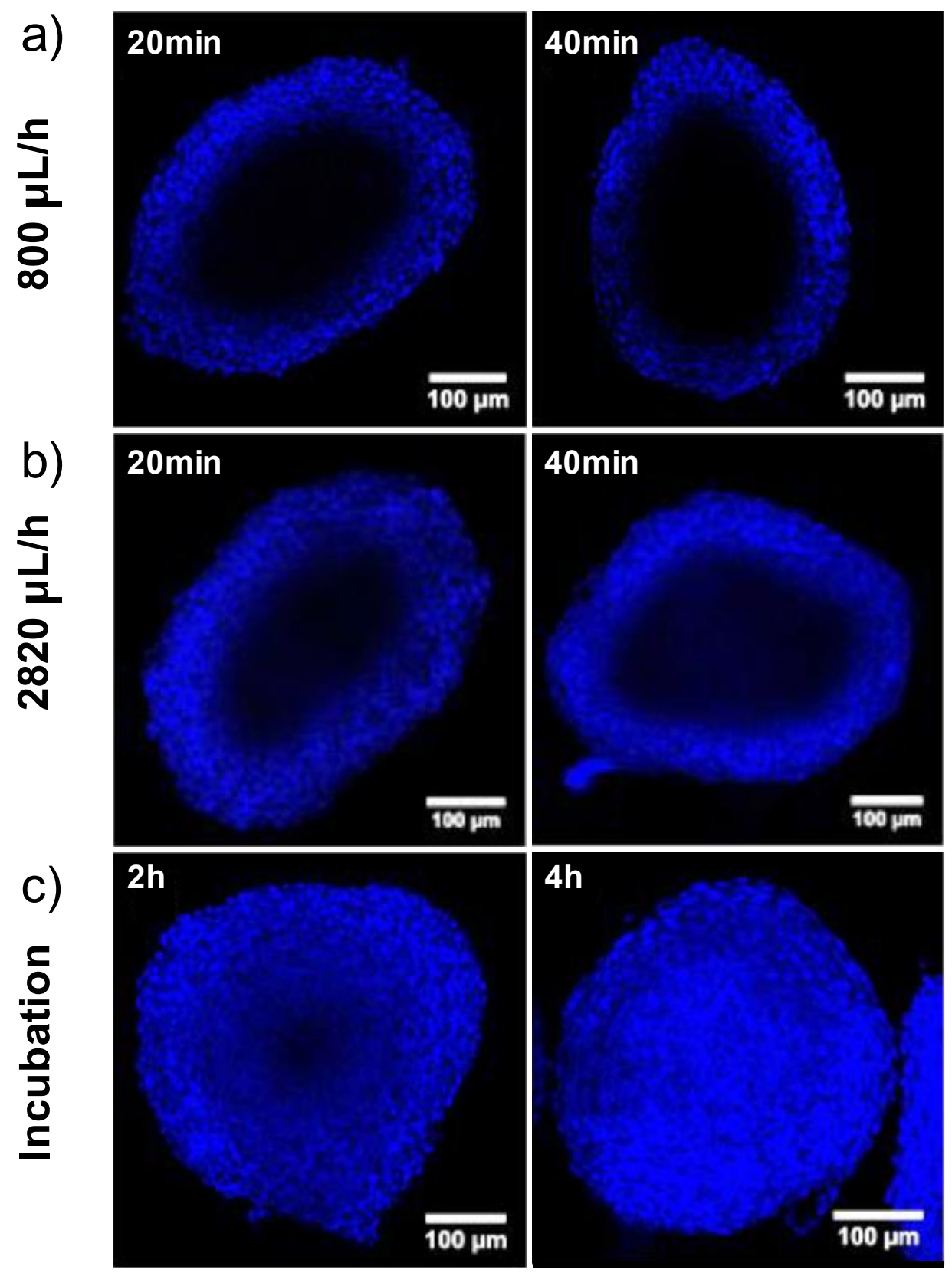

Figure 3-9 Cleared co-culture spheroid stained with Hoechst at a) $800 \mu \mathrm{L} / \mathrm{h}$ and b)2820 $\mathrm{\mu L/h}$. Incomplete staining of the tissue with 40 min perfusion, with no significant improvement with higher flow rate. By complementing the $800 \mu L / h$ perfusion with $2 h$ and $4 h$ (c) passive incubation, complete uniform staining was obtained.

When perfusing Hoechst at $2820 \mu \mathrm{L} / \mathrm{min}, 40$ min was necessary to fully stain the small 4T1 spheroids, and even more time (data not shown) to stain the 5:1 ones (Figure 3-9.b). It appeared that increasing the flow rate did not improve the penetration of Hoechst, but rather decreased it. This unexpected reverse in penetration efficiency could be explained again by the 
counter-acting effect of the higher flow rate that induced shrinking of the spheroids, increasing thereby the tissue density and therefore slowing down the penetration of the staining agents.

\subsubsection{Immunostaining}

Based on the initial results obtained for the small probe Hoechst, it was decided to perfuse antibodies for $30 \mathrm{~min}$ at either $800 \mu \mathrm{L} / \mathrm{min}$ or $2820 \mu \mathrm{L} / \mathrm{min}$, followed by a static incubation overnight. First, E-cadherin antibody was tested. At a flow rate of $800 \mu \mathrm{L} / \mathrm{h}$, measuring the fluorescent signal using confocal microscopy after overnight incubation revealed a limited penetration of the antibodies inside the spheroids: the average penetration of E-cadherin was $65 \pm 15 \mu \mathrm{m}$ in $4 \mathrm{~T} 1$ mono-culture $(\sim 160 \mu \mathrm{m}$ radius $)$ spheroids and $55 \pm 15 \mu \mathrm{m}$ in 5:1 co-culture spheroids ( $260 \mu \mathrm{m}$ radius), and disappointingly showed no significant increase in penetration compared to the previously described static incubation. Additionally, the Ecadherin immunostaining was expected to only stain the cell membranes, but in the experiment, a fluorescent signal could be found everywhere in the cell, indicating non-specific staining (Figure 3-10.a). When perfusing the antibody solution at $2820 \mu \mathrm{L} / \mathrm{h}$, the penetration depth became $50 \pm 10 \mu \mathrm{m}$ for the $4 \mathrm{~T} 1$ mono-culture spheroids, and $25 \pm 5 \mu \mathrm{m}$ in the co-culture spheroids (Figure 3-10.b), revealing in average a decrease of the penetration depth when using dynamic perfusion instead of passive incubation. Similar results were obtained with other antibodies for aSMA and Ki-67 (Figures 3-S3 and S4).

\subsubsection{Elucidation of the antibody non-specific binding}

We hypothesized that the non-specific binding could arise from two possible phenomena, both inducing a loss of the epitope on the antigen, so that the latter could be not recognized by the antibody. First, cross-linking of the proteins by the formaldehyde into the hydrogel matrix could block the epitope and prevent its interaction with the antibody used for tissue staining. Second, SDS, used here to remove lipids from the tissues in the form of micelles, is also widely used as a denaturating agent for proteins. In other words, SDS can cause proteins to lose their 3D configuration, which could again result in the loss of the epitope on the antigen.

To test this hypothesis and determine the cause of the unspecific binding, monolayers and spheroids were stained with phalloidin after the different steps of the clearing protocol (fixation, embedding/polymerization, clearing), and by varying the concentrations of the 
a)

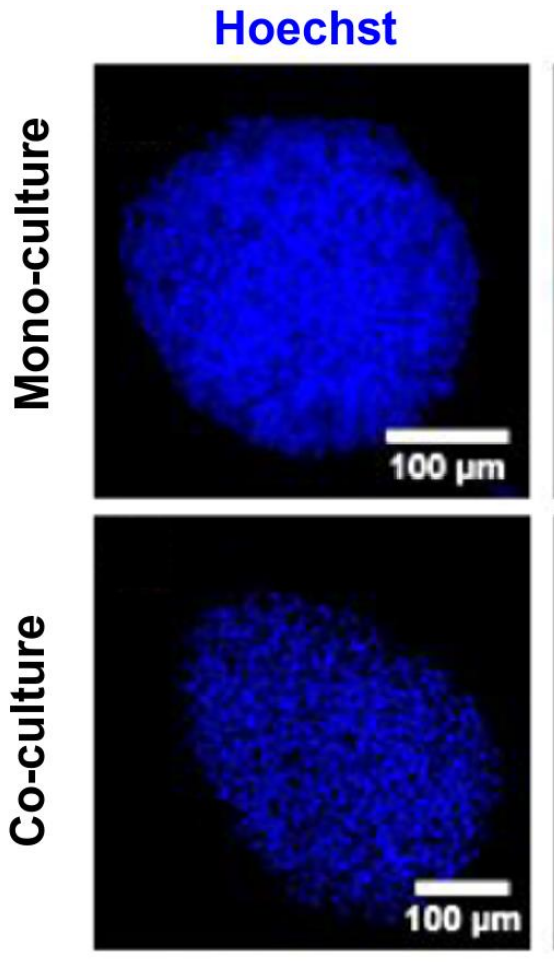

E-Cadherin
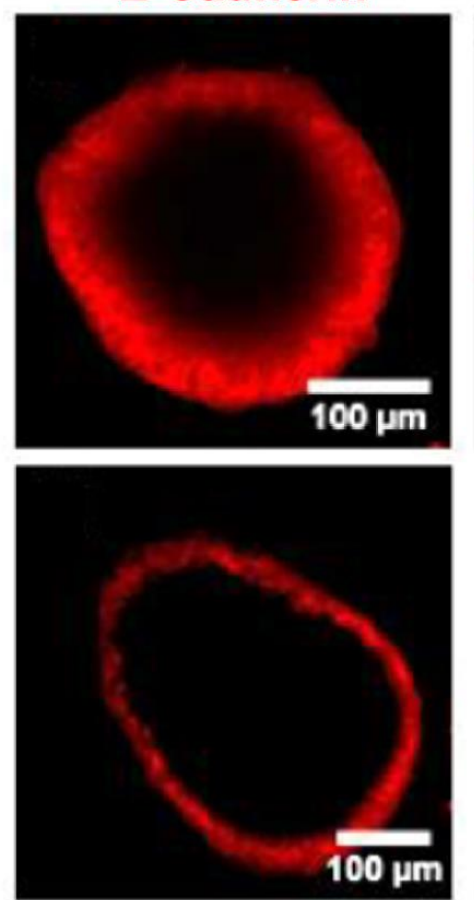
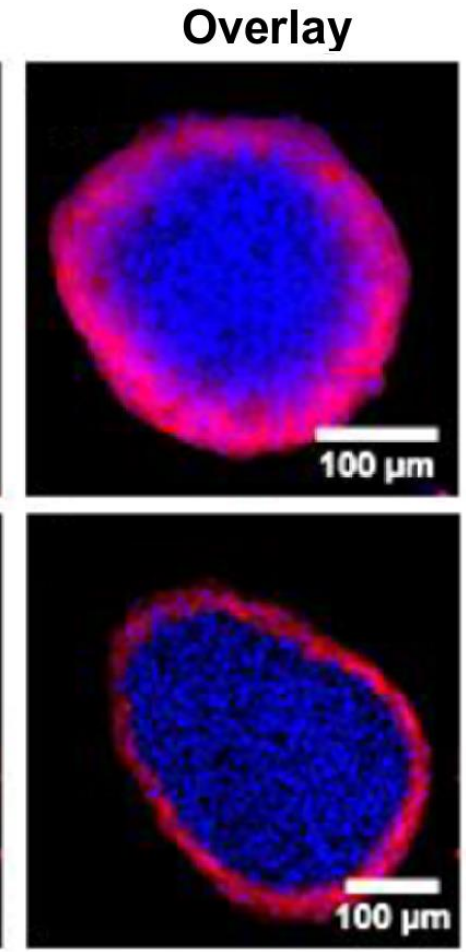
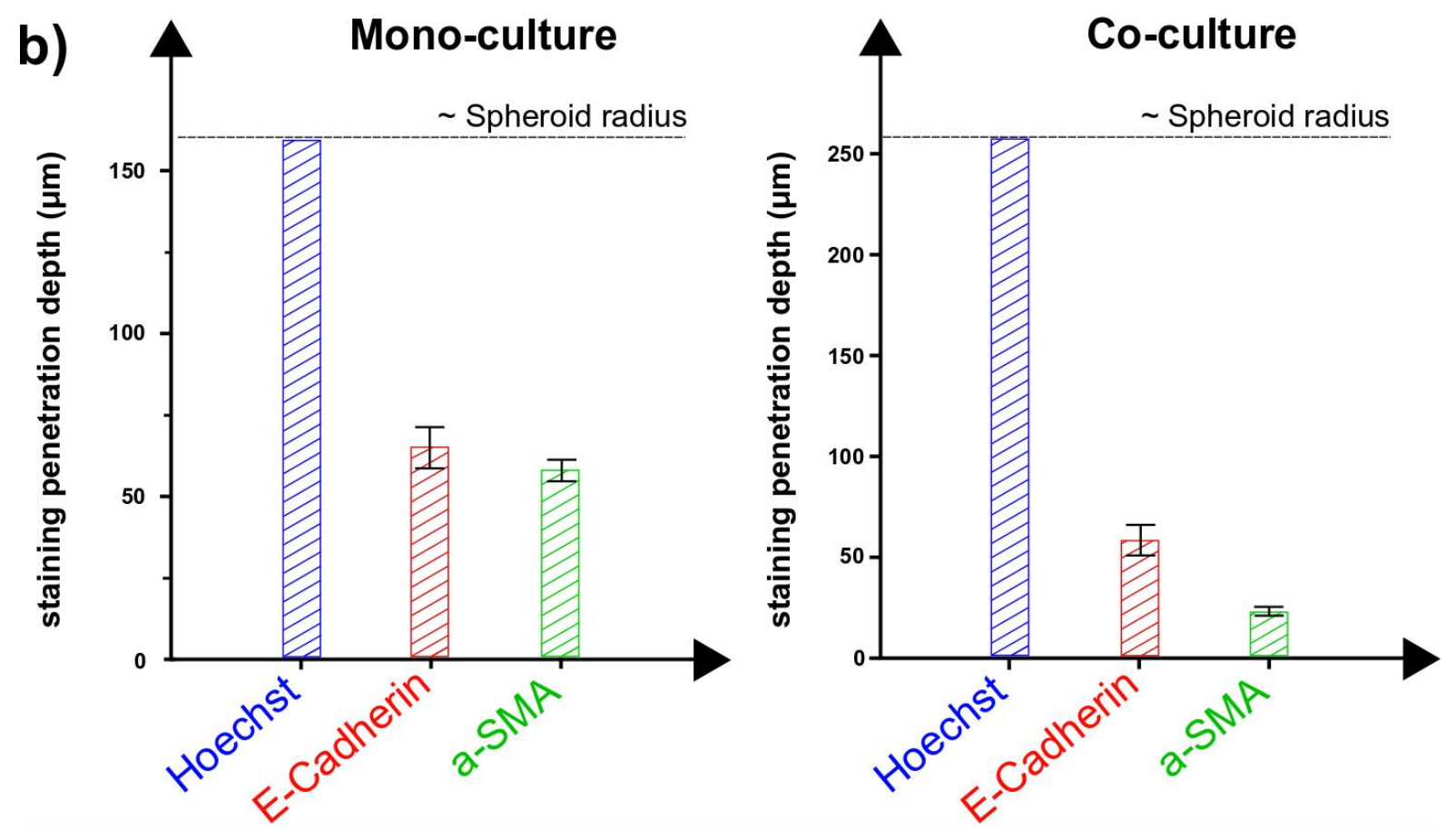

Figure 3-10 a) Confocal microscopy of 4T1 mono-culture spheroids and 5:1 co-culture spheroids, stained with Hoechst (blue, left column) and anti E-cadherin antibody (red, middle column). Primary antibodies, secondary antibodies and Hoechst were all infused for $30 \mathrm{~min}$ at $2820 \mu \mathrm{l} / \mathrm{h}$, followed by incubation overnight. Overlays are presented in the right column. b) Quantitative measurement of the penetration depth of Hoechst, and E-cadherin and $\alpha$-SMA antibodies in the spheroids. 
different chemicals (formaldehyde, PBS-Triton X100 (PBST), acrylamide, SDS) to identify when specificity would be lost.

Imaging of the monolayers fixed with $4 \%$ formaldehyde and washed with $0.1 \%$ PBST indicated proper binding of the phalloidin (Figure 3-S5.b)., and increasing the concentration up to $10 \%$ did not affect the staining (Figure appendix 3-S5.c.d). Embedding of the monolayer in the hydrogel matrix did not affect neither the binding affinity (Figure 3-10 a,b). After incubation overnight in the SDS-based clearing solution, actin filaments were no longer specifically stained and imaged, suggesting that either SDS or boric acid would be responsible for the non-specific binding (Figure 3-11.c,d). Boric acid was considered inert here as the $\mathrm{pH}$ was maintained at 8.5. According to literature, SDS can induce partial denaturing of the antigens and loss of the epitope recognized by the antibodies, and therefore was assumed be the cause of the non-specific antibody binding here [16].
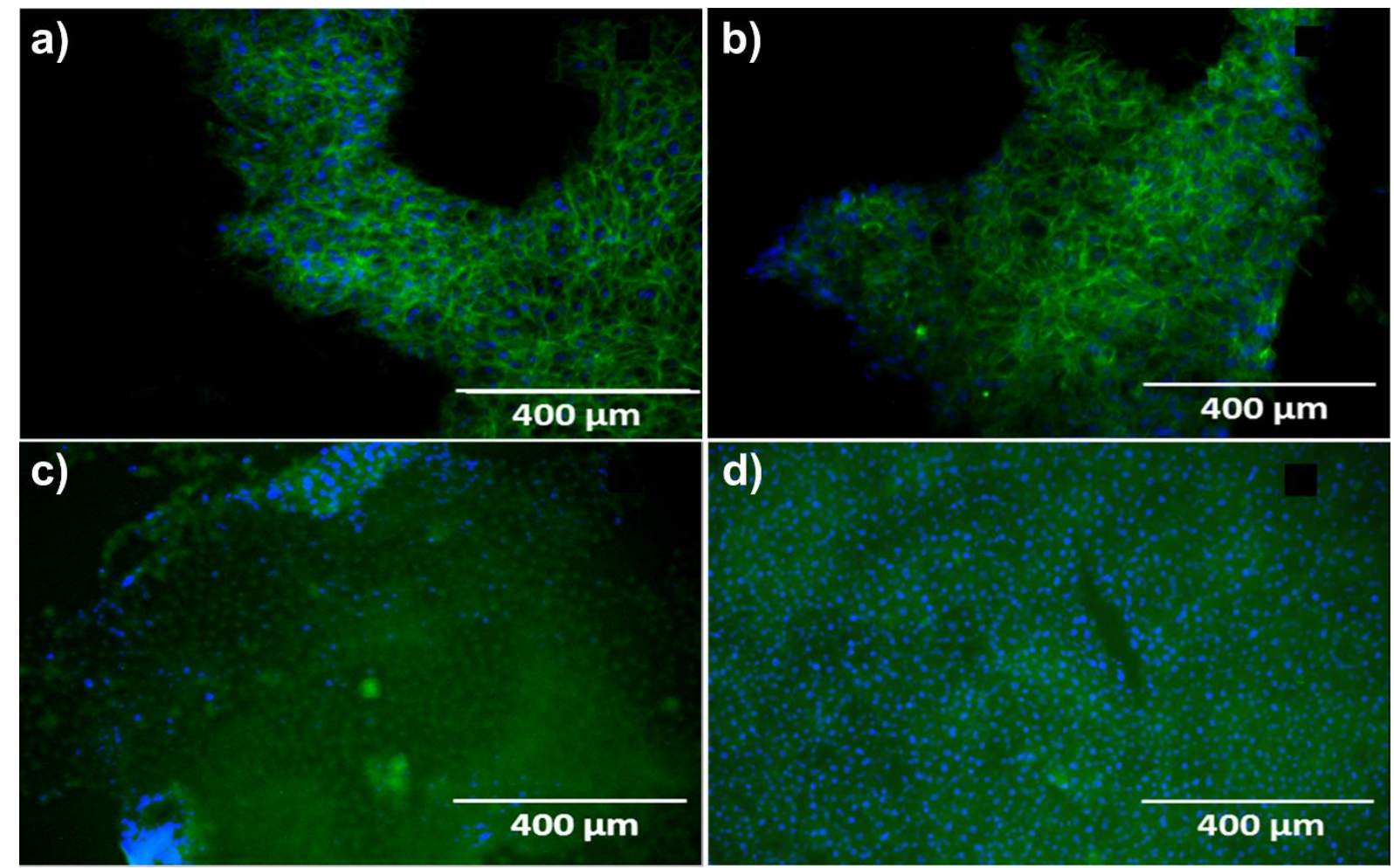

Figure 3-11 a,b) Imaging of phalloidin (green) and Hoechst (blue) of a 373 monolayer (a) and a 4 T1 monolayer (b), fixed in formaldehyde, washed with $0.1 \%$ PBST and embedded/polymerized with an acrylamide solution. c,d) Imaging of phalloidin (green) and Hoechst (blue) of a $3 T 3$ monolayer (c) and a 4 T1 monolayer (d), fixed in formaldehyde, washed with $0.1 \%$ PBST, embedded/polymerized with an acrylamide solution, and cleared with a 8\% SDS solution. 


\section{Conclusion}

Using the CLARITY and PACT protocols as references for our work, we successfully cleared multicellular tumour spheroids of different sizes and cellular compositions. By implementing our clearing protocol inside a microfluidic device and using a perfusion-based dynamic protocol, we achieved complete transparency of multiple tissues in a few hours instead of days when using a static diffusion-based approach. We demonstrated successful staining of all nuclei in the spheroids using a small fluorescent probe (Hoechst) using a combination of active perfusion and passive incubation, as revealed by confocal microscopy imaging of the samples.

However, when performing immunostaining (staining using antibody probes), we only achieved partial staining of the tissues, which was limited to the periphery even when using flow, the specific penetration depending on the exact tissue cellular composition. Our results and the literature would suggest that the pores in the hydrogel were too small for the penetration and diffusion of the antibodies toward the core of the spheroids. In future work, we will investigate alternative acrylamide formulations to generate a more porous polymer, allowing a better immunostaining of our samples.

Furthermore, for all antibody staining tested here, we noticed a strong non-specific binding, which was attributed to a partial loss of the antigen 3D structure induced by sodium dodecyl sulphate (SDS) that acted as a denaturating agent. In future work, we will use an alternative detergent to SDS to prevent this denaturation and/or find a solution to protect the epitope of the proteins prior to exposition to the detergent.

\section{ACKNOWLEDGMENT}

This work has been strongly supported by the active participation of several Bachelor Students. I would especially like to thank Martijn Kern, Janine Santing, and Marieke Meteling, from the University of Twente; Jerilaine Gomez, and Dzeneta Mahmutovic from the university of Saxion; and Stanislas Helle form the university of Lille, for their work. 


\section{REFERENCES}

1. Riffle, S., et al., Linking hypoxia, DNA damage and proliferation in multicellular tumor spheroids. BMC Cancer, 2017. 17(1): p. 338.

2. Grimes, D.R., et al., A method for estimating the oxygen consumption rate in multicellular tumour spheroids. J R Soc Interface, 2014. 11(92): p. 20131124.

3. Ferguson, R. and V. Subramanian, Embryoid body arrays: Parallel cryosectioning of spheroid/embryoid body samples for medium through-put analysis. Stem Cell Res, 2018. 28: p. 125-130.

4. Slot, J.W. and H.J. Geuze, Cryosectioning and immunolabeling. Nat Protoc, 2007. 2(10): p. 2480-91.

5. Treweek, J.B., et al., Whole-body tissue stabilization and selective extractions via tissue-hydrogel hybrids for high-resolution intact circuit mapping and phenotyping. Nature Protocols, 2015. 10(11): p. 1860-1896.

6. Chen, Y.Y., et al., Clarifying intact 3D tissues on a microfluidic chip for highthroughput structural analysis. Proc Natl Acad Sci U S A, 2016. 113(52): p. 1491514920.

7. Chung, K., et al., Structural and molecular interrogation of intact biological systems. Nature, 2013. 497(7449): p. 332-7.

8. Lee, E., et al., ACT-PRESTO: Rapid and consistent tissue clearing and labeling method for 3-dimensional (3D) imaging. Scientific Reports, 2016. 6.

9. Yang, B., et al., Single-Cell Phenotyping within Transparent Intact Tissue through Whole-Body Clearing. Cell, 2014. 158(4): p. 945-958.

10. Sridhar, A., et al., Microstamped Petri dishes for scanning electrochemical microscopy analysis of arrays of microtissues. PLoS One, 2014. 9(4): p. e93618.

11. Tomer, R., et al., Advanced CLARITY for rapid and high-resolution imaging of intact tissues. Nat Protoc, 2014. 9(7): p. 1682-97.

12. Daster, S., et al., Induction of hypoxia and necrosis in multicellular tumor spheroids is associated with resistance to chemotherapy treatment. Oncotarget, 2017. 8(1): p. 17251736.

13. Gunther, S., et al., Polyphenols prevent cell shedding from mouse mammary cancer spheroids and inhibit cancer cell invasion in confrontation cultures derived from embryonic stem cells. Cancer Lett, 2007. 250(1): p. 25-35.

14. Stricker, J., T. Falzone, and M.L. Gardel, Mechanics of the F-actin cytoskeleton. J Biomech, 2010. 43(1): p. 9-14.

15. Grist, S.M., et al., On-chip clearing of arrays of 3-D cell cultures and micro-tissues. Biomicrofluidics, 2016. 10(4).

16. Lee, A., et al., Denaturation of Proteins by SDS and Tetraalkylammonium Dodecyl Sulfates. Langmuir, 2011. 27(18): p. 11560-11574. 


\section{APPENDIX}
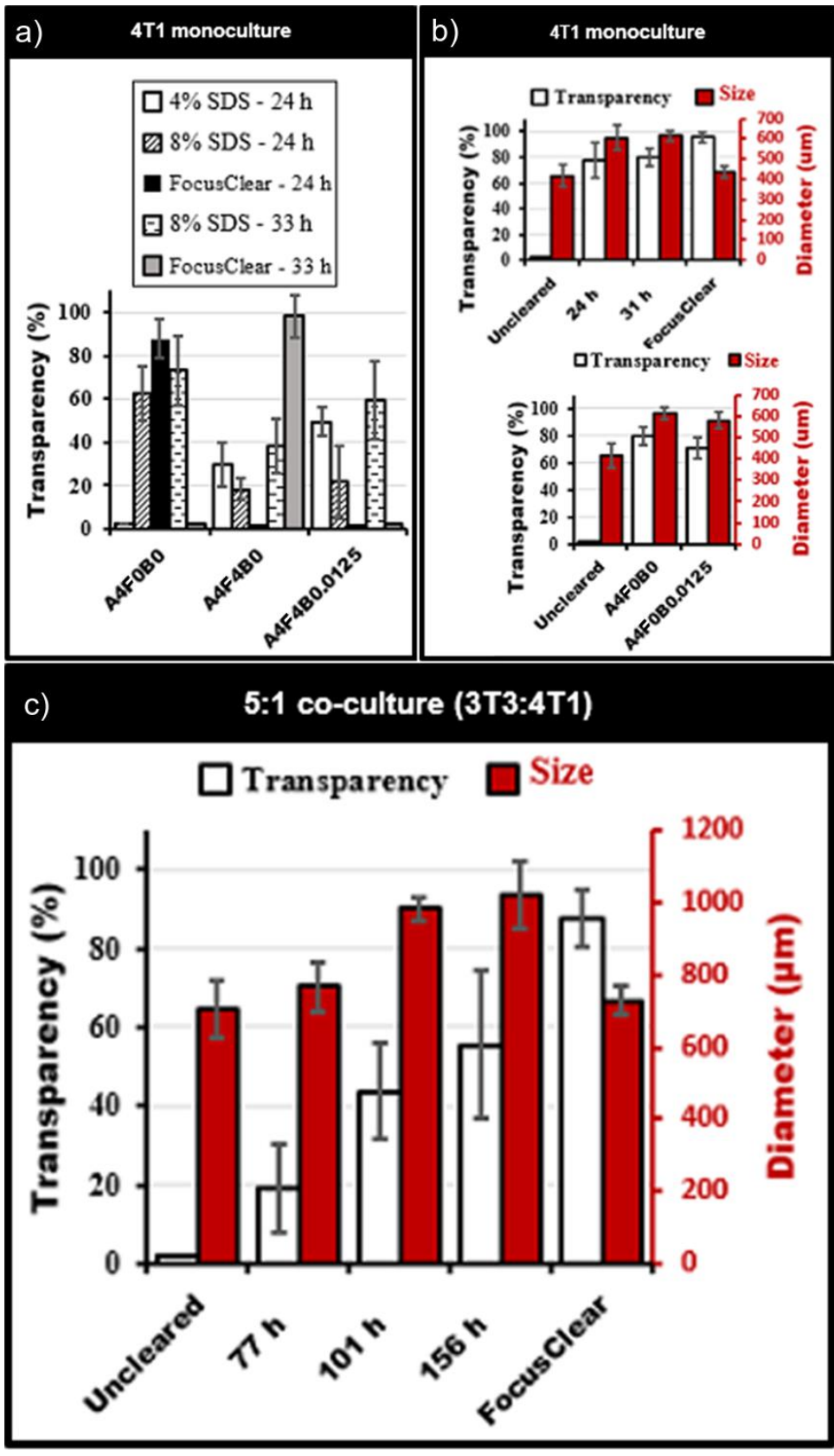

Figure 3-SI: Cleared spheroid characterization. Values are presented as mean \pm standard deviation. a) Comparison of hydrogel and clearing conditions in $4 T 1$ spheroids (300-400 $\mu \mathrm{m})$. Bars at $0 \%$ indicate missing data due to loss of tissue. Prefixation and reduction in cross-linking results in much faster tissue clearing. No significant difference in transparency between $4 \%$ and $8 \%$ SDS is seen, while the RI matching solution greatly increases transparency. b) Clearing time and cross-linking effect $4 T 1$ only spheroids. Top graph is for A4P0BO. As transparency increases the tissues swell by almost 60\%, while returning to initial size in FocusClear. The bottom graph shows the effect of increased bis-acrylamide: slower clearing and less swelling. c) Transparency and size of large 5:1 (3T3:4T1) co-culture spheroids over time. Tissues were pre-fixated and had an initial size of 600-800 $\mu \mathrm{m}$. Lipid removal takes much longer due the size of the tissues. It can be seen that they swell greatly, an almost 70\% increase in size after 156 hours. However, they shrink back to their original size in FocusClear. 

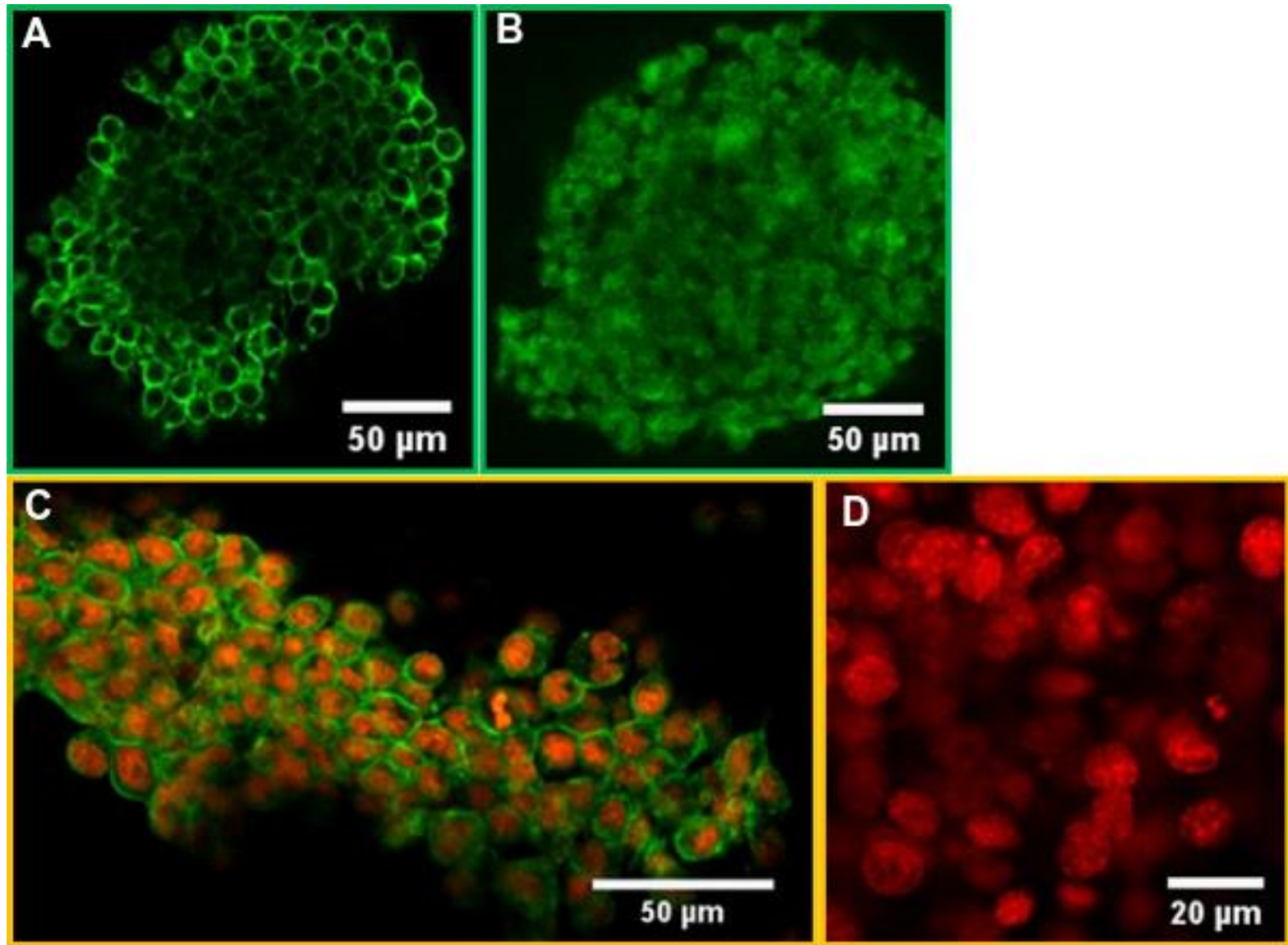

Figure 3-S2 Actin green troubleshooting (Spheroids image in PBS-TritonX). A) Uncleared 4 t1 only spheroid with specifiv actin staining. B) Uncleared $4 t 1$ only spheroid washed with SDS for 30min, decrementing the specificity of the actin staining. C) Uncleared co-culture spheroid stained with actin and propidium Iodide (PI). D) Core of a cleared co-culture spheroid with Actin and PI. PI is visible and clear, whereas Actin is absent. 


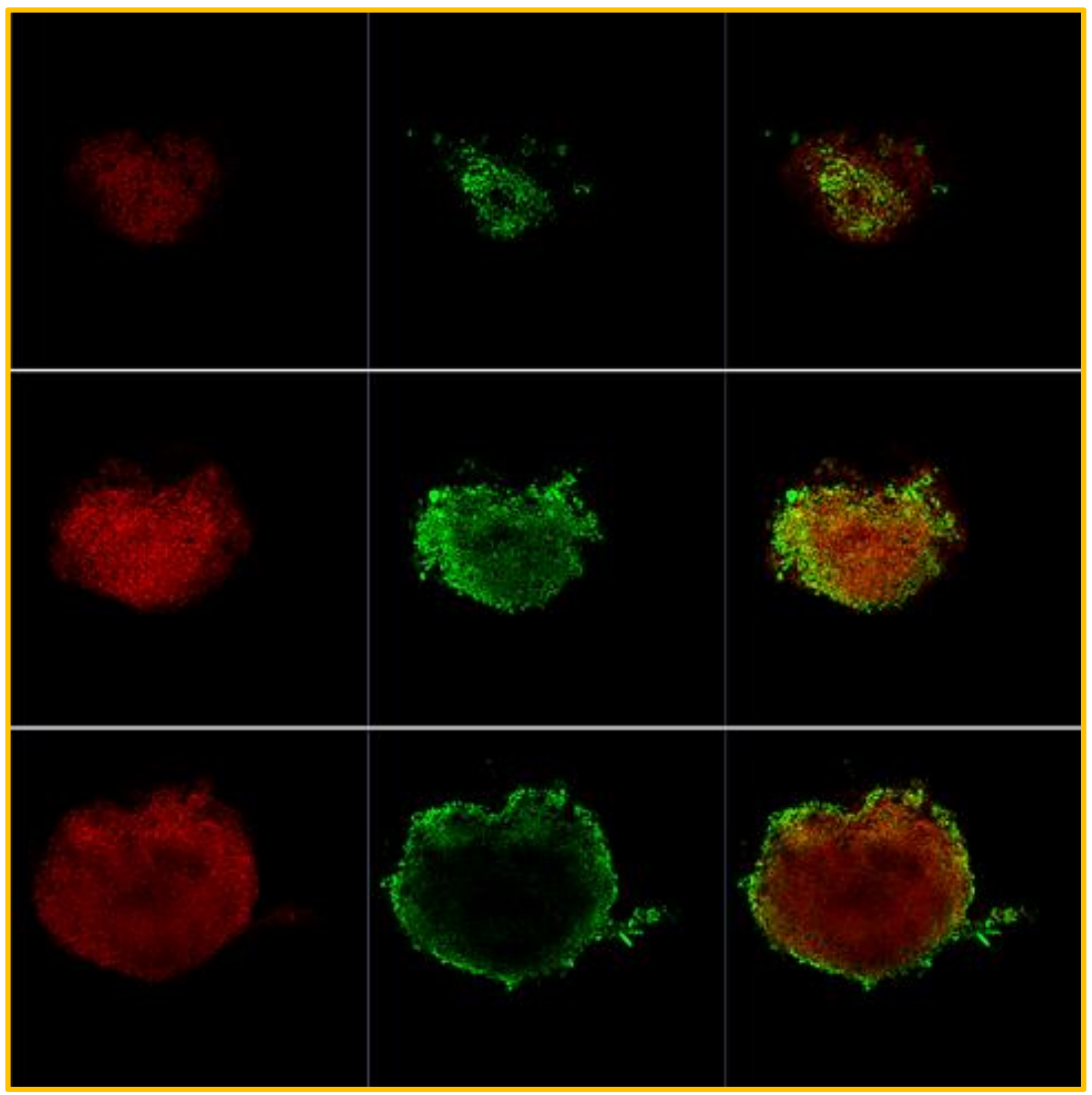

Figure 3-S3 Cellular organization of a large 5:1 (3T3:4T1) partially cleared co-cultured spheroid, stained with E-cadherin (Ecad) and propidium iodide (PI). Imaged in PBST. Numbers in the top-left indicate tissue depth. E-cadherin loses intensity much faster than PI. Only edges of the tissues are visible and brighter, which could indicate 4T1 is localized on the outside of the tissue. This pattern could also be caused by light scattering, so nothing can reliably be concluded from these images. 

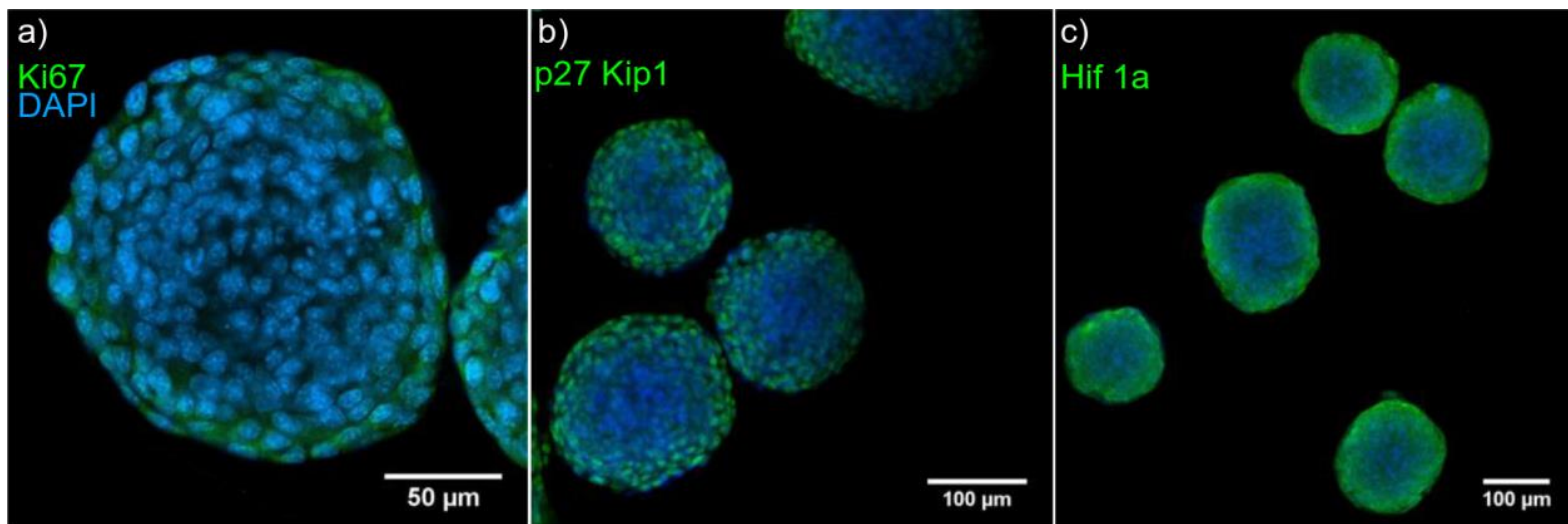

Figure 3-S4 Confocal Imaging of cleared4t1 only spheroids, stained with a) Ki67, b)p27 Kip1, or c)Hif 1 $\alpha$ antibody conjugated with a green fluorophore, and Hoechst in blue, used as counterstain. Clearing resulted in unspecific binding, Ki67 and p27 kipl antibody supposedly targeting the cell nuclei only.

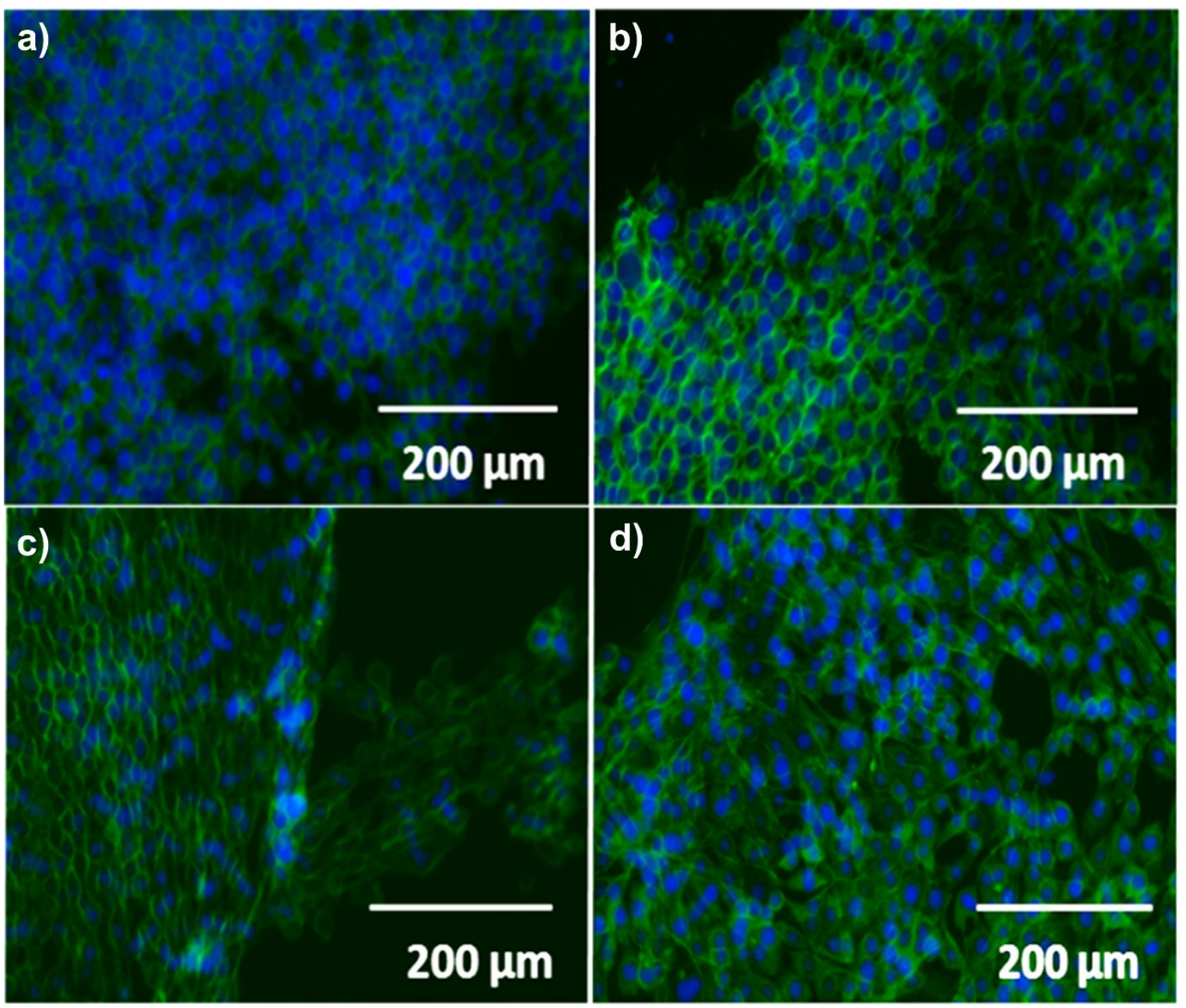

Figure 3-S5 Imaging of phalloidin (green) and Hoechst (blue) on $3 T 3$ monolayers, fixed with 4\% formaldehyde, and washed with a) $0 \%$ (control), b) $0.1 \%$, c) $1 \%$, and d) $10 \%$ PBS-Triton X100. 


\section{Chapter 4- Molecular and Cellular organization of the 3D Tumour Spheroid}

\section{Abstract}

To characterize the location of proteins of interest, we tested in the previous chapter a method to clear the tissue, which showed significant limitations for immunostaining. Here we explored a different approach for the same purpose; immunolabeling-enabled imaging of solvent-cleared organs (iDISCO), followed by HISTO-M mounting solution developed by the company VISIKOL ${ }^{\mathrm{TM}}$. We first investigated the shape of multicellular tumour spheroids (MCTS), prepared from murine cancer cells (4T1) with or without murine cancer fibroblasts (3T3), by using different well array geometrics (flat bottom vs. round bottom microwells), to generate the spheroids, and in turn, to obtain isotropic gradients of oxygen and nutrients. We generated MCTS of $c a .500 \mu \mathrm{m}$ in diameter. We next successfully cleared the tissues, which was accompanied with the expected shrinkage of the tissue. We next performed specific immunostaining, targeting different markers related to the position of the cells in the spheroids (periphery vs. core). We observed a decreasing amount of proliferative cells overtime, concentering toward the outer rim. We showed a strong expression of Hypoxia Induced Factor 1- $\alpha$ (HIF-1 $\alpha$ ), promotor of apoptosis, throughout the tissue. However, when targeted, we found very few apoptotic cells in the spheroids, which concurs with the knowledge that cancer cells evade the programmed cell death. Finally, we investigated the production of extracellular matrix (ECM) by fibroblasts, and noticed an increase in ECM overtime, starting from the outer layer of the MCTS at day 3, and reaching its core after 10 days in culture. 


\section{INTRODUCTION}

In the previous chapter, we established a protocol to render tissues transparent to notably support the imaging of 3D intact MCTS using a method based on CLARITY (Clear Lipidexchanged Anatomically Rigid Imaging/immunostaining-compatible Tissue hYdrogel) and PACT (Passive CLARITY Technique). We successfully rendered the tissues transparent, but, in our hands, the process compromised the protein 3D conformation and prevented accurate immunostaining, since immunostaining relies on antibody-antigen interactions, for which the protein 3D configuration is key. CLARITY, first proposed by Chung et al. in 2013, was implemented to render tissues transparent, by removing the lipids and creating another framework to hold all biomolecules in place $[1,2]$. However, two other methods have been developed to clear tissues, iDISCO and SeeDB, using different strategies and reaching similar results, as reviewed by A. Azaripour et al. [3].

The first clearing method was developed by Spalteholz in 1911, when investigating the differences in refractive index between tissues. By using a combination of methylsalycylate, benzyl benzoate (BB) and wintergreen oil, Spalteholz was able to clear brain tissues; yet the process was accompanied by undesired side effects like tissue shrinkage, bubble formation and tissue necrosis. This method was later improved by Dodt. et al., by mixing benzyl alcohol with benzyl benzoate (BA-BB) to pre-dehydrate the tissue samples, and mounting them in dibenzylether (DBE) to reduce any background fluorescence and therefore enhance specific fluorescence signals [4]. This better protocol, also known as iDISCO (immunolabeling-enabled imaging of solvent-cleared organs), has become a standard for tissue clearing [5]. The main limitation of this method however, remains the significant shrinkage of the tissue induced by the lipid removal, which is not compensated, as CLARITY does, by creating a polymer hydrogel matrix.

The third method, "Scale", differs from the other two by clearing the tissues using a hydrophilic solution, eliminating the need for a dehydration step [6]. By applying a mixture of urea and glycerol, the tissues were cleared while the fluorescence signals were preserved, and fluorescently-labelled antibodies against biomarkers could be applied. The main drawback of Scale is the fragility of the resulting samples. To circumvent this, the clearing solution has been replaced by organic solvents (e.g., Fructose). This new method, called SeeDB, achieves tissue 
clearing, without any swelling or shrinkage effect, and does not cause any morphological change [7]. It is however limited to tissues of small size $(<1 \mathrm{~mm})$, has a long clearing step $(\sim 3$ days), and does not promote the penetration of macromolecules as antibodies.

More recently, inspired by the iDISCO protocol, the company VISIKOL ${ }^{\mathrm{TM}}$ proposed a new patented mounting solution, VISIKOL HISTO-M, to replace the DBE mounting solution, specifically for clearing of small tissue samples such as spheroids. Originally proposed as an alternative to chloral hydrate, a common clearing agent for plant tissues, this solution has been optimized for a variety of animal tissues [8]. VISIKOL ${ }^{\mathrm{TM}}$ offers a relatively simple and fast protocol with a lot of potential to provide information deep within the tissue [9].

In this chapter, we tested an alternative strategy to the CLARITY method used in chapter 2. Here, in the view of using immunolabelling for our tumour spheroids, we applied the iDISCO protocol, combined with the VISIKOL HISTO-M mounting solution. Using this method, we set ourselves to characterize the different cell phenotypes associated with the presence of gradients of oxygen and nutrients in our samples [10,11], as discussed in previous chapters. Additionally, we investigated the production of Extracellular Matrix (ECM) inside the spheroids. Indeed, as previously described, the ECM, which is produced by stromal cells, and specifically fibroblasts here, plays an important role as a "mortar" for the tumour tissue [12]. It can also affect the tissue density and, in time, the aforementioned gradients, as well as the penetration of drugs. Yet, we have not identified where inside the spheroid the fibroblasts or the ECM are present. Here, using the new clearing protocol, we characterized the ECM location and production over time in our tumour spheroids, as a function of the fibroblast amount in the tissues. Finally, we performed specific immunostaining of targeted markers for cell proliferation, hypoxia, cell death and collagen I, in the intact and cleared tumour spheroids, followed by imaging using confocal microscopy. 


\section{MATERIAL AND METHODS}

\subsection{Cell Culture}

4T1 mouse breast cancer cells and 3T3 mouse fibroblasts were obtained from American Type Culture Collection (ATCC, Rockville,MD) and cultured in Roswell Park Memorial Institute (RPMI) 1640 medium and Dulbecco's Modified Eagle's Medium (DMEM), respectively, as explained in the previous chapters. Cells were expanded in $75 \mathrm{~cm}^{2}$ culture flasks (CELLSTAR® Filter Cap $75 \mathrm{~cm}^{2}$, GREINER BIO-ONE, Kremsmünster, Austria) in an incubator under standard humidified conditions $\left(37^{\circ} \mathrm{C}, 5 \% \mathrm{CO}_{2}\right)$, and medium was refreshed every 2 days, as described thereafter. To retrieve the cells from the flask, medium was removed and cells washed with sterile Phosphate Buffer Saline (PBS) 1X buffer (Lonza, Basel, Switzerland) to remove dead cells. $2 \mathrm{~mL}$ of $0.25 \% \mathrm{w} / \mathrm{v}$ trypsin-EDTA (Thermo Fisher Scientific, Waltham, MA, USA) was added for $5 \mathrm{~min}$ to detach the cells from the surface. The cells were re-suspended in $8 \mathrm{~mL}$ of fresh DMEM medium (to block the action of the trypsin) and collected in $10 \mathrm{~mL}$ tubes. After centrifugation at $300 \mathrm{~g}$ for $3 \mathrm{~min}$, medium was removed and $2 \mathrm{~mL}$ of fresh DMEM medium was added. Cells were counted and 2 million cells were seeded in a new culture flask. The remaining cells were kept for further experiments, as described in the following section.

\subsection{SPHEROID PREPARATION AND CHARACTERIZATION}

\subsubsection{Spheroid preparation}

Tumour spheroids were prepared as described previously (chapter 3) in a microwell array, with cylindrical wells $800 \mu \mathrm{m}$ in diameter and $500 \mu \mathrm{m}$ deep, initially with a flat bottom. The well array was soft-embossed in a 35-mm diameter Petri dish as previously described by Sridhar et al. using a PDMS mold [13]. Additionally, a second microwell design was considered; with a deeper structure composed of a cylinder of $800 \mu \mathrm{m}$ in diameter and $400 \mu \mathrm{m}$ in depth, completed with a hemisphere of $800 \mu \mathrm{m}$ in diameter at the bottom (Figure 4-1a). A negative of the master mold with a well array of $800 \mu \mathrm{m}$ in diameter and $800 \mu \mathrm{m}$ in depth was designed with SolidWorks (Dassault Systèmes, Vélizy-Villacoublay, France) (Figure 4-1b), and produced with a 3D printer (Hunter, FlashForge, Zhejiang, China), with a lateral and vertical resolution of $62.5 \mu \mathrm{m}$ and $50 \mu \mathrm{m}$, respectively, and using Industrial Blend resin (Fun- 
To-Do, Alkmaar, The Netherlands). Soft lithography was performed by pouring a mixture of silicone base and curing agent (SYLGARD 184, Dow Corning, Midland, USA), in a 10:1 weight ratio, onto the $3 \mathrm{D}$ printed device and degassed. After curing $\left(60^{\circ} \mathrm{C}, 3 \mathrm{~h}\right)$, the resulting polydimethylsiloxane (PDMS) elastomer was peeled off, and used as master mold (Figure 41c) for soft embossing as mentioned above.

a)

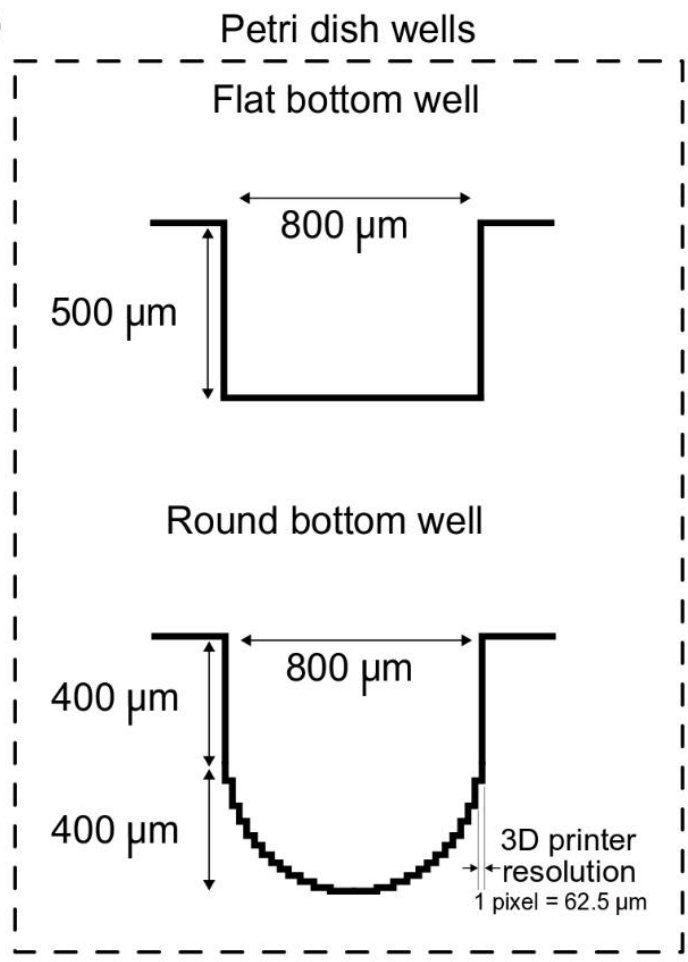

96 well-plate well

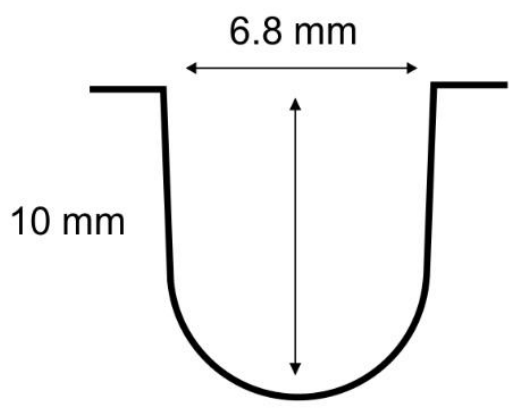

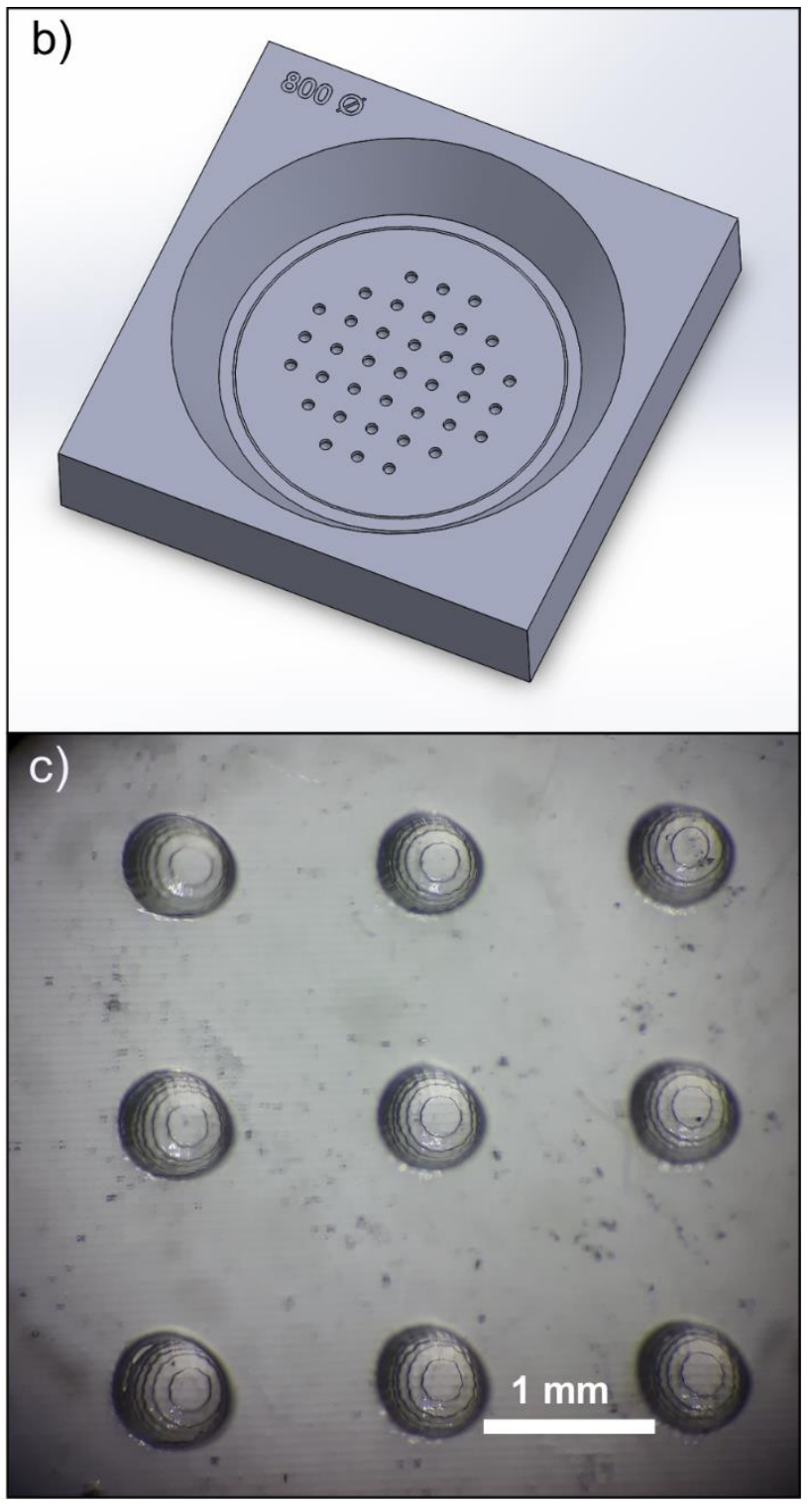

Figure 4-1 Platforms for spheroid production. a) Sketch of the different cylindrical well designs for spheroid preparation. Round-bottom well resolution in the Petri dish is limited by the $3 D$ printer pixel resolution $(62.5 \mu \mathrm{m})$ b) SolidWorks perspective view of the printed mould, negative of the microwell array (round bottom wells) master mould. c) Soft embossed well array inside a Petri dish. Each concentric ring inside the wells indicates a change in depth of the wells of $50 \mu \mathrm{m}$, due to the maximum resolution of the $3 D$ printer. 
Cells were seeded in the microwell array treated with a cell repellent coating, Pluronic F-127, as described in chapter 5. Spheroids were prepared using different cellular compositions, by varying the amount of fibroblasts in the tumour model. Namely, we prepared tissues with the following compositions: 4T1 cancer mono-culture spheroids, 5:1 and 1:1 3T3:4T1 co-culture spheroids, and 3T3 mono-culture spheroids. After seeding the cells in the devices, the spheroids were kept in culture for 3, 7, or 10 days, their medium being refreshed every 2 days. Spheroids were next collected in an Eppendorf tube, washed 3x with PBS, fixed in a $4 \%$ formaldehyde solution overnight at $4^{\circ} \mathrm{C}$, and washed again $3 \mathrm{x}$ in PBS.

As a control, spheroids were also prepared by seeding 30,000 cells in a 96-well plate with a round bottom and pre-treated with a cell-repellent coating (Corning, NY, USA), which a standard platform for cell aggregation. The cells were incubated for 3 or 7 days, prior to collection and fixation of the spheroids.

\subsubsection{Size and sphericity characterization}

To evaluate the size and sphericity of the spheroids, the tissues were embedded in a hydrogel cube, and imaged from different angles. To embed the spheroids, a 3\% w/w agarose solution was prepared, by dissolving agarose powder (Ultra-Pure Agarose, Thermo Fisher Scientific) in distilled water at $70^{\circ} \mathrm{C}$. Fixed spheroids were mixed with the agarose before its gelation, and poured in a 6-well plate (Corning). After complete agarose gelation (30 min at $20^{\circ} \mathrm{C}$ ), cubes of agarose containing a single spheroid each were cut. Images of the different faces of the cubes were taken (one in each dimension), using EVOS-FL microscope (Thermo Fisher Scientific), and the diameter and eccentricity of the spheroids in each dimension were measured using ImageJ software (NIH, Bethesda).

\subsection{TISSUE CLEARING}

\subsubsection{Sample preparation}

The tissue clearing protocol followed the one proposed by VISIKOL ${ }^{\mathrm{TM}}$ [14]. Unless indicated otherwise, all preparation steps were performed at room temperature. Firstly, antigen retrieval was performed by placing the spheroids in dry methanol for $2 \mathrm{~min}$, followed by $20 \%$ v/v DMSO in methanol for $2 \mathrm{~min}$, and dry methanol again for $2 \mathrm{~min}$. Finally, the samples were washed with $1 \% \mathrm{w} / \mathrm{v}$ Triton ${ }^{\mathrm{TM}} \mathrm{X}-100$ in PBS for $2 \mathrm{~min}$. The spheroids were next incubated, with slow agitation, in a "penetration buffer" (PBS with $0.2 \% \mathrm{w} / \mathrm{v}$ Triton $^{\mathrm{TM}} \mathrm{X}-100,0.3 \mathrm{M}$ glycine and 20\% w/v DMSO) for $30 \mathrm{~min}$, followed by a "blocking buffer" (PBS with 6\% w/v 
Bovine Serum Albumin (BSA), 0.2\% w/v Triton ${ }^{\mathrm{TM}} \mathrm{X}-100$ and 10\% v/v DMSO ) for $30 \mathrm{~min}$ at $37^{\circ} \mathrm{C}$

\subsubsection{Antibody staining}

The blocking buffer was removed and a primary antibody suspended in an "antibody buffer" (PBS with 0.2\% w/v Tween ${ }^{\text {TM }} 20,100 \mu \mathrm{g} / \mathrm{mL}$ heparin, 3\% w/v donkey serum, and 5\% v/v DMSO) was added to the samples and let for incubation for $3 \mathrm{~h}$. The spheroids were washed with a "Washing Buffer" (10X PBS with 2\% Tween TM 20 and $100 \mu \mathrm{g} / \mathrm{mL}$ heparin) $3 \mathrm{X}$ for 5 min each. The same procedure was repeated for the secondary antibody. Finally, the spheroids were washed $2 \mathrm{X}$ in dry methanol and imaged, or stored at $5^{\circ} \mathrm{C}$.

To investigate the specificity of the staining (see chapter 3) on the cleared spheroids, phalloidin (Actin Green, \#R37110, Thermo Fisher Scientific) was first tested, followed by an antibody staining with E-cadherin/CDH1 polyclonal primary antibody (\#PA5-32178, Thermofisher).

To evaluate the spatial layer-by-layer "structure" of the spheroids, different markers related to cell survival and proliferation were tested: Ki-67 (antibody \#ab15580, abcam) to stain the proliferating cells, Hypoxia Induced Factor 1-alpha (HIF-1 $\alpha$ antibody, \#PA1-16601, Thermo Fisher Scientific) to stain hypoxic cells, and anti-cleaved Caspase3 (antibody \#ab2302, abcam) to stain apoptotic cells.

Finally, to study the influence of the stromal cells, fibroblasts here, on the production of Extracellular Matrix (ECM), collagen I was stained using collagen I antibody (\#1310-01, SouthernBiotech, Birmingham, AL, USA).

Each primary antibody was labelled with a green fluorescent secondary antibody goat antirabbit secondary antibody, Alexa Fluor 488 (\#A-11034, Thermo Fisher Scientific) or donkey anti-goat secondary antibody, Alexa Fluor 488 (\#A-11055, Thermo Fisher Scientific). All spheroids were labelled with a nuclear counterstaining (Hoechst 33342, NucBlue, \#R37605, Thermo Fisher Scientific), added together with the secondary antibody.

\subsubsection{Transparency characterization}

Images of the tissues were taken at different steps in the clearing protocol. Transparency of the tissues was measured using the intensity signal (using ImageJ) of the image background and dividing it by the average of the intensity signal inside the spheroid. Results were presented as a percentage of transparency, using the following equation: 


$$
\text { transparency }(\%)=\frac{\text { intensity image }}{\text { intensity background }} * 100
$$

\subsection{IMAGING}

High resolution imaging was performed using confocal microscopy (Nikon A1R-A1, Tokyo, Japan) with a 20x objective. Spheroids treated with iDISCO and in dry methanol were placed on a glass coverslip with a PDMS O-ring ( $5 \mathrm{~mm}$ inner diameter). The methanol was let to evaporate, and the spheroids were immersed in VISIKOL HISTO-M mounting solution. Images were acquired with a sample thickness of $c a .20 \mu \mathrm{m}$.

Images were acquired at the bottom and mid-height of each spheroid, which allowed evaluating whether tissues were completely cleared. Finally, when useful, 3D reconstruction was performed by acquiring multiple images (z-stack) of the bottom half hemisphere of the spheroid and using the 3D tool of the confocal microscope user software (NIS, Nikon).

\section{ReSults \& Discussion}

\subsection{SPHEROID FORMATION}

As already mentioned, tumours are characterized by the presence of gradients of oxygen and nutrients. According to the literature, to present a necrotic core, a tumour spheroid must be at least $400 \mu \mathrm{m}$ in diameter $[11,15]$. The spheroids we produced in this thesis are ranging from $c a .200 \mu \mathrm{m}$ to $c a .500 \mu \mathrm{m}$. Gradients inside the spheroids must however be isotropic. Therefore, to include a necrotic core, the spheroid must be at least $400 \mu \mathrm{m}$ in dimension in all directions. We examined and compared three different methods of spheroid production, and characterized the shape of the resulting spheroids in 3D. First, we imaged each spheroid from the top (standard imaging technique, averaged from 12 spheroids per conditions), and measured its shortest dimension (or 2x smallest radius), which corresponded to the minimum distance of the gradient in the tissue. We next imaged the spheroids from different angles (following the cartesian coordinates) after their encapsulation in agarose cubes, and evaluated their eccentricity (averaged from 4 spheroids per condition, considering the projection of the spheroid as an ellipse), expressed as:

$$
e=\sqrt{1-\frac{b^{2}}{a^{2}}}
$$


with $a$ the major axis of the ellipse, and $b$ the minor axis, orthogonal to the first diameter (in a perfect circle $\mathrm{a}=\mathrm{b}$, so $\mathrm{e}=0$ ).

First, the spheroids were prepared in an array of microwells of $800 \mu \mathrm{m}$ in diameter and $500 \mu \mathrm{m}$ in depth, as presented in chapter 2 . When observed from the top (Z-axis), the spheroids appeared as circular (Figure 4-2.a). Their average minimum diameter was $298 \pm 47 \mu \mathrm{m}$ for (4T1) mono-culture spheroids, with a low eccentricity of $c a .0 .35 \pm 0.05$. Co-culture spheroids in a 5:1 3T3:4T1 ratio presented a minimum diameter of $514 \pm 64 \mu \mathrm{m}$ with an eccentricity of ca. $0.47 \pm 0.12$. Also, and as seen in chapter 3 , after 3 days of incubation, the 4T1 mono-culture spheroids were shedding increasing amounts of cells, losing thereby their structural integrity. However, when observing them along 3 orthogonal axes (Figure 4-2.a), these spheroids appeared relatively flat, with an average eccentricity of $0.73 \pm 0.06$ ( $\mathrm{X}$ and $\mathrm{Y}$ axes) for the 4T1 mono-culture spheroid, and $0.76 \pm 0.08$ for the 5:1 co-culture (Figure 4-2.c).

Next, we decided to change the shape of the microwells to use microwells with a round bottom. The resulting spheroids had a minimum diameter of $334 \pm 15 \mu \mathrm{m}$ for the 4T1 monoculture spheroids, and a minimum diameter of $482 \pm 42 \mu \mathrm{m}$ for the 5:1 3T3:4T1 co-culture spheroids (Figure Appendix I). Looking at the spheroids 3D projections along 3 orthogonal axes revealed more spherical structure with an eccentricity of $0.44 \pm 0.07$ (in the $\mathrm{X}$ and $\mathrm{Y}$ axes) for the 4T1 mono-culture spheroid and $0.49 \pm 0.13$ for the 5:1 co-culture MCTS (Figure 4-2.b).

Additionally, spheroids were prepared in a conventional 96-well plate with wells having a round bottom (maximum diameter $6.8 \mathrm{~mm}, 10 \mathrm{~mm}$ in height, Figure 4-1.a). The resulting spheroids presented a much larger minimum diameter: $673 \pm 16 \mu \mathrm{m}$ for 4T1 monoculture, and $752 \pm 31 \mu \mathrm{m}$ for 5:1 co-culture spheroids (Figure 4-S2). It was also noted that, at this dimension, the mono-culture spheroids, presented after 4 days a rather fragile structure, and would break when manipulated. Considering the different projections demonstrated that the spheroids were extremely flat (eccentricity $c a$. 0.9). Based on these results, we decided to solely prepare spheroids in arrays of round bottom microwells. 
a)
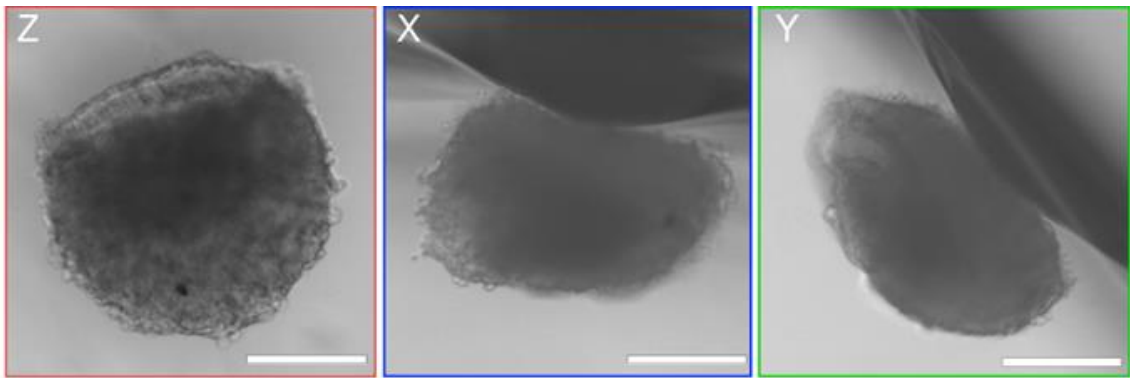

b)
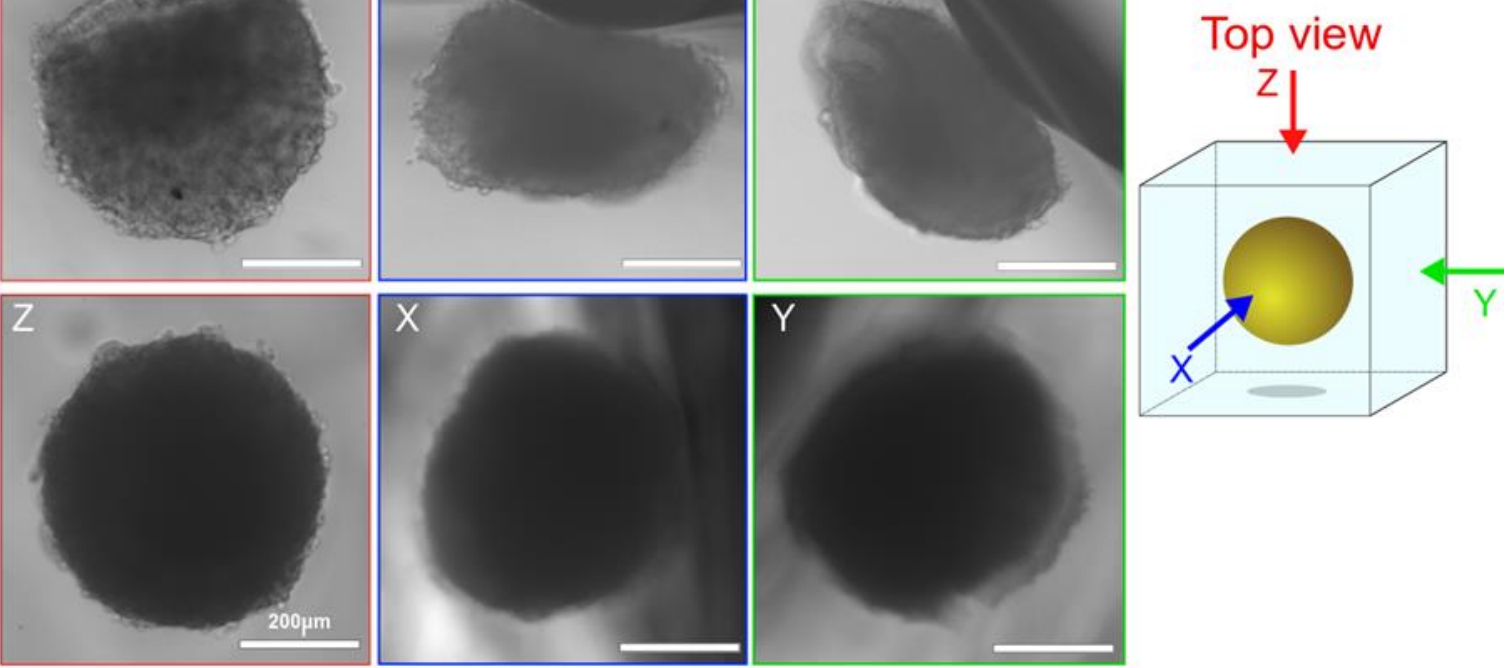

c)

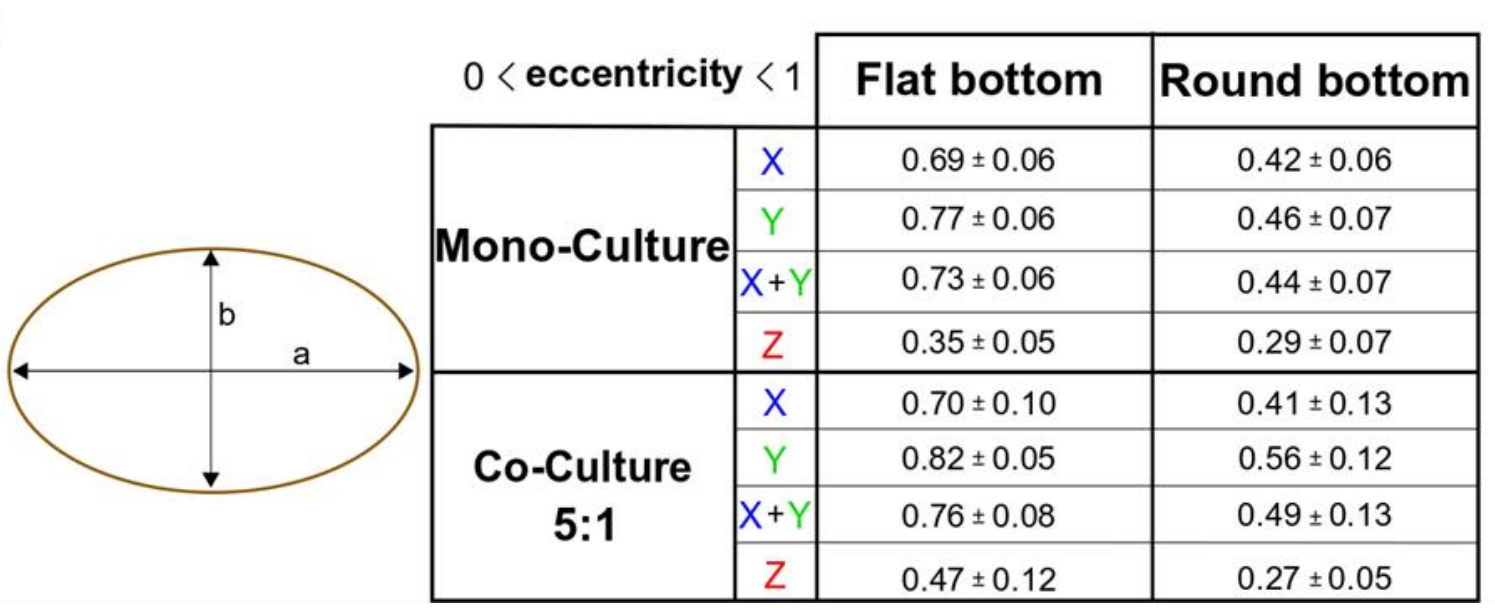

Figure 4-2 Shape characterization of spheroids. a,b) Projections of a $4 T 1$ mono-culture spheroid formed in a flat (a) or a round (b) bottom microwell, and encapsulated in an agarose cube, from different cartesian angles. c) Average eccentricity of the projections of $4 T 1$ mono-culture spheroids and 5:1 3T3:4T1 co-culture spheroids, formed in flat or round bottom microwells, along the three cartesian axes. Number of spheroids per conditions $n=4$.

\subsection{TISSUE CLEARING}

\subsection{1 iDISCO principle}

The iDISCO protocol was initially established for large ex vivo tissues (> $1 \mathrm{~mm}$ in thickness), and adapted by VISIKOL ${ }^{\mathrm{TM}}$ to investigate smaller tissues like spheroids. In the initial protocol, after sample fixation in formaldehyde, methanol and hydrogen peroxide were 
used to bleach the tissues, to reduce autofluorescence, and improve antibody diffusion. Next, combination of glycine and heparin was used to help reduce immunolabeling background. Finally, the tissues were exposed to a cycle of non-ionic detergents (i.e., Tween-20 and Triton$\mathrm{X}$ ) and organic solvent (i.e., methanol), dehydrating and rehydrating the samples, while removing the cell membrane lipids [5].

Here, due to the relatively small size and fragile structure of the spheroids, only methanol was used to bleach the tissues, followed by incubation in heparin and glycine, to reduce autofluorescence. A single cycle of dehydration (methanol evaporation) and rehydration (in VISIKOL HISTO-M) was sufficient to completely clear the tissues (Figure 4-3.c). Noteworthy, the spheroids were found to shrink as a result of the dehydration, and did not return to their original size after rehydration, a phenomenon also reported by the developers of the iDISCO protocol [5].
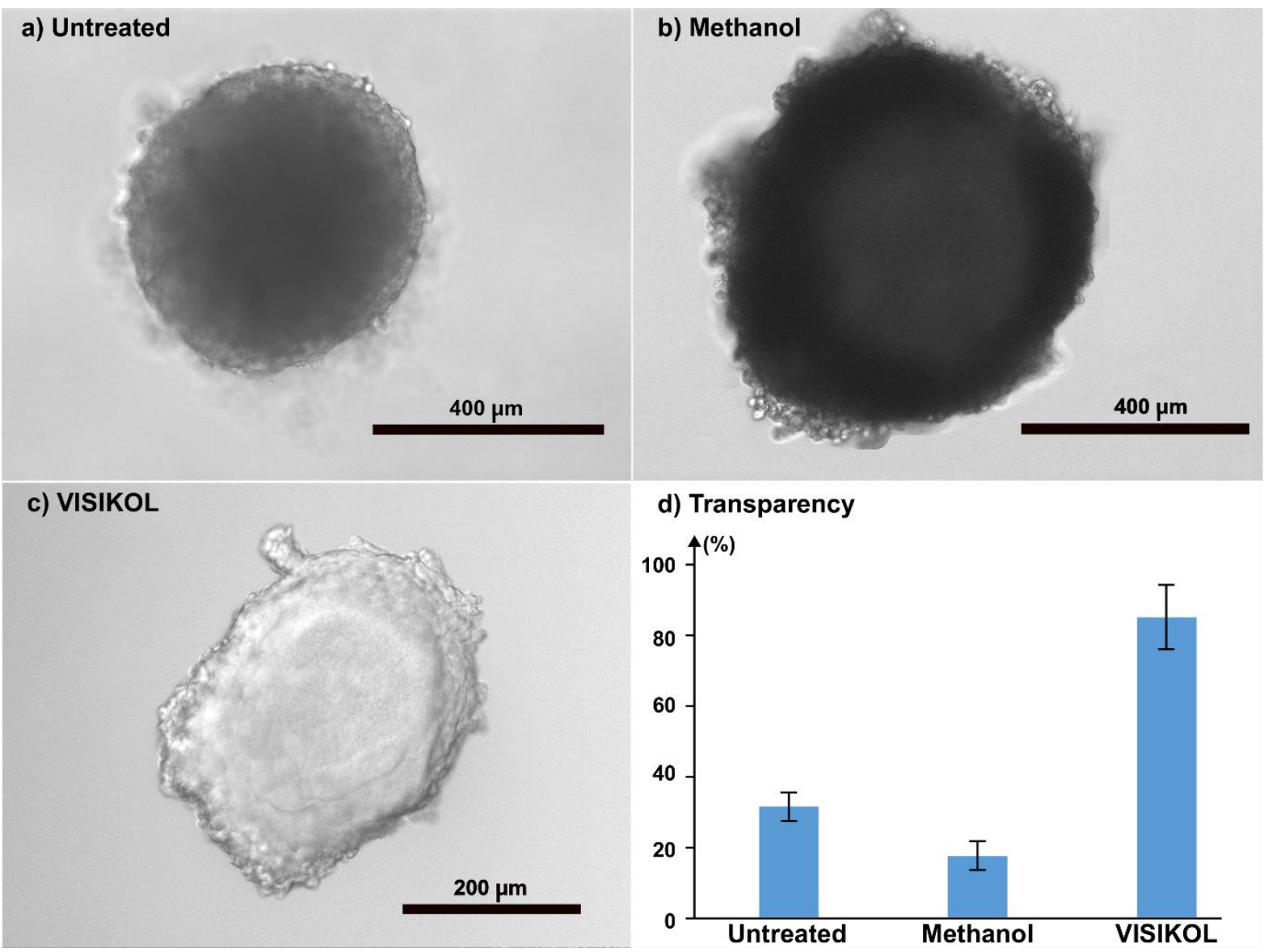

Figure 4-3 Images of 5:1 3T3:4T1 co-culture spheroids fixed in formaldehyde, (a) untreated, (b) in methanol, and (c) in VISIKOL HISTO-M solution. d) Average transparency of the spheroids in the different aforementioned solutions. Spheroids imaged per condition $n=6$. 


\subsubsection{Transparency characterization}

After preparation of the tissues following the VISIKOL approach, their transparency was quantified and compared for cleared and uncleared tissues (Figure 4-3). Prior to placing the spheroids in the VISIKOL refractive index matching solution HISTO-M, the spheroids (in methanol) were still quite opaque (ca. 22\%) (Figure 4-3.b). When the methanol was entirely evaporated, the spheroids in HISTO-M presented a very high level of transparency ( $c a .85 \%$ ) (Figures 3.c,d). Importantly, if the VISIKOL HISTO-M solution was added before complete evaporation of the methanol, the hydrophobic nature of the mounting solution led to the encapsulation of methanol in the tissue, which significantly affected the transparency of the final tissue.

Additionally, we noticed that the spheroids tended to adhere to the substrate during methanol evaporation, leading to a small collapse of the spheroids (Figure Appendix 3.a). By activating the glass coverslip with a plasma treatment, the surface became temporarily highly hydrophilic and spheroids no longer adhered to the substrate.

\subsection{IMMUNOSTAINING}

\subsubsection{Immunostaining specificity}

To first assess whether the non-specific binding issue as discussed in chapter 3 was resolved, cleared tumour spheroids were stained with phalloidin anti F-actin (Figure 4-4.a) and anti E-cadherin antibody (Figure 4-4.b). Firstly, both small (phalloidin $0.79 \mathrm{kDa}$ ) and large molecules (E-cadherin $97 \mathrm{kDa}$ ) successfully penetrated down to the core of the tumour spheroids, giving rise to a homogeneous distribution of the fluorescence intensity, suggesting that the penetration of antibodies was not limited.

Secondly and most importantly, the staining appeared to be specific. Indeed, the fluorescent signal attached to the E-cadherin antibody was limited to the cell membrane, suggesting that E-cadherin protein was not (partially) denaturated as a result of the clearing process (Figure 4-S4).

Finally, the only limitations noticed using this protocol were the occasional incomplete clearing of the spheroid core (Figure 4-S3.b), and the associated restricted imaging depth. The first issue was most likely due to incomplete replacement of the washing buffer by the methanol, leading to an imperfect drying of the tissue by evaporation, prior to adding the VISIKOL HISTO-M solution. By increasing the evaporation time, the recurrence of the effect 
was greatly reduced. The second limitation, also mentioned in chapter 2 with the CLARITY protocol, was the decrease in sharpness of the image due to residual light diffraction, and the limited focal distance of the confocal microscope objectives (20x objective focal length $=1$ $\mathrm{mm})$.

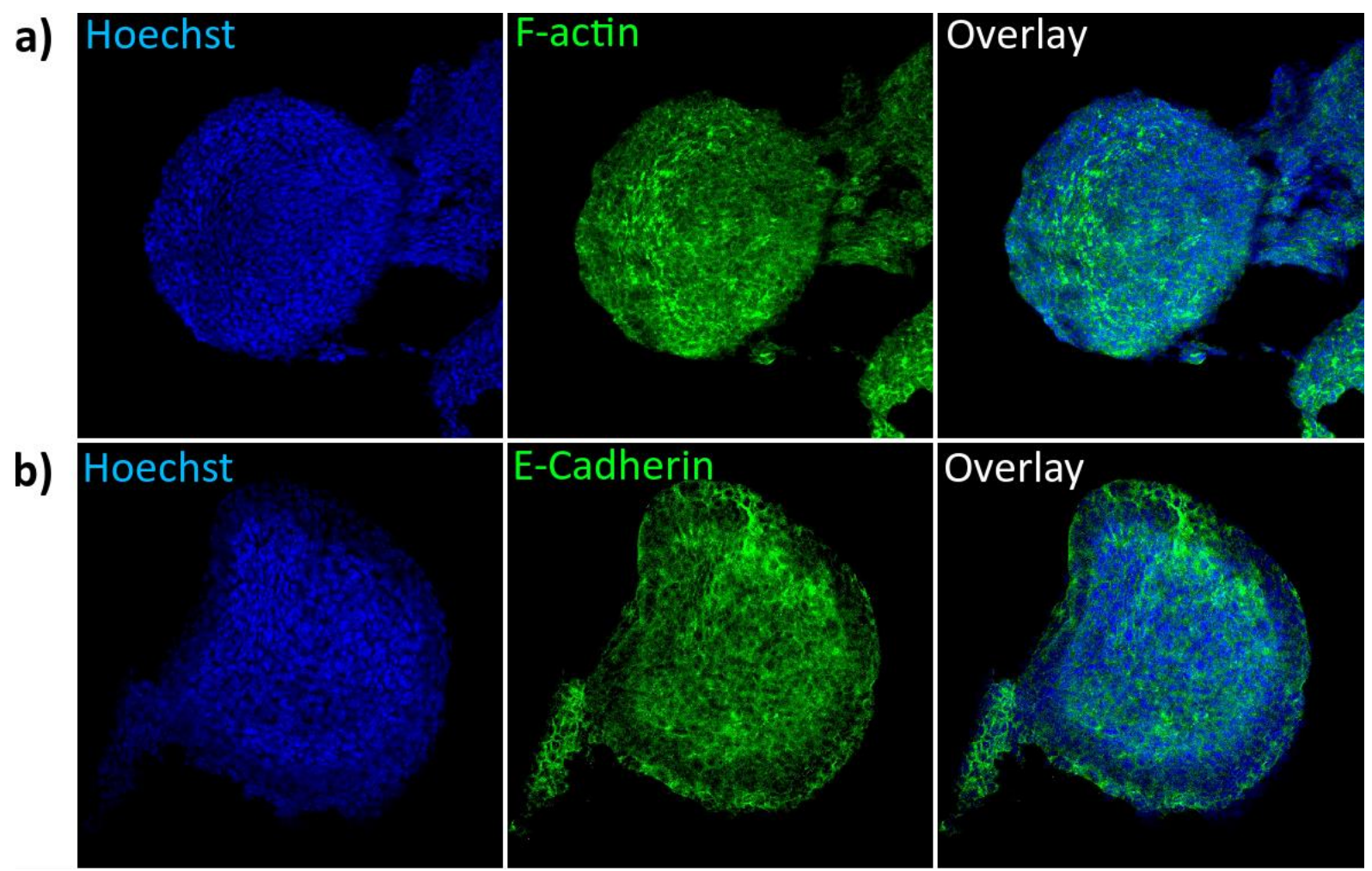

Figure 4-4 Characterization of immunostaining. 3T3:4T1 5:1 co-culture spheroids cleared after 7 days of culture, treated with VISIKOL, and stained with Hoechst (blue) and (a) Phalloidin or (b) anti ECadherin antibody (green).

\subsubsection{Oxygen and nutrient gradients}

\subsubsection{Proliferation}

Immunostaining was performed on VISIKOL cleared tissues, targeting different cell phenotypes (i.e., proliferative, hypoxic and apoptotic behavior). For the proliferative zone, Ki67, a protein only expressed in proliferating cells during all active phases of the cell cycle, was considered (Figure 4-5). Spheroids were cultured for 3, 7 or 10 days, prior to clearing and immunostaining. At day 3, almost all cells in the spheroids exhibited a proliferative behavior, with cells positive to anti $\mathrm{Ki}-67$ antibody being found up to $102 \pm 7 \mu \mathrm{m}$ from the edge of 4T1 mono-culture spheroids (ca. $149 \mu \mathrm{m}$ in radius) (Figure 4-5.a). At day 7 almost no staining was 
found inside the spheroid, and cells positive to anti Ki-67 antibody only formed a shell with a thickness of $22 \pm 6 \mu \mathrm{m}$ (Figure 4-5.b). At day 10, Ki-67 was only observed in some cells at the spheroid surface, and the number of cells expressing Ki-67 had overall greatly decreased. The decrease in signal on the outer rim of the spheroids over time suggested that the cells on the outer layer eventually also stopped proliferating, explaining why during incubation the spheroids did not increase in size over time.

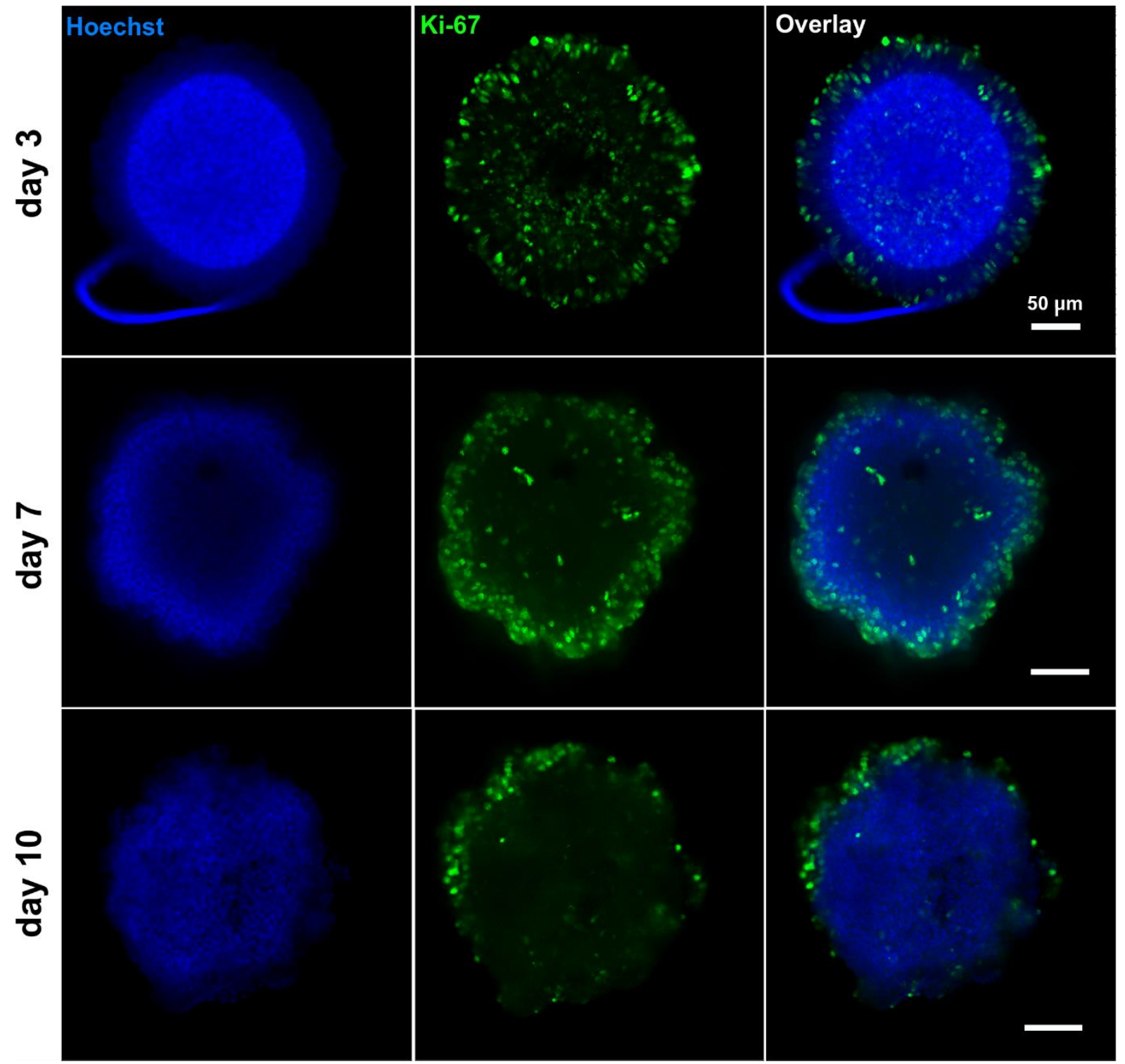

Figure 4-5 Mono-culture 4T1 spheroids treated with VISIKOL and stained with Hoechst (blue) and anti Ki-67 antibody (green), after 3,7, and 10 days of incubation in the microwell array. Cells expressing Ki-67 gradually localize toward the spheroid outer rim over time. 


\subsubsection{Hypoxia}

A highly investigated marker for hypoxia is Hypoxia Induced Factor $1 \alpha$ (HIF-1 $\alpha$ ), a protein responsible for the transcriptional activation of many genes (e.g., VEGF), and whose degradation is regulated by $\mathrm{O}_{2}$-dependent prolyl hydroxylation $[16,17]$. As a result, HIF-1 $\alpha$ is overexpressed in hypoxic tissues, such as tumours. This protein, was found overexpressed throughout the tissue after already 3 days (Figure 4-6). Indeed, HIF-1 $\alpha$ is secreted by the cells, and can as such diffuse throughout the tissues. Therefore here, HIF-1 $\alpha$ staining was insufficient to isolate the hypoxic regions in the spheroid, but could still confirm their existence.

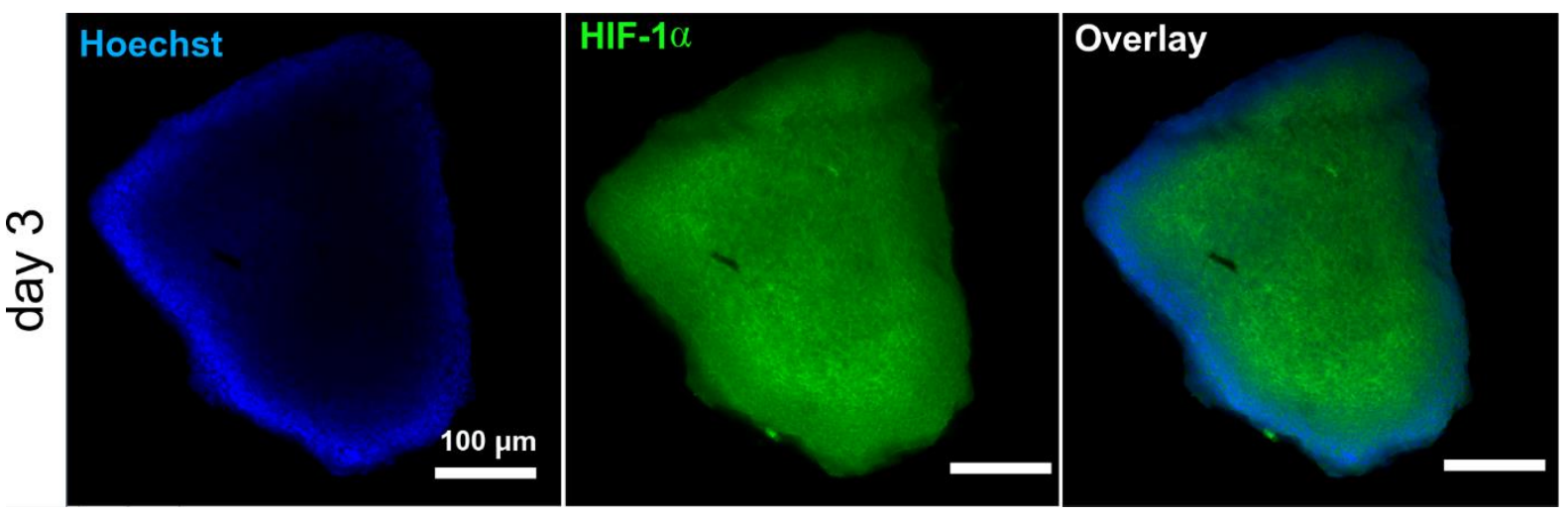

Figure 4-6 3T3:4T1 5:1 co-culture spheroid treated with VISIKOL and stained with Hoechst (blue) and HIF-1 $\alpha$ antibody (green).

\subsubsection{Apoptosis}

Cysteine-ASPartic Acid proteaSE 3, or caspase-3, is a protein secreted as a zymogen (inactive protein) and, like other members of the caspase family, is cleaved, and activated in apoptotic cells [18]. As previously mentioned, the process that turns a cell into a cancer cells is accompanied with a down-regulation of apoptosis [19]. In healthy tissues, apoptosis can be triggered by multiple processes, for instance as a response to hypoxia, and activated by HIF$1 \alpha$ [20]. We have previously showed that HIF-1 $\alpha$ was expressed in our spheroids, and so here, we investigated whether the increased amount of HIF-1 $\alpha$ resulted in apoptosis.

In our experiments, we noticed a rather homogeneous yet low distribution of apoptotic cells within the tissues (Figure 4-7). Moreover, the amount of cells expressing caspase-3 did not significantly vary overtime, suggesting that those cells were constantly being either expelled from the spheroids, or consumed by neighboring cells through a process called autophagy [21]. 


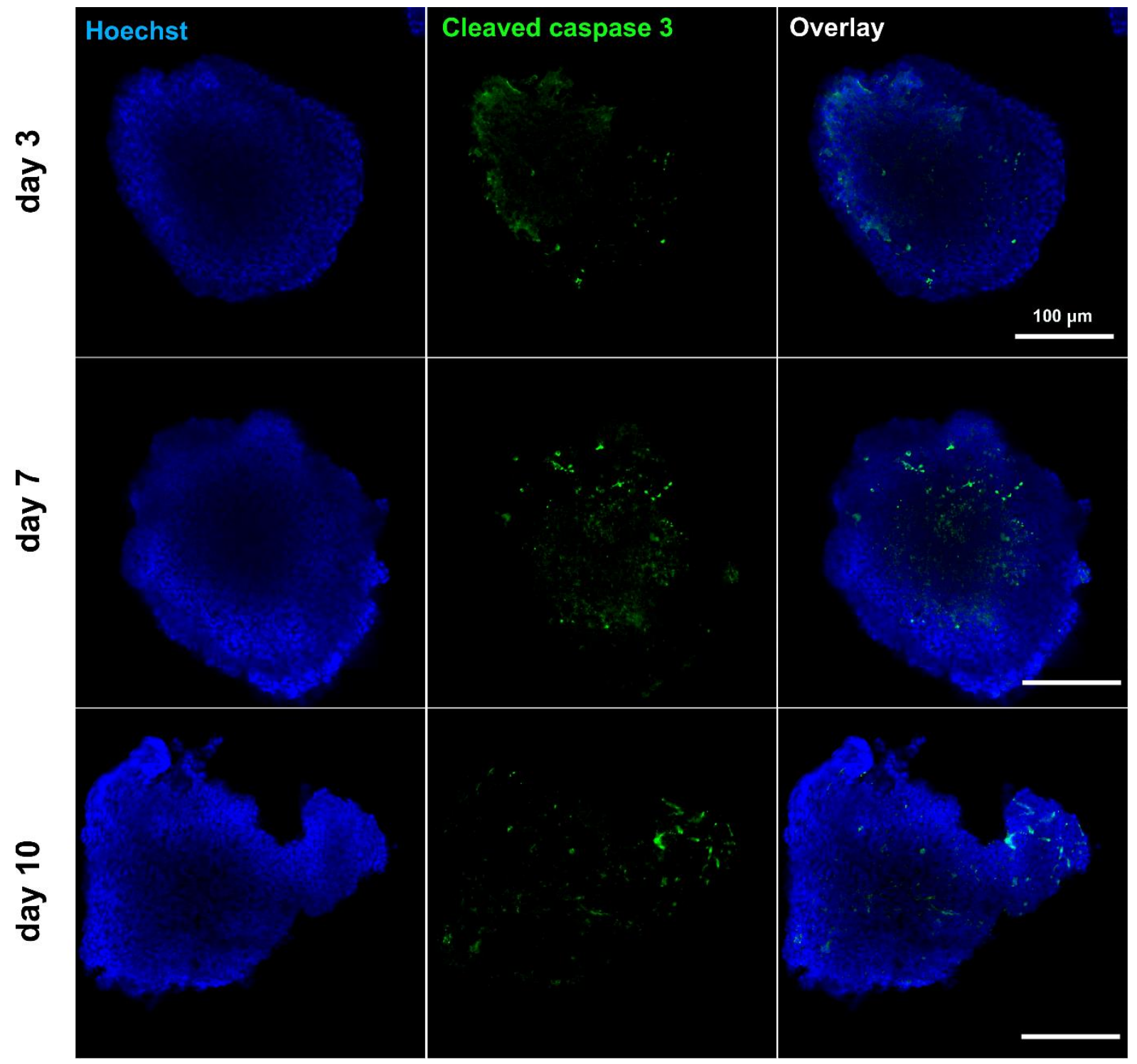

Figure 4-7 3T3:4T1 1:1 co-culture spheroids treated with VISIKOL and stained with Hoechst (blue) and anti-cleaved caspase 3 antibody after 3, 7 or 10 days of incubation in the microwell array.

\subsubsection{ECM production}

As mentioned previously in this thesis, fibroblasts and other stromal cells are responsible, among other functions, for the production of extracellular matrix (ECM). We stained the spheroids with anti-collagen I antibody, as collagen I is a representative element of the ECM. Spheroids were cultured as before for 3, 7 or 10 days, prior to clearing and immunostaining. At day 3 in the 5:1 3T3:4T1 co-culture spheroids, collagen I was mostly found 


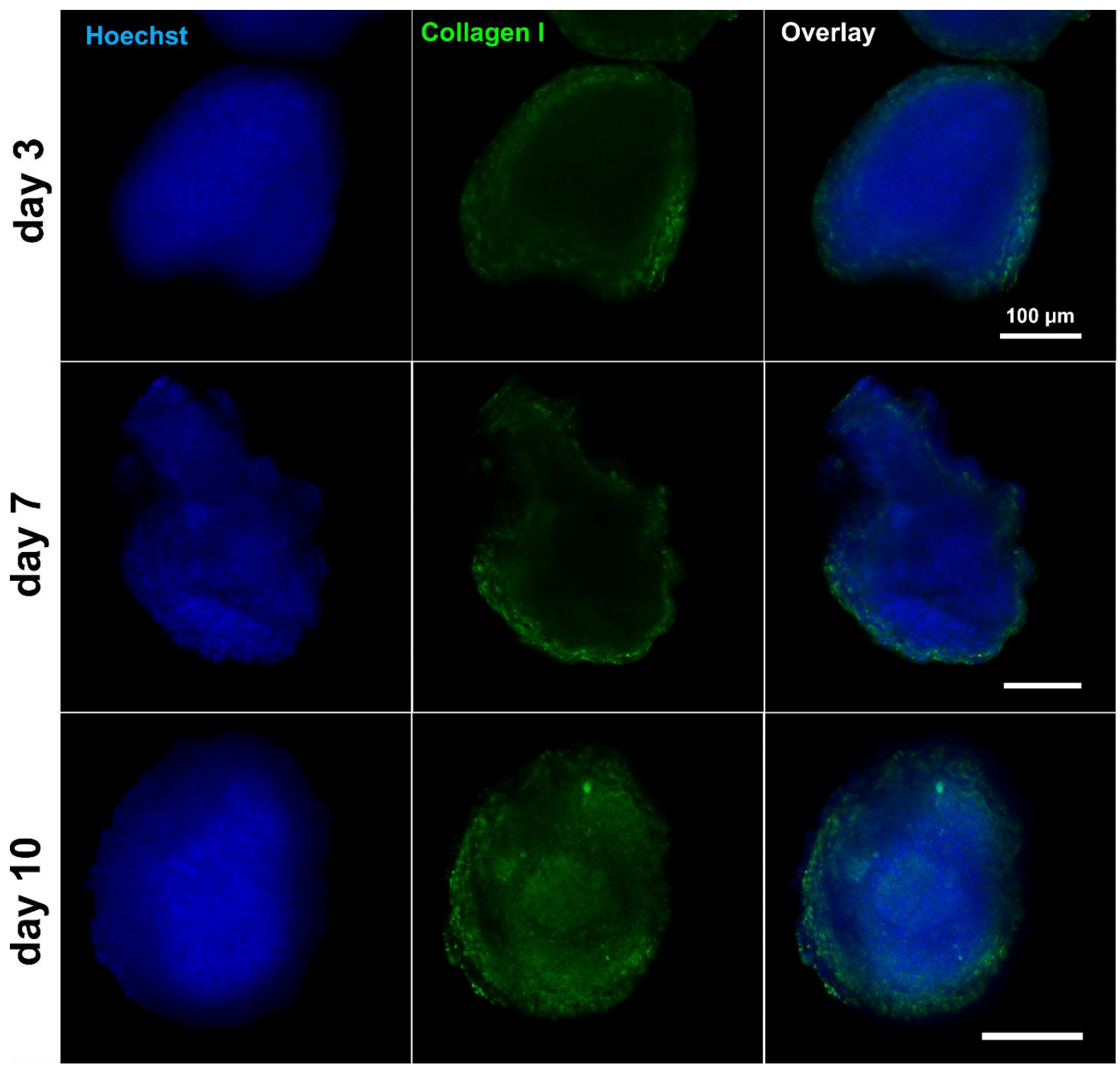

Figure 4-8 3T3:4T1 5:1 co-culture spheroid, treated with VISIKOL and stained with Hoechst (blue) and Collagen I antibody (green) after 3,7 and 10 days of incubation.

at the periphery of the tissues (Figure 4-8 and 4-S6.a). Similar results were observed for the 1:1 co-culture spheroids (data not shown). Over time, the amount of collagen I, and supposedly by extension the whole ECM increased and filled the entire spheroid by day 10 (Figure 10 and Figure appendix 6.b). In the 3T3 mono-culture spheroids however, collagen was found throughout the entire spheroid already after 3 days, indirectly suggesting that the fibroblasts present in the co-culture spheroids, were essentially located in the outer layer of the spheroids, a result which is in good agreement with previous studies [22]. 


\section{CONClusion}

By improving our microwell array design, replacing the flat bottom wells with round bottom ones, we produced here spherical tissues reaching at least $400 \mu \mathrm{m}$ in diameter, and potentially presenting isotropic gradients of oxygen and nutrients.

Using the iDISCO protocol followed by the VISIKOL HISTO-M mounting solution, we completely cleared the tumour spheroids. The resulting tissues shrunk as a result of the lipid removal, but the protein $3 \mathrm{D}$ configuration was not altered as was the case with the CLARITY protocol. On the cleared spheroids, we successfully performed immunostaining, targeting reference proteins (i.e., F-Actin and E-Cadherin), with high specificity regarding their affinity.

Looking at markers related to the different hypoxia levels, we were able to identify a change in the proliferative layer over time: at first englobing the entire spheroids, the proliferative layer gradually reduced to become limited to the outer rim, until eventually only including the first layer of cells. We were also able to confirm the presence of hypoxia inside the tumour spheroid by looking at the expression of HIF-1 $\alpha$. Also, by looking at apoptotic markers, we concurred with the knowledge that, as a result of genetic mutation, the cancer cells were unable to undergo the process of apoptosis. Finally, looking the extracellular matrix (ECM) production, we were able to observe the increase in collagen I production, and how the presence of fibroblasts influenced it.

The results presented here demonstrate the validity of the clearing method as an in situ imaging technique of 3D tissue, and could be used to ascertain the location of specific proteins for the development of targeted therapeutics. 


\section{REFERENCES}

1. Chung, K., et al., Structural and molecular interrogation of intact biological systems. Nature, 2013. 497(7449): p. 332-7.

2. Lee, E., et al., ACT-PRESTO: Rapid and consistent tissue clearing and labeling method for 3-dimensional (3D) imaging. Scientific Reports, 2016. 6.

3. Azaripour, A., et al., A survey of clearing techniques for 3D imaging of tissues with special reference to connective tissue. Prog Histochem Cytochem, 2016. 51(2): p. 923.

4. Becker, K., et al., Chemical Clearing and Dehydration of GFP Expressing Mouse Brains. Plos One, 2012. 7(3).

5. Renier, N., et al., iDISCO: A Simple, RapidMethod to Immunolabel Large Tissue Samples for Volume Imaging. Cell, 2014. 159(4): p. 896-910.

6. Hama, H., et al., Scale: a chemical approach for fluorescence imaging and reconstruction of transparent mouse brain. Nature Neuroscience, 2011. 14(11): p. 1481-U166.

7. Ke, M.T., S. Fujimoto, and T. Imai, SeeDB: a simple and morphology-preserving optical clearing agent for neuronal circuit reconstruction. Nat Neurosci, 2013. 16(8): p. 1154-61.

8. Villani, T.S., A.R. Koroch, and J.E. Simon, An Improved Clearing and Mounting Solution to Replace Chloral Hydrate in Microscopic Applications. Applications in Plant Sciences, 2013. 1(5).

9. Ariel, P., A beginner's guide to tissue clearing. International Journal of Biochemistry \& Cell Biology, 2017. 84: p. 35-39.

10. Barisam, M., et al., Prediction of Necrotic Core and Hypoxic Zone of Multicellular Spheroids in a Microbioreactor with a U-Shaped Barrier. Micromachines (Basel), 2018. 9(3).

11. Riffle, S., et al., Linking hypoxia, DNA damage and proliferation in multicellular tumor spheroids. BMC Cancer, 2017. 17(1): p. 338.

12. Priwitaningrum, D.L., et al., Tumor stroma-containing 3D spheroid arrays: A tool to study nanoparticle penetration. J Control Release, 2016. 244(Pt B): p. 257-268.

13. Sridhar, A., et al., Microstamped Petri dishes for scanning electrochemical microscopy analysis of arrays of microtissues. PLoS One, 2014. 9(4): p. e93618.

14. VISIKOL. HISTO-M protocol. October 1, 2019]; Available from: https://visikol.com/products/visikol-histo-m/visikol-histo-m-getting-started-guide/.

15. Takagi, A., et al., Three-dimensional cellular spheroid formation provides human prostate tumor cells with tissue-like features. Anticancer Res, 2007. 27(1A): p. 45-53.

16. Lee, J.W., et al., Hypoxia-inducible factor (HIF-1)alpha: its protein stability and biological functions. Exp Mol Med, 2004. 36(1): p. 1-12.

17. Semenza, G.L., Targeting HIF-1 for cancer therapy. Nature Reviews Cancer, 2003. 3(10): p. 721-732.

18. Porter, A.G. and R.U. Janicke, Emerging roles of caspase-3 in apoptosis. Cell Death Differ, 1999. 6(2): p. 99-104.

19. Wong, R.S., Apoptosis in cancer: from pathogenesis to treatment. J Exp Clin Cancer Res, 2011. 30: p. 87.

20. Greijer, A.E. and E. van der Wall, The role of hypoxia inducible factor 1 (HIF-1) in hypoxia induced apoptosis. J Clin Pathol, 2004. 57(10): p. 1009-14.

21. Yun, C.W. and S.H. Lee, The Roles of Autophagy in Cancer. International Journal of Molecular Sciences, 2018. 19(11). 
22. Alkasalias, T., et al., Fibroblasts in the Tumor Microenvironment: Shield or Spear? Int J Mol Sci, 2018. 19(5).

\section{APPENDIX}

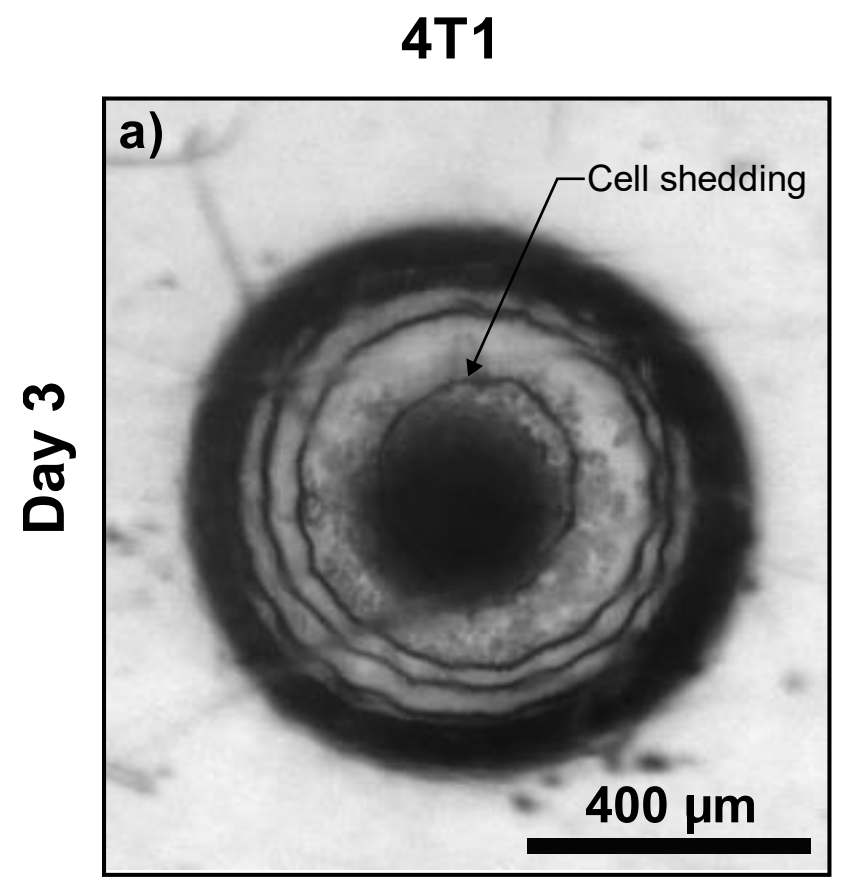

3T3:4T1 5:1

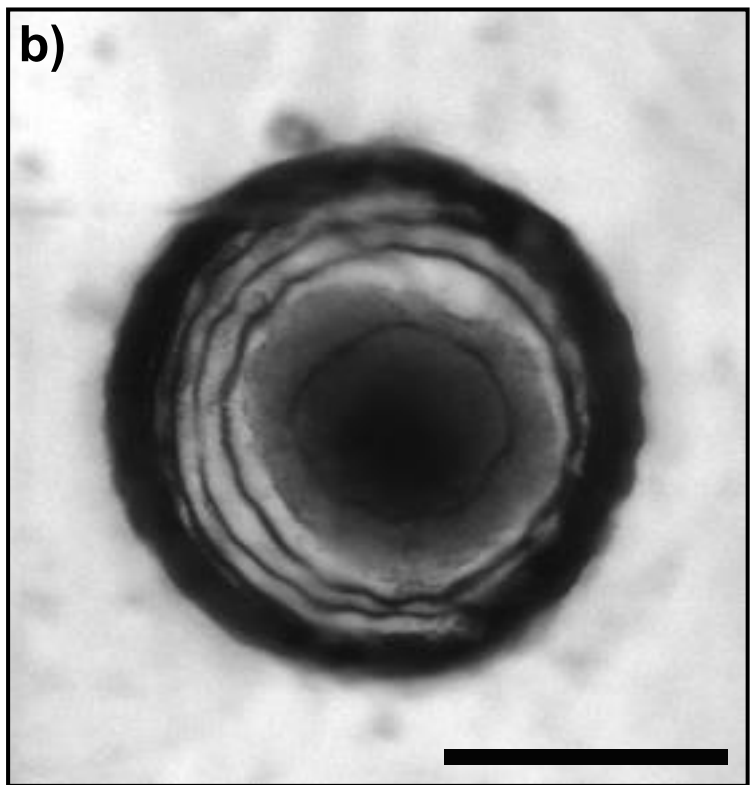

Figure 4-S1 (a) 4T1 mono-culture and (b) 3T3:4T1 5:1 co-culture spheroids aggregated in microwells with round bottom, after 3 days of incubation. Cells in the $4 T 1$ mono-culture spheroid shed from the tissue, preventing formation of a large spheroid. Number of spheroids for averaging $n=8$. 


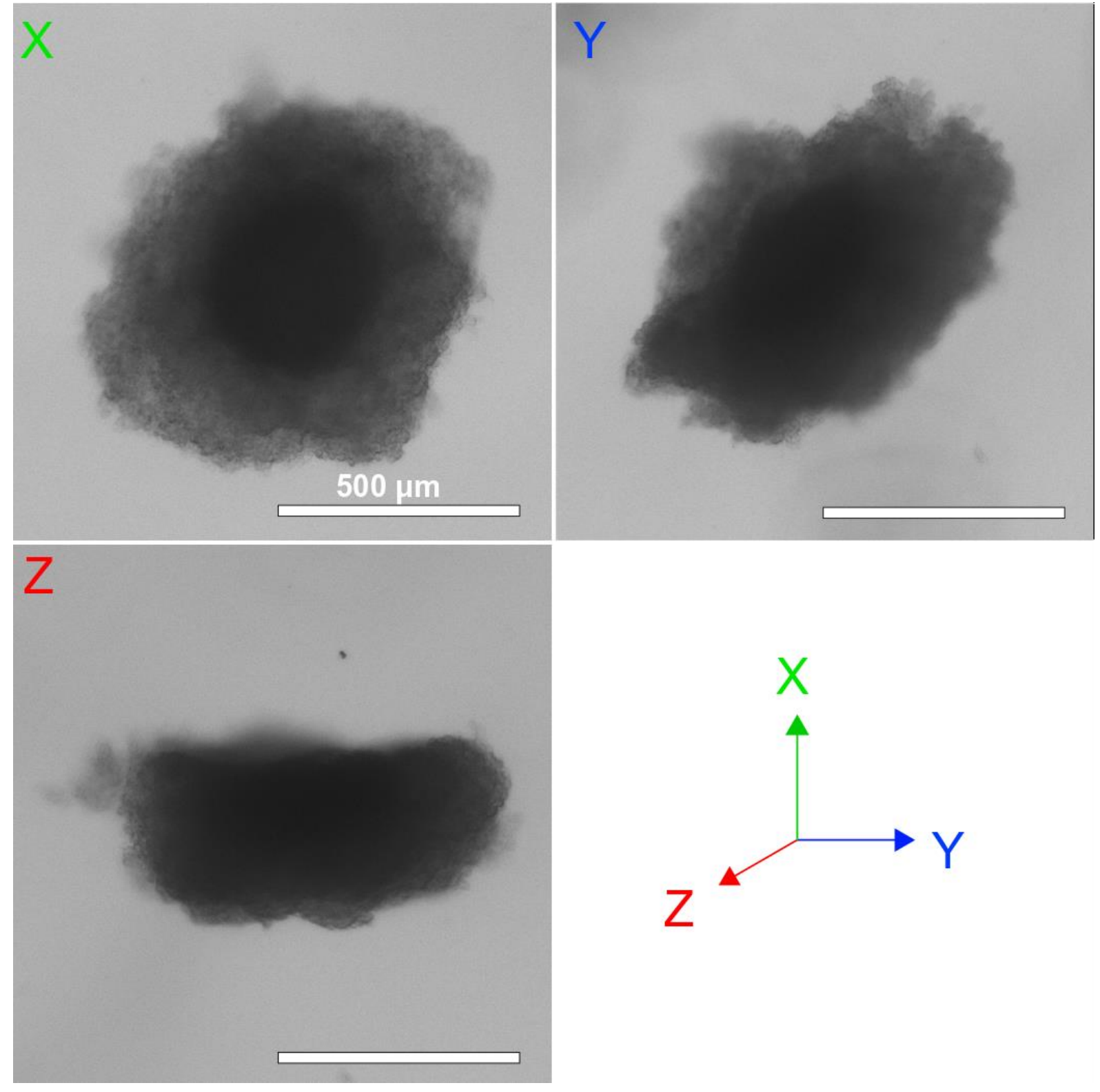

Figure 4-S2 3T3:4T1 5:1 co-culture spheroid aggregated from 30k cells seeded in a 96 well plate with round bottom, after 4 days of incubation. Images were acquired on different angles by fixing the spheroid, and placing it in an agarose cube. 

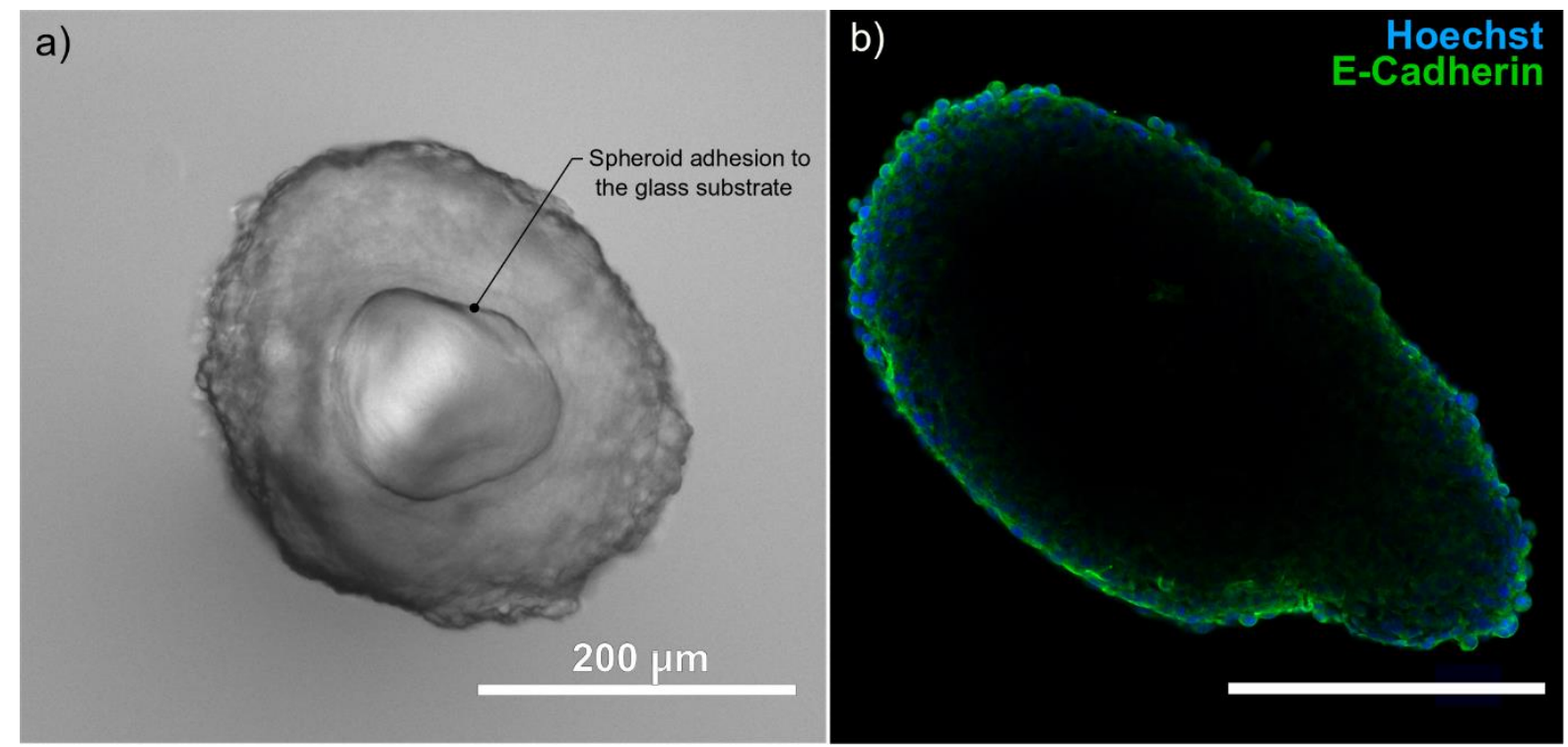

Figure 4-S3 Optimization of VISIKOL protocol. a) Spheroid adhere to glass when methanol evaporation, due to partial hydrophobicity of the glass substrate. b) Spheroid treated with VISIKOL, after 3 days of incubation, and immunostained with E-cadherin antibody and Hoechst. Incomplete evaporation of the methanol prior to mounting in VISIKOL HISTOOM resulted in an uncleared spheroid, with only the outer rim visible.
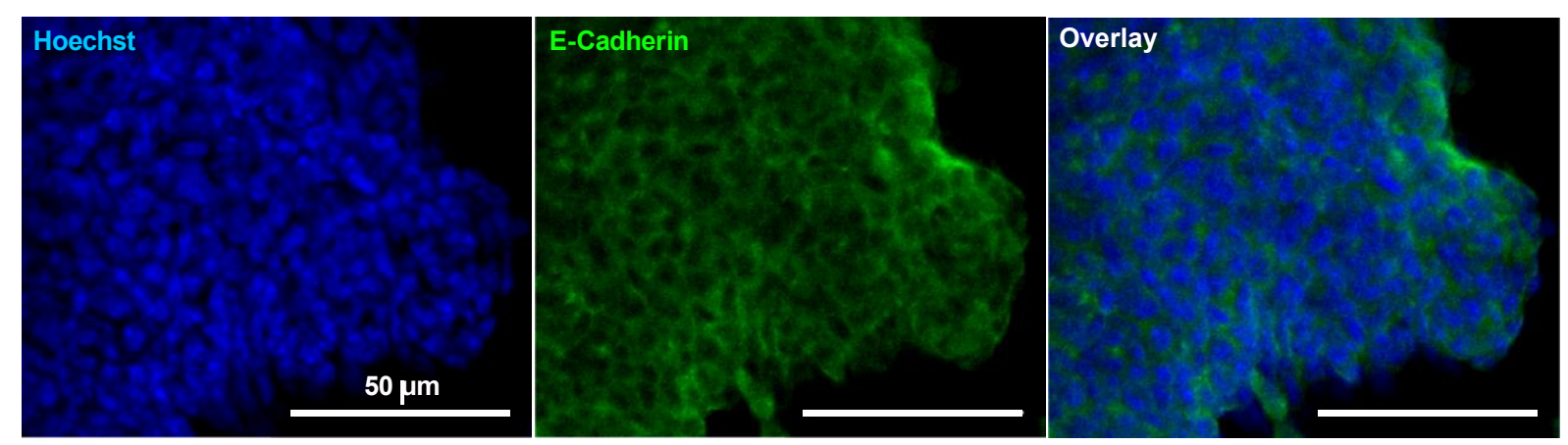

Figure 4-S4 E-Cadherin antibody staining of 4T1 mono-culture spheroids, treated with VISIKOL. Green fluorescent staining is limited to the cell membrane, indicating specific binding of the antibody. 


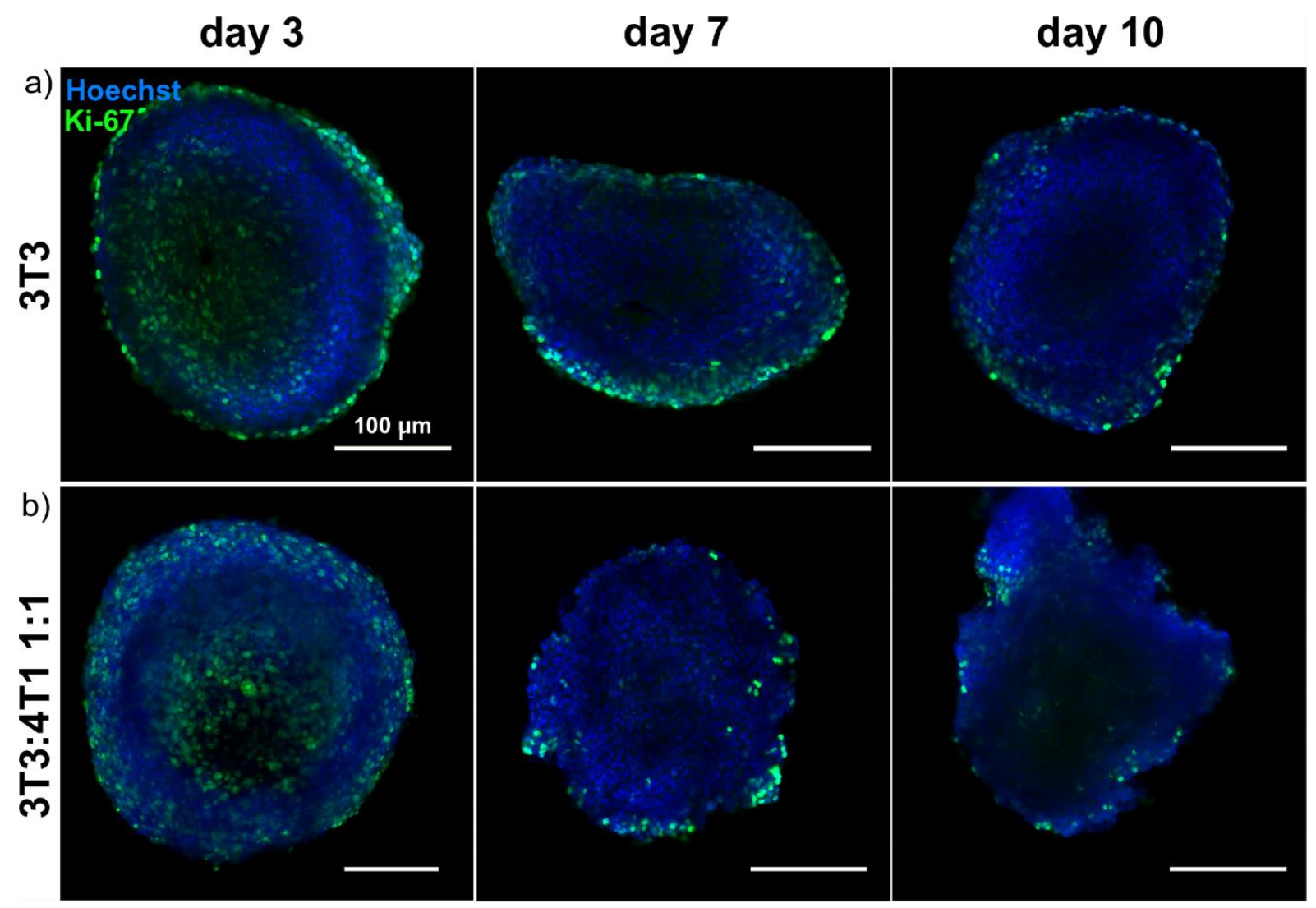

Figure 4-S5 Confocal imaging of (a) 3 T3 mono-culture spheroid and (b) 3T3:4T1 1:1 co-culture spheroid, treated with VISIKOL protocol, after 3, 7, and days in culture. 


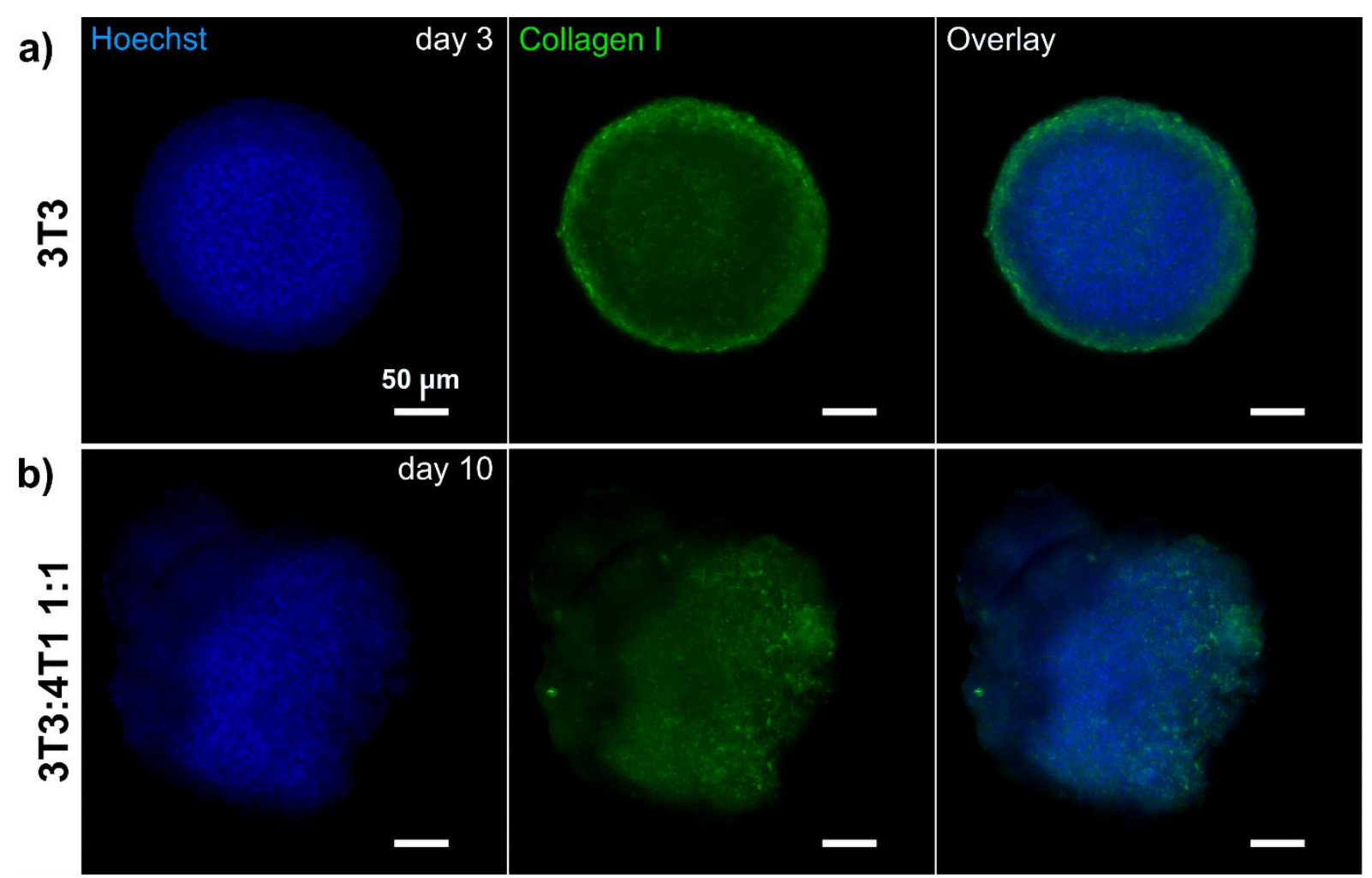

Figure 4-S6 a) $3 T 3$ mono-culture spheroid incubated for 3 days inside the microwell array, treated with VISIKOL, and stained with Hoechst (blue) and collagen I antibody (green). b) 3T3:4T1 1:1 coculture spheroid incubated for 10 days inside the microwell array, treated with VISIKOL, and stained with Hoechst (blue) and collagen antibody (green). 


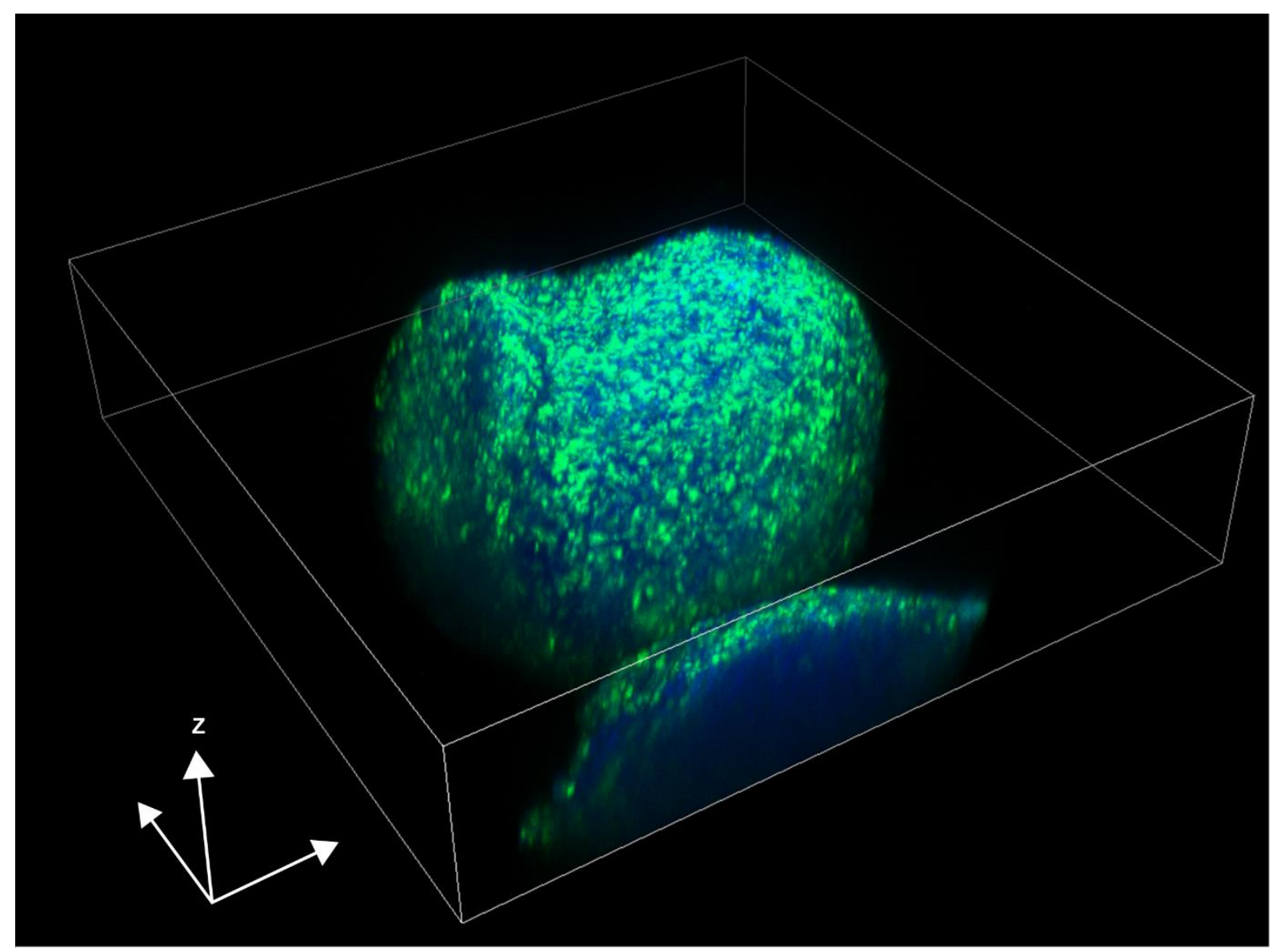

Figure 4-S7 3D reconstruction of a 3T3:4T1 5:1 co-culture spheroid, incubated for 3 days, treated with VISIKOL protocol, and stained with Hoechst (blue) and Collagen I antibody (green). Crosse section of the spheroid along the z-axis confirm the presence of collagen I limited to the edge of the spheroid. 


\title{
Chapter 5 - Tumour-on-a-Chip Platform to Evaluate Nanomedicine Penetration in Co-culture Tumour Spheroids
}

\begin{abstract}
To improve tumour therapies, nanomedicine transport high concentrations of therapeutic drugs directly at the targeted area. The efficacy of this new approach is however affected by the nanoparticle (NPs) properties (e.g., size, charge, molecular composition) as well as the tumour microenvironment composition (e.g., stroma). An additional impact on penetration efficiency recently observed is the level of shear stress present in and around the tumour. This study presents a microfluidic platform able to capture three tumour spheroids, and expose them to NPs under different physiological shear stress. A characterization on the penetration efficiency is performed in situ by investigating the impact of the shear stress in combination other varying parameters: the spheroid stroma content (extra cellular matrix secreted by fibroblasts), and the nanocarrier size (30 nm and $100 \mathrm{~nm}$ ) or material (silica and liposome). Results indicate a significant an increase penetration of small NPs when increasing the shear stress inside the microfluidic device, whereas large NPs remained at the spheroid periphery. The penetration is also strongly reduced in presence of extra cellular matrix in the tissue, and the variation of the chosen nanocarrier material does not impact the penetration depth.
\end{abstract}




\section{INTRODUCTION}

In the last few decades, the field of nanomedicine has made great stride in the development of cancer-targeting therapeutic agents [1]. Whereas traditional chemotherapeutic drugs possess poor water solubility and poor specificity, leading to unfavourable pharmacokinetics and adverse side effects, engineered nanoparticles have given the possibility to selectively target the tumour site while limiting toxicity, resulting into improved drug delivery [2]. These nanoparticle have been continuously improved, by adjusting their physical properties (e.g., material, shape, charge) [3-5] and their targeting capability [6, 7] to ensure successful delivery of the active agents to the target diseased area. As a trade-off, nanoparticles present a much greater size, ranging from a few to a few hundred nanometers, and are therefore presenting new biological challenges, namely extravasation, tissue penetration, and cellular uptake.

To assess the nanoparticle efficiency, pharmaceutical companies currently perform assays on two main platform, the 2D in vitro model and the animal model. On one hand, traditional 2D in vitro tissue culture studies have provided insight on the nanoparticle cellular uptake, but it has long been determined these models were not properly representative of the in vivo physiology [8]. On the other hand, animal in vivo studies have already provided extensive information on nanoparticle extravasation and specificity $[9,10]$ but, besides the ethical concerns, pertinence of the analogy between the animal and human physiologies has always been controversial [11,12]. As a result, alternative in vitro models were developed [13], among which a promising platform has already been heavily investigated for therapeutic assay: the 3D multicellular tumour spheroid (MCTS) [14-17]. This avascular model, prepared through the aggregation of cells, has proven to recapitulate a wide variety of morphological properties (e.g., phenotype change, protein expression profiles) found inside the tumour, due to the presence of strong cell-cell interactions and gradients of oxygen/nutrient [16, 18]. Additionally, the extra cellular matrix (ECM), mainly secreted by fibroblasts, has shown to increase the tissue density, potential reinforcing the barrier to the nanoparticle penetration [19, 20 ]. In previous work, we have demonstrated how the presence of fibroblasts in MCTS acted as a barrier to nanoparticle penetration [21].

Recently, several microfluidic systems were developed, integrating these avascular MCTS, to investigate the interactions (i.e., accumulation, penetration, uptake, toxicity) of nanoparticle 
with these more relevant in vitro models [22, 23]. These studies primarily focused on observing the impact of the nanoparticle and nanomedicine properties on their overall efficacy as nanomedicine models [24-27]. For instance, Albanese et al. developed a microfluidic platform to investigate the impact of nanoparticle size and targeting affinity on their diffusion and retention inside a single MCTS, after which they injected the same nanoparticle in a murine tumour model, yielding comparable results, indicating that nanoparticle larger than $110 \mathrm{~nm}$ could not penetrate the MCTS $[10,28]$. Another interesting physical parameter found to impact the nanoparticle penetration is the shear stress, which in tissues is normally interstitial $(\sim 0.01$ Pa) $[24,29]$. In their work, J. Zhuang et al. previously reported a microfluidic system in which they controlled the shear stress applied on the MCTS to mimic the interstitial condition [29]. However, in vivo the tumour cells can be exposed to a much higher shear stress (i.e., vascular shear stress, 1-7 Pa), especially in late stage cancers where tumour-neighboring capillary vessels become leaky, and tumour cells begin to leave the primary tumour through metastasis [30]. Previous studies have already demonstrated that a small variation in shear stress affected the accumulation of nanoparticles at the tumour site [24].

The objective of this study was to investigate the effect of shear stress exerted on MCTS on the accumulation, penetration and toxicity of nanoparticles in vitro, to better approximate the nanomedicine interaction with the in vivo tumour microenvironment. A dedicated microfluidic system was developed by incorporating three MCTS in a trap, where they were exposed to flow-induced shear stress. An estimation of the flow-rate required to achieve the desired shear stress inside the device was performed using a COMSOL simulation. Next, mouse breast cancer MCTS were exposed to a perfusion of either fluorescent silica Nanoparticle or liposomes, under different flow-rates emulating the shear stress found in vivo (i.e., interstitial, vascular). Based on the work presented in this thesis and in previous work done by us [21], the experiment was performed on a MCTS mono-culture of mouse breast cancer cells. and on MCTS co-culture of cancer cells and fibroblasts, to study the impact of the increased tissue density on the penetration profile. Finally, to further the investigation, several parameters were altered (e.g., nanoparticle size, perfusion time), to ascertain their influence on the nanoparticle accumulation and diffusion inside the MCTS. 


\section{MATERIAL AND METHODS}

\subsection{MICROFLUIDIC DEVICE DESIGN AND FABRICATION}

The design of the microfluidic devices was drawn using Clewin5 software (Phoenix Software, Enschede, The Netherlands). Next, chromium-on-glass masks were fabricated by Delta Mask (Enschede, The Netherlands). A mold was next produced in the NanoLab cleanroom facilities of the MESA+ Institute for Nanotechnology using a standard photolithography process. SU-8 100 (MicroChem, Newton, MA, USA) was spin-coated as two layers of, respectively, 380 and $120-\mu \mathrm{m}$ thickness on a 4-inch silicon wafer $(525 \mu \mathrm{m}$ thick, P-type, $\langle 100\rangle$, single side polished, Okmetic, Helsinki, Finland) and soft-baked for $8 \mathrm{~h}$ in total (first layer pre-bake: $10 \mathrm{~min}$ at $50^{\circ} \mathrm{C} ; 30 \mathrm{~min}$ at $65^{\circ} \mathrm{C}$ and $210 \mathrm{~min}$ at $95^{\circ} \mathrm{C}$; and second layer pre-bake: $20 \mathrm{~min}$ at $50^{\circ} \mathrm{C}, 60 \mathrm{~min}$ at $65^{\circ} \mathrm{C}$ and $200 \mathrm{~min}$ at $95^{\circ} \mathrm{C}$ ). The desired patterns were transferred in the SU-8 negative resin by photolithography through a mask using a mask-aligner (EVG620, EV Group, St. Florian am In, Austria). Next, the wafer was baked again for $2 \mathrm{~h}\left(10 \mathrm{~min}\right.$ at $50^{\circ} \mathrm{C} ; 10 \mathrm{~min}$ at $65^{\circ} \mathrm{C}$; and $50 \mathrm{~min}$ at $75^{\circ} \mathrm{C}$ ) to reinforce cross-linking of the SU-8 photoresist. Uncured resin was removed through 20 min incubation at $20^{\circ} \mathrm{C}$ in 1-methoxy-2-propanol acetate (RER600) developer (Fujifilm, North Kingstown, RI, USA). The resulting mold with the desired SU-8 structures was finally treated with $1 \mathrm{H}, 1 \mathrm{H}, 2 \mathrm{H}, 2 \mathrm{H}$-perfluorodecyltrichlorosilane (FDTS, abcr GmbH, Karlsruhe, Germany) in the vapor phase to make the surface hydrophobic and facilitate unmolding of the PDMS structures.

Polydimethylsiloxane (PDMS) was prepared by mixing a silicone pre-polymer and curing agent (SYLGARD 184, Dow Corning, Midland, MI, USA) in a 10:1 weight ratio. This mixture was degassed under vacuum, poured on the SU-8-on-silicon mold and cured for $3 \mathrm{~h}$ at $60^{\circ} \mathrm{C}$. The resulting PDMS fluidic layer was peeled off the mold and reservoirs were created with a 1-mm diameter puncher (Miltex GmbH, Rietheim-Weiltheim, Germany) in the PDMS to create inlets and outlets.

PDMS devices were assembled with a glass coverslip coated with a thin layer of PDMS $(20 \mu \mathrm{m})$. To that end, a PDMS mixture (10:1 weight ratio prepolymer: curing agent) was evenly spread with a roller on a \#1 glass coverslip ( 22 x 40 mm, VWR, Radnor, USA), and subsequently cured at $60^{\circ} \mathrm{C}$ for $2 \mathrm{~h}$. Both the PDMS fluidic layer and the PDMS-coated coverslip were exposed to plasma treatment (CUTE plasma cleaner, Femto Science Inc., Gyeonggi-Do, Korea) for $45 \mathrm{~s}$ at $510^{-1} \mathrm{mT}$ Trr, and assembled together. No final baking step was performed to prevent the PDMS 
from deactivating, leading to the formation of bubbles when injecting solutions. Finally, the microfluidic channels were filled with a filtered aqueous 1\% w/w Pluronic F-127 solution (SigmaAldrich, Zwijndrecht, The Netherlands).

\subsection{COMSOL SimULATION}

Simulations were conducted using COMSOL Multiphysics (V5.1) software (Burlington, MA, USA) to evaluate the flow and shear stress profiles in the microfluidic systems and in the vicinity of the tumour spheroids. To that end, a 3D model of the microfluidic device was drawn on scale, with three 300- $\mu$ m diameter spheroids trapped in the microchamber. Spheroids were treated here as hard particles. A constant flow of liquid set with water properties at $37^{\circ} \mathrm{C}$ (density of $0.9933 \mathrm{~g} \mathrm{~cm}^{3}$ and dynamic viscosity of $0.6913 \mathrm{mPa}$ ) was applied at a flowrate of $0.1,1$ or $10 \mu \mathrm{L} \mathrm{m^{-1 }}$ throughout the channel using the "Laminar flow" Physics COMSOL package. Using the in-software shear rate function, a display of the shear rate was projected on the surface of each spheroid. By multiplying the profile with the dynamic viscosity of solution (here water), we obtained a display of the shear stress on each spheroid surface. The values of shear stress from each simulation in all condition was extracted from the front end of the spheroid facing the flow.

Next, the trajectory and accumulation of nanoparticles was evaluated in a second simulation in the same microfluidic device with three MCTS, still using COMSOL Multiphysics (V5.1) and the "Particle tracking for Fluid Flow" package, with a Brownian motion. Here, a plug of 3000 hard NPs (100-nm diameter) was injected upstream in the channel, with an initial velocity of $10 \mu \mathrm{L} \mathrm{min}{ }^{-1}$, and their motion was modeled using the Navier-Stokes equation and, as before, a laminar flow.

\subsection{MICROFLUIDIC SETUP}

Flows in the microfluidic devices were actuated and controlled with a Harvard syringepump 11e (Harvard Apparatus, Holliston, MA, USA). For each experiment, two independent devices were connected in series. All connections between the devices and to the syringe used Tygon Microbore Tubing (0.02 in inside diameter x 0.060 in outside diameter, Cole-Parmer, Chicago, IL, USA) of $30 \mathrm{~cm}$ in length between the syringe pump and the first microfluidic device and ca. $5 \mathrm{~cm}$ between the devices. To connect the tubing to the devices, 0.9-mm metallic fitting were cut blunt from syringe needles (BD Biosciences, Franklin Lakes, NJ, USA) and inserted 
inside the tubing as connectors to the microfluidics device inlets and outlets. A 20 -mL plastic syringe (BD) equipped with a $0.2-\mathrm{mm}$ diameter filter at its outlet tip was filled with the solutions to be injected in the devices, and placed on the Harvard Apparatus Syringe pump. A magnet was introduced inside the syringe and a magnetic stirrer placed below the syringe to prevent sedimentation of the nanoparticles in the syringe. The outlet of the system was connected to a waste container. The microfluidic devices were placed in a standard cell culture incubator $\left(37^{\circ} \mathrm{C}\right.$, $\left.5 \% \mathrm{CO}_{2}\right)$.

\subsection{Cell Culture}

4T1 mouse breast cancer cells and 3T3 mouse fibroblasts were obtained from the American Type Culture Collection (ATCC, Rockville, MD, USA). 4T1 and 3T3 were cultured, respectively, in Roswell Park Memorial Institute (RPMI) 1640 medium and Dulbecco's Modified Eagle's Medium (DMEM), both media being supplemented with $2 \mathrm{mM}$ L-glutamine, $10 \%$ fetal bovine serum (FBS) and antibiotics $\left(50 \mathrm{U} \mathrm{mL}^{-1}\right.$ Penicillin and $50 \mathrm{ng} \mathrm{mL}^{-1}$ streptomycin). Cells were expanded in 75- $\mathrm{cm}^{2}$ culture flask (CELLSTAR ${ }^{\circledR}$ Filter Cap 75- $\mathrm{cm}^{2}$, Greiner Bio-One, Kremsmünster, Austria) in an incubator under standard humidified conditions $\left(37^{\circ} \mathrm{C}, 5 \% \mathrm{CO}_{2}\right)$ and medium was refreshed every 2 days. Briefly, culture medium was aspired from the culture flasks and cells washed with $10 \mathrm{~mL}$ of sterile PBS 1X buffer (Lonza, Basel, Switzerland) to remove dead (floating) cells. $2 \mathrm{~mL}$ of a trypsin-EDTA (0.25\%) solution (Thermo Fisher Scientific, Waltham, MA, USA) was added for $5 \mathrm{~min}$ to detach the cells from the surface. The cells were next re-suspended in $8 \mathrm{~mL}$ of their respective supplemented medium to block the action of the trypsin and collected in $10 \mathrm{~mL}$ tubes. After centrifugation at $300 \mathrm{~g}$ for $3 \mathrm{~min}$, medium was removed and $2 \mathrm{~mL}$ of fresh DMEM medium was added. Cells were counted, and $c a .2$ million cells were seeded in new culture flasks. The remaining cells were kept for further experiments, as described in the following section.

\subsection{MCTS PREPARATION}

Multi-cellular tumour spheroids (MCTS) were prepared as previously reported by us, using either only 4T1 breast tumour cells (mono-culture MCTS) or a 5:1 mixture of 3T3 fibroblasts and 4T1 breast tumour cells (co-culture MCTS). ${ }^{12,29}$ An array of microwells (400- $\mu \mathrm{m}$ diameter and 200- $\mu \mathrm{m}$ depth) was prepared in a 35-mm diameter Petri dish (Greiner Bio-One) using soft-embossing and a PDMS mold. The PDMS mold was prepared as the microfluidic devices using a SU-8-on-silicon mold. ${ }^{29}$ The soft-embossed Petri dishes were sterilized with a $70 \%$ ethanol solution and coated with sterile $1 \%$ w/w Pluronic F-127 overnight. After removal of 
the Pluronic solution, $2 \mathrm{~mL}$ of DMEM medium supplemented with $\mathrm{ca}$. 2.5 million cells was added in the Petri dish. Cells were forced into the microwells by centrifugation (600 g for $5 \mathrm{~min}$ ) with a slow deceleration to prevent cells from escaping the microwells. The excess of cells outside the microwells was removed by carefully washing the Petri dish three times with $2 \mathrm{~mL}$ PBS, before addition of $2 \mathrm{~mL}$ of supplemented DMEM medium for culture. The Petri dishes with the cells were kept under standard culture conditions in a humidified incubator $\left(37^{\circ} \mathrm{C}, 5 \% \mathrm{CO}_{2}\right)$. After typically $48 \mathrm{~h}$ incubation, the resulting spheroids were collected in an Eppendorf microtube (Eppendorf GmbH, Hamburg, Germany) in fresh DMEM medium for further experimentation.

\subsection{NANOPARTICLE DELIVERY EXPERIMENTS}

Fluorescently-labelled silica nanoparticles (Sicastar®-redF-COOH (size of $30 \mathrm{~nm}$, excitation: $569 \mathrm{~nm}$, emission: $585 \mathrm{~nm}$ ) and Sicastar®-greenF-COOH (size of $100 \mathrm{~nm}$, excitation: $485 \mathrm{~nm}$, emission: $510 \mathrm{~nm}$ ) (Micromod Partikeltechnologie GmbH, Rostock, Germany) were suspended in serum-free DMEM medium at a final concentration of $100 \mu \mathrm{g} \mathrm{mL} \mathrm{m}^{-1}$. This solution was introduced in a syringe as described previously, and injected in the microfluidic devices at a flowrate of $0.1,1$ or $10 \mu \mathrm{L} \mathrm{min}{ }^{-1}$ for 24 or $48 \mathrm{~h}$.

The same experiment was repeated with 100-nm diameter liposomes stained with rhodamine dye. Liposomes were prepared using the ethanol injection method as described previously ${ }^{39}$ An alcoholic solution of DPPC (dipalmitoyl phosphatidylcholine), cholesterol, PEG(2000)-DSPE (both from Lipoid Gmbh, Ludwigshafen am Rhein, Germany) was added together with DHPE-Rhod (dihexadecanoyl-sn-glycero-3-phosphoethanolamine-Rhodamine) in aqueous solution, to yield a molar ratio of 1.88:1.00:0.15:0.02 DPPC:cholesterol:PEGDSPE:DHPE-Rhod. The resulting lipid dispersion was sequentially extruded at $60^{\circ} \mathrm{C}$ using a medium pressure extruder (Lipex, Northern lipids, Burnaby, Canada) equipped with two stacked polycarbonate membrane filters with $100 \mathrm{~nm}$ pores. The resulting liposome solution was purified by tubing dialysis $(100 \mathrm{kD})$ against PBS to yield a homogeneously sized suspension $(100 \mathrm{~nm})$.

\subsection{VIABILITY ASSAY}

Cell viability was evaluated using a live/dead assay and Calcein AM and Ethidium homodimer-1 (EthD-1) (both Thermo Fisher Scientific). As before, three spheroids were placed in each microfluidic device and exposed to a continuous flow $\left(0.1,1\right.$ or $\left.10 \mu \mathrm{L} \mathrm{min}{ }^{-1}\right)$ of nonfluorescent nonporous silica NPs (Sicastar-COOH, $100 \mathrm{~nm}$ and $30 \mathrm{~nm}$ in size, Micromod Partikeltechnologie $\mathrm{GmbH}$ ) at a final concentration of $100 \mu \mathrm{g} \mathrm{mL}^{-1}$ in serum-free DMEM medium 
for $48 \mathrm{~h}$. After exposure to the NP suspension, a mixture of Calcein AM (4 $\mu \mathrm{M})$ and Ethidium homodimer-1 $(2 \mu \mathrm{M})$ was manually pipetted twice in the devices. As a negative control, MCTS were exposed to a $10 \mu \mathrm{L} \mathrm{min}{ }^{-1}$ flow of serum-free DMEM without silica nanoparticles. Cell viability results were obtained by measuring the green signal (live cells) and red signal (dead cells) of each pixel on the images, and calculated as a percentage of living cells in the MCTS, following the following equation:

Viability $=\frac{\sum_{\mathrm{i}=1}^{\mathrm{N}} \mathrm{x}_{\mathrm{i}}}{\mathrm{N}} * 100$

with $N$ the total number of pixels in which the intensity of live or dead cells is above a background noise threshold value, and $x_{i}$ a Boolean numerator of the pixel $i$ where $x_{i}=1$ if the live signal (green) intensity is superior to the dead signal (red) intensity, and $x_{i}=0$ otherwise. The background threshold value as calculated in each image by measuring the average signal in a dark area present near the MCTS. Each measurement was performed on three spheroids present in each microfluidic device and experiments were performed three times per condition (three independent devices), with a total of 9 MCTS per condition.

\subsection{CONFOCAL MICROSCOPY IMAGING AND DATA ANALYSIS}

The NP penetration was evaluated using confocal microscopy imaging (Nikon A1RA1, Tokyo, Japan). All images were acquired with a 20x air objective and an image thickness of $\sim 20.4 \mu \mathrm{m}$ according to the confocal software (NIS-Elements, Nikon). For each spheroid, a single picture was acquired directly in the microfluidic device at $c a$. the half-height of the spheroid. Green fluorescent NPs were excited using a $488 \mathrm{~nm}$ argon laser and imaged using the Alexa Fluor 488 settings. Red fluorescent NPs were excited using a $561 \mathrm{~nm}$ diode pumped solid-state laser and imaged using the Alexa Fluor 568 settings. In 3D confocal microscopy, light scattering inside the tissue can become a possible limitation to the imaging deep inside the tissue, but, with the size of the MCTS $(\sim 300 \mu \mathrm{m})$ and the confocal objective used, the impact of the light scattering was considered negligible, as confirmed by the observed penetration of the NPs reaching almost the core the MCTS in some conditions,

For each spheroid, the nanoparticle penetration profile was quantified using an in-house MATLAB script, described in supplementary information, with Figure S1. The image processing resulted in a local penetration profile inside a single spheroid. This penetration profile was integrated as a function of the distance from the spheroid edge to depict the total penetration 
profile from the edge of the spheroid to a certain radial position, this reflecting the total amount of nanoparticles delivered to the spheroid in the "shell" between its edge and this specific radial position. The total relative fluorescence intensity was normalized, $100 \%$ being the value reached at the plateau of this resulting curve. The data was then averaged from 6 spheroids, from 3 different set of experiment. The nanoparticle maximum penetration depth was defined by $\sigma$ as the radial position value corresponding to $95 \%$ of this relative integrated fluorescence intensity.

\subsection{Statistical ANALYSIS}

The spheroid size, penetration depth, and viability assay data were subjected to statistical analysis by performing a one-way ANOVA test, using the Tukey method, with different significance levels (p-value).

\section{RESUlTS \& DisCUSSION}

\subsection{TUMOUR-ON-A-CHIP PLATFORM}

As in previous work, we considered mouse breast tumour models for further possible comparison with in vivo studies, and we prepared two types of MCTS: mono-culture spheroids solely comprising 4T1 murine breast cancer cells; and co-culture spheroids combining 3T3 murine cancer-associated fibroblasts and 4T1 cells to study the impact of the ECM produced by the fibroblasts on the nanomedicine delivery. ${ }^{12}$ In the co-culture MCTS, these two cell types were introduced in a 3T3:4T1 5:1 ratio, as identified in our previous study as most representative of the tumour microenvironment in terms of ECM production and expression of the stromal marker $\alpha$-SMA (alpha-Smooth Muscle Actin). ${ }^{12}$ MCTS were prepared in a previously reported microwell array (Figure 5-1A), soft-embossed in a Petri dish, and coated with a $1 \%$ Pluronic F-127 solution to render the surface cell-repellent and to promote cell aggregation. ${ }^{29}$ All MCTS in this work were prepared in microwells of $400 \mu \mathrm{m}$ diameter and $200 \mu \mathrm{m}$ height, and the resulting spheroids exhibited homogeneous sizes of $273 \pm 28 \mu \mathrm{m}$ and $373 \pm 28 \mu \mathrm{m}$, for the mono- and co-culture MCTS ( $<<0.001)$, respectively, after $48 \mathrm{~h}$ of culture in the microwell arrays (Figures 5-1B and 5-1C). Noteworthy, MCTS generated in our microwell arrays were not spherical, due to the cylindrical shape and flat bottom of the 
microwells, but rather oblate, and they typically had a height of $c a .210 \mu \mathrm{m}$, which is much smaller than their diameter.
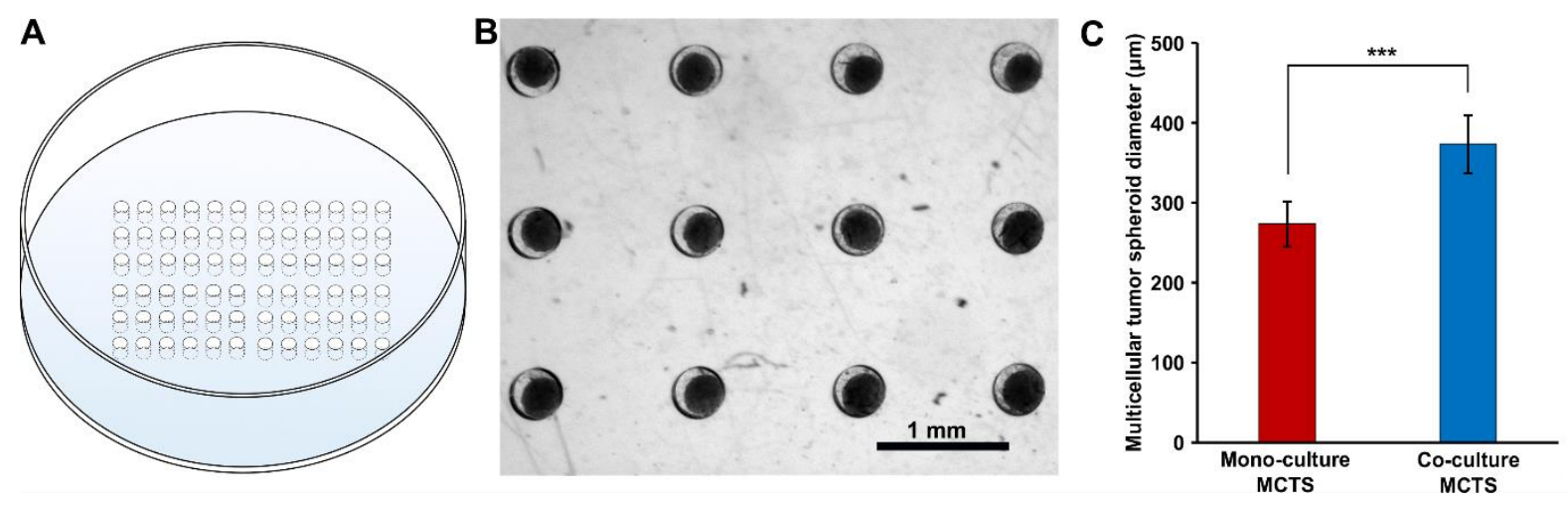

Figure 5-1 Multicellular tumor spheroid (MCTS) formation A) Schematic representation of a microwell array soft-embossed in a polystyrene Petri dish, with microwells of $400 \mu \mathrm{m}$ in diameter and $200 \mu \mathrm{m}$ in depth (not to scale). B) Microscopic pictures of co-culture MCTS in part of a microwell array, after 48 $h$ of culture in the microwell array. C) MCTS diameter for both mono-culture (4T1 only) and co-culture spheroids (5:1 3T3:4T1) after 48 h of culture in the microwell array $\left(^{* * *} p<0.01\right.$, number of spheroids analyzed per condition $n=9$ ).

MCTS were introduced in a dedicated microfluidic device to yield a tumour-on-a-chip platform. The microfluidic device consisted of one circular chamber ( $3 \mathrm{~mm}$ diameter; $500 \mu \mathrm{m}$ height) equipped with a row of pillars $(260 \mu \mathrm{m}$ in width, separated by $100 \mu \mathrm{m})$ with triangular cross-section placed in its center to trap several spheroids next to each other (Figure 5-2A). A triangular cross-section was chosen here to guide the spheroid between two pillars, and prevent spheroids from being in contact between one another. Here, three $c a$. 300- $\mu$ m size spheroids were simultaneously introduced in the chamber, while taking care not to have any contact between them not only to prevent them from merging but also to maintain a flow between them (Figure 5-2B). In this configuration, all spheroids were exposed to the same flow conditions and received the same amount of nanomedicines, as confirmed by COMSOL simulations (vide infra). The device was fabricated from polydimethylsiloxane (PDMS) and bonded with a thin glass substrate for in situ imaging using confocal microscopy. The entire device was treated with a $1 \% \mathrm{w} / \mathrm{w}$ Pluronic F-127 solution to prevent the spheroids from attaching and spreading on the bottom substrate. Since Pluronic F-127 better coats PDMS than glass, the glass substrate was coated with a thin and homogeneous layer of PDMS (ca. $20 \mu \mathrm{m})$, before assembly of the two substrates. 
A

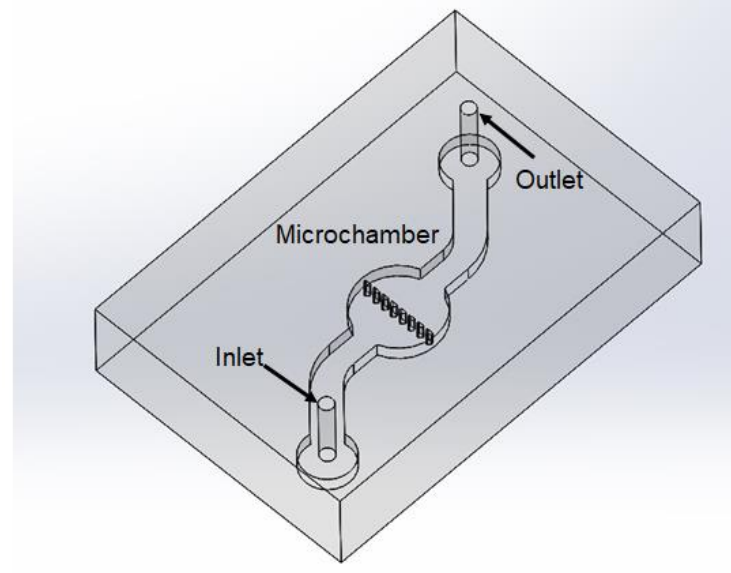

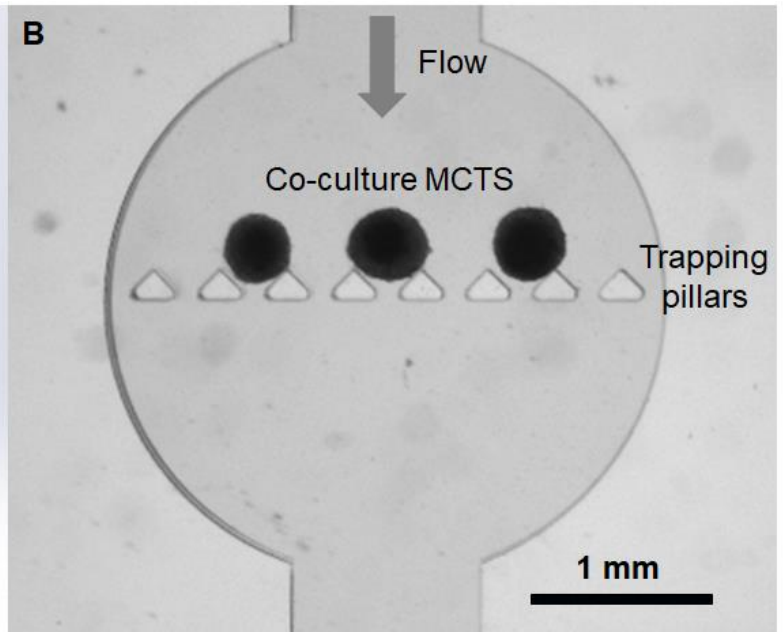

Figure 5-2 Microfluidic device for studying the NP delivery in multicellular tumor spheroids. A)

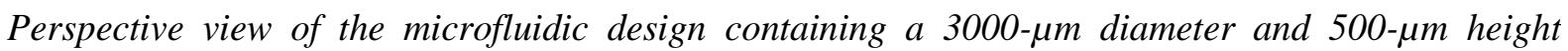
microchamber equipped with a row of pillars (spacing $100 \mu \mathrm{m}$; width $260 \mu \mathrm{m}$ ) to trap MCTS in the middle of the chamber; B) Microscopic picture showing three co-culture MCTS loaded in the microfluidic device and trapped in the microchamber against the row of pillar.

\subsection{COMSOL SIMULATIONS}

Before starting the NPs exposure experiments, two series of simulations were performed: to investigate the flow-induced shear stress the MCTS would be exposed to in the device, and to model the nanomedicine-MCTS interactions. Both series of simulations were conducted using COMSOL Multiphysics ${ }^{\mathrm{TM}}$.

\subsubsection{Flow-induced shear stress}

First, in a 3D simulation, a device with the aforementioned design and dimensions was considered with three regularly spaced 300- $\mu$ m diameter spheroids (Figure 5-3A). MCTS were modeled as spherical objects in a first approximation. The MCTS were treated as solid, nonporous, non-adherent, incompressible and hard objects. Continuous medium perfusion was applied and modeled using the "laminar flow" package. Here, we decided to include different flow conditions to mimic configurations found in vivo. The highest shear in vivo is the vascular shear stress, which is induced by blood flow in blood vessels and, the larger the blood vessel the higher the associated shear stress; in veins this shear stress is of 0.1-0.5 Pa, and in aorta of 1-7 $\mathrm{Pa} .{ }^{30}$ The lowest shear stress, or interstitial shear stress, which is found inside the tissues outside the vascular system, varies with the location in the body and the cellular composition, and typically ranges between 0.001 and $0.01 \mathrm{~Pa}^{31}$ 
The amount of shear stress exerted on a surface directly relates to the flow velocity of fluid in proximity of this surface, as well as the viscosity of this fluid. To model the entire aforementioned range of shear stress on the spheroid surface in our device, we varied the flow of the perfusion (Figure 5-3). According to the simulation, all spheroids were roughly exposed to the same shear stress pattern. Using a flow-rate of $10 \mu \mathrm{L} \mathrm{min}{ }^{-1}$, we found a shear stress between $0.1 \mathrm{~Pa}$ and $c a .0 .5 \mathrm{~Pa}$ in the front and at a $45^{\circ}$ angle, both at the half-height of the spheroid (Figure 5-3A and 5-3B), and this for all 3 spheroids. These results first indicate that a flow-rate of $10 \mu \mathrm{L} \mathrm{min}{ }^{-1}$ faithfully mimics shear stress conditions found in veins in vivo.

We next varied the flow-rate in the COMSOL simulations to mimic the interstitial shear stress, which is typically 2 to 3 orders of magnitude lower. As expected, using a $0.1 \mu \mathrm{L} \mathrm{min}{ }^{-1}$ flow-rate, the shear stress exerted on the spheroid on the same positions as before, varied between 0.01 and $0.05 \mathrm{~Pa}$. Based on these simulations, we decided to utilize similar flow-rate values experimentally to approximately reproduce vascular $\left(10 \mu \mathrm{L} \mathrm{min}^{-1}\right)$ and interstitial shear stresses $\left(0.1 \mu \mathrm{L} \mathrm{min}^{-1}\right)$, and to include as well a third intermediate flow-rate value of $1 \mu \mathrm{L} \mathrm{min}-$ 1 .

\subsubsection{Nanoparticle transport}

In a second series of simulations, we evaluated the delivery of $100 \mathrm{~nm}$ nanoparticles to the three MCTS captured in the device. Here we used a 2D simulation, selected at mid height of the spheroid height. At the start of the simulation, a plug of 3000 nanoparticles modeled as hard spheres with a density of $2650 \mathrm{~kg} \mathrm{~m}^{3}$, was injected in the device, using the "Particle Tracking" package, at one of the three aforementioned flow-rates.

The simulations first confirmed the parabolic character of the flow with a significant dispersion of the nanoparticle plug in the 2-mm long inlet channel (Figure 5-3B). At a $10 \mu \mathrm{L}$ $\min ^{-1}$ flow-rate, the nanoparticle plug reached the spheroids after $8 \mathrm{~s}$, and passed them in $12 \mathrm{~s}$ (Figure 5-3). Decreasing the flow-rate to 1 and $0.1 \mu \mathrm{L} \mathrm{min}^{-1}$ correspondingly increased these time values to 80 and $120 \mathrm{~s}$, and 800 and $1200 \mathrm{~s}$, respectively. In all conditions (30 nm and 100 nm NPs), out of the 3000 nanoparticles, $c a .65$ nanoparticles were found in contact with each spheroid in the device, which roughly correspond to a nanoparticle delivery efficiency to the MCTS of $6.5 \%$. The rest, and thus the majority, of the nanoparticles exited the system through the outlet in this configuration, where we neglected interactions between the nanoparticles and other surfaces in the microfluidic device. Moreover, the spheroid is in theory maximum 300 $\mu \mathrm{m}$ tall, whereas the channel is $500 \mu \mathrm{m}$. We therefore expected a large number of NPs were 
transported above the MCTS, and the actual delivery efficiency to be even lower. Due to this theoretical low percentage of NPs retained in the device, it was estimated that several devices could be placed in series for the experiments.
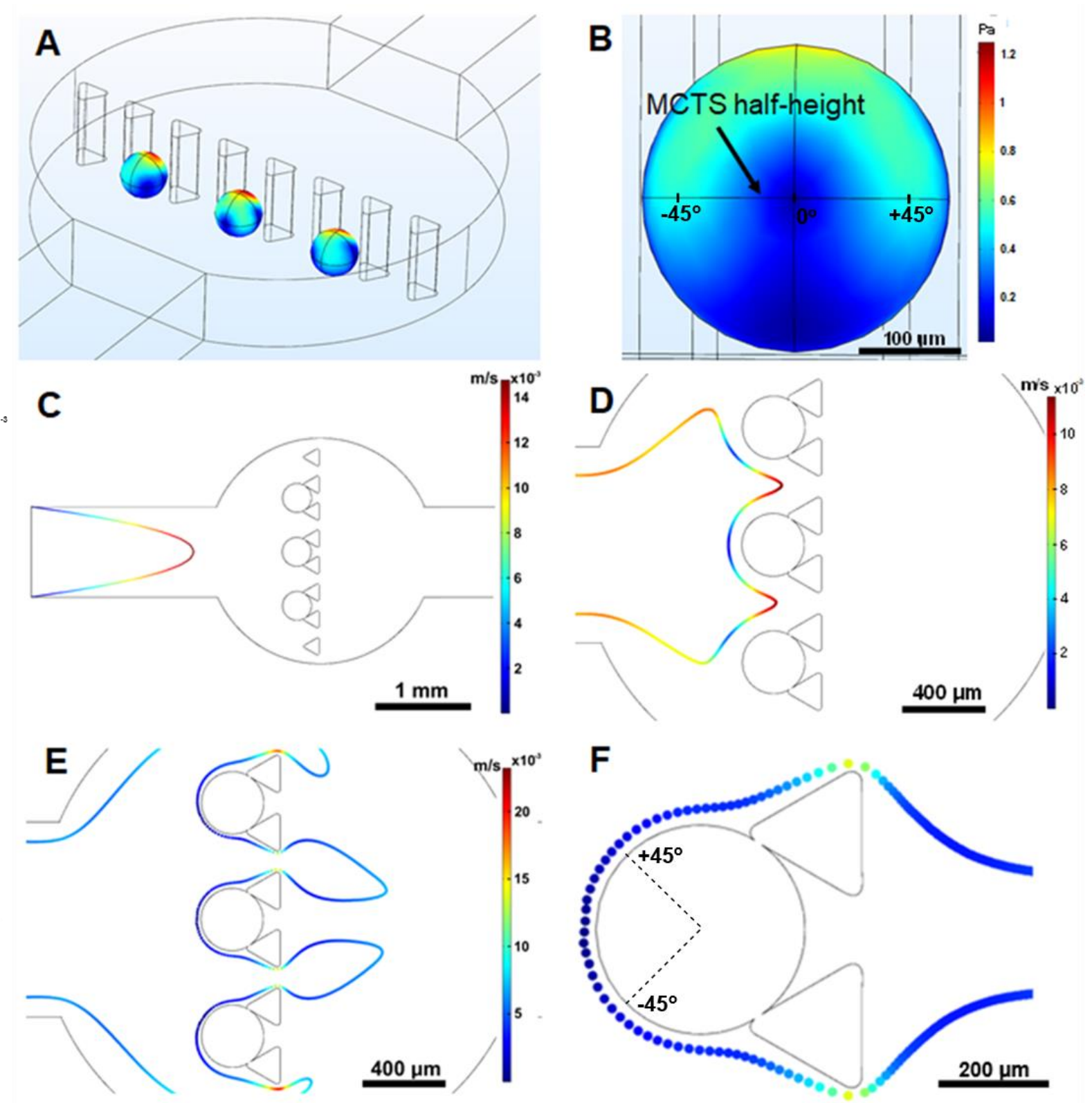

Figure 5-3 COMSOL MultiPhysics simulation of the flow in the device and of the nanoparticle delivery. $A \& B)$ Wall shear stress profile on three MCTS in the microfluidic device, exposed to a constant flowrate of $10 \mu \mathrm{L} \mathrm{min}{ }^{-1}$. A) Perspective view of the entire microchamber; B) Enlarged and frontal view of one MCTS showing the existence of a maximum shear stress on the top of the spheroid and two positions of higher shear stress at about $+45^{\circ}$ and $-45^{\circ}$ at its half-height. $C$ to F) Delivery of a plug of 3000100 $n m$ diameter NPs to three spheroids trapped in a microfluidic device, at timepoint $(C) t=4 s,(D) t=8$ $s,(E, F) t=12 \mathrm{~s}$, at a constant flow-rate of $10 \mu \mathrm{L} \mathrm{min}^{-1}$, showing the velocity profile of the NP plug with different enlargement factors. 


\subsection{NANOPARTICLE-MCTS INTERACTIONS}

\subsubsection{MCTS exposure to model silica nanoparticles.}

As a next step, MCTS, based on either a mono- or a co-culture and trapped in the microfluidic device were exposed to a continuous flow of nanoparticles, used here as surrogates for nanomedicines. As a first model, commercially available fluorescent silica nanoparticles were utilized, since they are easily tracked to quantify their penetration in the MCTS. These nanoparticles were $30 \mathrm{~nm}$ and $100 \mathrm{~nm}$ in size; respectively, labeled with red and green fluorophores; non-porous and both carboxy-terminated (zeta potential $-40 \mathrm{mV}$ ). These two types of nanoparticles were diluted in serum-free DMEM medium at a concentration of $100 \mu \mathrm{g}$ $\mathrm{mL}^{-1}$ each, as in previous work [1]. Serum-free medium was employed here to ensure no protein corona would form, which would influence the nanoparticle physical properties (size \& charge), and in turn the nanoparticle-MCTS interactions and their penetration in the MCTS [2]. The nanoparticle suspension was continuously perfused in the microfluidic devices for 24 or $48 \mathrm{~h}$ in a standard incubator $\left(37^{\circ} \mathrm{C}\right.$ and $\left.5 \% \mathrm{CO}_{2}\right)$, two devices being connected in series to one syringe. As mentioned before, the flow-rate was set to $0.1,1$, or $10 \mu \mathrm{L} \mathrm{min}{ }^{-1}$. The nanoparticle penetration was assessed on-chip using confocal microscopy, a single image being acquired per MCTS at about its half-height. The penetration of the nanoparticles in the MCTS was quantified using an in-house written MATLAB script, as detailed in Supplementary materials. Briefly, and as depicted in Figure 5-4A, the MCTS section on the image was assimilated to a circle, and for every radius of this circle the nanoparticle penetration was quantified from the edge of the spheroids towards its center. In this work, we limited this quantitative study to one third of the MCTS, which was deemed to be the most relevant section of the tissues. First, the nanoparticle penetration profile was plotted in polar coordinates for this $120^{\circ}$-wide considered section (Figure 5-4B), presenting the variation in penetration along the angular profile. Interestingly, the 6-fold increase in the shear stress predicted from the simulation (Figure 53B) between the front of the MCTS and the sides at a $45^{\circ}$ angle, was not found in our experiments in terms of nanoparticle accumulation and penetration. Next, for each spheroid, the fluorescence intensity profiles were averaged in this $120^{\circ}$ angular range and plotted as a nanoparticle distribution profile, and as relative fluorescence intensity values as a function of the radial distance from the edge of the MCTS towards their center (Figure 5-4C). Finally, the profile was integrated as a function of the distance from the spheroid edge and normalized, to yield an integrated nanoparticle delivery profile (Figure 5-4D), from which a maximum depth of penetration $\sigma$ was extracted. The maximum depth was defined as the deepest position 
95\% of the nanoparticles penetrated inside the MCTS. This particular value was selected to eliminate experiment- and measurement-induced fluctuations.

The same data analysis was repeated for all tested conditions: mono- and co-culture spheroids, 30- and 100-nm nanoparticles, flow-rates of $0.1,1$ and $10 \mu \mathrm{L} \mathrm{min}^{-1}$, and $24 \mathrm{~h}$ and $48 \mathrm{~h}$ continuous exposure. For each tested condition, data were averaged for six independent spheroids extracted from three separate experiments. The maximum of penetration $\sigma$ for each set of experiments are summarized in Table 5-S1.

Figure 5-5 presents representative confocal microscopy pictures of MCTS in the microfluidic device after their exposure to the continuous flow of nanoparticles for $48 \mathrm{~h}$ (Figure 5-5A), together with the nanoparticle distribution and integrated penetration profiles (Figures 5-5B \& C, respectively), for the three different flow-rate values. First, at a low flow-rate $(0.1$ $\left.\mu \mathrm{L} \min ^{-1}\right)$, the nanoparticle distribution profiles revealed no significant impact of the nanoparticle size and the MCTS cellular composition on the nanoparticle delivery, and for all four series of experiments, accumulation occurs at the MCTS edge, with most of the NPs present at less than $20 \mu \mathrm{m}$ from the surface. At a 10 time higher flow-rate, the shape of this distribution profile changed with the increase in penetration of a small number of nanoparticles towards the center of the MCTS, revealing not only accumulation of the nanoparticles at the edge of the MCTS but also penetration into the tissues. Noteworthy, this penetration is more marked for mono-culture than co-culture MCTS, and slightly greater for the smaller nanoparticles $(30 \mathrm{~nm})$ in mono-culture MCTS, showing an influence of both the cellular composition and the nanoparticle size for mono-culture MCTS. These nanoparticle distribution profiles were significantly different at an even higher flow-rate of $10 \mu \mathrm{L} \mathrm{min}{ }^{-1}$, with again a clear impact of both the cellular composition and the nanoparticle size. Specifically, for the 100-nm nanoparticles, a striking influence of the cellular composition was found: the co-culture MCTS presented roughly the same nanoparticle distribution profile as at a $1 \mu \mathrm{L} \mathrm{min}{ }^{-1}$ flowrate, while mono-culture MCTS allowed more penetration. In contrast, no clear impact of the cellular composition of the MCTS was found for the 30-nm nanoparticles; they accumulated across a thicker layer $(\mathrm{ca} .50 \mu \mathrm{m})$ at the tissue edge and penetrated much deeper in the tissues $(>100 \mu \mathrm{m})$. After $24 \mathrm{~h}$ perfusion only (Figure 5-S2), these nanoparticle distribution profiles were qualitatively very similar for the 0.1 and $1 \mu \mathrm{L} \min ^{-1}$ flow-rates, with yet a greater difference in penetration in the mono-culture MCTS between the 30- and the 100-nm 

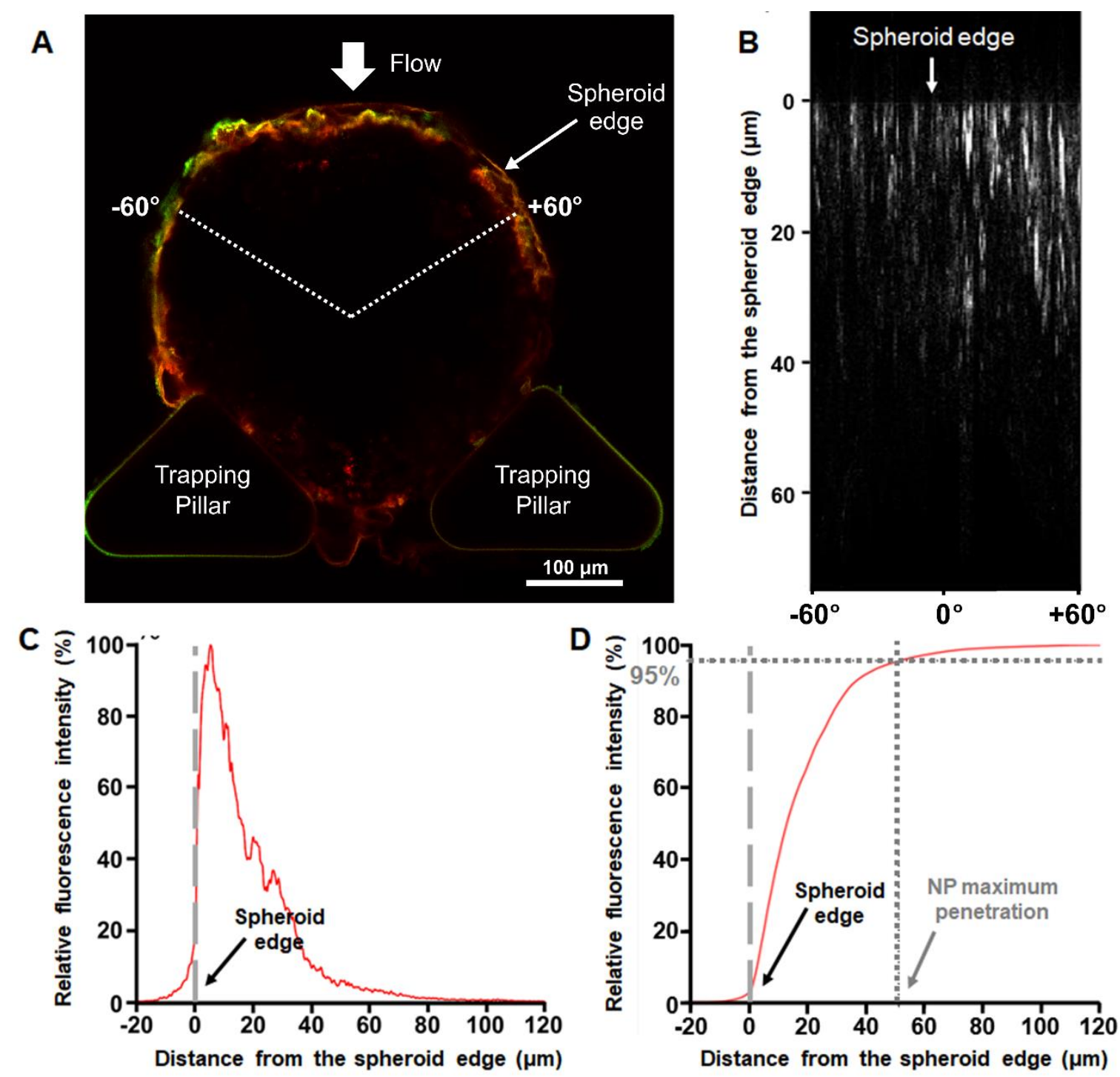

Figure 5-4 Quantification of the NP penetration in MCTS in the microfluidic device using confocal microscopy imaging and data analysis. A) Fluorescence microscopy picture of one mono-culture MCTS in the microfluidic device after $48 \mathrm{~h}$ continuous perfusion of the nanoparticle suspension at $10 \mu \mathrm{L} \mathrm{min}$ ${ }^{l}$, showing the considered angular range for data analysis. B) Corresponding 30-nm red nanoparticle penetration profile in the same MCTS represented in polar coordinates over the considered angular range, $0 \mu \mathrm{m}$ representing the edge of the spheroid. C) Average relative fluorescence intensity measured in the mono-culture MCTS shown in panel A for the considered angular range and for the $30 \mathrm{~nm}$ nanoparticles. D. Integrated relative fluorescence intensity profile as a function of the distance from the MCTS edge, for the same experiment as in A-C. The maximum penetration depth is determined as the radial position from the edge corresponding to $95 \%$ of the total integrated relative fluorescence intensity.

nanoparticles. Surprisingly, after $24 \mathrm{~h}$ of perfusion at $10 \mu \mathrm{L} \mathrm{min}{ }^{-1}$, no influence of the nanoparticle size was found, and only the MCTS cellular composition impacted the 
nanoparticle accumulation and penetration. Furthermore, the tailing effect was shorter at $24 \mathrm{~h}$, resulting in a less deep penetration qualitatively ( $c a .50 \mu \mathrm{m})$.
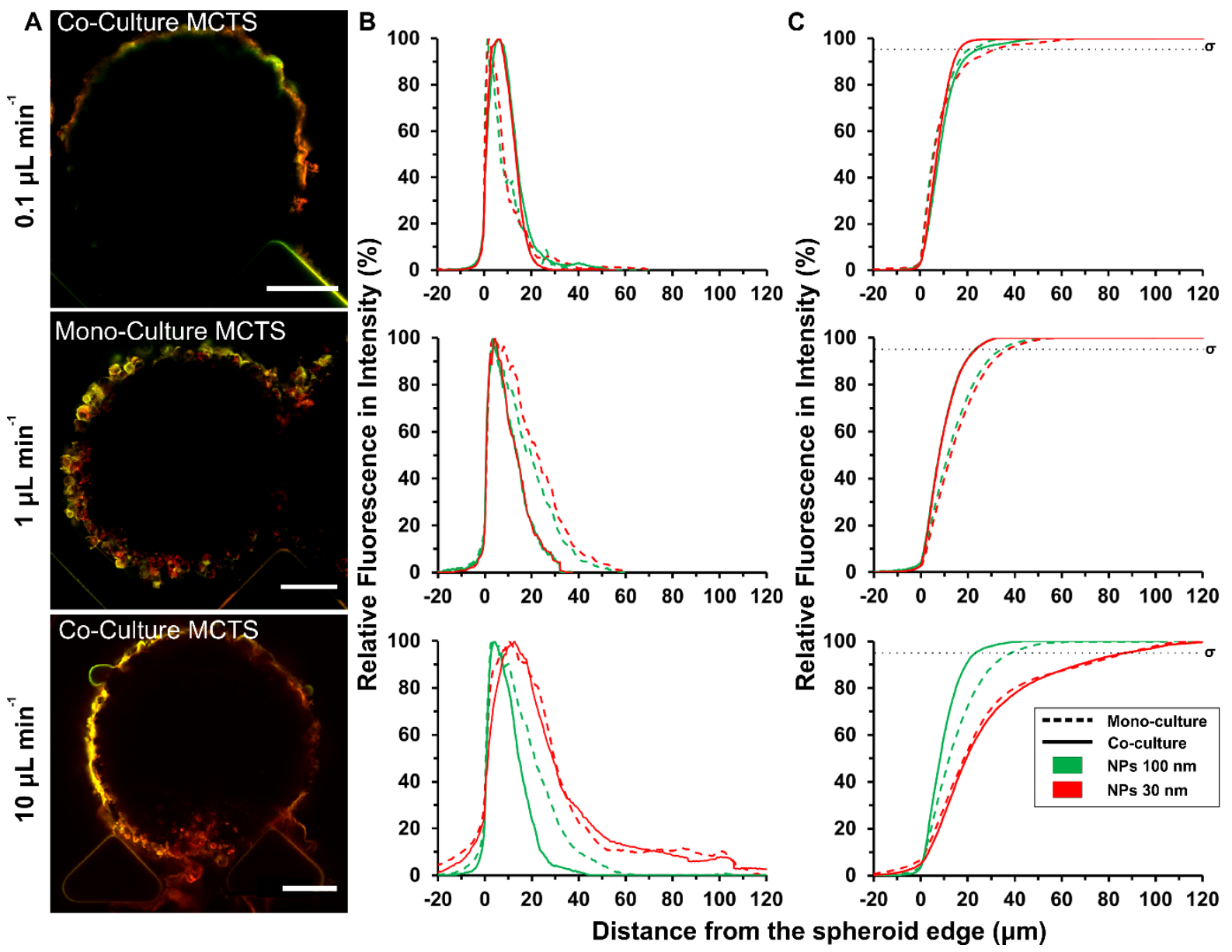

Figure 5-5 Silica nanoparticle penetration in mono- and co-culture MCTS after 48 h continuous perfusion in the microfluidic platform. A) Confocal microscopy picture of MCTS recorded at ca. their half-height in the device, depicting the penetration of the $30 \mathrm{~nm}$ (red) and $100 \mathrm{~nm}$ (green) silica nanoparticles; from top to bottom, at $0.1 \mu \mathrm{L} \mathrm{min}^{-1}$ for a co-culture MCTS, $1 \mu \mathrm{L} \mathrm{min}^{-1}$ for a mono-culture MCTS, and $10 \mu \mathrm{L} \mathrm{min}^{-1}$ for a co-culture MCTS. B) Average relative fluorescence intensity as a function of the distance from the spheroid edge, in mono-culture and co-culture MCTS for the penetration of 30 -

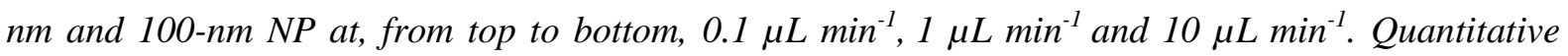
measurements conducted on 6 independent spheroids and averaged. C) Corresponding integrated relative fluorescence intensity profiles as a function of the distance from the MCTS edge.

The maximum of penetration $\sigma$ extracted from the different total penetration profiles (Figure 5-5C) after $24 \mathrm{~h}$ or $48 \mathrm{~h}$ of perfusion at $0.1 \mu \mathrm{L} \mathrm{min}^{-1}$ revealed only a slight penetration, with a large standard deviation, and no significant variation regardless of the nanoparticle size or cellular composition (Figure 5-6). At a 10-time higher flow-rate, the variation of maximum penetration $\sigma$ was still not significant between all different conditions, with $33.3 \pm 5.1 \mu \mathrm{m}(30$ - 
$\mathrm{nm}$ NPs) and $31.2 \pm 5.1 \mu \mathrm{m}(100-\mathrm{nm}$ NPs $)$ in mono-culture MCTS compared to $26.3 \pm 5.0$ and $28.3 \pm 5.6 \mu \mathrm{m}$ in co-culture MCTS, after $48 \mathrm{~h}$. However, a statistical difference could be observed after $48 \mathrm{~h}$ when comparing $\sigma$ between cellular compositions $(\mathrm{p}<0.5)$. When the flowrate was again increased 10-fold, to match the vascular shear stress, the previous situation was reversed, and the nanoparticle size influence on their penetration became dominant in both mono-culture and co-culture, the 30-nm nanoparticles penetrating much deeper $(86.9 .0 \pm 12.2$ $\mu \mathrm{m} v s .91 .6 \pm 19.1 \mu \mathrm{m}$, respectively at $48 \mathrm{~h})$ than the $100-\mathrm{nm}$ ones $(28.3 . \pm 14.2 \mu \mathrm{m} v s .21 .3 \pm$ $6.7 \mu \mathrm{m}$, respectively at $48 \mathrm{~h}$ ).

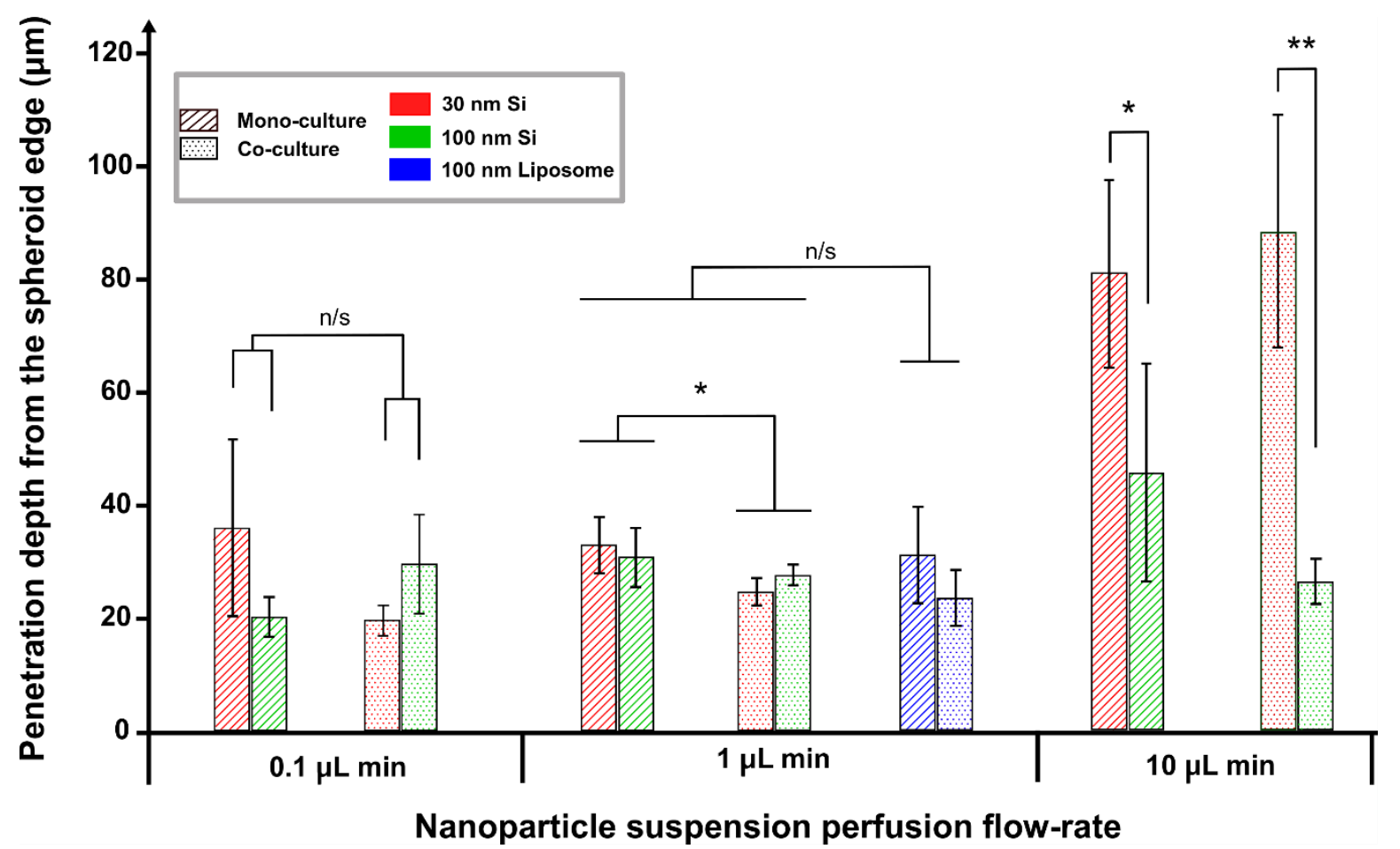

Figure 5-6 Nanoparticle penetration maximum depth for silica nanoparticles of 30 (red) and $100 \mathrm{~nm}$ (green), and $100 \mathrm{~nm}$ liposomes (blue) (all $100 \mu \mathrm{g} \mathrm{mL}^{-1}$ ) in mono- and co-culture MCTS after $48 \mathrm{~h}$ of continuous perfusion at different flow-rates $\left(0.1,1\right.$ and $\left.10 \mu \mathrm{L} \mathrm{min}^{-1}\right)$. The maximum penetration depth was determined as explained in Figure $4 D .(* * p<0.05, * p<0.05, n / s$ not significant, number of spheroids analyzed per condition $n=6)$.

The motion of nanoparticles in suspension is affected by the convective and diffusive properties of the substrate in which they are transported. Inside the microfluidic chamber, this transport is predominantly controlled by the convective flow [3], as also seen in the COMSOL simulation. On the other hand, the high cell density of the MCTS acts as a barrier to the flow, and the transport inside the spheroids becomes diffusion controlled. As a result, independently of the flow-rate selected, the accumulation of the NPs remains essentially on the outer edge of the MCTS. While the data in this study don't present quantitative comparison of the NPs 
accumulation on the MCTS edge, it confirms that, in all conditions, the highest peak of concentration is present at less than $20 \mu \mathrm{m}$ from the edge, which corresponds to only to a few cell layers. These results have previously been reported in similar studies, and interestingly, while in a laminar flow the accumulation on the MCTS surface should be in theory linearly proportional to the flow-rate, Albanese et al. observed only a 2-fold increase of nanoparticle amount at the MCTS edge when increasing the flow-rate by 9-fold [4]. They hypothesized that part of the nanoparticles deposited on the spheroid surface was continuously stripped away by the applied flow.

While in theory the flow-rate does not affect directly the penetration of the NPs inside the tumour spheroid, due to the tissue density and the small pore size in the extra cellular matrix (ECM) [5], the data we present here indicated a much higher penetration at higher flow-rate inside the MCTS. This variation here could be explained by the difference in accumulation level on the MCTS. The diffusion profile is independent of the flow-rate but, following Fick's law of diffusion, is affected by the concentration gradient between two regions with different concentration [6]. As a result of the increase in flow-rate, the concentration gradient between the MCTS edge and its core was greatly affected. The NPs therefore diffused more efficiently inside the spheroids at a higher flow-rate. This conclusion is reinforced by the variation in penetration profile in the different conditions between $24 \mathrm{~h}$ and $48 \mathrm{~h}$. Indeed, while both profiles presented comparable behavior on the outer edge of the MCTS, a low concentration of nanoparticles has diffused much deeper toward the MCTS core after $48 \mathrm{~h}$, increasing with the flow-rate used (Figure 5-5B and 5-S2).

The second major property which affects the diffusion of the NPs, is the density of the MCTS. As previously reported, the presence of stroma inside the tumour can increase significantly the tissue density [7, 8]. Here, the presence of fibroblasts inside the co-culture MCTS affected the penetration of the NPs, which indicates an increase in density of the tissue. Because of the impact of the concentration gradient on the diffusion rate, the impact after $48 \mathrm{~h}$ was only apparent at high flow-rate. Additionally, fibroblasts in a tumour microenvironment are known to secrete increased amount of ECM [9, 10]. This ECM seems to be found predominantly in normoxic region, here at the spheroid edge, locally increasing the density $[11,12]$. At the highest flow-rate we observed a secretion of a transparent dense matter, deposited at the bottom the microfluidic chamber, near the MCTS (Figure 5-S5). When performing the same perfusion experiment without nanoparticles at $10 \mu \mathrm{L} \min ^{-1}$, no such 
secretion was observed. We hypothesized it to be ECM secreted from the MCTS as a result of a combination of high flow-rate shear stress and nanoparticle exposure.

Finally, the diffusion rate of the NPs is affected by their physical properties. This study indicated, as expected, that small NPs $(30 \mathrm{~nm})$ diffused deeper inside the MCTS than the larger $(100 \mathrm{~nm})$ ones. Previous study have observed similar variations and, on average, a threshold in size was observed, approximately at $100 \mathrm{~nm}$ in size, above which the penetration of the NPs became significantly reduced, depending on other nanoparticle properties [1, 4, 13-15]. These additional properties include material, shape, charge, and coating. The silica nanoparticles used in this study were negatively charged, which has shown to improve their penetration, as they are not hindered by electrostatic interactions which would otherwise bind them to the cell surface [16].

Interestingly, in a study of NPs accumulation in in vitro tumour spheroids and in vivo mouse tumours by Mikhail et al., the authors divided the tumour into three different regions in respect to distance to blood vessels: peripheric proliferative, intermediate hypoxic and necrotic, concentric in the spheroid, presenting different cell densities. [17] Results indicated that in vivo, for NPs of $55 \mathrm{~nm}$ in size, a real distinction could be made in term of accumulation, decreasing from the proliferative toward the core. In our study, at the highest flow-rate $(10 \mu \mathrm{L}$ $\left.\mathrm{min}^{-1}\right)$, the distribution profile of the small nanoparticles $(30 \mathrm{~nm})$ changed after reaching $c a .50$ $\mu \mathrm{m}$ in depth, after which the concentration decreased much more slowly. This change in profile seems to be consistent with a change in density in the MCTS, possibly caused by the variation in phenotype from proliferative to hypoxic, where the density is lower [18].

\subsubsection{MCTS exposure to fluorescently-labeled liposomes.}

As a next step, liposomes were tested under the same conditions. Liposomes represent a more relevant model of nanomedicines, and they are currently found on the market, e.g., in the form of Doxil ${ }^{\circledR}$ (doxorubicin-loaded liposomes) or undergoing clinical trials $[19,20]$. Liposomes are soft, in contrast to the hard silica nanoparticles, which allows here examining the still debated influence of this nanoparticle property on their delivery to and penetration in MCTS [21]. Liposomes were prepared using the film-extrusion method from a combination of DPPC (dipalmitoyl phospotidylcholine), DSPE-PEG (PEG2000 - distearoylphosphosphatidylethanolamine polyethylene glycol), and cholesterol, to which a small amount $(0.02 \% \mathrm{~mol})$ of a rhodamine-labeled phospholipid (DHPE-Rhod or dihexadecanoyl-sn-glycero-3- 
phosphoethanolamine-Rhodamine) was added to easily track their penetration in the MCTS. A suspension of 100-nm sized liposomes in serum-free medium and at the same concentration as before $\left(100 \mu \mathrm{g} \mathrm{mL}^{-1}\right)$ was perfused in the microfluidic devices after capture of three MCTS, either mono- or co-culture, for $48 \mathrm{~h}$ (Figure 5-7A). This series of experiments was only conducted using a flow-rate of $1 \mu \mathrm{L} \mathrm{min}{ }^{-1}$, at which a significant impact of the MCTS composition was found on the nanoparticle distribution and delivery, to examine if the same effect would be observed with more clinically relevant nanoparticles.

The comparison between the nanoparticle distribution profiles for the mono- and coculture MCTS (Figure 5-7B) reveals deeper penetration for the less dense and stroma-free MCTS, and in general a similar trend as for the silica nanoparticles of the same size at the same flow-rate (Figure 5-5B). The quantitative analysis of the liposome penetration (Figure 5-6C; Figure 5-7 \& Table 5-S1) confirms these observations: the maximum penetration $\sigma$ after $48 \mathrm{~h}$ was higher in co-culture $(30.5 \pm 9.6 \mu \mathrm{m})$ that in their mono-culture MCTS counterparts $(23.6$ $\pm 5.2 \mu \mathrm{m}$ ), although this difference was not statistically significant ( $>0.5$ ). However, these distribution profiles also show that liposomes could penetrate deeper in the MCTS, as indicated by the longer and deeper tail in the tissue, which would suggest that the NPs diffused more efficiently than the silica nanoparticles.
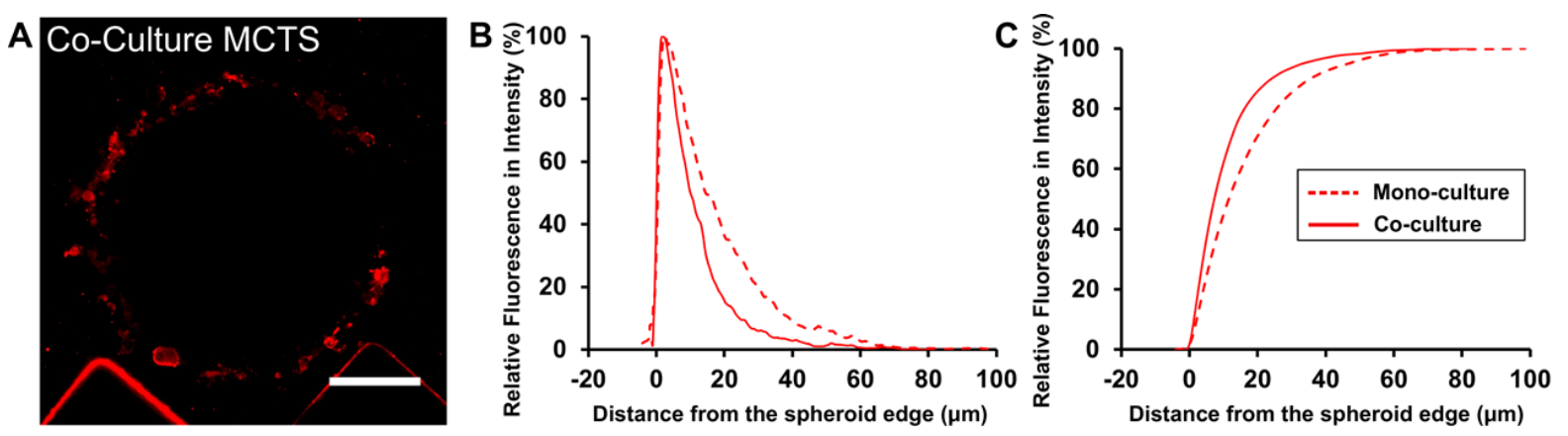

Figure 5-7 Liposome penetration in MCTS after continuous perfusion in the microfluidic device at 1 $\mu L$ min $^{-1}$ for 48 h. A) Confocal microscopy picture of a co-culture MCTS after 48 h perfusion of a rhodamine-labelled liposome solution $\left(100 \mu \mathrm{g} \mathrm{mL}^{-1}\right)$. B) Average relative fluorescence intensity as a function of the distance from the spheroid edge in mono- and co-culture MCTS. C) Corresponding integrated relative fluorescence intensity profiles as a function of the distance from the MCTS edge.

The maximum penetration was very similar for the 100 -nm silica nanoparticles and the liposomes for the co-culture MCTS $(28.3 \pm 5.6 \mu \mathrm{m}$ vs. $30.5 \pm 9.6 \mu \mathrm{m}$, respectively, for the liposomes and the silica nanoparticles) but the liposome showed more superficial accumulation in the monoculture $(31.2 \pm 5.1 \mu \mathrm{m}$ vs. $23.6 \pm 5.2 \mu \mathrm{m}$ respectively, for the liposomes and the 
silica nanoparticles). Noteworthy, no consensus has been reported in the literature on the impact of the nanoparticle stiffness on their delivery in MCTS models [22, 23]. Still, here, liposomes were PEGylated, which introduces another confounding factor [24] in this penetration study compared to the silica nanoparticles of the same size, and their zeta potential was also different (zeta potential $=-2.1 \pm 0.65 \mathrm{mV}$ ) $[13,25]$. Interestingly, the liposome specific composition employed here (DPPC:Chol:DSPE-PEG2000) resembles that used in previous work by Kostarelos et al. on mono-culture prostate cancer MCTS under static incubation (DMPC:Chol:DOPE-PEG2000) [26]; their study revealed poor interactions of those neutral (zeta potential $=4.6 \pm 0.4 \mathrm{mV}$ ) and sterically stabilized liposomes with prostate tumour models due to the presence of the PEG2000 coating, which is in good agreement with the results of the present study.

\subsection{MCTS VIABILITY AFTER NANOPARTICLE EXPOSURE}

Although previous studies have indicated a minimal toxicity of nonporous silica nanoparticles in in vivo studies, [1] prolonged exposure of the MCTS to a continuous flow of nanoparticles can possibly compromise their viability, due to strong local accumulation at the MCTS edge or to the high shear stress they are exposed to. Therefore, as a next step, the viability of the MCTS was evaluated for all tested conditions (mono- and co-culture MCTS, and all three different flow-rate values), the two nanoparticle compositions being jointly perfused in the device as in the penetration experiments. In a first instance, a quantitative assay (CellTiter Glo ${ }^{\circledR}$ 3D) was attempted, in which MCTS were lysed individually and their metabolic activity measured; however, the limited number of cells per MCTS ( $c a$. 10,000 cells per MCTS) gave a low signal-to-noise ratio, which precluded proper cell viability evaluation. Next, standard fluorescent markers were employed to ascertain the viability (Calcein) and death (ethidium homodimer 1 (EthD-1)) of the cells in the MCTS (Figure 5-8A). Since these two stains respectively overlap with the fluorescence of the 100-nm and 30-nm silica nanoparticles, this viability assessment was conducted using non-fluorescent silica nanoparticles, exhibiting the same characteristics as before (30 nm and $100 \mathrm{~nm}$, and carboxy-terminated) and perfused at the same three flow-rate values in serum-free medium at a concentration each of $100 \mu \mathrm{g} \mathrm{mL}$ 1. After $48 \mathrm{~h}$, cell viability was examined in the spheroids (six spheroids analyzed over three separate experiments) by fluorescence microscopy (Figure 5-8A and Figure 5-S6). As a control, MCTS introduced in the device and exposed to a nanoparticle-free serum-free medium perfused at highest flow-rate of $10 \mu \mathrm{L} \mathrm{min}^{-1}$ flow-rate were considered. 
First, in these control experiments, after $48 \mathrm{~h}$ perfusion, both mono- and co-culture MCTS presented a high viability rate $(86.8 \pm 5.9 \%$ and $88.2 \pm 11.8 \%$, respectively), and only a few EthD-1 positive cells were found primarily at the spheroid surface (Figure 5-S6A), indicating that the shear stress the MCTS were exposed to did not impact their overall viability.
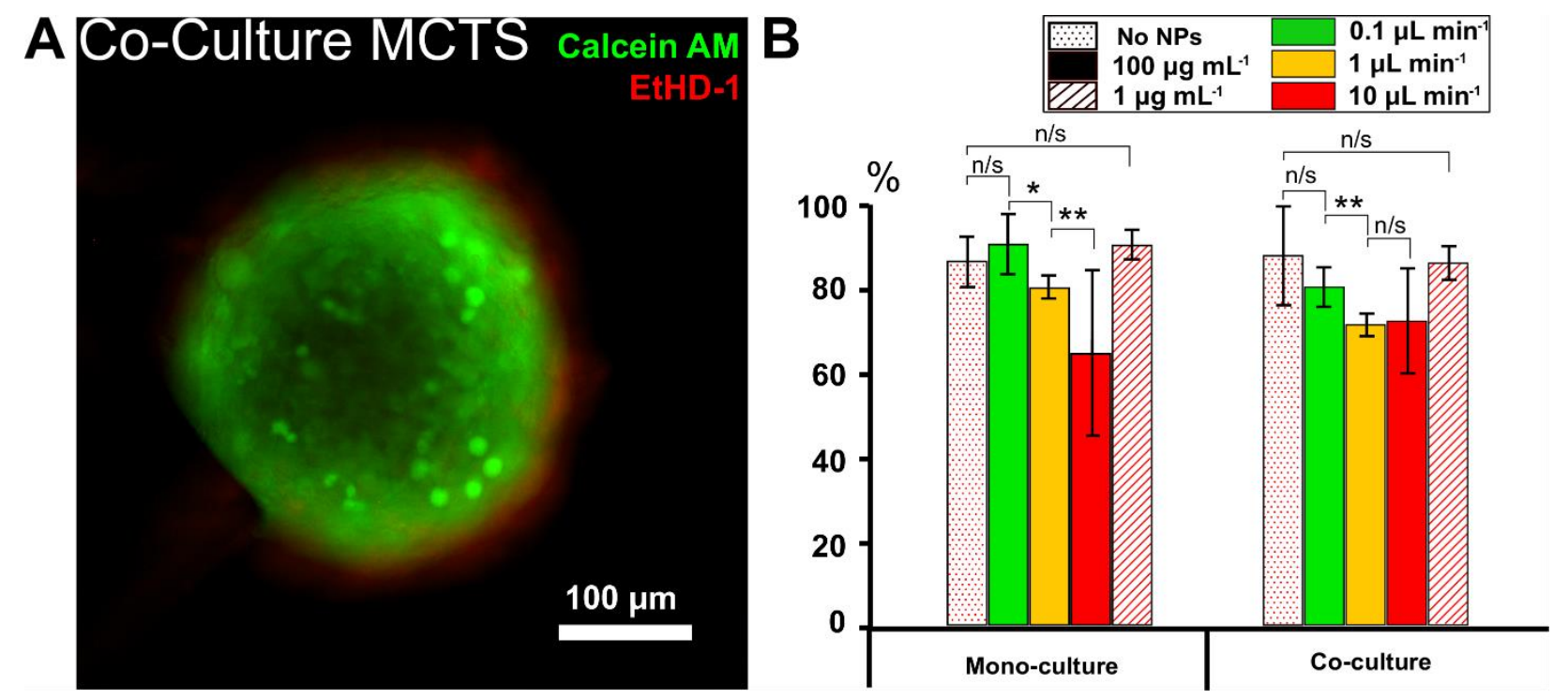

Figure 5-8 MCTS viability in the microfluidic device after continuous exposure to a flow of non-fluorescent silica nanoparticle solution for $48 \mathrm{~h}$. A) Fluorescence microscopy picture of a co-culture MCTS after perfusion at $1 \mu \mathrm{L} \mathrm{min} \mathrm{m}^{-1}$ of 30-nm and 100-nm silica nanoparticles, each at a concentration of $100 \mu \mathrm{g} \mathrm{mL} \mathrm{L}^{-1}$, stained with Calcein (green, live cells) and EthD-1 (red, dead cells); B) Quantitative MCTS viability analysis for both mono- and co-culture MCTS after continuous perfusion $\left(0.1,1\right.$ or $\left.10 \mu \mathrm{L} \mathrm{min}^{-1}\right)$ with $\left(100 \mu \mathrm{g} \mathrm{mL} \mathrm{L}^{-1}\right.$ for all three flow-rates and $1 \mu \mathrm{g} \mathrm{mL} \mathrm{L}^{-1}$ for only $10 \mu \mathrm{L} \mathrm{min}^{-1}$ ) or without nanoparticles (negative control at $10 \mu \mathrm{L}$ $\left.\min ^{-1}\right)$. $(* p<0.5, * * p<0.1, n /$ s not significant, number of MCTS analyzed per condition $n=6)$.

After perfusion of the nanoparticles for $48 \mathrm{~h}$ at a low flow-rate $\left(0.1 \mu \mathrm{L} \mathrm{min}{ }^{-1}\right)$, a few cells (mono-culture MCTS) or a thin layer of cells (co-culture MCTS) facing directly the flow were found dead at the MCTS surface (Figure 5-S6). Yet, the overall MCTS viability did not change significantly $(90.8 \pm 7.1 \%$ and $80.7 \pm 4.6 \%$ for mono- and co-culture MCTS, respectively). When the flow-rate was set to $1 \mu \mathrm{L} \mathrm{min}{ }^{-1}$, a thicker red fluorescent ring was found at the MCTS periphery, the thickness of this ring being $21.3 \pm 4.6 \mu \mathrm{m}$ for the mono- and $23.1 \pm 3.2 \mu \mathrm{m}$ for the co-culture MCTS, which typically corresponds to the a few cell layers. In turn, the MCTS viability was found lower for each MCTS type $(80.5 \pm 2.8 \%$ and $71.8 \pm 2.6$ $\%$ for mono- and co-culture MCTS, respectively). When further increasing the flow-rate to 10 $\mu \mathrm{L} \min ^{-1}$, the ring diameter did not vary significantly $(23.0 \pm 4.5 \mu \mathrm{m}$ for the mono-culture and $25.3 \pm 4.5 \mu \mathrm{m}$ for the co-culture). However, the quantitative assay indicated a great variation between samples, with in average a decrease in viability in the mono-culture MCTS (65.0 \pm 
$19.7 \%)$, but a more constant viability in the co-culture MCTS $(72.6 \pm 12.4 \%)$. Interestingly the higher flow-rate did not impact the viability of the co-culture as much as the mono-culture MCTS. We previously mentioned that according to literature, fibroblasts secretion of ECM can be stimulated in the tumour microenvironment [2]. Since the viability varied differently between the mono- and the co-culture MCTS when increasing the flow-rate, this suggests that the NPs are not encountering the same substrate, and therefore reinforce the hypothesis of ECM being secreted in the spheroid, increasing the tissue density. This hypothesis is reinforced by the observation of secreted ECM inside the device after NPs exposure for $48 \mathrm{~h}$ at $10 \mu \mathrm{L} \mathrm{min}{ }^{-1}$ in co-culture MCTS, which suggests presence of ECM in the outer region of the MCTS (Figure $5-\mathrm{S} 5)$.

Altogether, and interestingly, for one given cellular composition, an increase in the flowrate resulted in a decrease in cell viability, which can be explained either by the higher shear stress the MCTS were exposed to in presence of the nanoparticles, or the 10-100 times higher local accumulation of the nanoparticles at the MCTS periphery. To ascertain which parameter predominantly affected the viability in the MCTS, a last experiment was conducted by perfusing a nanoparticle suspension with a concentration 100 times lower (i.e., each at $1 \mu \mathrm{g}$ $\mathrm{mL}^{-1}$ ) at the highest flow-rate of $10 \mu \mathrm{L} \mathrm{min}{ }^{-1}$. In theory, after $48 \mathrm{~h}$ of perfusion, the accumulation of nanoparticles on the MCTS should be equivalent in these conditions as when using a $0.1-\mu \mathrm{L} \mathrm{min}{ }^{-1}$ flow-rate and a concentration of $100 \mu \mathrm{g} \mathrm{mL}^{-1}$. As depicted in Figures 5S6C and 8B, the MCTS viability in these last conditions was very similar to that found for the lowest flow-rate and the higher nanoparticle concentration $(90.6 \pm 3.4 \%$ vs. $90.8 \pm 7.1 \%$ and $86.3 \pm 3.9 \%$ vs. $80.7 \pm 4.6 \%$, for mono- and co-culture MCTS, respectively), with only a few

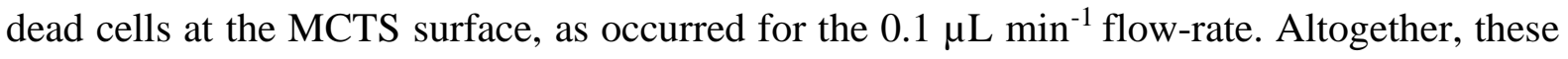
results confirm that the high local accumulation of the nanoparticles at the MCTS surface did compromise the cell viability, and not the high shear stress impact, which is in good agreement with previous studies reporting that nanoparticles at a too high concentration can impair cell viability [3].

\section{Conclusion}

We have successfully developed a microfluidic platform to capture multicellular tumour spheroids, which is compatible with traditional and confocal imaging techniques. We 
tested this model with the perfusion of inert silica and liposomes nanoparticles as a proof-ofconcept to study their penetration inside spheroids of different cellular composition. The presented data indicated that the under interstitial shear stress $(0.01 \mathrm{~Pa})$, the size of the NPs did not affect significantly their penetration inside the tumour. However, when the shear stress was increased $(0.1 \mathrm{~Pa})$, the presence of stroma cells inside the MCTS and major producer of the production of ECM, acted as a barrier to the penetration of the NPs, and the NPs were accumulating at the MCTS periphery. At a vascular shear stress level (1 Pa), the large NPs (100 $\mathrm{nm}$ ) remained at the periphery of the MCTS, while the smaller NPs (30 nm) diffused deeper in the tissue toward the spheroid core. In conclusion, the shear stress plays a role in the penetration of NPs, and therefore controlling this parameter, with for instance the use of ultrasound technology, can help improve drug penetration efficiency.

As mentioned previously, the in vivo tumour is not only composed of different cell types, but is also supplied with nutrients via capillary blood vessels. To reach their target, the NPs must travel in these capillaries, and cross the endothelial barrier first. Although studies have shown that the capillary vessels are leaky in proximity of the tumour, there are no evidence that the tumour itself is exposed to vascular shear stress. As a future research plan, we aim to integrate a capillary vessel network to the tumour spheroid, and assess the NP penetration. The platform presented here will serve as a reference to the impact of the endothelial barrier on the nanomedicine uptake. This system could potentially be used as an early stage platform to study the penetration properties of nanomedicine, especially for those targeting cells presenting a phenotype found deep inside the tissue.

\section{REFERENCES}

1. Ventola, C. L., P T 2017, 42 (12), 742-755.

2. Sun, T.; Zhang, Y. S.; Pang, B.; Hyun, D. C.; Yang, M.; Xia, Y., Angew Chem Int Ed Engl 2014, 53 (46), 12320-64. DOI 10.1002/anie.201403036.

3. Albanese, A.; Tang, P. S.; Chan, W. C., Annu Rev Biomed Eng 2012, 14, 1-16. DOI 10.1146/annurev-bioeng-071811-150124.

4. $\quad$ Frohlich, E., Int J Nanomedicine 2012, 7, 5577-91. DOI 10.2147/IJN.S36111.

5. Wicki, A.; Witzigmann, D.; Balasubramanian, V.; Huwyler, J., J Control Release 2015, 200, 138-57. DOI 10.1016/j.jconrel.2014.12.030.

6. Debbage, P., Curr Pharm Des 2009, $15 \quad$ (2), 153-72. DOI $10.2174 / 138161209787002870$. 
7. Villaverde, G.; Baeza, A., Beilstein J Nanotechnol 2019, 10, 168-181. DOI 10.3762/bjnano.10.16.

8. Imamura, Y.; Mukohara, T.; Shimono, Y.; Funakoshi, Y.; Chayahara, N.; Toyoda, M.; Kiyota, N.; Takao, S.; Kono, S.; Nakatsura, T.; Minami, H., Oncol Rep 2015, 33 (4), 1837-43. DOI 10.3892/or.2015.3767.

9. $\quad$ Nugent, L. J.; Jain, R. K., Cancer Res 1984, 44 (1), 238-44.

10. Tang, L.; Gabrielson, N. P.; Uckun, F. M.; Fan, T. M.; Cheng, J., Mol Pharm 2013, 10 (3), 883-92. DOI 10.1021/mp300684a.

11. Choi, G. W.; Lee, Y. B.; Cho, H. Y., Pharmaceutics 2019, 11 (4). DOI 10.3390/pharmaceutics11040168.

12. Teitelbaum, Z.; Lave, T.; Freijer, J.; Cohen, A. F., Clin Pharmacokinet 2010, 49 (9), 619-32. DOI 10.2165/11533760-000000000-00000.

13. Trujillo-de Santiago, G.; Flores-Garza, B. G.; Tavares-Negrete, J. A.; Lara-Mayorga, I. M.; Gonzalez-Gamboa, I.; Zhang, Y. S.; Rojas-Martinez, A.; Ortiz-Lopez, R.; Alvarez, M. M., Materials (Basel) 2019, 12 (18). DOI 10.3390/ma12182945.

14. Daster, S.; Amatruda, N.; Calabrese, D.; Ivanek, R.; Turrini, E.; Droeser, R. A.; Zajac, P.; Fimognari, C.; Spagnoli, G. C.; Iezzi, G.; Mele, V.; Muraro, M. G., Oncotarget 2017, 8 (1), 1725-1736. DOI 10.18632/oncotarget.13857.

15. Liu, T.; Lin, B.; Qin, J., Lab Chip 2010, 10 (13), 1671-7. DOI 10.1039/c000022a.

16. Sutherland, R. M., Science 1988, 240 (4849), 177-84. DOI 10.1126/science.2451290.

17. Yip, D.; Cho, C. H., Biochem Biophys Res Commun 2013, 433 (3), 327-32. DOI 10.1016/j.bbrc.2013.03.008.

18. Costa, E. C.; Moreira, A. F.; de Melo-Diogo, D.; Gaspar, V. M.; Carvalho, M. P.; Correia, I. J., Biotechnol Adv 2016, 34 (8), 1427-1441. DOI 10.1016/j.biotechadv.2016.11.002. 19. Alkasalias, T.; Moyano-Galceran, L.; Arsenian-Henriksson, M.; Lehti, K., Int J Mol Sci 2018, 19 (5). DOI 10.3390/ijms 19051532.

20. Nunes, A. S.; Barros, A. S.; Costa, E. C.; Moreira, A. F.; Correia, I. J., Biotechnol Bioeng 2019, 116 (1), 206-226. DOI 10.1002/bit.26845.

21. Priwitaningrum, D. L.; Blonde, J. G.; Sridhar, A.; van Baarlen, J.; Hennink, W. E.; Storm, G.; Le Gac, S.; Prakash, J., J Control Release 2016, 244 (Pt B), 257-268. DOI 10.1016/j.jconrel.2016.09.004.

22. He, Z.; Ranganathan, N.; Li, P., Nanotechnology 2018, 29 (49), 492001. DOI 10.1088/1361-6528/aae18a.

23. Lu, H. X.; Stenzel, M. H., Small 2018, 14 (13). DOI ARTN 1702858

10.1002/smll.201702858.

24. Albanese, A.; Lam, A. K.; Sykes, E. A.; Rocheleau, J. V.; Chan, W. C., Nat Commun 2013, 4, 2718. DOI 10.1038/ncomms3718.

25. Huang, K.; Boerhan, R.; Liu, C.; Jiang, G., Mol Pharm 2017, 14 (12), 4618-4627. DOI 10.1021/acs.molpharmaceut.7b00726.

26. Kostarelos, K.; Emfietzoglou, D.; Papakostas, A.; Yang, W. H.; Ballangrud, A.; Sgouros, G., Int J Cancer 2004, 112 (4), 713-21. DOI 10.1002/ijc.20457.

27. Zhang, Y. R.; Lin, R.; Li, H. J.; He, W. L.; Du, J. Z.; Wang, J., Wiley Interdiscip Rev Nanomed Nanobiotechnol 2019, 11 (1), e1519. DOI 10.1002/wnan.1519.

28. Su, Y. L.; Hu, S. H., Pharmaceutics 2018, 10 (4). DOI 10.3390/pharmaceutics10040193.

29. Zhuang, J.; Zhang, J.; Wu, M.; Zhang, Y., Adv Sci (Weinh) 2019, 6 (22), 1901462. DOI 10.1002/advs.201901462.

30. Huang, Q.; Hu, X.; He, W.; Zhao, Y.; Hao, S.; Wu, Q.; Li, S.; Zhang, S.; Shi, M., Am J Cancer Res 2018, 8 (5), 763-777. 


\section{APPENDiX}

\section{MATLAB analysis of NPs penetration.}

The delivery depth of the nanoparticles was analyzed and quantified using an in-house written MATLAB code, examining the nanoparticle penetration from the MCTS edge toward its core in a cross-section image recorded at approximately the half-height of the tissues. Since the MCTS were not always perfectly spherical, this cross-section was not always circular. Therefore, as first step, a circle was drawn around the MCTS cross-section tissue, close to its edge (Figure 5-S1A). The coordinates of the center of this circle were next determined. A series of 130 points was regularly spaced on this circle, within the $120^{\circ}$-wide considered angular range (Figure 5-S1B). Along all these lines, fluorescence intensity profiles were measured and plotted (Figure 5-S1C). The apparent misalignment between these individual profiles is due caused by the non-circularity of the MCTS cross-section. This misalignment was corrected using the position of the spheroid edge for each line (Figures 5-S1D \& 5-S1E). Next, the penetration profiles were plotted using polar coordinates to possibly evaluate the impact of the angle on the nanoparticle penetration depth (Figure 5-S1F). An average nanoparticle penetration profile was obtained for each spheroid condition using the individual 130 plots (Figure 5-S1G), and plotted as relative fluorescence intensity as a function of the distance from the edge of the MCTS. For this, data were normalized to the maximum fluorescence intensity found at the MCTS edge. The $\mathrm{x}$ axis was converted from pixel values to micrometers by measuring the scalebar on the image. Finally, the cumulative fluorescence intensity was plotted as a function of the distance from the edge of the MCTS by integrating the fluorescence intensity in the nanoparticle penetration profile as a function of the distance from the edge of the MCTS (Figure 5-S1H). Using this last graph, the maximum penetration depth $\sigma$ was determined as the position corresponding to $95 \%$ of cumulative fluorescence intensity, as depicted on Figure 5-S1H. 

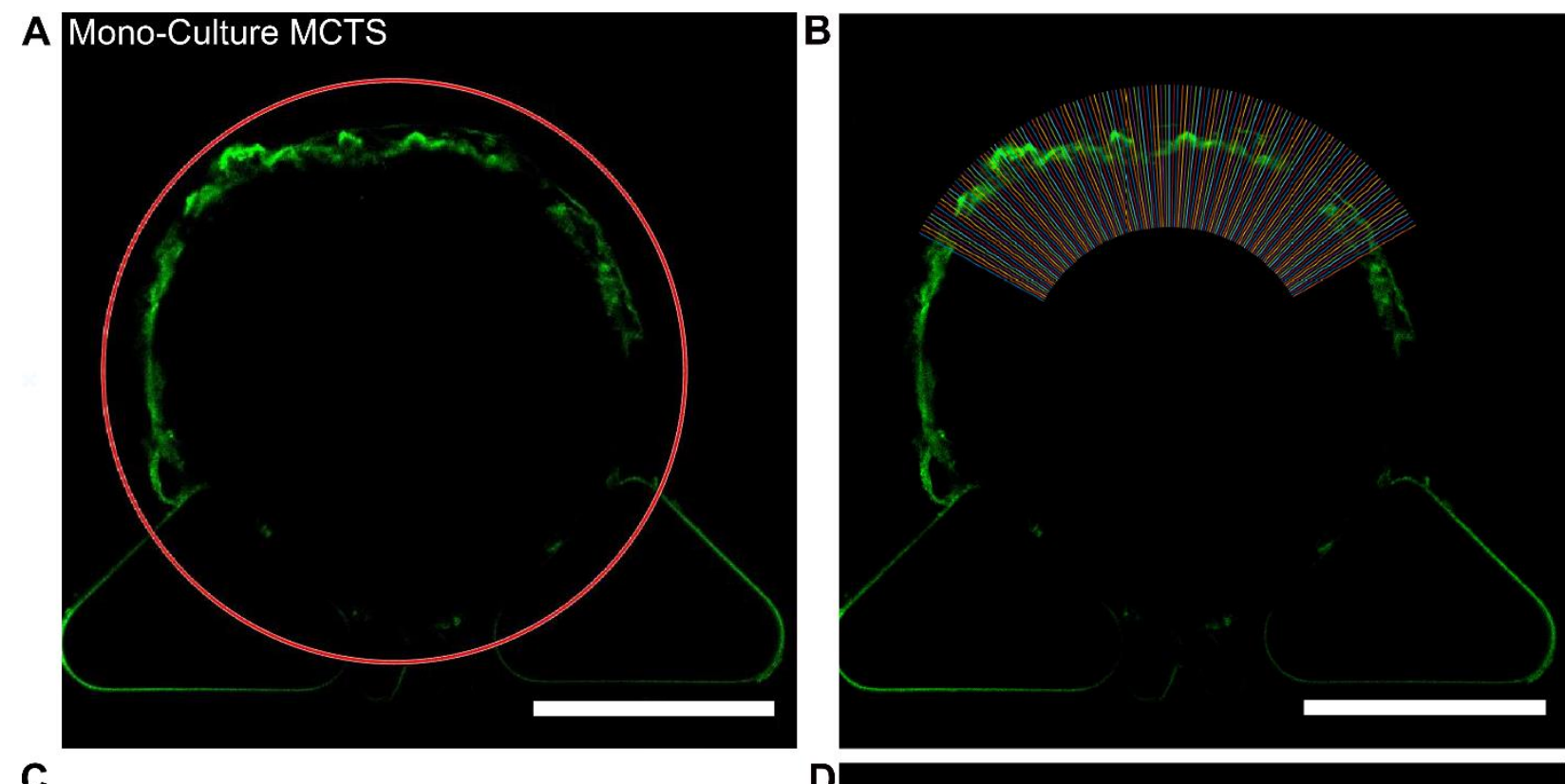

c
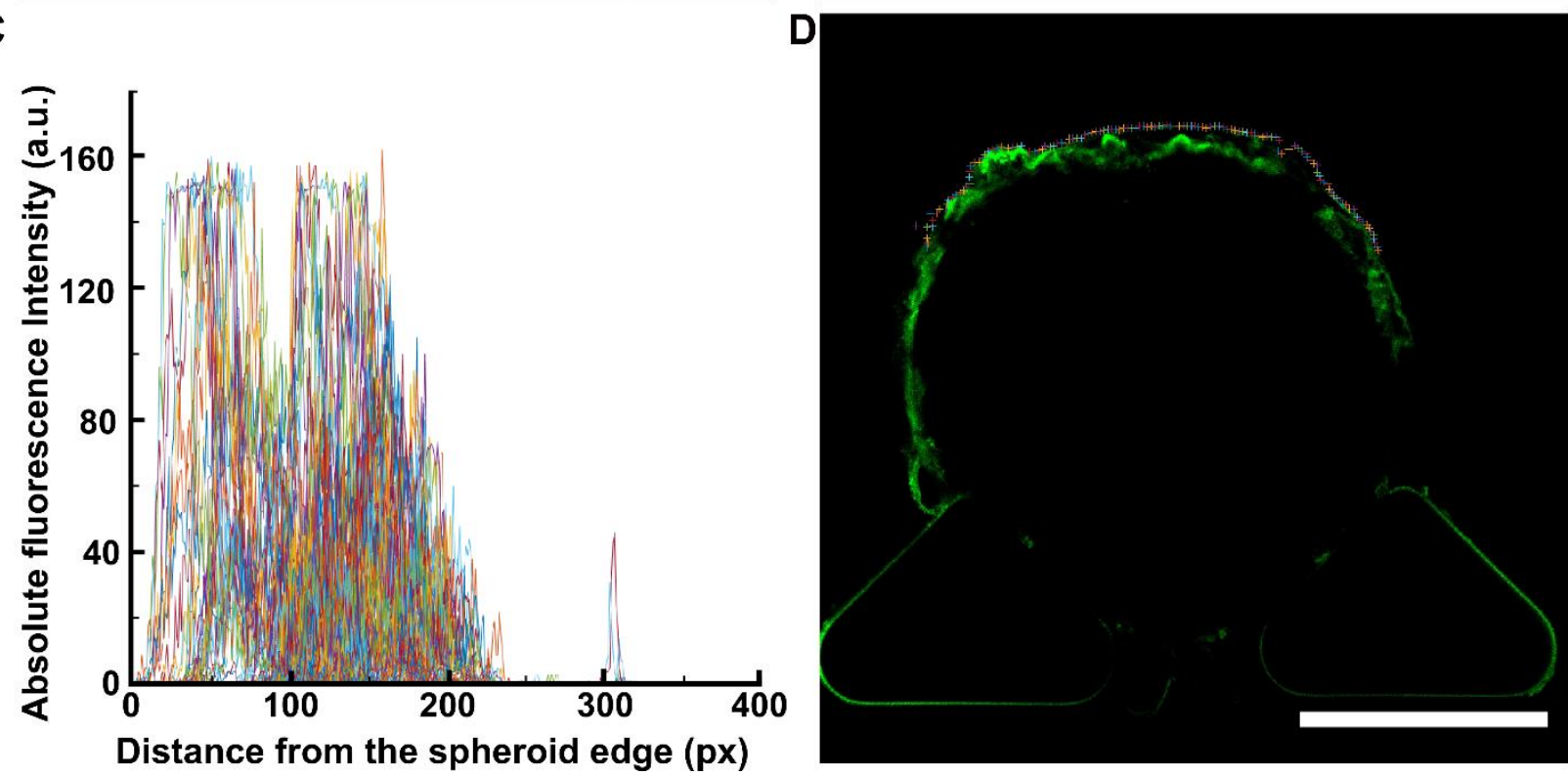

Figure 5-S1. MATLAB analysis of the nanoparticle penetration in MCTS models in the microfluidic system; example of the penetration of $100 \mathrm{~nm}$ green fluorescent nanoparticles in a mono-culture MCTS after $48 \mathrm{~h}$ perfusion at $10 \mu \mathrm{L} \mathrm{min} \mathrm{m}^{-1}$. A) Circle of acquisition drawn around the MCTS considered crosssection. B) Determination of 130 vectors regularly spaced along a $120^{\circ}$-wide angular region. C) Fluorescence intensity profiles measured along each vector as a function of the distance from the MCTS edge (pixels). D) Positions of intersections between spheroid edge and each vector. E) Fluorescence intensity profiles aligned for the 130 considered vectors F) Fluorescence profile plotted in polar 
E

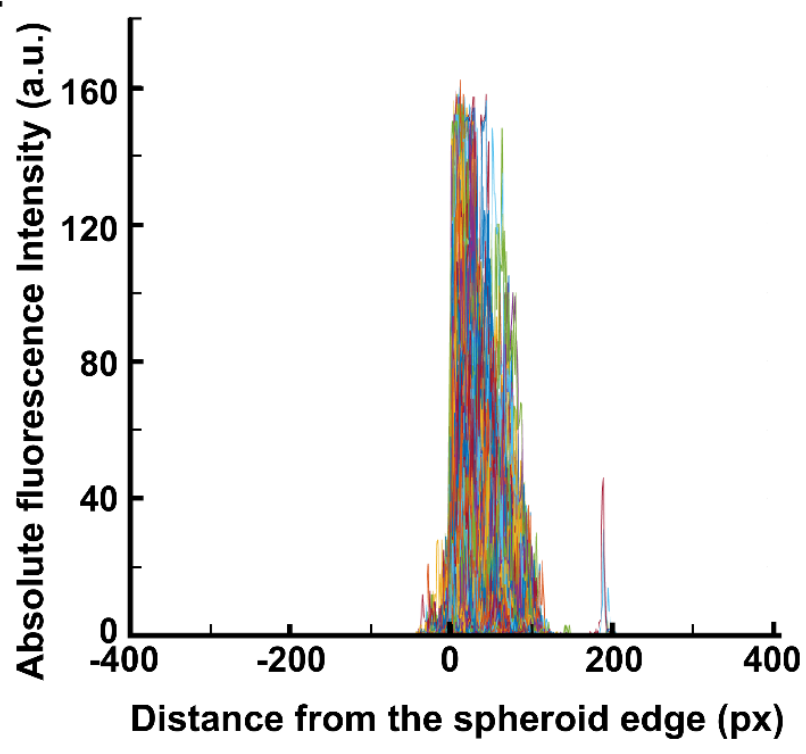

G

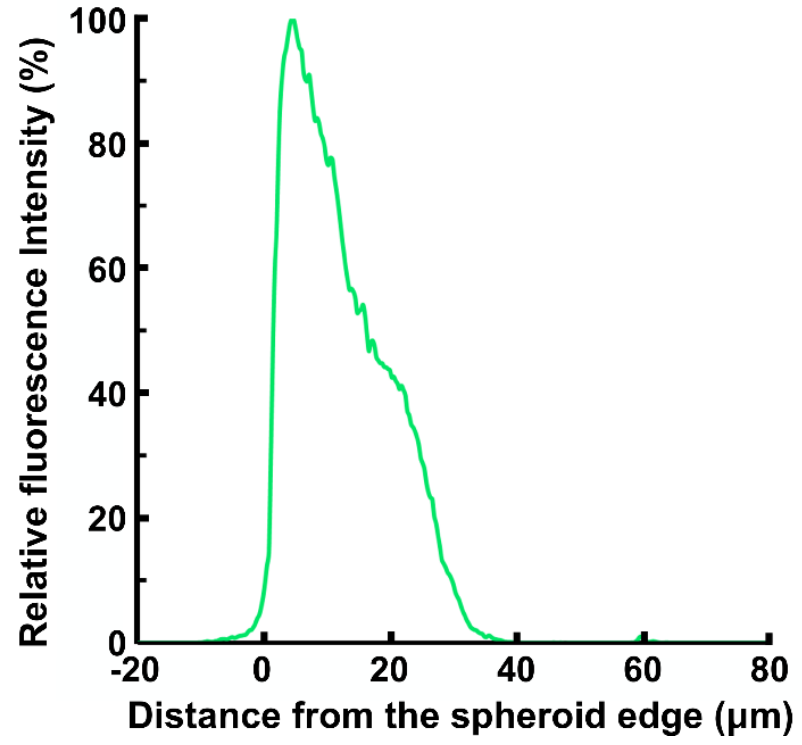

$\mathbf{F}$

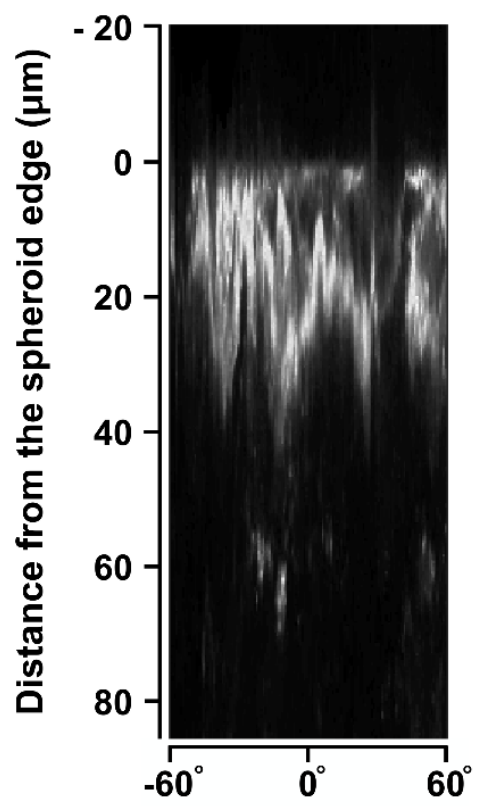

Angle of acquisition

H

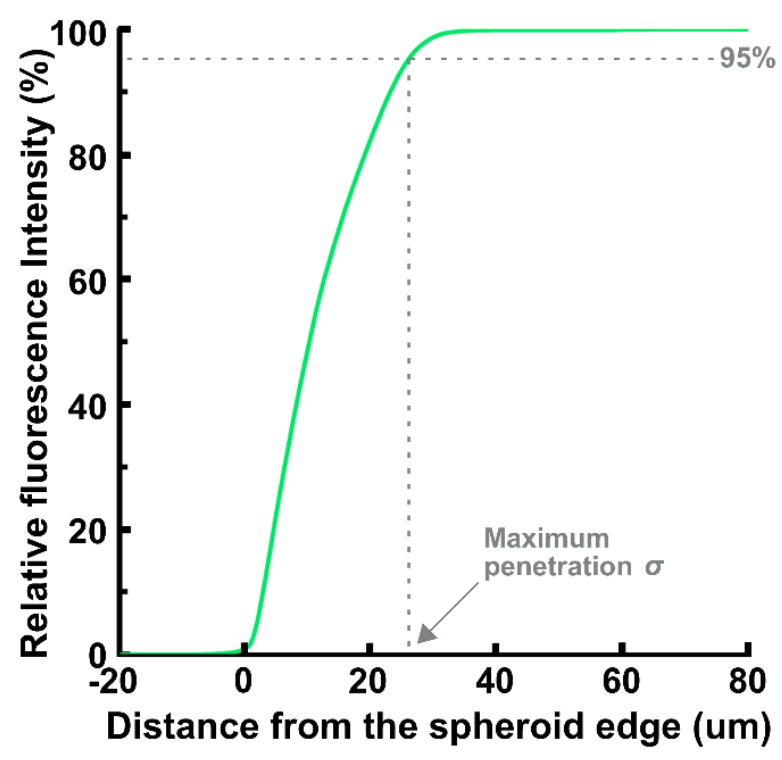

coordinates for the considered angular range; G) Nanoparticle penetration profile plotted as the relative fluorescence intensity as a function of the distance from the edge of the MCTS, and averaged for all 130 vectors; $H$ ) Cumulative fluorescence intensity profile (relative values) as a function of the distance from the edge of the MCTS, corresponding to the penetration profile presented in $(G)$. 

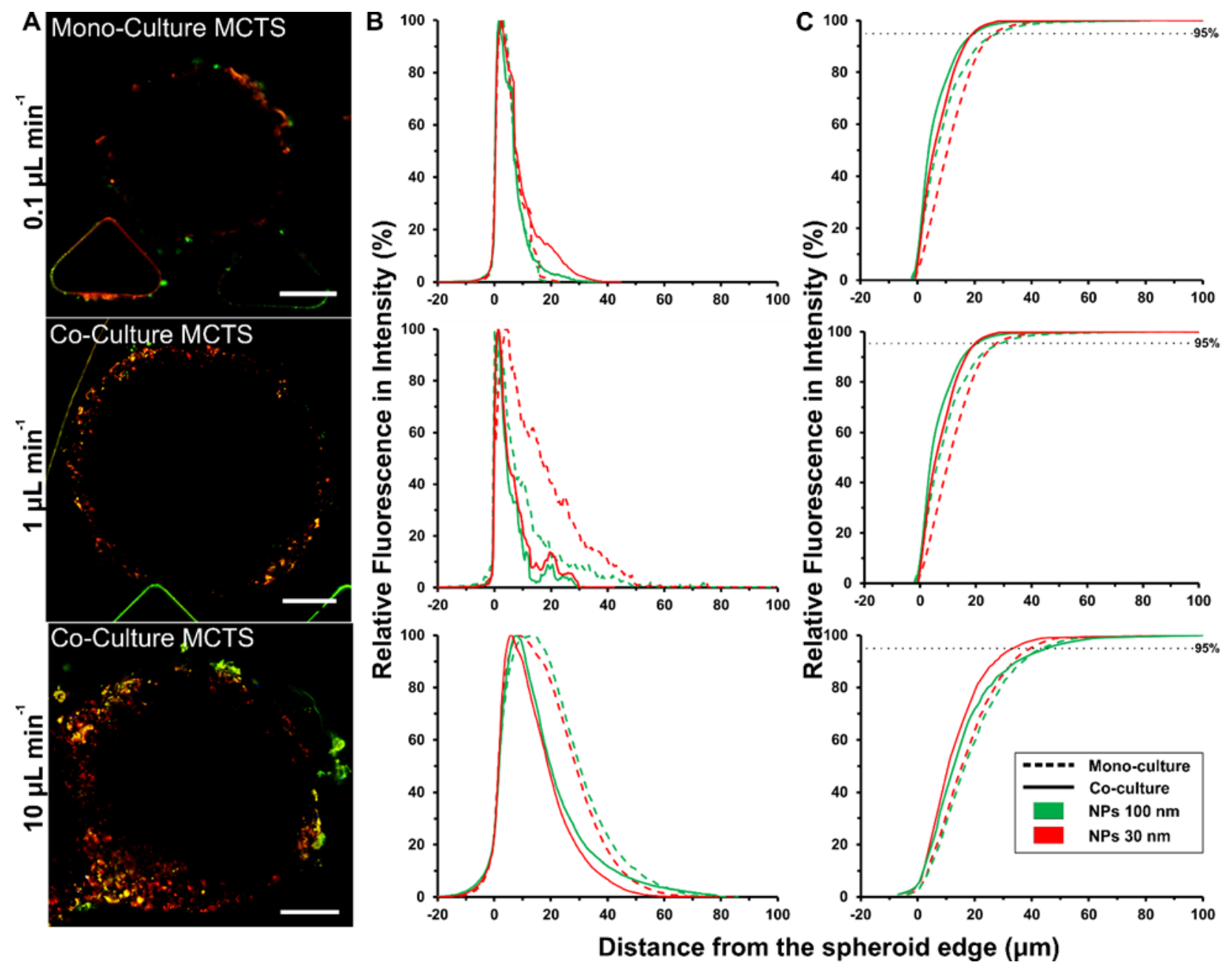

Figure 5-S2. Silica nanoparticle penetration in mono- and co-culture MCTS after $24 \mathrm{~h}$ perfusion in the microfluidic device. A) Confocal microscopy images of the MCTS cross-section at half-height for the penetration of the $30 \mathrm{~nm}$ (red) and 100-nm (green) nanoparticles after perfusion for $24 \mathrm{~h}$ at a flow-rate of $0.1,1,10 \mu \mathrm{L} \mathrm{min}^{-1}$. B) Nanoparticle penetration profile plotted as a relative fluorescence intensity as a function of the distance from the spheroid edge, in mono-culture and co-culture MCTS for the penetration of 30-nm and 100-nm nanoparticles at, from top to bottom, $0.1 \mu \mathrm{L} \mathrm{min}^{-1}, 1 \mu \mathrm{L} \mathrm{min}^{-1}$ and 10 $\mu L \mathrm{~min}^{-1}$. These measurements were conducted on six independent spheroids in 3 independent experiments and averaged to yield these graphs. C) Corresponding cumulative fluorescence intensity as a function of the distance from the MCTS edge for the same experiments as in (B). 


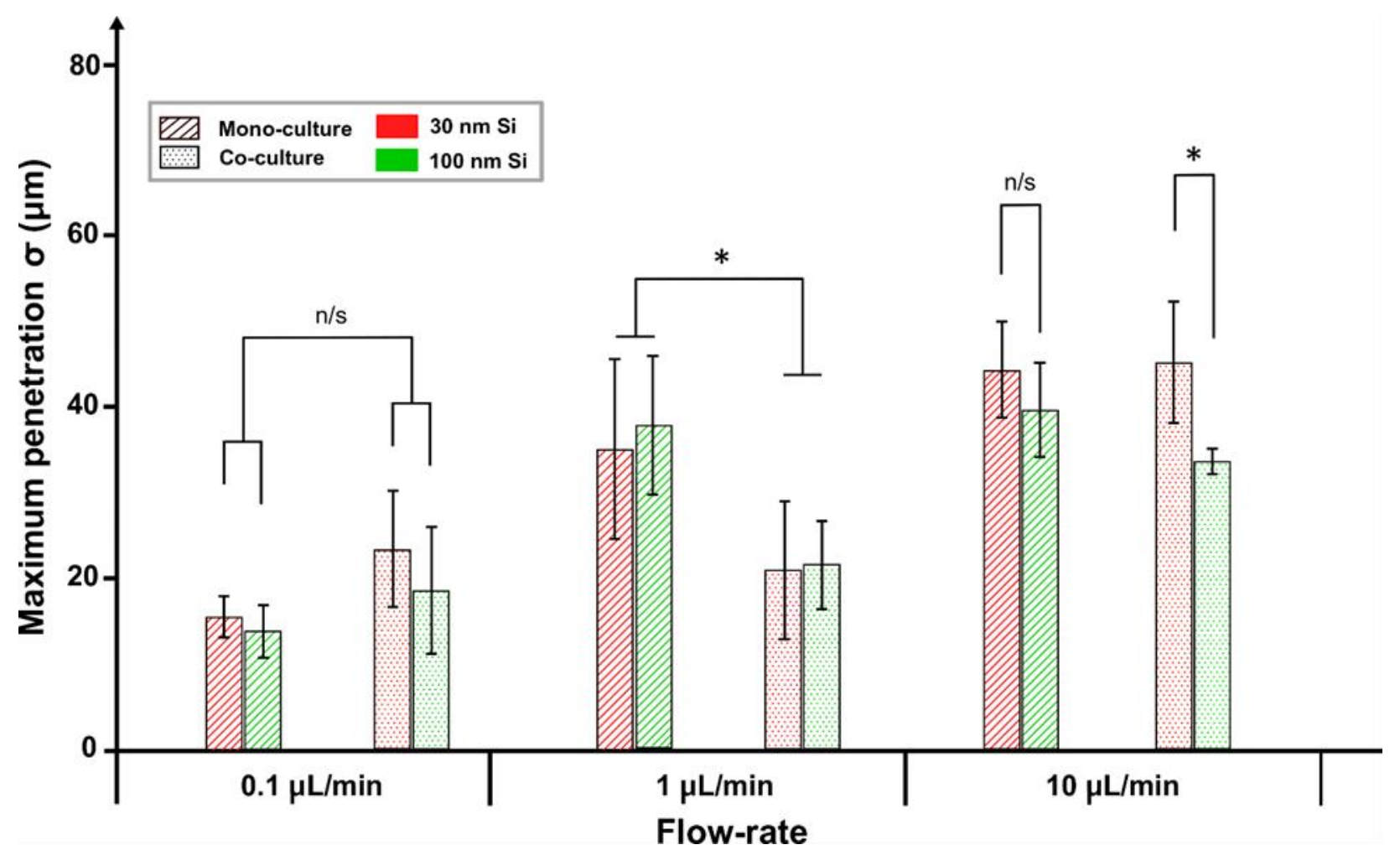

Figure 5-S3. Maximum penetration depth $\sigma$ of silica nanoparticles in mono- and co-culture MCTS after $24 \mathrm{~h}$ of perfusion at different flow-rates (0.1, 1 and $\left.10 \mu \mathrm{L} \mathrm{min}{ }^{-1}\right)$ of 30 (red) and $100 \mathrm{~nm}$ (green) nanoparticles introduced at a concentration of $100 \mu \mathrm{g} \mathrm{mL} L^{-1} \quad(* * p<0.01, * p<0.05, n / s$ not significant $p>0.5, n=6)$. 
Table 5-S1. Summary of the maximum penetration depth $\sigma$ of 30-nm and 100-nm silica nanoparticles after $24 \mathrm{~h}$ and $48 \mathrm{~h}$ of perfusion at different flow-rates $\left(0.1,1,10 \mu \mathrm{L} \mathrm{min}^{-1}\right)$, and of $100 \mathrm{~nm}$ liposomes

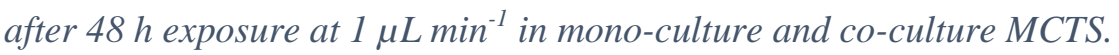

\begin{tabular}{|c|c|c|c|c|c|c|}
\hline & \multirow{2}{*}{\multicolumn{2}{|c|}{$\boldsymbol{\sigma}(\mu \mathrm{m})$}} & \multicolumn{2}{|c|}{$24 h$} & \multicolumn{2}{|c|}{$48 h$} \\
\hline & & & Mono-culture & Co-culture & Mono-culture & Co-culture \\
\hline \multirow{6}{*}{ SiNPs } & \multirow{2}{*}{$0.1 \mu \mathrm{L} / \mathrm{min}$} & $30 \mathrm{~nm}$ & $15.9 \pm 2.4$ & $23.5 \pm 6.8$ & $40.0 \pm 18.7$ & $33.5 \pm 1.5$ \\
\hline & & $100 \mathrm{~nm}$ & $14.0 \pm 3.1$ & $18.7 \pm 7.4$ & $21.3 \pm 3.2$ & $26.4 \pm 5.6$ \\
\hline & \multirow{2}{*}{$1 \mu \mathrm{L} / \min$} & $30 \mathrm{~nm}$ & $35.3 \pm 10.5$ & $21.0 \pm 8.0$ & $33.3 \pm 5.1$ & $26.3 \pm 5.0$ \\
\hline & & $100 \mathrm{~nm}$ & $37.8 \pm 8.1$ & $21.6 \pm 5.2$ & $31.2 \pm 5.1$ & $28.3 \pm 5.6$ \\
\hline & \multirow{2}{*}{$10 \mu \mathrm{L} / \mathrm{min}$} & $30 \mathrm{~nm}$ & $44.4 \pm 5.6$ & $45.3 \pm 7.1$ & $86.9 \pm 12.2$ & $91.6 \pm 19.1$ \\
\hline & & $100 \mathrm{~nm}$ & $39.7 \pm 5.4$ & $33.7 \pm 1.4$ & $28.3 \pm 14.2$ & $21.3 \pm 6.7$ \\
\hline iposome & $1 \mu \mathrm{L} / \mathrm{min}$ & $100 \mathrm{~nm}$ & N/A & $N / A$ & $23.6 \pm 5.2$ & $30.5 \pm 9.6$ \\
\hline
\end{tabular}




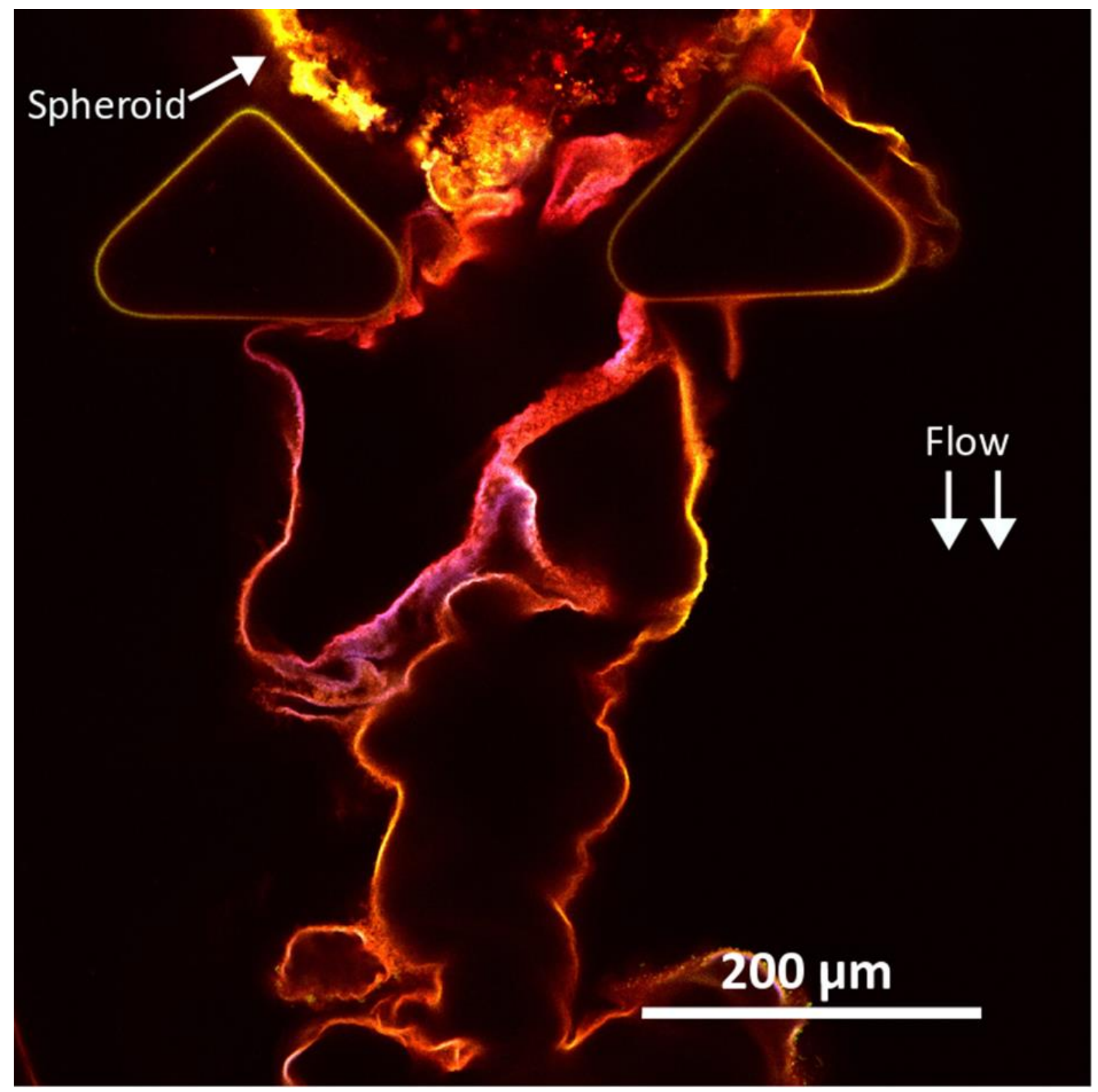

Figure 5-S5. Confocal microscopy picture of a co-culture MCTS (cross-section at half-height) in the microfluidic device, after $48 \mathrm{~h}$ perfusion at $10 \mu \mathrm{L} \mathrm{min}^{-1}$, of a solution of $30 \mathrm{~nm}$ (red) and $100 \mathrm{~nm}$ (green) fluorescent silica nanoparticles, showing the creation of a significant tail presumably of extra-cellular matrix, as a response to the stress induced by the combination of the flux and the nanoparticle solution. 
Mono-Culture MCTS
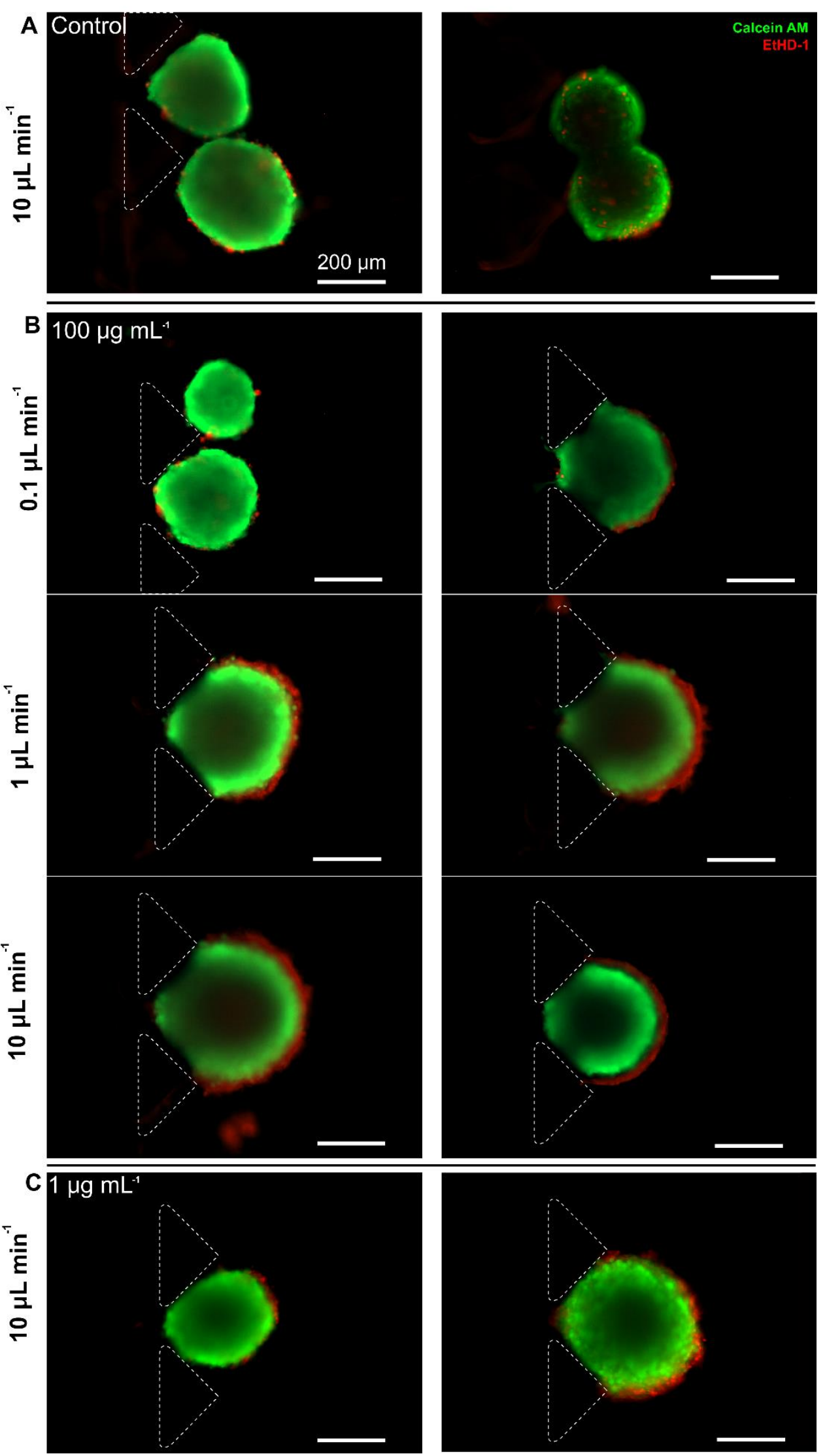
Figure 5-S6. Viability assessment of MCTS in the microfluidic device after exposure for $48 \mathrm{~h}$ to the (non-fluorescent) nanoparticle suspension, using a live/dead assay and the calcein (live, green) and EtHD-1 (dead, red) probes. Microscopy pictures recorded with an EVOS microscope in the device for: A) mono- and co-culture MCTS exposed to a flow-rate of $10 \mu \mathrm{L} \mathrm{min}^{-1}$ with no nanoparticle (control). B) Mono- and Co-culture MCTS exposed to a flow-rate of 0.1, 1, and $10 \mu \mathrm{L} \mathrm{min}{ }^{-1}$ with 30 and $100 \mathrm{~nm}$ silica nanoparticles, each at $100 \mu \mathrm{g} \mathrm{mL} \mathrm{L}^{-1}$. C) Mono- and co-culture MCTS exposed to a flow-rate of 10

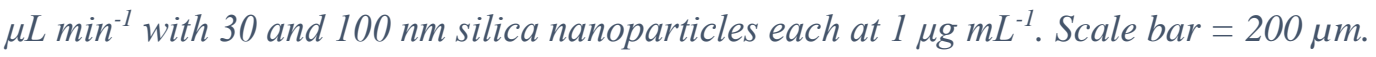




\section{Chapter 6 - Toward Vascularized Breast Cancer on Chip: Approaching the Complex Tumour Micro Environment}

\section{Abstract}

Building on the previous chapters, here we aimed at increasing the complexity of our tumour-on-chip model by combining a vascular system with our tumour spheroid. We designed a microfluidic device, in which a single tumour spheroid could be trapped, and flanked by two large blood vessels. By generating a gradient of growth factors from the tumour site toward those large blood vessels, the process of angiogenesis could be triggered to yield an early neovasculature. In this chapter, we prepared tumour spheroids from human breast cancer cells (MDA MB-231), human mesenchymal stem cells (hMSCs) and Human Umbilical Vein Endothelial Cells (HUVECs). The large blood vessels were prepared using the viscous finger patterning method in collagen I hydrogel, and lumens of $c a .400 \mu \mathrm{m}$ in diameter were reproducibly generated. Blood vessels were next obtained by seeding HUVECs inside the lumens. Angiogenesis was initiated by perfusing a gradient of Vascular Endothelial Growth Factor (VEGF), and was supported by the presence of hMSCs inside the collagen gel in which the lumens were created. Capillary vessels successfully sprouted from the large blood vessels towards the tumour site, and were $c a .500 \mu \mathrm{m}$ in length. 


\section{INTRODUCTION}

The high density of tumours, as well their size and cellular composition, results in the creation of gradients of oxygen and nutrient depletion toward the core of the diseased tissues. To be able to sustain itself, the tumour becomes angiogenic [1]: tumour cells secrete growth factors to recruit endothelial cells to form new blood vessels in the tumour tissue. These endothelial cells migrate from blood vessels surrounding the tumour toward its core. This process called neovascularization consists of the formation of capillary blood vessels through which nutrients and oxygen can be delivered to the rapidly expanding tumours [2]. As such, vascularization is a key element in the development of the tumour microenvironment (TME), and although the multicellular tumour spheroid (MCTS) is a good model of tumour, it does not incorporate this vasculature [3-5].

In vivo bloods vessels can form through two main mechanisms. On one hand, vasculogenesis, which is found in embryos, starts with the differentiation of stem cells into endothelial cells to form the circulatory system. On the other hand, angiogenesis, supports the creation of new blood vessels from existing ones [1]. This second process is triggered by specific proteins, or "pro-angiogenic factors", which are normally secreted by neighboring cells in the need of nutrients and/or oxygen [6]. Blood vessels can be classified in different categories based on their size, from large to small: artery, vein, capillary. The newly formed blood vessels, or so-called neovasculature, are always first capillaries, which normally grow in size together with the surrounding tissue [7]. Additionally, the neovascularization is supported by other cell types, especially stromal cells (i.e., smooth muscle cells and pericytes), which are essential for the formation of stable and leak-proof blood vessels. Due to their slow development (a few hundred micrometers per day), controlling the formation of blood vessels is a great challenge, especially in the field of regenerative medicine [8]. Vascularization is actually the main limiting factor to the development of artificial tissues; currently such tissues do not possess a structured vascular network, and therefore, in the case of transplantation, die out of starvation before the surrounding tissues can provide a vascular network [9, 10]. As a result, vascularization is now being highly investigated to optimize and accelerate these two angiogenesis and neovascularization processes. 
Using microfluidic devices, two main approaches have been developed for the formation of blood vessels. The first one uses a bottom-up strategy, where endothelial cells are injected in cylindrical lumens previously created inside a hydrogel matrix. There, the cells selfassemble into a system resembling the blood vessel conformation [11]. This cylindrical structure can be by introducing a cylindrical wire or rod with a specific diameter in a hydrogel matrix, and extracting it after gelation to leave a lumen with a similar diameter as the wire or rod [12]. Alternatively, the "viscous finger patterning" (VFP), which was developed by Bischel et. al [13] can be used; it uses one of the unique properties of microfluidics, which is the laminar character of the flow. Passive pumping [14], a method by which a flow is driven inside the microfluidic channels by the capillary forces and oriented by a difference of pressure, is used to perfuse medium through a channel pre-filled with an un-polymerized hydrogel. In the process, a lumen is created in this hydrogel, whose dimensions can be controlled by the hydrogel properties, the capillary forces and the pre-gelation time. While the former bottomup approach is powerful to quickly produce blood vessels, it is limited as it can only produce straight lumens, whereas blood vessels naturally grow as arborescence-like structures. Additionally, the minimum size of the lumen is limited by either method to $c a .100 \mu \mathrm{m}$ in diameter, whereas capillary blood vessels are on average $5 \mu \mathrm{m}$ in diameter.

The second approach developed for the formation of blood vessels is inspired by the natural manner blood vessels form: angiogenesis. Neovascularization can be initiated by placing endothelial cells in or against a hydrogel matrix, and stimulated by using right combination of cell types and growth factors [15-17]. Especially, studies have shown that sprouting and migration of endothelial cells could be triggered by (a gradient of) Vascular Endothelial Growth Factor (VEGF). To obtain stable capillary blood vessels however, VEGF has shown to be insufficient, and several studies have been focusing on the impact of additional growth factors (e.g., fibroblast growth factor (FGF), angiopoietins, or platelet derived growth factors (PDGF)) [18, 19]. The main limitations of the angiogenesis-on-chip approach are the speed at which neovascularization occurs (less than one millimeter per day) and the maximum distance across which sprouting occurs (ca. $2 \mathrm{~mm}$ ) [20].

As a next step, and in the view of creating a complete TME, 3D multicellular tumour spheroids must be combined with such a vascular system. Such platform could potentially become a new in vitro cancer model, providing information on the drug uptake from the blood stream, or on the cancer metastasis mechanism. Recently a few studies have reported organon-chip devices combining tumour spheroid and a neovasculature [21, 22]. The relevance of 
the proposed models is however arguable, due to the chosen cellular composition, which combines cell types from different unrelated organs. For instance, Sano et al. successfully developed a vascularized TME model, but only by combining osteoblast (bone cells) spheroids and lung fibroblasts [23].

In this chapter we present a microfluidic device designed to create a vascularized breast tumour model, starting from a single co-culture human MCTS to eventually yield a complex vascularized TME. Here, we adapted our system compared to the previous chapters toward a human model, which is obviously more relevant for the development and testing of drugs. We first engineered a complex vascular system by integrating the two blood vessels formation approaches previously described. First, we used the bottom-up approach and the VFP method, to generate two large blood vessels acting as vein models present near a tumour. Between these two blood vessels, a human co-culture MCTS composed of human breast cancer cells, hMSCs and endothelial cells, was trapped. Next, we triggered angiogenesis from the two blood vessels, after creating a gradient of VEGF, in order to generate a neovascularization toward the spheroid. Finally, integrating stromal cells (i.e., Mesenchymal Stem Cells) in the collagen hydrogel provided additional growth factors, assisting the neovascularization in our microfluidic device.

\section{MATERial AND MethodS}

\subsection{MICROFLUIDIC CHIP FABRICATION}

The microfluidic device contained three main features: 2 lateral channels of 600x600 $\mu \mathrm{m}^{2}$ (cross-section) with a constriction, a central channel of $500 \mu \mathrm{m}$ in width and $600 \mu \mathrm{m}$ in height, and 2 connecting channels of $400 \mu \mathrm{m}$ in width and $200 \mu \mathrm{m}$ in height (Figure 6-1). To ensure the connecting channels would be located at the half height with respect to the side channels, two identical fluidic layers were assembled, each with half of the microfluidic structures in height as depicted in Figure 6-1.

Two negative molds of the microfluidic chip were designed using SolidWorks (Dassault Systèmes, Vélizy-Villacoublay, France), and 3D printed in resin (PIC100, EnvisionTEC, Dearborn, MI, USA) using a 3D printer (Perfactory, EnvisionTEC) with a resolution of $50 \times 50 \mu \mathrm{m}^{2}$, followed by a UV curing step $\left(1 \mathrm{~h}, \lambda=254 \mathrm{~nm}\right.$, Power $\left.{ }_{\text {lamp }}=15 \mathrm{~W}\right)$ and 
a $24 \mathrm{~h}$ baking step at $60^{\circ} \mathrm{C}$ in water. Polydimethylsiloxane (PDMS) was prepared by mixing a pre-polymer and a curing agent in a 10:1 weight ratio (SYLGARD 184, Dow Corning, Midland, MI, USA). After a degassing step in vacuum, the mixture was poured into both molds in such a way that they were completely filled without formation of a meniscus. The PDMS was then cured $\left(60^{\circ} \mathrm{C}, 3 \mathrm{~h}\right)$ and peeled off from each mold, to obtain the two microfluidic layers: a thick "top" layer (ca. $1.9 \mathrm{~mm})$ and a thin "bottom" layer (ca. $0.5 \mathrm{~mm})$. Holes of $1 \mathrm{~mm}$ in diameter for the inlets and $1.5 \mathrm{~mm}$ in diameter for the outlets were punched in the top PDMS layer, using a puncher (Ted Pella, Redding, CA, USA).

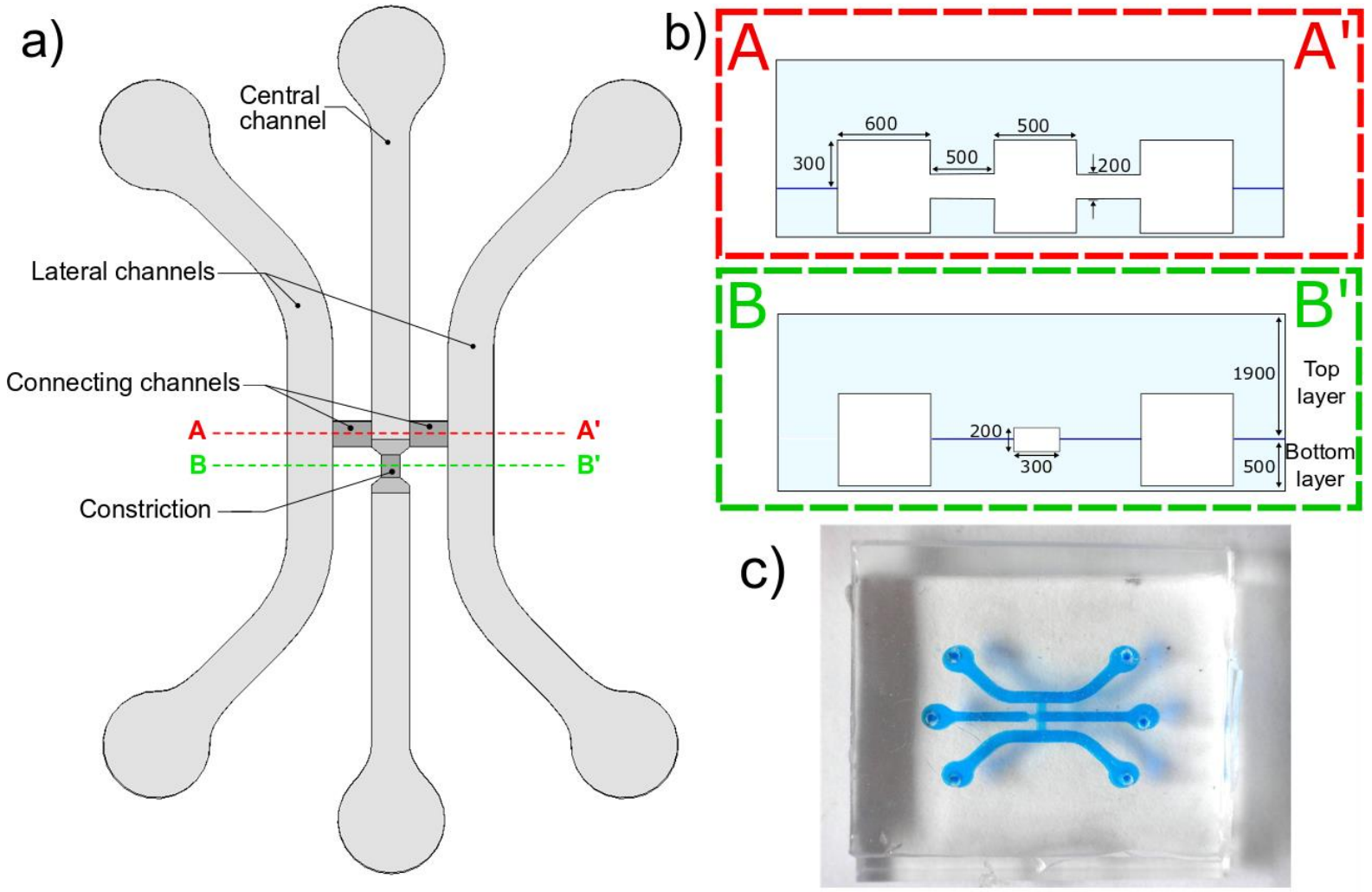

Figure 6-1 Tumor Microenvironment on chip - Microfluidic design. a) Top view of the design of the microfluidic device, which comprises three main sections: two lateral channels, one central channel, and two connecting channels. A constriction is placed in the central channel to trap a single tumor spheroid at the level of the connecting channels. b) Cross sections of the device, indicated as a red dashed line in (a) at the location where the connecting channels are found, and with a green dashed box in (a) at the level of the constriction. The connecting channels and constriction are placed at the mid-height of the channels by aligning and bonding a "bottom layer" and "top layer" with the same fluidic structures. Distances are expressed in micrometres. c) Picture of a microfluidic device in PDMS, after aligning and bonding, and microfluidic channels filled with blue dye for visualisation purposes.

Both layers and a glass coverslip (VWR, Radnor, USA) were exposed to plasma treatment (CUTE plasma cleaner, Femto Science Inc., Gyeonggi-Do, Korea) for $45 \mathrm{~s}$ at 
$5 \times 10^{-1}$ mTorr. The bottom layer was first assembled to the glass coverslip, leaving the fluidic structure exposed. The top layer was next bonded to the bottom layer, while taking care to align the fluidic structures under microscope.

\subsection{Cell Culture}

Human Umbilical Vein Endothelial Cells (HUVECs, \#LO CC-2519, Lonza, Basel, Switzerland) were cultured in Endothelial Cell Growth Basal Medium (EGM-2, \#C-22011, Bio-Connect, Huissen, The Netherlands). Human Mesenchymal Stem Cells (hMSCs) and human breast adenocarcinoma cells MDA-MB 231 were cultured in Dulbecco Modified Eagle Medium (DMEM, Lonza), supplemented with 10\% FBS and 1\% Penicillin/Streptomycin. hMSCs and MDA-MB 231 were expanded in 175- $\mathrm{cm}^{2}$ culture flasks (CELLSTAR® Filter Cap $175 \mathrm{~cm}^{2}$, GREINER BIO-ONE, Kremsmünster, Austria) in an incubator under standard humidified conditions $\left(37^{\circ} \mathrm{C}, 5 \% \mathrm{CO}_{2}\right)$. Endothelial cells were expanded in a $175-\mathrm{cm}^{2}$ culture flask (CELLSTAR $®$ Filter Cap $175 \mathrm{~cm}^{2}$ ) coated with collagen-I, by first incubating the flask with a collagen I solution at $0.1 \mathrm{mg} / \mathrm{mL}$ in $1 \mathrm{X}$ PBS for $1 \mathrm{~h}$ at $37^{\circ} \mathrm{C}$, and rinsing it with PBS. To collect cells, the flasks were rinsed with $15 \mathrm{~mL}$ of $1 \mathrm{X}$ PBS, incubated with $4 \mathrm{~mL}$ of a $0.25 \%$ $\mathrm{v} / \mathrm{v}$ Trypsin solution for $5 \mathrm{~min} .10 \mathrm{~mL}$ of culture medium was next added to stop the action of the trypsin, and the cells in suspension were collected. Next, the cells were centrifuged at 300 $\mathrm{g}$ for $3 \mathrm{~min}$, the supernatant was removed, and $2 \mathrm{~mL}$ of fresh medium was added. Cells were counted with a cell counter and partly reseeded in new culture flasks $(\sim 0.3$ million cells $/ \mathrm{mL})$. The remaining cells were kept for further experiments, as described in the following sections.

\subsection{LARGE BLOOD VESSEL FORMATION}

Following the VFP bottom-up approach described in the introduction, two cylindrical lumens were prepared in collagen $\mathrm{I}$ in the lateral channels of the microfluidic device. To ensure proper adhesion of the collagen to the channel walls, a surface pre-treatment step of the PDMS channel walls was performed, at room temperature. First, the channels were filled with a $3 \%$ v/v (3-aminopropyl) triethoxysilane (APTES, \#A3648, SIGMA-ALDRICH, Zwijndrecht, The Netherlands) solution in deionized water for $5 \mathrm{~min}$, and subsequently washed with $70 \%$ ethanol. Next, a 10\% glutaraldehyde (\#340855, SIGMA-ALDRICH) solution in PBS was introduced and incubated for $5 \mathrm{~min}$, and washed with ethanol (Figure 6-S2). The microfluidic devices were next heated at $60^{\circ} \mathrm{C}$ for $30 \mathrm{~min}$ to ensure complete ethanol evaporation. 

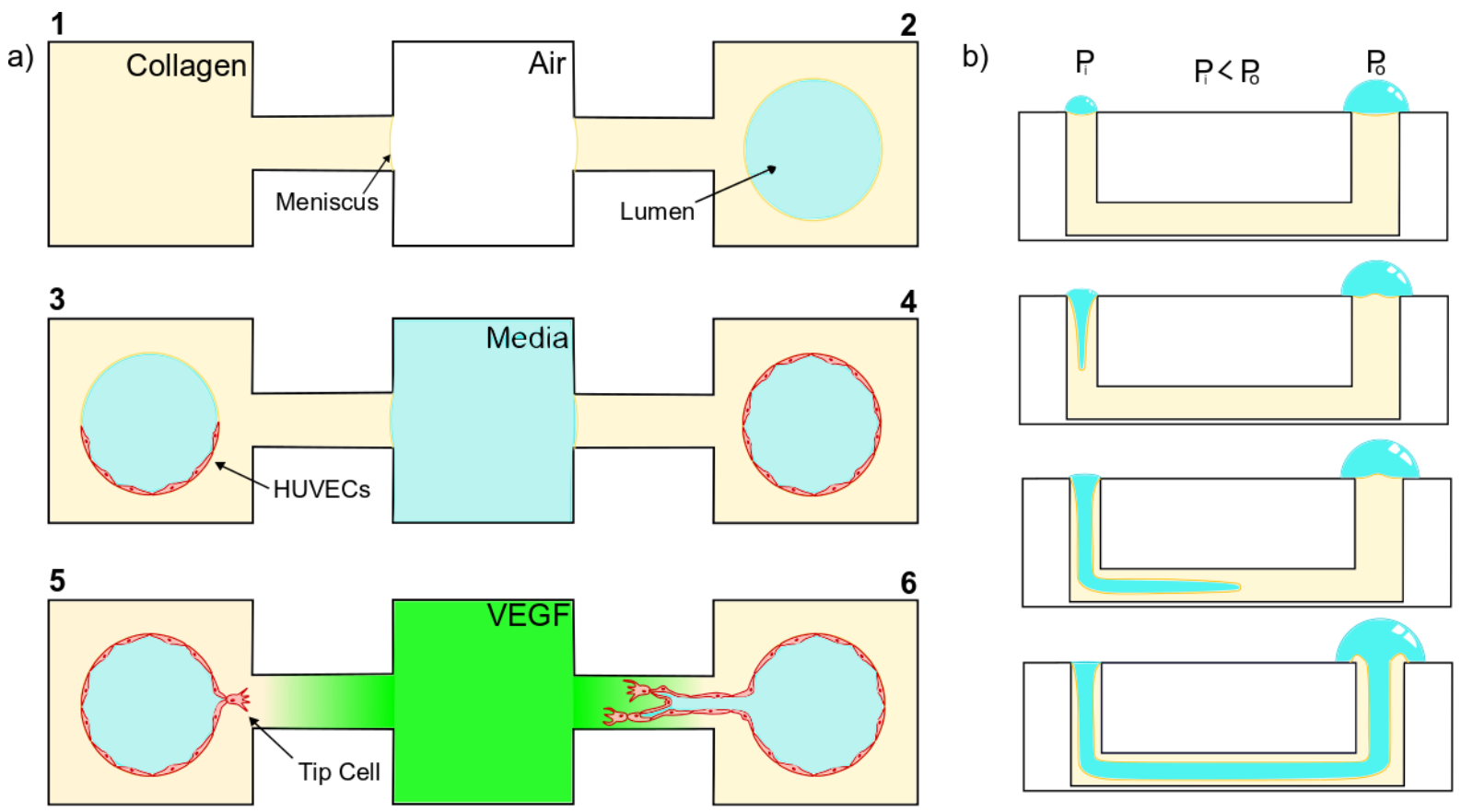

Figure 6-2 a) Complete protocol of the blood vessel formation and angiogenesis in the TME on-chip device. First, pre-cured collagen is poured in the lateral channels, also filling the connecting channels (1). Next, a lumen is formed using VFP (2). HUVECs are seeded twice, once with the device up straight (3), then once with the device flipped upside-down (4), to cover the entire lumen. Angiogenesis is triggered by injecting VEGF in the central channel, creating a gradient of concentration across the connecting channels (5), until complete neovascularization (6). b) Principle of the VFP method: A large drop of medium is deposited on the outlet of a microfluidic channel filled with pre-cured collagen. When adding a small droplet on top of the channel inlet, the internal pressure difference in each droplet causes the small droplet to get sucked in the channel, pushing collagen out via the outlet and creating a lumen in the hydrogel.

The lumen in collagen was formed using the VFP method. A high concentration of collagen I solution rat tail (\#354249, Corning, New York, USA) was mixed on ice with 10X PBS, DMEM media and sodium hydroxide $(\mathrm{NaOH})$, following Corning's instructions for ratios, to reach a final collagen concentration of $5 \mathrm{mg} / \mathrm{mL}$ with a $\mathrm{pH}$ of 7 . In some experiments, this collagen preparation was mixed with hMSCs at a concentration of 1 to 5 million cells $/ \mathrm{mL}$. In the latter case, the cells in suspension in medium were centrifuged, the supernatant removed, and collagen (on ice) added. The collagen mixture with or without hMSCs was injected in the two lateral channels of the microfluidic device, while also filling the connecting channels (Figure 6-2.a.1). A $20 \mu \mathrm{L}$ droplet of EGM-2 medium (at $5^{\circ} \mathrm{C}$ ) was then immediately deposited on both lateral channels' outlets. After $10 \mathrm{~s}$ curing of the collagen in the channels at room temperature, a series of 10 droplets of $c a .2 \mu \mathrm{L}$ were successively deposited on both lateral 
channels' inlets. The resulting difference in internal pressure between the two droplets $(2 \mu \mathrm{L}$ vs. $20 \mu \mathrm{L}$ ) resulted in the creation of a flow in the channel (a phenomenon known as passive pumping [14]) inside the collagen matrix, pushing the uncured collagen from the center of the channel out the system via the outlets (Figure 6-2.b). This approach resulted in the formation of a collagen lumen of $c a .400 \mu \mathrm{m}$ in diameter (Figure 6-2.a.2). The microfluidic device was next placed in the incubator for $30 \mathrm{~min}\left(37^{\circ} \mathrm{C}, 5 \% \mathrm{CO}_{2}\right)$ to complete the collagen gelation, after which the central channel was filled with either medium or collagen containing a tumour spheroid as discussed later. The medium chosen here, as well as in all co-culture experiments, was a DMEM:EGM-2 mixture in a 1:1 ratio, based on a viability assay performed on monolayers of HUVECs and hMSCs (Figure 6-S1).

Finally, to finalize the preparation of the two large blood vessels, HUVECs were seeded at a concentration of 15 million cells/mL (in EGM-2) to fill both lumens ( $c a .4 .5 \mu \mathrm{L}$ per lumen), and incubated for $1 \mathrm{~h}\left(37^{\circ} \mathrm{C}, 5 \% \mathrm{CO}_{2}\right)$ (Figure 6-2.a.3). The lumens were next washed by pipetting fresh medium to eliminate the excess of cells not adhering to the collagen walls. HUVECs were next seeded in the same manner, and the microfluidic device was flipped to allow cells to adhere on the top part of the lumen. The microfluidic device was placed in the incubator for $1 \mathrm{~h}\left(37^{\circ} \mathrm{C}, 5 \% \mathrm{CO}_{2}\right)$, after which medium was refreshed one last time (Figure 62.a.4).

\subsection{TUMOUR SPHEROID FORMATION AND CULTURE}

Multicellular tumour spheroids were prepared as described in chapter 3. A $1 \% \mathrm{w} / \mathrm{w}$ Pluronic F-127 (\#P2443, SIGMA-ALDRICH) solution in DI water was placed in a Petri dish patterned with an array of microwells $(800 \mu \mathrm{m}$ in diameter, $500 \mu \mathrm{m}$ in height), incubated overnight $\left(37^{\circ} \mathrm{C}\right)$, and replaced with a mixture of DMEM and EGM-2 in a 1:1 volume ratio. MDA-MB231, hMSCs, HUVECs and cells were mixed in one of the following ratios: 1:0:1, 2:0:1, 4:0:1, 5:0:1, 1:1:0, 4:1:1, and 4:2:1, and the resulting cell mixture was introduced in the Petri dish. The cells were incubated $\left(37^{\circ} \mathrm{C}, 5 \% \mathrm{CO}_{2}\right)$ and medium refreshed every 2 days. After 4 days of incubation, the spheroids were pipetted in an Eppendorf tube.

\subsection{VEGF GRADIENT GENERATION}

First, to estimate the possibility of generating a gradient of concentration in the connecting channels, a 2D COMSOL simulation was performed, limiting the model to half of the microfluidic device taking into account the axial symmetry with respect to the central 
channel. Transport and diffusion of VEGF were simulated using the "Laminar Flow" and "Transport of Diluted Species" modules. $20 \mathrm{ng} / \mathrm{mL}$ of VEGF was perfused in the central channel at a flow-rate $0.1 \mu \mathrm{L} / \mathrm{min}$ for $24 \mathrm{~h}$ at $37^{\circ} \mathrm{C}$. The transport of VEGF in collagen In the device was estimated with a diffusion coefficient of $6 \times 10^{-11} \mathrm{~m}^{2} / \mathrm{s}$ [24]. The "Darcy's law" module was used to model the properties of the collagen hydrogel with a porosity of $\varepsilon=0.996$ and a permeability of $\kappa=5.5 \times 10^{-14} \mathrm{~m}^{2}[25,26]$. The lateral channels were treated as reservoirs with no outlet (lateral channel closed) of collagen, in which VEGF could freely diffuse (Figure 6-S2).

Experimentally, to induce sprouting and formation of new blood vessels in the connecting channels, a $20 \mathrm{ng} / \mathrm{mL}$ solution of human recombinant (Thermo Fisher Scientific, Waltham, MA, USA) in a 1:1 vol. ratio DMEM:EGM-2 media was perfused in the central channel. This media was perfused at $0.1 \mu \mathrm{L} / \mathrm{min}$ for 4 days using a syringe pump (Elite 11, Harvard Apparatus, Cambridge, MA USA). To prevent VEGF saturation in the lateral channels and maintain a stable gradient in the connecting channels, the lateral channels were refreshed daily by pipetting $20 \mu \mathrm{L}$ of VEGF-free medium.

\subsection{StAining ANd IMAging}

\subsubsection{CellTracker}

To easily distinguish HUVECs and hMSCs in the tumour spheroid, these cells were both first stained with CellTracker dyes prior to spheroid formation. To do so, HUVECs and hMSCs were collected from the culture flasks into Eppendorf tubes and centrifuged. $10 \mu \mathrm{M}$ solutions of CellTracker green (5-chloromethylfluorescein diacetate, CMFDA, Thermo Fisher Scientific) and CellTracker red (CMTPX, Thermo Fisher Scientific) were prepared in media. The cell medium in the Eppendorf tubes containing the cells in pellets was replaced by the medium supplemented with the CellTracker dye in which cells were resuspended and incubated for $30 \mathrm{~min}\left(37^{\circ} \mathrm{C}, 5 \% \mathrm{CO}_{2}\right)$. The cells were then centrifuged, washed three times with medium, and used to prepare spheroids.

\subsubsection{Viability assay}

Cell viability in the microfluidic devices was assessed, before and after neovascularization, using a standard live/dead assay, and a mixture of $4 \mu \mathrm{g} / \mathrm{mL}$ Calcein-AM (\#L3224, Thermo Fisher Scientific) and $2 \mu \mathrm{g} / \mathrm{mL}$ Ethidium Homodimer-1 (EthD-1, \#L3224, Thermo Fisher Scientific). $20 \mu \mathrm{L}$ of this mixture in medium was pipetted in the three different inlets of the 
device and incubated for $30 \mathrm{~min}\left(37^{\circ} \mathrm{C}, 5 \% \mathrm{CO}_{2}\right)$. Fluorescence microscopy images were acquired immediately afterward.

\subsubsection{Immunostaining}

Immunostaining was performed by fixating the cells with a $4 \%$ formaldehyde solution for 30 min (RT) followed by a permeabilization step with 1\% Triton X-100 in PBS (PBST) for $1 \mathrm{~h}$. Adherens-junction proteins between HUVEC cells in the large blood vessels (lateral channels) were stained by pipetting $20 \mu \mathrm{L}$ of anti-Vascular Endothelial-Cadherin (VECadherin) primary antibody $(10 \mu \mathrm{g} / \mathrm{mL}$ in Bovine Serum Albumin (BSA, \#36-1900, Thermo Fisher Scientific) inside the channels, and incubating the device overnight $\left(5^{\circ} \mathrm{C}\right)$. The Primary antibody staining solution was washed away by pipetting the channels $3 \mathrm{X}$ with $1 \mathrm{X}$ PBS. Next, $20 \mu \mathrm{L}$ of secondary antibody staining, conjugated to Alexa Fluor $488(2 \mu \mathrm{g} / \mathrm{mL}$ in BSA, \#A11034, Thermo Fisher Scientific), was pipetted in the lateral channels, and incubated for $3 \mathrm{~h}$ (RT). HUVEC cellular conformation was evaluated using Phalloidin (ActinGreen ${ }^{\text {TM }} 488$ ReadyProbes, Thermo Fisher Scientific) to stain actin filaments. Hoechst (NucBlue, Thermo Fisher Scientific) was used to counterstain the cell nuclei.

\subsubsection{Image acquisition}

Low resolution images of the spheroid formation and blood vessel leakiness experiments were acquired using an EVOS-FL microscope (Thermo Fisher Scientific), with an RFP led cube to observe red signals, a GFP led cube for the green signal, and a DAPI led cube for the blue signal. High resolution images of the large blood vessel formation and the neovascularization were acquired using a confocal microscope (Nikon A-1R, Nikon, Tokyo, Japan) with a 20x objective and a thickness of acquisition of $c a .20 \mu \mathrm{m}$. 


\section{ReSUltS AND DiscuSSIONS}

\subsection{TUMOUR VASCULARIZATION APPROACH}

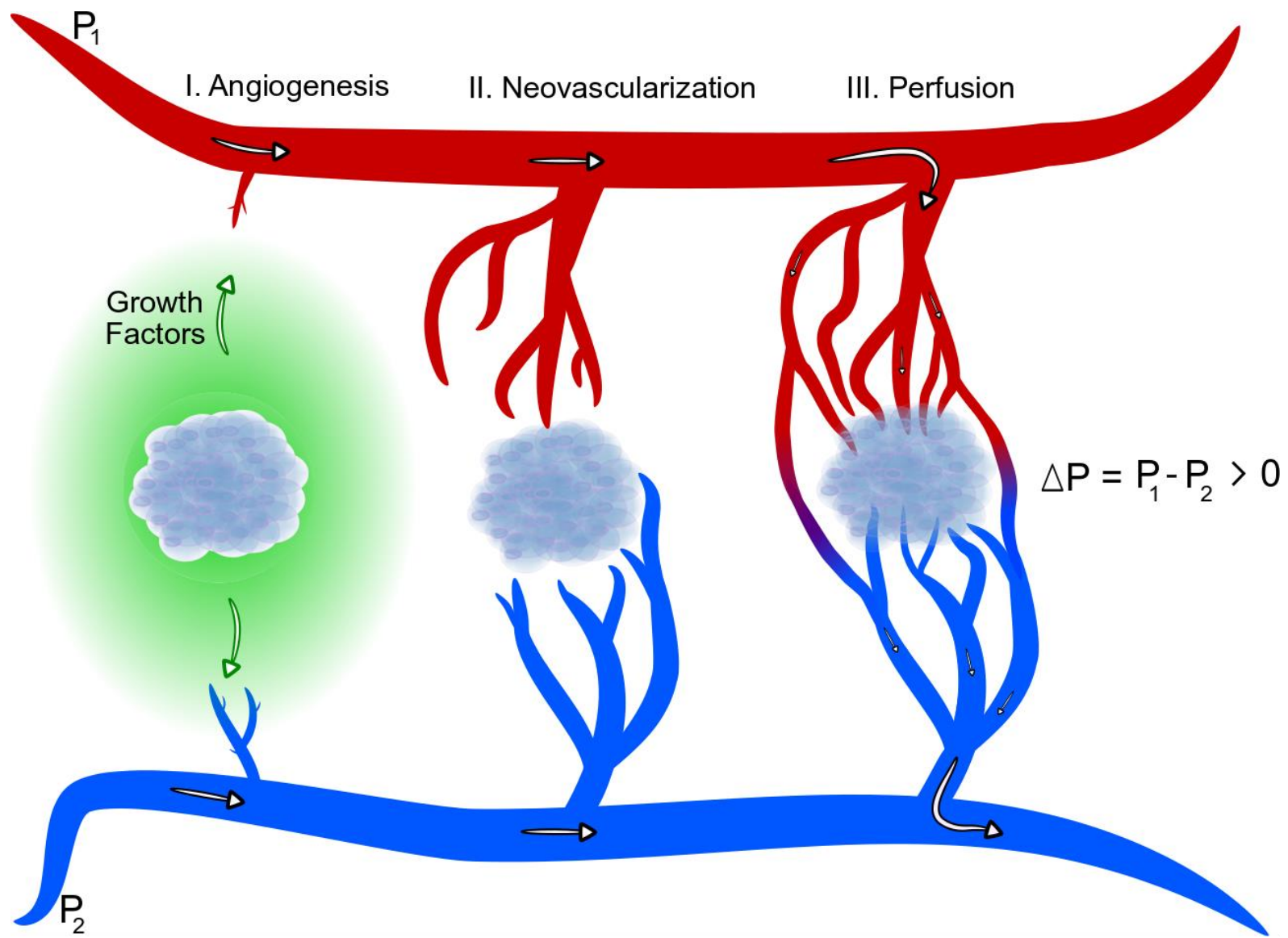

Figure 6-3 Creation of a vascularized tumor-on-chip model: a tumor spheroid is placed between two large pre-formed blood vessels, and, in presence of cancer-secreted growth factors $(G F)$, or manually injected GF near the spheroid, angiogenesis occurs (I). Neovascularization forms form the large preformed blood vessels, toward the tumor spheroid (II). When they meet the spheroid and each other, both neovasculatures create a closed circuit between the lateral blood vessels (III). The capillary network becomes perfusable, by applying a difference of pressure $(\triangle P)$ between the lateral blood vessels, much like an arteriole (red) and a vein (blue) would in vivo.

In this chapter we aimed at reproducing the cancer-induced angiogenesis process, which, as explained previously, is the formation of new blood vessels from previously existing ones, which is steered in vivo by the presence of a tumour [27]. As such, we developed a platform which comprises a single tumour spheroid, potentially secreting pro-angiogenic factors. This spheroid is placed between two large and healthy blood vessels representing an arteriole and a 
vein (same diameter, but different pressures). Growth factors secreted by the tumour spheroid and/or manually injected between the large blood vessels, are expected to stimulate cellular migration and neovascularization toward the tumour, and from both blood vessels (Figure 6-3) $[17,19,24,28,29]$. Once the newly formed capillaries reach the tumour, they connect the two blood vessels, together with the tumour. Once the vasculature network completed, flow can be induced in the new capillary vessels by applying a difference of pressure between the two large blood vessels, to supply the tumour with nutrients as depicted in Figure 6-3.

\subsection{TUMOUR SPHEROID FORMATION}

\subsubsection{Human breast cancer spheroid model}

The tumour spheroids were prepared as described in the previous chapters, by combining cell types found in the in vivo tumour microenvironment: Human Breast Carcinoma cells MDA-MB231, human Mesenchymal Stem Cells (hMSCs), and Human Umbilical Vein Endothelial Cells (HUVECs). As before, to recreate the gradient of nutrient/oxygen depletion, large spheroids ( $c a .400 \mu \mathrm{m}$ in diameter) were prepared.

In a first instance, to promote the formation of small blood vessels inside a tumour spheroid, HUVECs were directly incorporated and mixed with the cancer cells (MDA-MB 231) in a MDA-MB 231:HUVECs 1:1 or 2:1 ratio. After $48 \mathrm{~h}$ incubation, the resulting spheroids presented very little compactness, many cells being released from the aggregates into the suspension (Figure 6-4.a). This poor miscibility of the cells could be explained by the natural expression of growth factors by HUVECs (e.g., Ang-2) inhibiting cell-cell interactions in a hypoxic environment $[18,30]$. By changing the cellular ratio and increasing the amount of cancer cells to MDA-MB 231:HUVECs 4:1 and 5:1, more stable and spherical tissues of $c a$. $320 \mu \mathrm{m}$ in diameter were formed, and almost no single cell was found in suspension (Figure 64.b). To still ensure enough HUVECs would be present in the spheroids, a MDA-MB 231:HUVECs ratio of 4:1 was retained.

Two of the cell types highly present in the TME are the smooth muscle cells, normally acting as physical support for the blood vessel, and the cancer associated fibroblasts, which notably secrete growth factors and Extracellular Matrix (ECM). A third type of cells present in the TME are the mesenchymal stem cells (hMSCs). These multipotent stromal cells can, through exposure to specific growth factors (e.g., Transforming Growth Factor beta (TGF- $\beta 1$ ) 
[31]), differentiate into either smooth muscle cells (SMCs) or cancer associated fibroblasts [3234]. To limit the complexity of our TME model, we hypothesized that hMSCs could both act as SMCs to support the neovascularization, and as CAFs inside the spheroid to promote ECM production.

As a first model, in a microwell array (microwells of $800 \mu \mathrm{m}$ in diameter and $500 \mu \mathrm{m}$ in depth), spheroids with a MDA-MB 231:hMSCs 1:1 composition were prepared, which were ca. $248 \pm 47 \mu \mathrm{m}$ in size. These spheroids presented smooth surfaces with no cell in suspension, indicating strong cell-cell interactions (Figure 6-4.c). Therefore, this tumour cell: MSCs ratio was kept for the triple co-culture model. Spheroids with a MDA-MB231:hMSCs:HUVECs in a 4:1:1 and 4:2:1 ratio were prepared as before, yielding tissues of $c a$. $280 \pm 17 \mu \mathrm{m}$ in size (Figure 6-4.d).

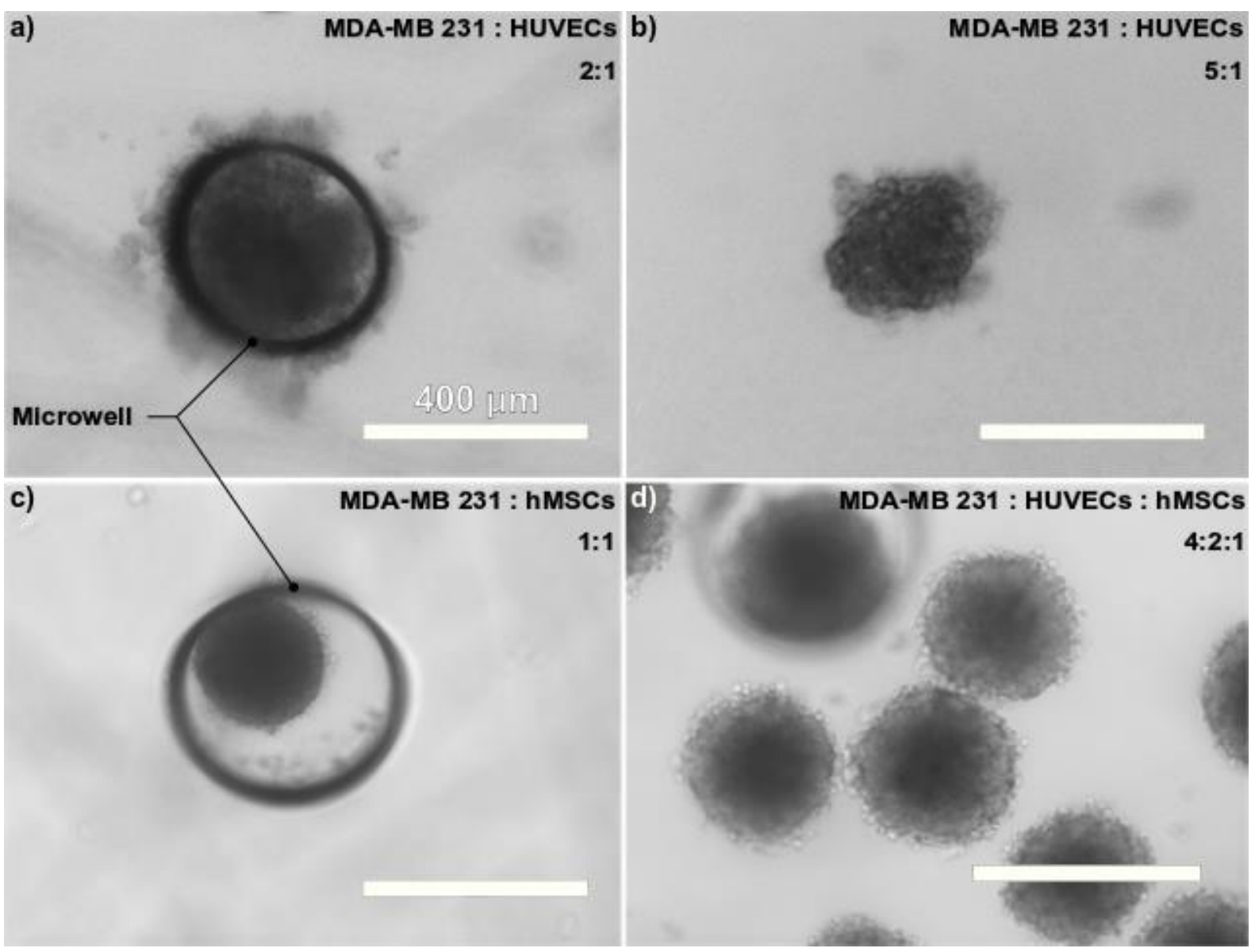

Figure 6-4 Spheroid formation in a microwell array (microwells of $800 \mu \mathrm{m}$ in diameter, $500 \mu \mathrm{m}$ depth) with different cellular ratios of MDA MB-231, HUVECs and hMSCs: (a) MDA-MB 231: HUVECs in a ratio of 2:1 (b) MDA-MB 231: HUVECs in a ratio of 5:1 (c) MDA-MB 231: hMSCs in a ratio of 1:1, and (d) MDA-MB 231: HUVECs : hMSCs in a ratio of 4:2:1. 


\subsubsection{Tumour spheroid cellular organization}

To characterize the cellular organization within the tissue, cells were stained with CellTracker before MCTS formation. After $48 \mathrm{~h}$ incubation in the microwell array, fluorescent images of the resulting spheroids were acquired. In the MDA-MB 231: HUVECs spheroids ( $4: 1$ or $5: 1$ ratio), the cell distribution appeared to be random (Figure 6-5.a), but in the MDAMB 231: hMSCs spheroids in a 1:1 ratio, the hMSCs (green) seemingly shifted to the outer layer of the spheroid (Figure 6-5.b).
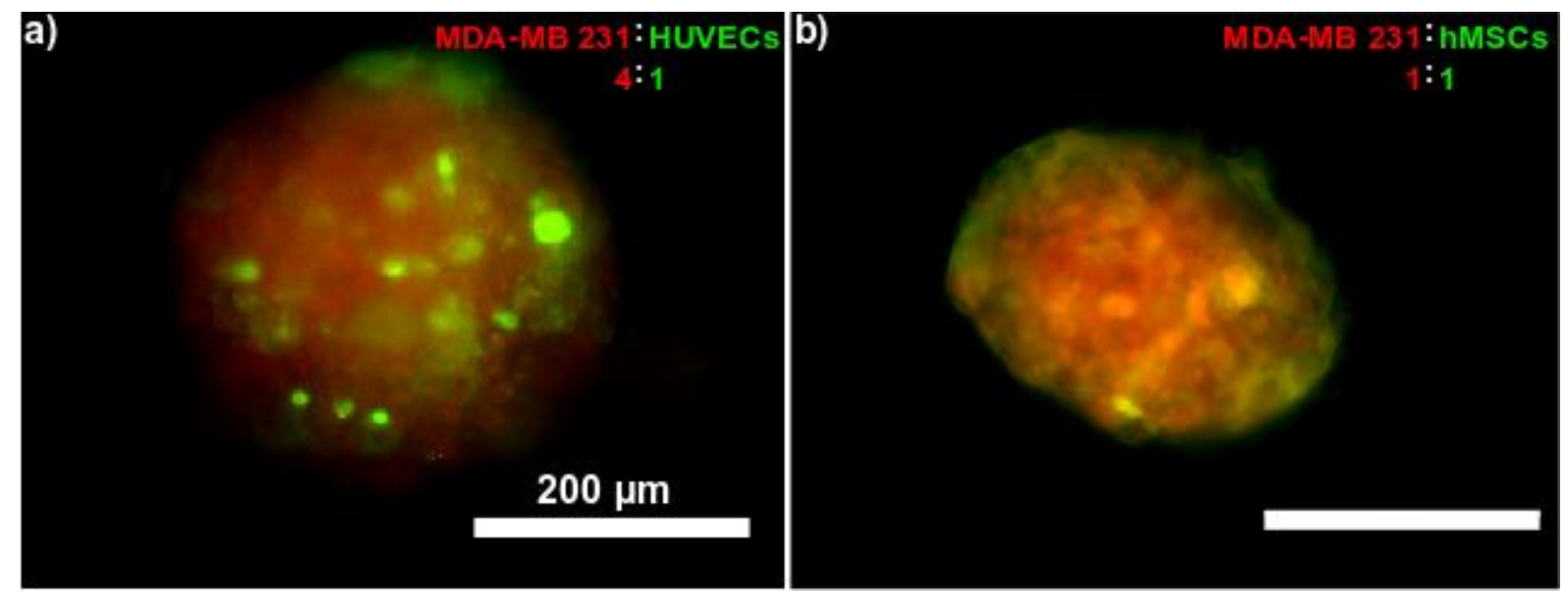

Figure 6-5 Spheroids aggregated in the microwell array, from cells stained with CellTracker. a) MDAMB 231: HUVECs spheroids in a 4:1 ratio. b) MDA-MB 231: hMSCs spheroids in a 1:1 ratio. Here hMSCs are found at the periphery of the spheroids.

\subsection{LARGE BLOOD VESSEL FORMATION AND CHARACTERIZATION}

\subsubsection{Single blood vessel formation}

The viscous finger patterning (VFP) method, developed by Bischel et al. [13] was implemented to generate large lumens in a collagen matrix in the lateral channels. As a first step, the protocol was tested and optimized using single straight or curved channels with a square cross-section of $600 \times 600 \mu \mathrm{m}^{2}$. Lumens of $399 \pm 56 \mu \mathrm{m}$ (from $\mathrm{n}=12$ lumens) in width were generated (Figure 6-6.a). The presence of a curvature in the channel did not affect the lumen formation and dimensions; the lumen position only slightly shifted inside the curvature, compared to the straight channel, leading to a localized variation in the collagen wall thickness.

To evaluate the circularity of the lumen, HUVECs cells were stained with CellTracker and injected in the channels, as described in the method section. After adhesion of the cells to the collagen, using confocal imaging, a 3D reconstruction of the generated blood vessels was 
created from which a cross section was extracted (Figure 6-6.b). The rather elliptic shape of the resulting blood vessel is assumed to be caused by the collagen collapsing right after the creation of the lumen, before its complete gelation.

To simulate the presence of smooth muscle cells in the system and support the blood vessels, hMSCs were mixed with the collagen prior to its injection in the channel, with varying concentrations. After 4 days of culture, a live/dead assay was performed: in a device with a HUVEC-only blood vessel (no hMSCs in the collagen), or with hMSCs in collagen only (no HUVECs in the lumen), (Figure 6-6.c). In the first case, no dead cells were found. The main possible reasons for this was that the dead cells detached from the collagen lumen and were flushed out of the channels with the flow (e.g., from refreshing the medium). In the second case, when hMSCs cells were seeded at 1,2 or 3 million cells $/ \mathrm{mL}$ in the collagen, the viability assay indicated a very high cell survival, with the live cells (green) stretching in the collagen matrix. When the concentration was increased to 5 million cells $/ \mathrm{mL}$, the viability dropped, and most hMSCs were found dead (red), they were circular and trapped in the hydrogel. It was hypothesized that at a high cell concentration, refreshing the medium once every day in the channel was insufficient to supply the cells with enough nutrients.

\subsubsection{Blood vessel characterization}

The HUVECs endothelial barrier was characterized via immunostaining of the adherensjunction protein VE-cadherin. Imaging revealed tight junctions between the cells, indicating a strong endothelial barrier (Figure 6-6.d). However the cells presented no specific organization: in vivo they are stretched by the shear stress induced by the blood flow (vein $=0.1-0.5 \mathrm{~Pa}$ ), and align in its direction $[35,36]$. Here, to obtain similar results, we estimated the flow-rate necessary to achieve comparable shear stress. In a cylinder, the wall shear stress (WSS) equation can be expressed as:

$$
\text { WSS }=\mu * \frac{8 * V m}{D}
$$

where $\mu$ is the dynamic viscosity of the media, $D$ the diameter of the lumen and $V_{m}$ the flow mean velocity [35, 37]. Blood being more viscous than water $\left(2.78\right.$ vs. $0.72 \mathrm{mPa} \mathrm{s}^{-1}$, respectively, at $37^{\circ} \mathrm{C}$ ), the flow-rate required in the microfluidic device would be much higher than in vivo, and to achieve a wall shear stress of $c a .0 .1 \mathrm{~Pa}$ in a cylinder of $400 \mu \mathrm{m}$ in diameter, media should be perfused at $c a .1 \mathrm{~mL} / \mathrm{h}$. 

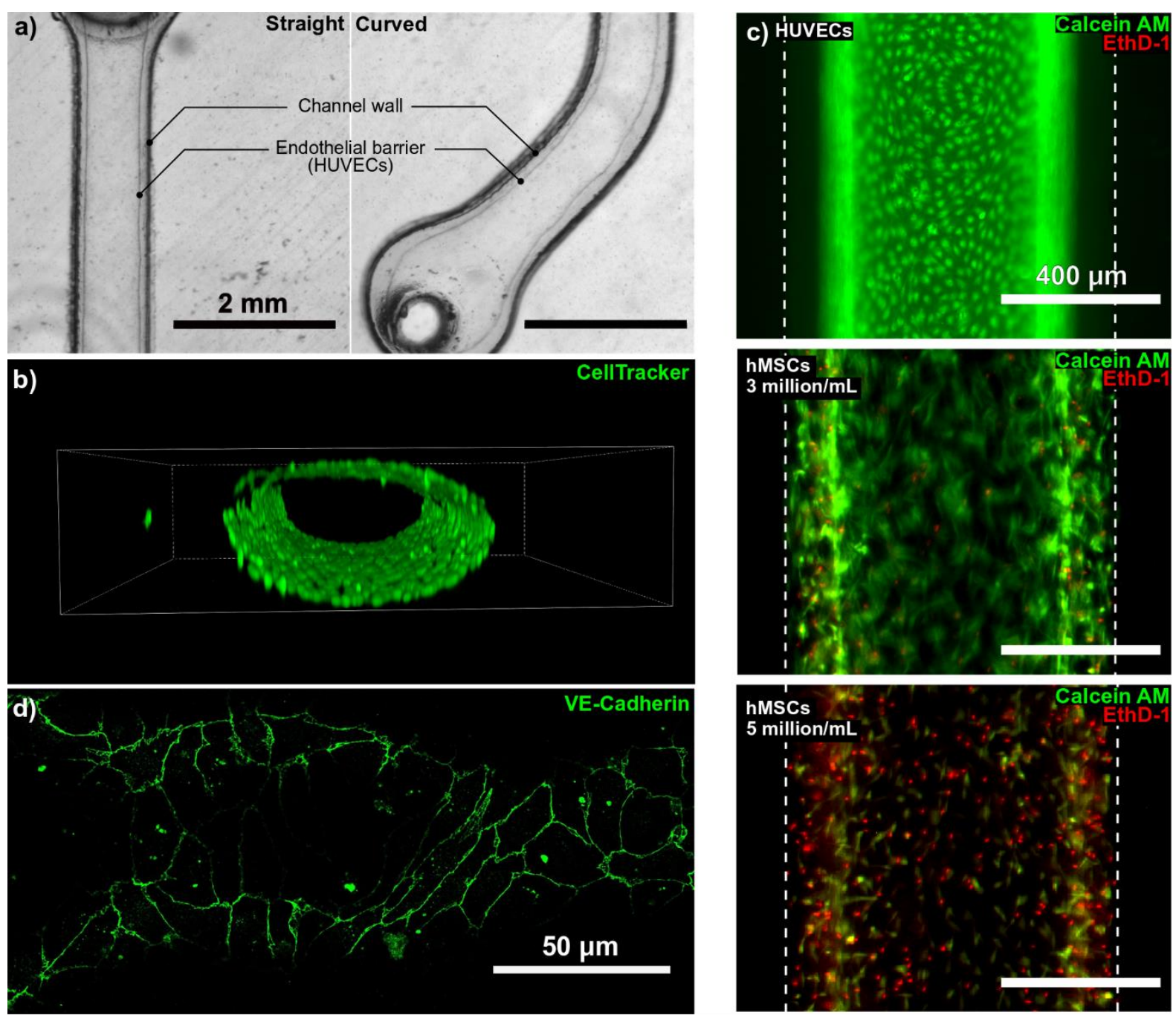

Figure 6-6 Characterization of single large blood vessels. a) Lumen formation and cell seeding in straight or curved channels. Lumens with parallel walls are formed, independently of the presence the channel geometry. b) 3D reconstruction of a blood vessel from cross sections (cells being stained with CellTracker). c) Live/Dead assay of HUVECs 7 days after seeding in the collagen lumen (top), and hMSCs only, in the collagen matrix, at 3 million cells $/ m L$ (middle) and 5 million cells $/ m L$ (bottom). $d$ ) Imaging of the adherens junction proteins between HUVEC cells in the blood vessel, using VECadherin antibody.

After formation of the endothelial barrier in our device, the channel was perfused with medium at $1 \mathrm{~mL} / \mathrm{h}$. After $24 \mathrm{~h}$ perfusion, the cells present in the channels indicated stretching in the direction of the flow, but large sections of the cells forming the endothelial wall were missing (Figure 6-S3.a). This could be explained by the impact of the shear stress on the thick collagen present outside the blood vessels. Indeed, in vivo, the deformation caused by the shear stress would be dampened by the presence of many other cells supporting the endothelial cells 
(e.g., smooth muscle cells), and in the blood (e.g., erythrocytes). In contrast in our microfluidic device, the thick layer of collagen was unable to sustain the stress and cracks quickly appeared with cells being detached from the walls. By reducing the flow-rate down to $0.1 \mathrm{~mL} / \mathrm{h}$, cells could safely and continuously be perfused without endangering the structure of the blood vessels (Figure 6-S3.b), while promoting a change in cell conformation (Figure 6-S3.c).

\subsection{Tumour MicroenvironMENT ON CHIP}

\subsubsection{Microfluidic design}

To realize the system presented in section 4.1, a single spheroid of $c a .360 \mu \mathrm{m}$ in diameter was inserted in a microfluidic device in a straight channel of $500 \mu \mathrm{m}$ in width and $600 \mu \mathrm{m}$ in height. This channel included a constriction acting as a trapping structure for a single MCTS. The large blood vessels were prepared on both sides of this "central channel", in two "lateral channels" of $600 \times 600 \mu \mathrm{m}^{2}$. Finally, the central and lateral channels were joined with two 500 $\mu \mathrm{m}$ long "connecting channels", where neovascularization would next occur.

By aligning two PDMS structures to form the microfluidic device, the connecting channels were placed at the half-height of the lateral and central channels. This way, neovascularization was initiated at the half-height of the large blood vessels, optimized to progress laterally toward the tumour. The dimensions of the connecting channels were chosen to favor collagen injection in the connecting channels without filling the central channel, by testing designs with different connecting channels dimensions (data not shown). The dimensions retained to achieve this, using a $5 \mathrm{mg} / \mathrm{mL}$ collagen I solution, were $200 \mu \mathrm{m}$ in height and $400 \mu \mathrm{m}$ in width. When injecting collagen, capillary forces drove the filling of the lateral channel and the connecting channel: as a result, in the connecting channels, a concave meniscus formed at the interface collagen-air (Figure 6-7.b). The contact angle at the meniscus was affected by the amount of force applied when pipetting the collagen in the lateral channel, adding to the capillary forces. The maximum contact angle obtained was $\theta=50^{\circ}$, going down with the force exerted while pipetting, and reached $\theta=0^{\circ}$ (Figure 6-7.b). When too much force was exerted, the collagen filled the central channel.

\subsubsection{Blood vessel parallelization}

The large blood vessels were prepared in the TME microfluidic device in the same manner as described in section 4.3. Two lumens were generated in the lateral channels and after a short incubation time ( $c a .5 \mathrm{~min}$ ), the middle channel was filled with medium to prevent 
the collagen in the connecting channel from drying. When let to dry for a longer period of time, the collagen present at the interface collagen-air would densify, causing issues later on, by acting as a barrier to the neovascularization.

Interestingly, when the VFP was performed in the lateral channels, a local protuberance was created in the lumen, at the level of connecting channels, due to the absence of a wall at that location (Figure 6-7.b). The protuberance length varied with the collagen pre-curing time (before the VFP was performed), reaching up to $100 \mu \mathrm{m}$ in length. This "imperfection" in the lumen structure was advantageous, as it has been reported that vessel sprouting is favored at such irregularities in vivo (e.g., blood vessel branching) [38].

After HUVEC seeding, and incubation until 100\% coverage of the lumen by the cells, the permeability was characterized in a quantitative manner. FITC-dextran ( 1 ize $=40 \mathrm{kDa}$ ) was perfused inside the blood vessels at a flow-rate of $0.1 \mu \mathrm{L} / \mathrm{min}$. After $45 \mathrm{~min}$, the fluorescent dye did not locally leak outside the blood vessels, indicating a strong endothelial barrier (Figure 6-7.c). Longer experiment could not be performed as the collagen was gradually stained from the inlets and outlets through diffusion, preventing the observation of localized leakage.

\subsubsection{Angiogenesis and neovascularization}

Prior to injecting the tumour spheroid in the system, to complete the TME microfluidic device, a characterization was performed to confirm the possibility of triggering angiogenesis in the device. First, a COMSOL simulation was performed to evaluate the VEGF gradient in the connecting channels (Figure 6-8.a). This simulation, in which the diffusion properties in collagen were incorporated, displayed the formation of a gradient in the connecting channel, maintained after $24 \mathrm{~h}$ of perfusion of VEGF (42 kDa) at $20 \mathrm{ng} / \mathrm{mL}$ at $1 \mu \mathrm{L} / \mathrm{min}$ (Figure 6-S4). Therefore, it was hypothesized that this gradient could be maintained indefinitely by refreshing daily the lateral channels.

Experimentally, VEGF was added after complete formation of the large blood vessels (after $c a .48 \mathrm{~h}$ ). Perfusion was applied at $0.1 \mu \mathrm{L} / \mathrm{min}$ for 4 days in absence of hMSCs in the collagen matrix. After $24 \mathrm{~h}$ perfusion, sprouting was observed at the intersection of the lateral channels and the connecting channels, stirring toward the central channel. After 4 days, more sprouting could be found and cells migrated deeper in the collagen reaching up to $180 \pm 20 \mu \mathrm{m}$ in length (Figure 6-8.b). However, no capillary vessel was formed and the migrating cells were isolated. The lack of cell-cell interaction between the endothelial cells was hypothesized to be caused by missing growth factors (e.g., Fibroblast Growth Factors (FGF) or Angiopoietin-1 
(ANG-1)) secreted by other cells in the TME (e.g. smooth muscle cells, cancer associated fibroblasts).
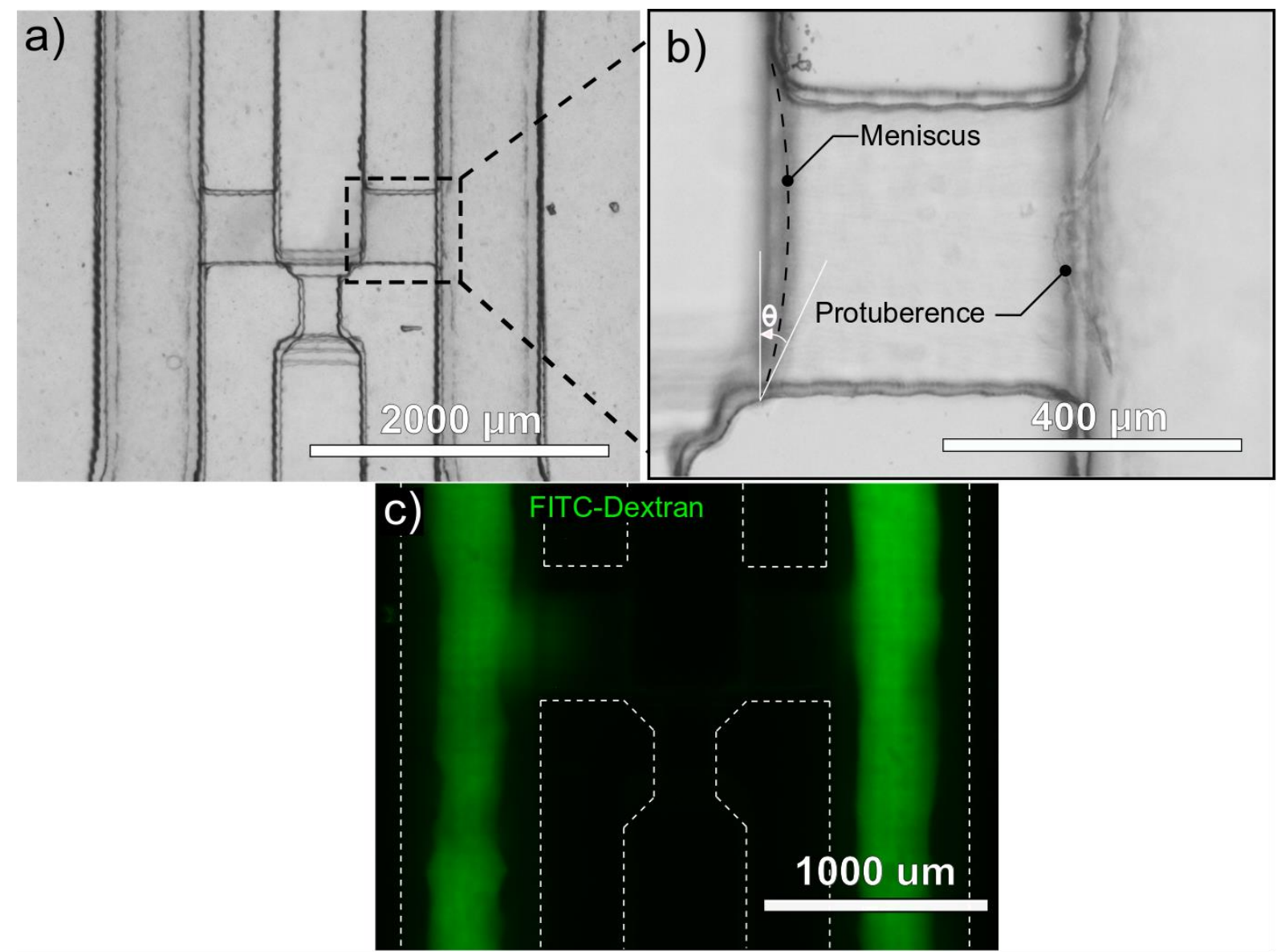

Figure 6-7 Blood vessel characterisation in TME on-chip device a) TME on-chip device, with large blood vessels produced in both lateral channels. b) Connecting channel filled with collagen, with the capillary forces induced meniscus, and the VFP induced protuberance. c) Characterization of endothelial barrier permeability, by perfusing FITC-Dextran (40 kDa) with a syringe pump at 0.1 $\mu \mathrm{L} / \mathrm{min}$ for $45 \mathrm{~min}$. Image acquired after $30 \mathrm{~min}$.

Therefore, in a second series of experiments, hMSCs were added to the collagen matrix (3 million cells/mL) to supply the HUVECs with growth factors and provide physical support. In these latter experiments, after 4 days, new capillary vessels formed with a higher density and reached the central channel $(>500 \mu \mathrm{m})$. Altogether, these experiments demonstrated that hMSCs can act as a catalyst for the neovascularization, most probably through the secretion of growth factors, such as Fibroblast Growth Factors (FGF), which has been reported to be also 
produced by the smooth muscle cells, and known to be essential to the formation of stable vessels in vivo [39] .

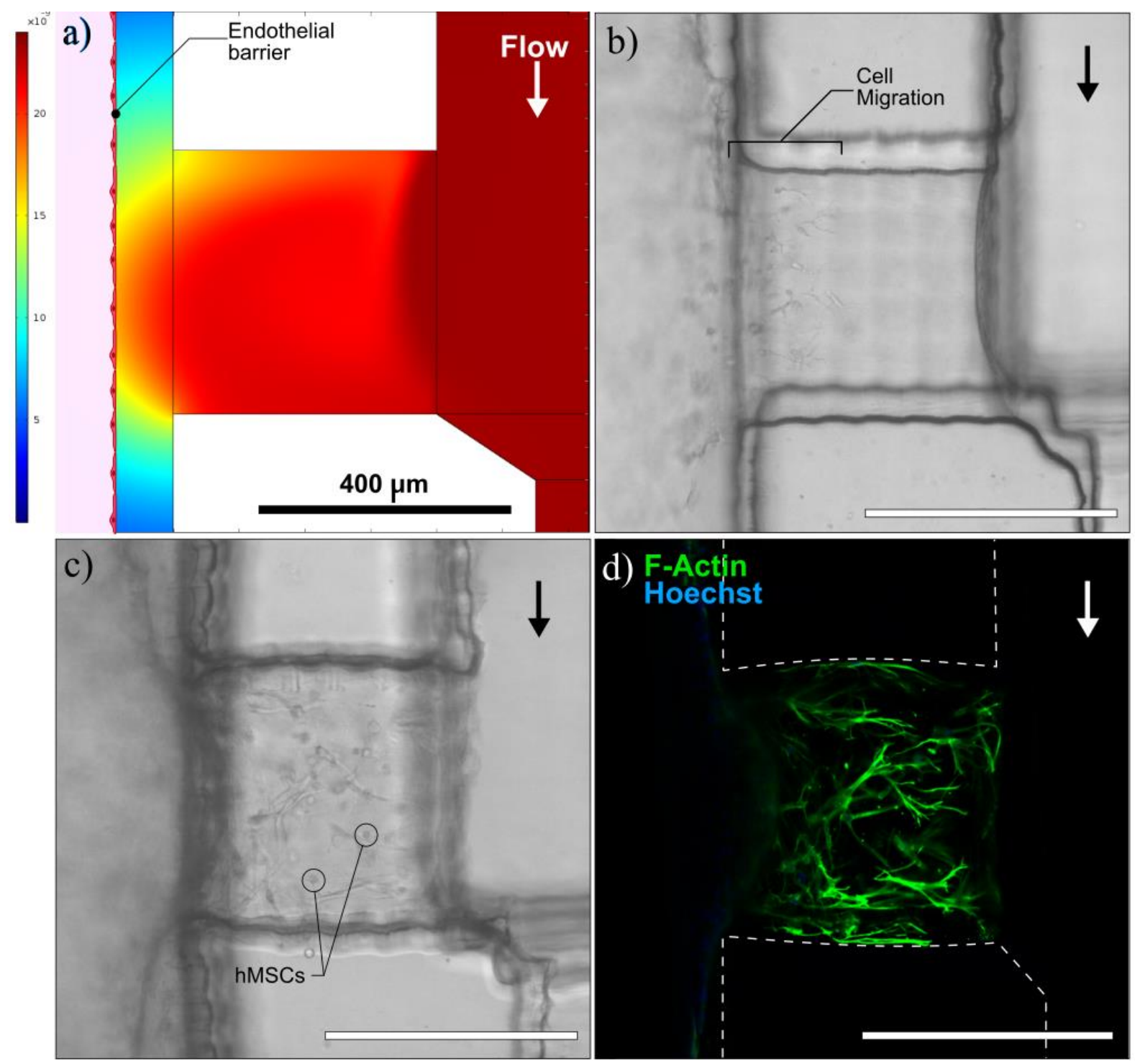

Figure 6-8 Neovascularization in the connecting channels. a) COMSOL simulation of VEGF diffusion, perfused in the central channel at $0.1 \mu \mathrm{L} / \mathrm{min}$, after $24 \mathrm{~h}$. b) Image of HUVECs sprouting after 4 days of VEGF perfusion. c) Image of HUVECs sprouting in the presence of hMSCs inside the collagen matrix, after 4 days of VEGF perfusion. d) Confocal image of (c) after fixation of the cells, and F-actin (green) immunostaining and Hoechst (blue) counterstaining. Scale bars $=400 \mu \mathrm{m}$.

\subsubsection{Tumour spheroid injection}

The next step was to combine this vascular system, comprising two large blood vessels and a neovasculature, together with a tumour spheroid. In a first approach the tumour spheroid would be added after the large blood vessel formation and neovascularization has taken place. 
As a first experiment, in a microfluidic device containing two large blood vessels ( $48 \mathrm{~h}$ after HUVECs seeding), a tumour spheroid was trapped in the constriction in the central channel, in collagen I (Figure 6-9.a). The collagen hydrogel was gelated as previously, but after $24 \mathrm{~h}$ incubation $\left(37^{\circ} \mathrm{C}, 5 \% \mathrm{CO}_{2}\right)$, its configuration changed. Indeed, the collagen in the central channel was detaching from the channel walls and from the collagen present in the connecting channels (Figure 6-9.b). Most likely, the APTES-glutaraldehyde coating originally present on the central channel walls had been covered over time by proteins or other molecules present in the media or secreted by the cells. Additionally, detached collagen appeared to be contracted, and stretched toward the spheroid, as seen as a darkening of the collagen structure near the spheroid. The cells present in the spheroid pulled on the collagen fibers, inducing the deformation of the collagen hydrogel [27]. Since the APTES-glutaraldehyde coating could not be applied again due to the toxicity of the free glutaraldehyde, a fibronectin coating was tested, giving yet the same results.
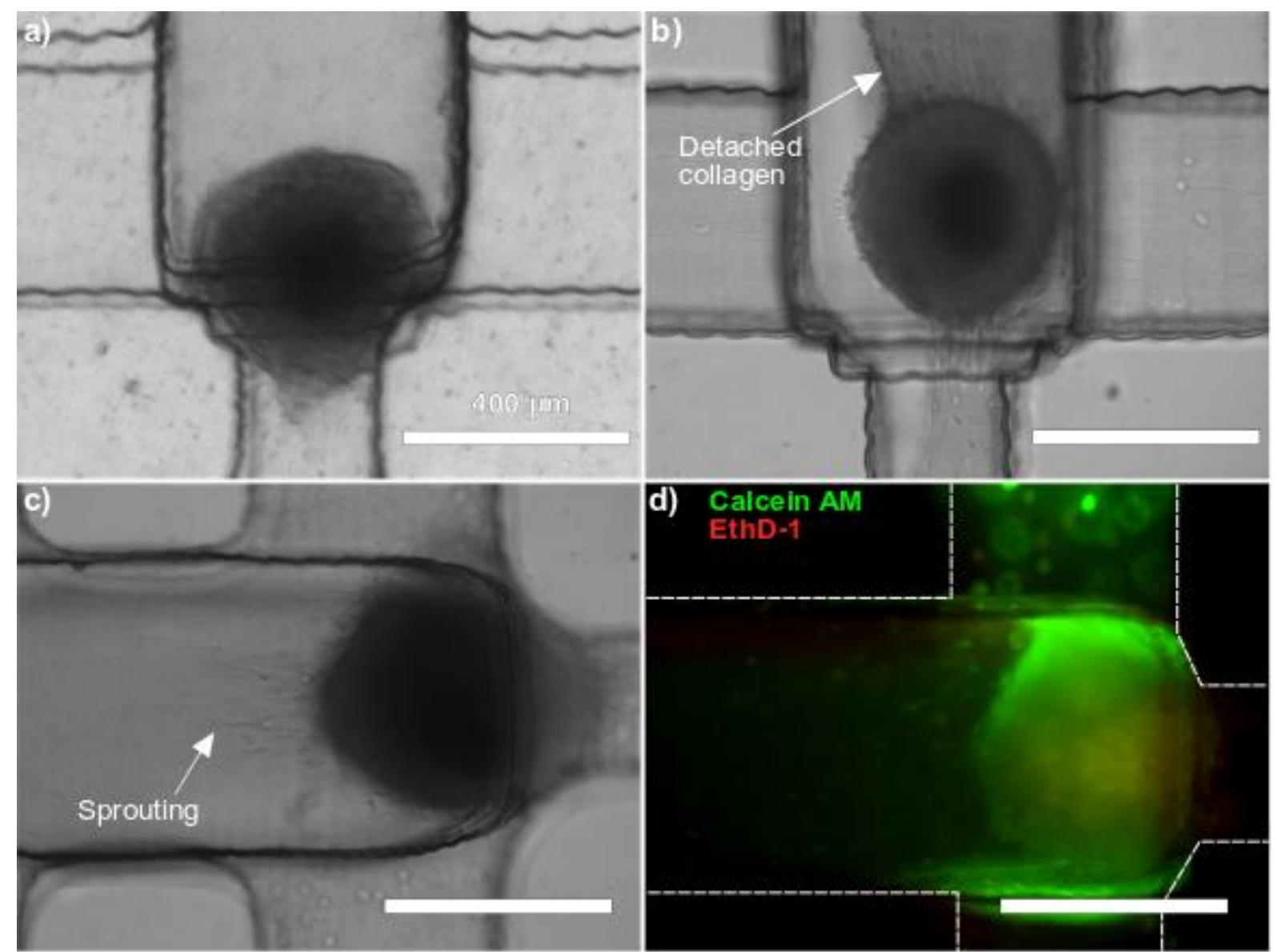

Figure 6-9 a) Spheroid in collagen I in the microfluidic device middle channel, after 30 min. reticulation of the collagen. b) Spheroid injected at day 4, after 24 h incubation. c) Spheroid injected at day 0, after $48 \mathrm{~h}$ incubation. d) Viability assay of the spheroid after $48 \mathrm{~h}$ incubation inside the microfluidic device. 
In a second experiment, the spheroid was injected in the constriction in the central channel, in collagen I, just after the lumen formation, but before seeding of the HUVECs cells. Here, no medium was injected in the central channel prior to adding the spheroid in collagen. After 48 $\mathrm{h}$ incubation, there was no indication of collagen hydrogel detaching from the walls (Figure 69.c). Additionally, cells were found to sprout out of the spheroid, mainly in the central channel $(c a .180 \mu \mathrm{m})$. Because of the slow progression of sprouting from the spheroid in the connecting channels, it was estimated that the neovascularization could be achieved, to connect the two large blood vessels, before the tumour spheroid could spread and occupy the connecting channels.

A viability assay was finally performed on the spheroid inside the microfluidic device, 48 $\mathrm{h}$ after its injection, indicating that it remained alive through the experiment (Figure 6-8.d). Only the side of the spheroid facing the constriction appeared dead, most likely due to the lack of access to nutrients.

\section{Conclusion}

In this chapter, we have developed a microfluidic platform in which two separate parallel blood vessels were generated inside a collagen lumen, and maintained alive for up to 7 days. We showed that these blood vessels possessed a tight endothelial barrier, with tight adherens junctions, and that they were leak-proof. We were able to perfuse our blood vessels without causing damage, and generating stretching of the endothelial cells.

By perfusing VEGF in the central channel, we successfully triggered the sprouting and migration of endothelial cells, from the large blood vessels toward the central channel, with indication of cell-cell interaction necessary for the formation of the neovasculature. In future work, we aim at characterizing these newly formed vessels, by looking at their stability over time, their openness, and their leakiness. If necessary, the VEGF concentration could be adjusted and additional growth factors included.

We were able to trap a single tumour spheroid in the device, aligned with the connecting channel, potentially able to connect it with the newly formed vessels. In future work, we aim at achieving the connection between the endothelial cells present in the connecting channels, 
and the ones sprouting from the tumour spheroid, bridging the two large blood vessels, and potentially allowing for perfusion through the capillary network. By controlling the pressure difference between the side channels, it will be then possible to control the flow-rate in the neovasculature, and observe the uptake of medicine in the tumour area.

\section{REFERENCES}

1. Carmeliet, P. and R.K. Jain, Angiogenesis in cancer and other diseases. Nature, 2000. 407(6801): p. 249-57.

2. Hillen, F. and A.W. Griffioen, Tumour vascularization: sprouting angiogenesis and beyond. Cancer Metastasis Rev, 2007. 26(3-4): p. 489-502.

3. Weiswald, L.B., D. Bellet, and V. Dangles-Marie, Spherical cancer models in tumor biology. Neoplasia, 2015. 17(1): p. 1-15.

4. Sant, S. and P.A. Johnston, The production of 3D tumor spheroids for cancer drug discovery. Drug Discov Today Technol, 2017. 23: p. 27-36.

5. $\quad$ Katt, M.E., et al., In Vitro Tumor Models: Advantages, Disadvantages, Variables, and Selecting the Right Platform. Front Bioeng Biotechnol, 2016. 4: p. 12.

6. Huang, Z. and S.D. Bao, Roles of main pro- and anti-angiogenic factors in tumor angiogenesis. World J Gastroenterol, 2004. 10(4): p. 463-70.

7. Conway, E.M., D. Collen, and P. Carmeliet, Molecular mechanisms of blood vessel growth. Cardiovascular Research, 2001. 49(3): p. 507-521.

8. Bentley, K. and S. Chakravartula, The temporal basis of angiogenesis. Philos Trans R Soc Lond B Biol Sci, 2017. 372(1720).

9. Maiullari, F., et al., A multi-cellular 3D bioprinting approach for vascularized heart tissue engineering based on HUVECs and iPSC-derived cardiomyocytes. Sci Rep, 2018. 8(1): p. 13532.

10. Sasmal, P., et al., 3D bioprinting for modelling vasculature. Microphysiol Syst, 2018. 2.

11. Bischel, L.L., et al., Tubeless microfluidic angiogenesis assay with three-dimensional endothelial-lined microvessels. Biomaterials, 2013. 34(5): p. 1471-7.

12. Chrobak, K.M., D.R. Potter, and J. Tien, Formation of perfused, functional microvascular tubes in vitro. Microvascular Research, 2006. 71(3): p. 185-196.

13. Bischel, L.L., S.H. Lee, and D.J. Beebe, A practical method for patterning lumens through ECM hydrogels via viscous finger patterning. J Lab Autom, 2012. 17(2): p. 96-103.

14. Walker, G. and D.J. Beebe, A passive pumping method for microfluidic devices. Lab Chip, 2002. 2(3): p. 131-4.

15. Kim, C., et al., A quantitative microfluidic angiogenesis screen for studying antiangiogenic therapeutic drugs. Lab Chip, 2015. 15(1): p. 301-10.

16. Jeong, G.S., et al., Sprouting angiogenesis under a chemical gradient regulated by interactions with an endothelial monolayer in a microfluidic platform. Anal Chem, 2011. 83(22): p. 8454-9. 
17. Yancopoulos, G.D., et al., Vascular-specific growth factors and blood vessel formation. Nature, 2000. 407(6801): p. 242-8.

18. Kobus, K., et al., Angiopoetin-2 (Ang-2) and Vascular Endothelial Growth Factor (Vegf) Play a Pivotal Role in the Development of Collaterals in Liver Cirrhosis in Humans. Hepatology, 2010. 52(4): p. 1069a-1069a.

19. Orimo, A., et al., Stromal fibroblasts present in invasive human breast carcinomas promote tumor growth and angiogenesis through elevated SDF-1/CXCL12 secretion. Cell, 2005. 121(3): p. 335-348.

20. Lamalice, L., F. Le Boeuf, and J. Huot, Endothelial cell migration during angiogenesis. Circulation Research, 2007. 100(6): p. 782-794.

21. Oh, S., et al., "Open-top" microfluidic device for in vitro three-dimensional capillary beds. Lab Chip, 2017. 17(20): p. 3405-3414.

22. Sobrino, A., et al., 3D microtumors in vitro supported by perfused vascular networks. Scientific Reports, 2016. 6.

23. Sano, E., et al., Engineering of vascularized 3D cell constructs to model cellular interactions through a vascular network. Biomicrofluidics, 2018. 12(4).

24. Shin, Y., et al., In vitro 3D collective sprouting angiogenesis under orchestrated ANG1 and VEGF gradients. Lab Chip, 2011. 11(13): p. 2175-81.

25. Chevalier, T., et al., Darcy's law for yield stress fluid flowing through a porous medium. Journal of Non-Newtonian Fluid Mechanics, 2013. 195: p. 57-66.

26. Ramanujan, S., et al., Diffusion and convection in collagen gels: Implications for transport in the tumor interstitium. Biophysical Journal, 2002. 83(3): p. 1650-1660.

27. Balcioglu, H.E., B. van de Water, and E.H. Danen, Tumor-induced remote ECM network orientation steers angiogenesis. Sci Rep, 2016. 6: p. 22580.

28. Blanco, R. and H. Gerhardt, VEGF and Notch in tip and stalk cell selection. Cold Spring Harb Perspect Med, 2013. 3(1): p. a006569.

29. Hicklin, D.J. and L.M. Ellis, Role of the vascular endothelial growth factor pathway in tumor growth and angiogenesis. J Clin Oncol, 2005. 23(5): p. 1011-27.

30. Minko, T., et al., The influence of cytotoxicity of macromolecules and of VEGF gene modulated vascular permeability on the enhanced permeability and retention effect in resistant solid tumors. Pharmaceutical Research, 2000. 17(5): p. 505-514.

31. Ridge, S.M., F.J. Sullivan, and S.A. Glynn, Mesenchymal stem cells: key players in cancer progression. Molecular Cancer, 2017. 16.

32. de Souza, L.E.B., et al., Mesenchymal Stem Cells and Pericytes: To What Extent Are They Related? Stem Cells and Development, 2016. 25(24): p. 1843-1852.

33. Spaeth, E.L., et al., Mesenchymal Stem Cell Transition to Tumor-Associated Fibroblasts Contributes to Fibrovascular Network Expansion and Tumor Progression. Plos One, 2009. 4(4).

34. Jeon, J.S., et al., Generation of $3 D$ functional microvascular networks with human mesenchymal stem cells in microfluidic systems. Integr Biol (Camb), 2014. 6(5): p. 55563.

35. Papaioannou, T.G. and C. Stefanadis, Vascular wall shear stress: basic principles and methods. Hellenic J Cardiol, 2005. 46(1): p. 9-15.

36. Li, Y.S.J., J.H. Haga, and S. Chien, Molecular basis of the effects of shear stress on vascular endothelial cells. Journal of Biomechanics, 2005. 38(10): p. 1949-1971.

37. Potters, W.V., et al., Measuring Wall Shear Stress Using Velocity-Encoded MRI. Current Cardiovascular Imaging Reports, 2014. 7(4).

38. Chappell, J.C., D.M. Wiley, and V.L. Bautch, Regulation of blood vessel sprouting. Semin Cell Dev Biol, 2011. 22(9): p. 1005-11. 
39. Wu, L., et al., Fibroblast growth factor-1 is a mesenchymal stromal cell-secreted factor stimulating proliferation of osteoarthritic chondrocytes in co-culture. Stem Cells Dev, 2013. 22(17): p. 2356-67.

\section{APPENDiX}

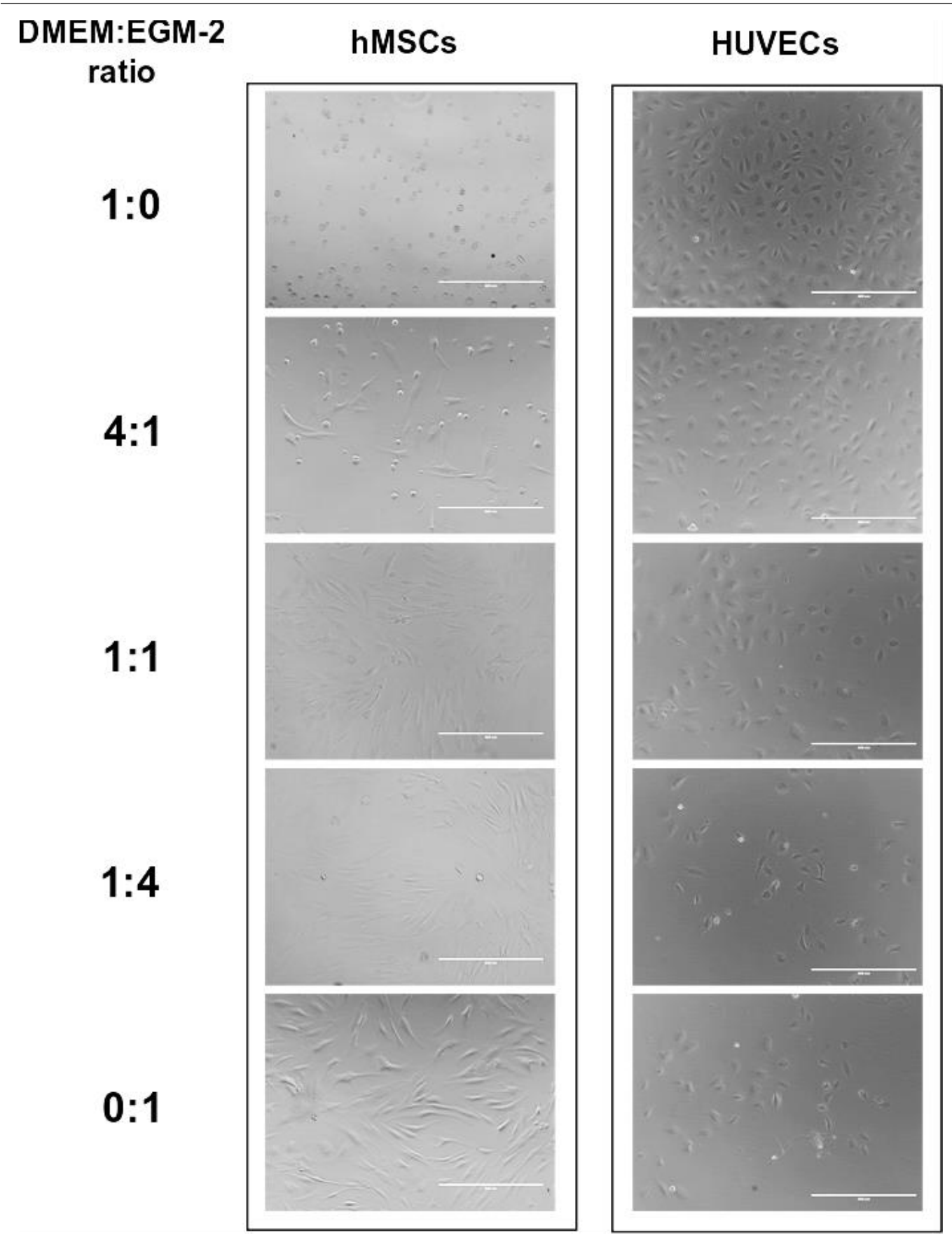

Figure 6-S1 Confluent monolayers of hMSCs and HUVECs, incubated in different ratios of DMEM:EGM-2 media for 48h. Both cell lines only survive in the 1:1 media ratio, which is then chosen for r experiments involving both cell types. 


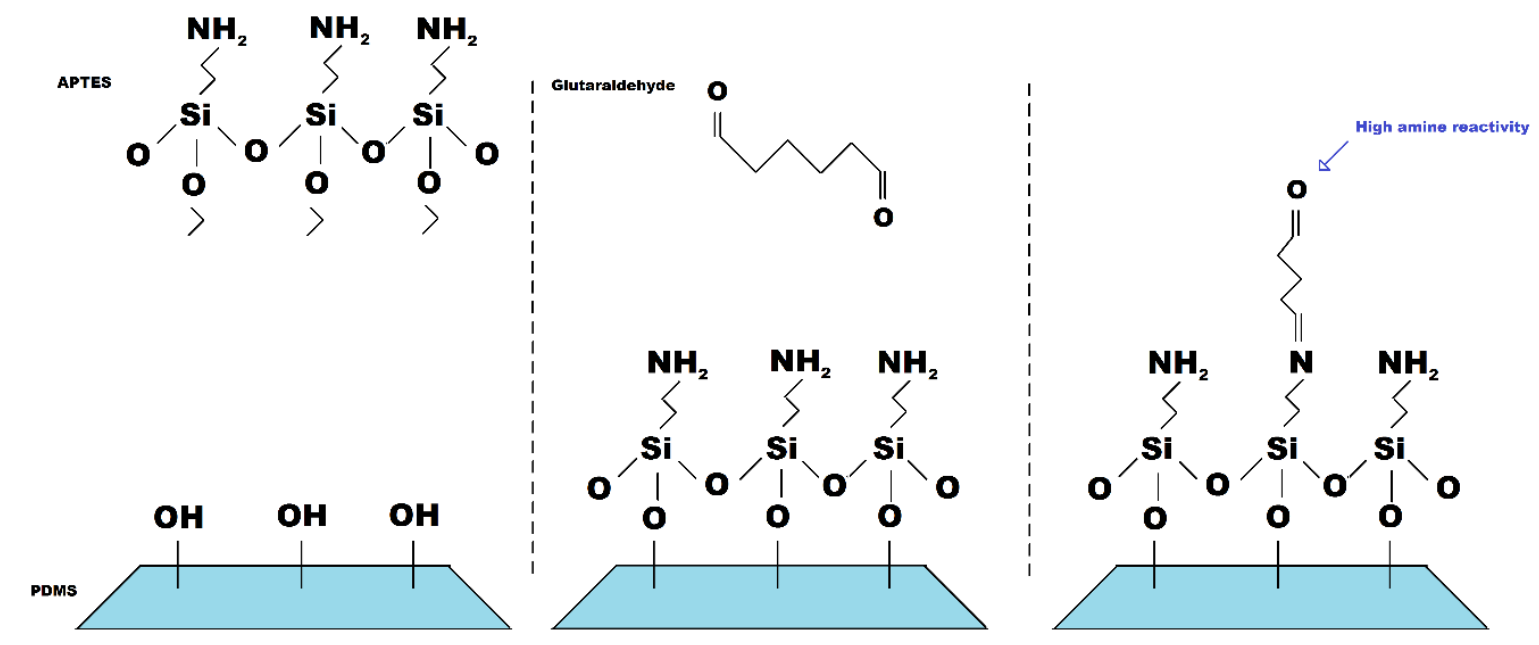

Figure 6-S2 APTES + glutaraldehyde coating of PDMS surface, creating a layer with high affinity to amine groups, as found in proteins present in the collagen hydrogel. 

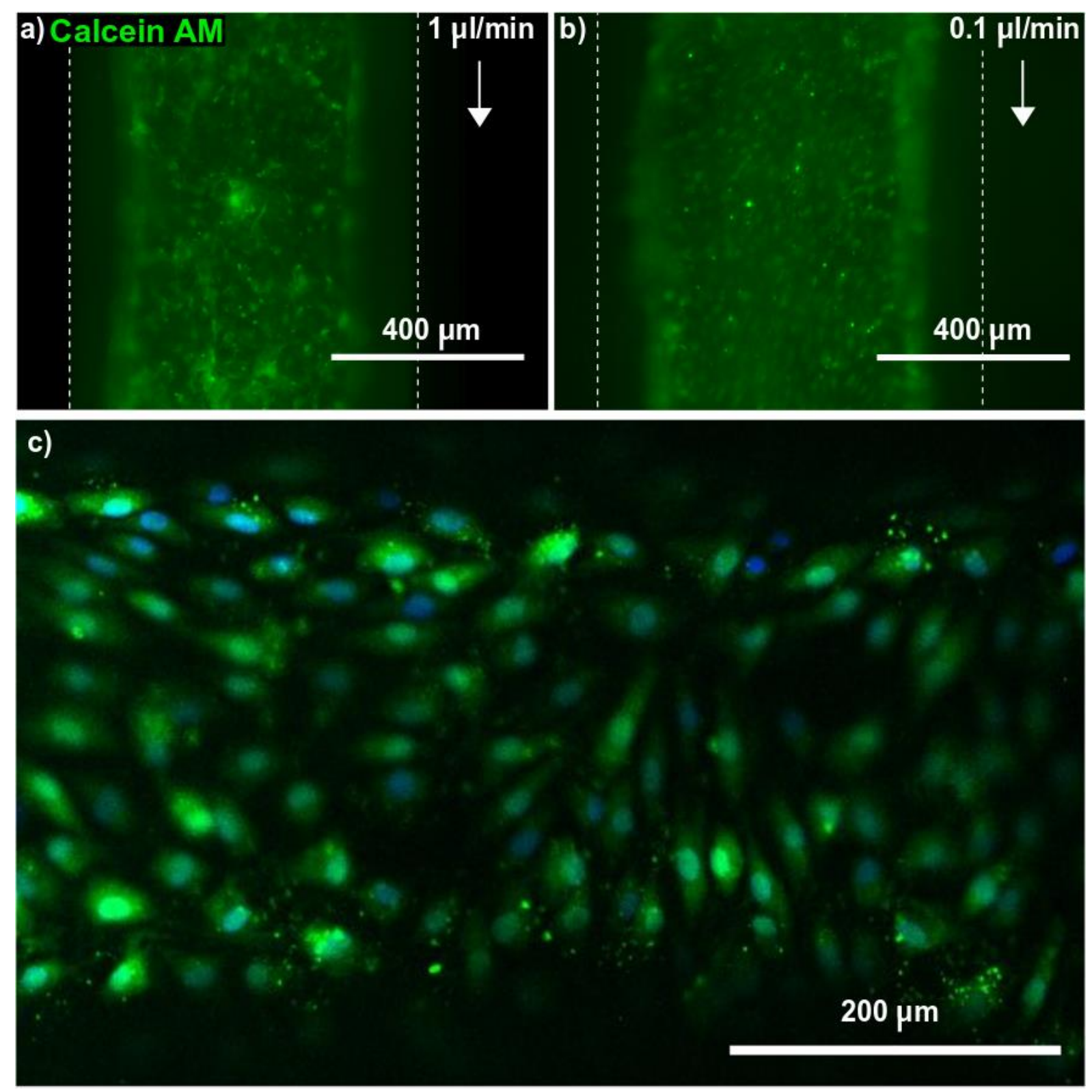

Figure 6-S3 Live assay (Calcein AM) of a large blood vessels, perfused for 24 h at (a) $1 \mu \mathrm{L} / \mathrm{min}$ or (b) $0.1 \mu \mathrm{L} / \mathrm{min}$. a) Portions of the blood vessels are missing, resulting in a compromised barrier. b) The endothelial barrier is preserved, and the cells show sign of rearrangment in the direction of the flow. $c$ ) Confocal imaging of the large blood vessel walls, with HUVEC cells fixed and stained with F-Actin. The cells show sign of conformation change, stretching into elongated cells, but are not aligned with the direction of the lumen. 


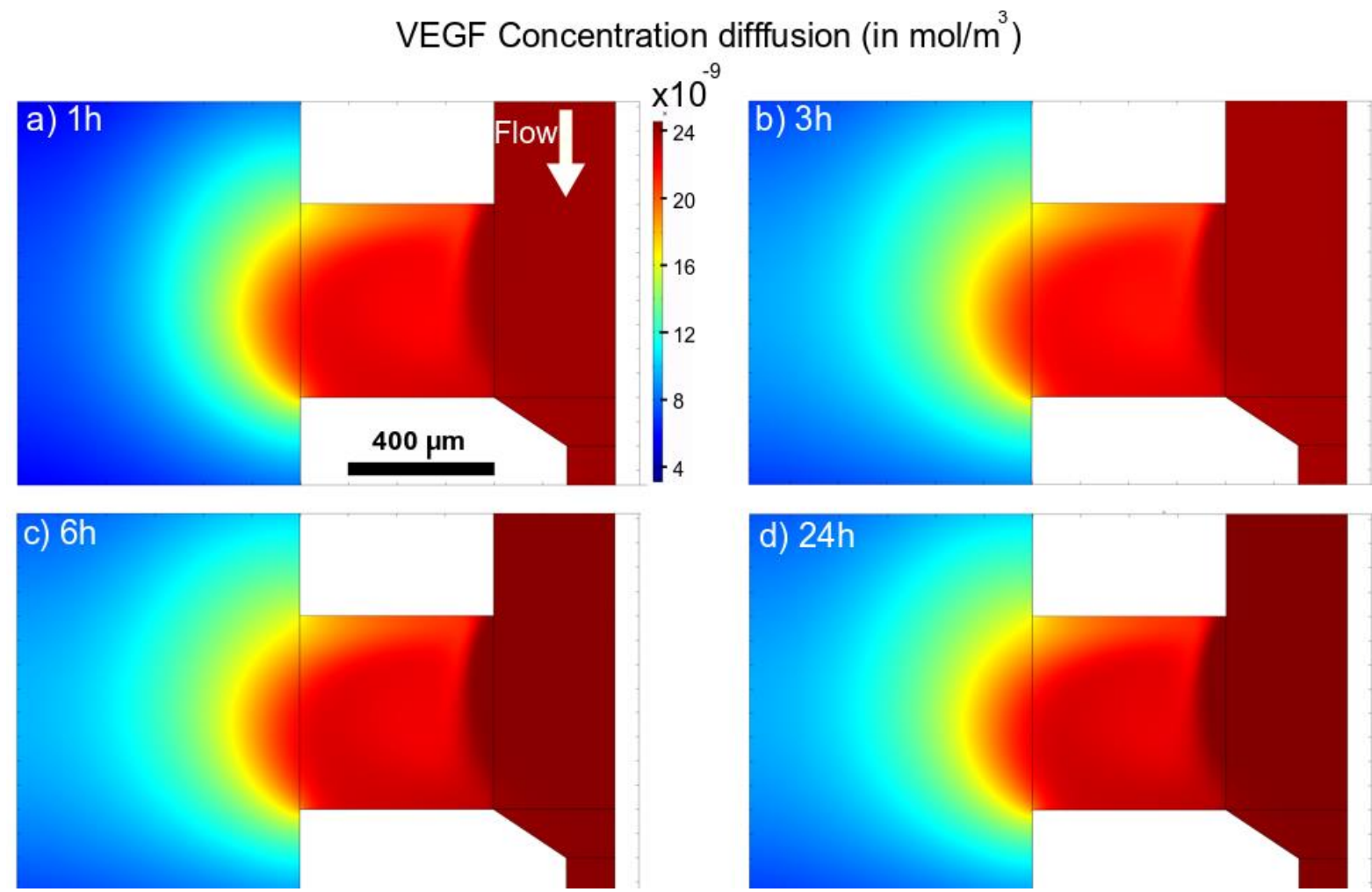

Figure 6-S4 COMSOL simulation of VEGF perfusion in central channel, diffusing in the lateral channel, after $1 \mathrm{~h}, 3 \mathrm{~h}, 6 \mathrm{~h}, 24 \mathrm{~h}$. After $24 \mathrm{~h}$ the gradient has slightly varied, but is still strongly present in the connecting channel. 
Chapter 7 - Summary and Outlook 


\section{Summary}

Tumour progression is a complex process and, despite originating from cancer cells, multiple cell types and other elements in the body promote its evolution. In this thesis, we took a look at some of these other elements involved in the tumour proliferation, namely the fibroblasts, known to secrete Extra Cellular Matrix (ECM) and the tumour-induced neovascularization, which provides the oxygen and nutrients required. We focused our attention on developing in vitro models using microfluidic systems to help testing new therapeutic treatments.

To achieve these goals, we first took a look at the most common tumour 3D in vitro model, the multicellular tumour spheroid (MCTS), and investigated how the MCTS protein expression profile compared with the in vivo condition (Chapter 2). We focused our attention on comparing the MCTS with the established 2D in vitro models, the monolayers. We also looked at how the size of MCTS could affect this protein expression profile. When performing a proteomic analysis of our murine cancer only spheroids, we observed that several metabolic pathways known to be promoted in tumours in vivo were also found up-regulated in the 3D model, as compared to the monolayer. Moreover, varying the size of the model greatly affected this protein expression, suggesting a variation-sensitive evolution inside the MCTS, most likely linked to its requirements (e.g., nutrients, growth factors) to further its proliferation.

In a second and narrower step, we looked at a few selected proteins expressed in the tumour and characterized their position inside the spheroid. However, since traditional tissue sectioning techniques are known to damage the tissues, and MCTS have shown lower structure integrity than ex vivo tissues, we decided to perform the analysis on the complete spheroids. To successfully perform high-resolution imaging on a 3D sample, we had to circumvent the lipidic cell membrane - induced light scattering resulting in dark images, by clearing the tissue using two different approaches. The first one follows the CLARITY (Clear Lipid-exchanged Anatomically Rigid Imaging/ immunostaining-compatible Tissue hYdrogel) and PACT (Passive CLARITY Technique) protocols (Chapter 3), consisting in fixing the proteins present inside the tumour spheroid to a polymer hydrogel, and using an organic solvent to remove the lipids. Using microfluidics to accelerate the process and reduce chemical requirements, we successfully cleared the tissue in a short amount of time. However, the solvent selected affected the proteins conformation, making it impossible to identify them using immunostaining techniques. In a second approach, we use the VISIKOL method, still consisting in removing 
the lipids, but without creating a polymer and using a softer detergent (Chapter 4). Using this method, we successfully cleared and immunostained the MCTS, and applied it to identify the distribution of oxygen and nutrients inside the tumour, as well as the impact of fibroblasts in the MCTS on the ECM production.

Next, to observe the impact of the fibroblasts and the resulting ECM on nanomedicine uptake, we designed a microfluidic device able to contain multiple MCTS (Chapter 5). In it, we investigated the penetration of silica nanoparticles, as models of nanomedicine, in tumour spheroids with or without fibroblasts, at different perfusion rate to mimic the different shear stresses found in vivo. At an interstitial shear stress $(0.001-0.01 \mathrm{~Pa})$ level, the presence of fibroblasts in the spheroid brought little change in the penetration depth of the nanoparticles in the tissue, but when the shear stress increased to more a vascular like (1 - $5 \mathrm{~Pa}$ ) condition, the difference became significant, and the nanomedicine penetration depth in co-culture MCTS of cancer cells and fibroblasts was greatly reduced compared to cancer only spheroids, especially for smaller nanoparticles. We observed no significant variation in the penetration profile when replacing the silica nanoparticles with liposomes, established organic nanocarrier for chemotherapy.

Finally, in the objective of presenting a new and more relevant in vitro model, we developed a microfluidic device able to trap a single tumour spheroid, surrounded by blood vessels. Using Collagen I as a model for ECM, we generated two large blood vessels on both sides of the MCTS, and by perfusing a gradient of growth factors (i.e., Vascular Endothelial Growth Factor) starting from the tumour site, we triggered the angiogenesis process, by which a neovascularization started to occur from the large blood vessels. We were able to integrate all the elements in the microfluidic device and initiate the angiogenesis, but did not complete the neovascularization process at this point, which would connect the large blood vessels together, and allow for a controlled perfusion of the MCTS. 


\section{Outlook}

The work presented in this thesis only scratches the surface of the potential of $3 \mathrm{D}$ in vitro models, as well as the possibilities of microfluidics as platforms to engineer and support these models. Several of the projects initiated here are being further investigated to improve the model, and better mimic the complete tumour microenvironment behavior.

First, in chapter 1, the proteomic study was limited to a single cellular composition presented, the cancer cells, whereas as we have described, multiple cell types play an active role in the tumour progression. Influence of fibroblasts in the tumour protein expression profile is currently being performed, and will be compared with the in vivo experiments, and using the cancer only spheroids as a reference. Later on, further experiments could be considered to integrate more cell types in the model, such as macrophages or adipocytes, also found highly present in breast cancer tissues.

We successfully performed in situ the clearing and immunostaining of MCTS, but only limited the investigation to a proof of concept by investigating phenomena we expected to observe in our spheroids (i.e., ECM production, oxygen induced behavioral changes). Ideally, this method could be exploited to investigate the 3D location of lesser known proteins. Such technique could be used for instance to locate proteins overexpressed in tumours, such as the one discussed in chapter 2, and identify whether these proteins could become targets for nanomedicine research.

Finally, as explained in this thesis, tissue vascularization is a key element of tissue engineering as well as organ-on-chip systems, and is being highly investigated. We successfully generated blood vessels and triggered angiogenesis in our system, but were unable to achieve successful vascularization of the tumour spheroid by the conclusion of the research contract. This research is currently being further pursued to improve the system and complete the tumour vascularization. Namely, the microfluidic system was redesigned to integrate more features to improve the survivability of the samples, and additional pro-angiogenic factors are being introduced to accelerate the angiogenesis and better stabilize the neovasculature.

This $\mathrm{PhD}$ project was originally proposed to research and develop a new nanomedicine models, integrating microbubbles as drug carriers, in the objective of testing these nanomedicines on $3 \mathrm{D}$ in vitro models. Through the unforeseeable challenges that occurred, as it does in any $\mathrm{PhD}$ 
research, it rapidly became apparent that, in the laboratory, the accuracy of the results obtained in drug development research was directly in correlation with the relevance of the in vitro models used. The project evolved therefore toward better understanding this model, and aiming at improving it, and in association improving the nanomedicine research field. Pharmaceutical companies are now also integrating microfluidic research facilities to develop 3D in vitro models like the MCTS for therapeutic assays, indicating that the progress made in the field of lab-on-chip and organ-on-chip systems has reached a level of interest justifying industrial development. By researching the tumour in vitro model during the last five years, I like to think that I participated to this progression, and that in a near future, microfluidics and organ-on-chip systems will become a major contributor to the research and development of new medicines. 


\section{Sammenvatting}

'Kanker' is de algemene naam die wordt gegeven aan een groep ziekten geassocieerd met abnormale celgroei en het ontstaan van tumoren. Deze tumoren kunnen de functie van het orgaan waarin ze zich bevinden ernstig verstoren. Kanker is één van de belangrijkste doodsoorzaken wereldwijd en is door zijn verschillende vormen en uiteenlopend ziekteverloop een bijzonder moeilijk te genezen ziekte. In de afgelopen decennia is een nieuwe klasse van veelbelovende complexe medicijnen ontwikkeld als alternatief geneesmiddel voor de bestaande chemotherapie. In deze zogeheten nanomedicijnen wordt een actief farmaceutische component gecombineerd met een nanodeeltje als drager. Met deze 'smart drugs' kan een hoge dosis medicatie zeer selectief worden toegediend aan het aangedane weefsel, terwijl tegelijkertijd het toxisch risico wordt verkleind.

Vanwege de complexiteit van nanomedicatie zijn traditionele 2D in vitro modellen niet langer afdoende om de potentie van deze nanomedicijnen volledig te kunnen onderzoeken. In dit proefschrift, getiteld "On-chip complex breast tumor microenvironment: Application to research in nanomedicine", worden alternatieve 3D in vitro modellen beschreven die een meer representatief beeld geven van de effectiviteit van de nieuw ontwikkelde nanomedicijnen. Dit promotieonderzoek maakt in eerste instantie gebruik van veelcellige tumor spheroids (MCTS) die enkel bestaan uit kankercellen, zoals in de meest voorkomende 3D-modellen. Met de MCTS wordt onderzocht hoe de grootte van deze spheroids de eiwitexpressie in de cel beïnvloedt. Daarna wordt de complexiteit van het model uitgebreid door toevoeging van fibroblasten aan de MCTS om vervolgens het effect van cellulaire hiërarchie, hypoxie en de afgifte van extracellulaire matrix te bestuderen. Hierbij wordt gebruikt gemaakt van verscheidene technieken om 3D-visualisatie van het weefsel mogelijk te maken. Vervolgens is er een tumor-on-chip platform ontwikkeld waarbij de MCTS modellen in een microfluïdisch compartiment geïsoleerd worden, zodat de afgifte van nanomedicijnen aan de tumor kan worden bestudeerd. De penetratie van de medicijnen in de MCTS is gekwantificeerd middels fluorescent gelabelde nanodeeltjes en hoge resolutie microscopie. Verscheidene parameters

zijn tijdens deze experimenten gevarieerd, waaronder de grootte van de nanodeeltjes, de vloeistofsnelheid en de samenstelling van de MCTS. Tot slot wordt er een platform gepresenteerd dat kan dienen als een micromilieu voor de tumor, waarin de MCTS wordt gecomplementeerd met bloedvaten waardoor nanomedicijnen intraveneus aan de tumor kunnen worden toegediend en hun effect kan worden geëvalueerd. 


\section{About the Author}

Jean-Baptiste Blondé was born on the $20^{\text {th }}$ of January 1990, in Rennes, France. After high school graduation in 2008, we started preparatory school in Rennes to prepare for the nation-wide entrance-competition to French engineering universities. In 2011, he was accepted in Télécom Physique Strasbourg, in TICsanté specialty (data and communication processing in health-related fields). He graduated in 2014, with a bachelor degree in biomedical engineering, completed

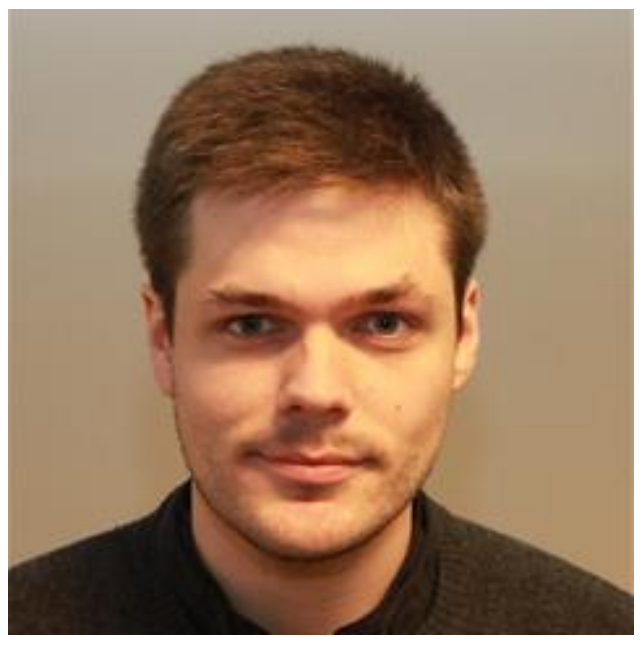
with a master degree in microelectronics.

Throughout his studies and internships, Jean-Baptiste discovered the technology of microfluidics, and its potential for a wide range of application. He first had the opportunity to work at Elvesys in Paris, where he helped develop a next generation of microscope compatible thermoregulator for better cell samples investigation, using microfluidics. Then, for his master final assignment, Jean-Baptiste joined the group of Prof. Piotr Garstecki at the Institute of Physical Chemistry, Warsaw, Poland. There, we worked on developing a fast method to assess and compare antibiotics efficacy on bacteria strains, using droplet microfluidics techniques.

After graduation, Jean-Baptiste started as a PhD student in Applied Microfluidics for BioEngineering Research group under the supervision of Prof. Dr. Ir. Séverine Le Gac at the University of Twente. His original project, MINACO, aimed at using monodisperse microbubbles as nanomedicine carrier to improve delivery and penetration of the drugs on in vitro tumor targets, using microfluidics. However, through a combination of challenges and opportunities, the project gradually deviated toward the improvement of the tumor model originally used as a target. Through this 5-year project, Jean-Baptiste tackled different aspect of the in vitro tumor model, from a cellular biology aspect, to a tissue engineering aspect. JeanBaptiste will defend his PhD-thesis on September $18^{\text {th }} 2020$ in Enschede, The Netherlands. 


\section{Scientific output}

Peer-reviewed journal article

Priwitaningrum D, Blondé J, Sridhar A, Baarlen J, Hennink W, Storm G, Le Gac S, Prakash J, Tumor stroma-containing 3D spheroid arrays: A tool to study nanoparticle penetration. $\mathrm{J}$ Control Release 244 (2016) 257-268.

\section{Conference contributions}

Blondé J, Babu D, Priwitaningrum D, Sridhar A, Prakash J, Le Gac S, MicroTAS 2016 (Poster Presentation), Studying nanoparticle-3D tumour spheroid interactions in a microfluidic format: Towards the assessment of drug delivery to complex tumour tissues under physiological conditions

Blondé J, Priwitaningrum D, Prakash J, Le Gac S, MicroTAS 2018 (Poster presentation), Tumour-on-a-chip platform to evaluate nanoparticles penetration in $3 \mathrm{D}$ co-culture tumour spheroids, November 11-15, 2018, Kaohsiung, Taiwan

Blondé J, Priwitaningrum D, Prakash J, Le Gac S, NanoBioTech-Montreux (Poster presentation), Studying Nanoparticle Penetration in 3D Multicellular Tumour Models OnChip, October 29-31, 2018, Montreux, Switzerland

Bokkers M, Blondé J, Ine Lentacker I, Michel Versluis M, Guillaume Lajoinie G, Le Gac S, Microtas conference 2018 (Poster presentation), Enhancing nanomedicine penetration in tumor-on-a-chip models using ultrasound-mediated microbubble activation, Kaohsiung (Taiwan), 11-15 Nov. 2018.

Asshoff S, Lancia F, Sukas S, Blondé J, Yamaguchi T, Hommersom C, Le Gac S, Katsonis N, MicroTAS 2015 (Poster Presentation), Photo-switchable distributors of light based on selfassembled monodisperse chiral nematic microspheres, October 25-29, 2015, Gyeongju, Korea 
Life Sciences Poster Award from the NanoBioTech Montreux conference, on "Studying Nanoparticle Penetration in 3D Multicellular Tumour Models On-Chip”, October 31, 2018, Montreux, Switzerland 


\section{Acknowledgment}

I would like to take the time to thank all the people who made this $\mathrm{PhD}$ and this thesis possible.

First and foremost, I want to thank my promotor and daily supervisor Prof. dr. ir. Séverine Le Gac, for offering me the $\mathrm{PhD}$ position, and for advising me during the five-and-a-half years of my $\mathrm{PhD}$. Despite the project having its ups and downs, and us not always seeing eye-to-eye on the paths to choose, I really appreciated the knowledge you shared with me, and the freedom of action you gave me. I really enjoyed the work we did together, and I think that together we managed to try a lot of different and interesting work.

I then want to thank, in the order I met them, my colleagues and friends at AMBER whom I worked alongside with. I first have to thank Adithya, who was here when I started, who showed me the ropes, and who was and still is a good friend to me. You made the office a fun place to be, especially on Friday afternoons. I then want to thank Renée, who started roughly at the same time as me, and has been a good officemate and confident throughout my $\mathrm{PhD}$. You have taught me the importance of a proper balance between work and everything else. I also want to thank Yawar, you are a good friend and a good traveling companion. I then want to thank Bastien, who ensured the dominion of the French in the group. I really enjoyed your friendship, but also your expertise, which lead to numerous engineering and scientific debates these last three years. Finally, I thank Carlo Alberto, who came in our office smashing through the door. You may have been overwhelming at first, but you have brought a welcoming breath of fresh air to our group.

I also want to thank my other colleagues whom I have shared the hallways and the labs with. I will not cite all of them here, but I would especially like to thank Jan, Tom, Lisanne, and Michelle at DBE, Yusuf, Rolf, Aisen, Marcello, and Simone, at AST, and Fabian and Jiena at BME. All of you made the University of Twente an interesting and exciting place to be.

I of course do not forget the crab people, who among other things have been my daily lunch partners for almost five years. I am especially talking about Josh, Hai, Miquel, Marzena, Vasilis, Jeroen, Christina and Corentin but they are not the only one.

I would especially like to thank Kasya, who in the last few years has become a very close friend. You have always been there for the good and for the bad, and I don't think I could have arrived where I am today if it wasn't for you. 
I do not forget my students, first the masters Barbara and Ana, who's motivation and dedication have been of great help to me. I also thank my ten bachelor students, William, Martijn, Stanislas, Danya, Marieke, Janine, Jerilaine, Niels, Sandy and Dzeneta for the work they did. You helped reduce my workload and made my life a little easier.

I would like to thank the people I have worked with, starting the partners and collaborators. I would especially like to thank Prof. Dr. Jai Prakash for his contribution and guidance in the first part of my PhD. I thank Dwi for her collaboration and her help in the tumor spheroid project. I also would like to thank Guillaume on his collaboration on the sonoporation project, and even though it failed, I received a lot of valuable input from you. I would like to thank our collaborators in Lille Prof. Dr. Christian Rolando and Dr. Fabrice Bray, for their contribution to the proteomics project.

During my $\mathrm{PhD}$, I had difficulties staying put, and moved places four times. I would like to therefore to tell my former roommates Stefan, Hai, Corentin, Miquel, Yusuf and Carlo, that I had a great time sharing the daily life outside of work with you.

Finally, I would like to thank my family, especially my parents Jean and Françoise, who have been very supportive and patient with me through the stress and struggles. I know I am not the most communicative person, and I have caused you to worry, but this is now over. Don't worry, I will find something else to make you worry about. 